University of Rhode Island

DigitalCommons@URI

Open Access Dissertations

2019

\title{
OVERCOMING CONTEMPORARY OBSTACLES IN DRUG DELIVERY VIA ACETALATED DEXTRAN PARTICLE FORMULATIONS
}

Nishan K. Shah

University of Rhode Island, nishanshah0717@gmail.com

Follow this and additional works at: https://digitalcommons.uri.edu/oa_diss

\section{Recommended Citation}

Shah, Nishan K., "OVERCOMING CONTEMPORARY OBSTACLES IN DRUG DELIVERY VIA ACETALATED DEXTRAN PARTICLE FORMULATIONS" (2019). Open Access Dissertations. Paper 834.

https://digitalcommons.uri.edu/oa_diss/834

This Dissertation is brought to you for free and open access by DigitalCommons@URI. It has been accepted for inclusion in Open Access Dissertations by an authorized administrator of DigitalCommons@URI. For more information, please contact digitalcommons-group@uri.edu. 
OVERCOMING CONTEMPORARY OBSTACLES IN DRUG DELIVERY VIA ACETALATED DEXTRAN PARTICLE FORMULATIONS

BY

NISHAN KALPESH SHAH

A DISSERTATION SUBMITTED IN PARTIAL FULFILLMENT OF THE REQUIREMENTS FOR THE DEGREE OF

DOCTOR OF PHILOSOPHY

IN

PHARMACEUTICAL SCIENCES

UNIVERSITY OF RHODE ISLAND

2019 
DOCTOR OF PHILOSOPHY DISSERTATION

$\mathrm{OF}$

NISHAN KALPESH SHAH

\section{APPROVED:}

Dissertation Committee:

Major Professor Samantha A. Meenach

Jie Shen

Michael Greenfield

Nasser H. Zawia

DEAN OF THE GRADUATE SCHOOL

UNIVERSITY OF RHODE ISLAND 


\begin{abstract}
Particle-based delivery systems have been investigated for their potential to increase the efficacy of patient diagnoses and treatments. In many cases, these systems increase the bioavailability and/or decrease the toxicity of clinically relevant drugs. These developments have led to improvements in patient compliance, morbidity, mortality, and quality of life. To date, particle-based delivery systems have been evaluated in hundreds of clinical trials around the world. Unlike conventional delivery systems, particle-based delivery systems offer increased surface area, colloidal stability, and system tunability, all of which can be tailored to target the disease state of interest and specific patient population.
\end{abstract}

Although there are examples of successful particle-based drug products, there are numerous obstacles that consistently shunt the ability for these systems to be translated from benchside to bedside. Two consistent obstacles are drug toxicity and scale-up manufacturing. Drug toxicity is largely caused by inefficient targeting, inopportune routes of administration (i.e. oral delivery for a pulmonary disease), and unfavorable release mechanisms. Scale-up manufacturing is a continual industrial interest, given that particle-based drug products are increasing in popularity, however scale-up procedures are still in development and face their own challenges. A potential method to overcome the obstacles faced in drug delivery is the development of new polymers, either by synthesis of novel entities or derivatization of current ones. To ensure biocompatibility, the latter is a common practice. Biopolymers such as chitosan, hyaluronic acid, poly(lactic-co-glycolic acid) (PLGA), and naturally occurring polysaccharides undergo modifications to achieve desirable characteristics 
for drug delivery. Acetalated dextran (Ac-Dex) is a synthetic biopolymer derived from dextran, a naturally produced hydrophilic polysaccharide. Following a one-step reaction, the hydroxyl moieties of dextran are converted to acetal groups, transitioning the biopolymer from hydrophilic to lipophilic solubility. Hydroxyl moieties still present on the backbone can provide a handle for ligand attachment to actively target specific sites of the body. Additionally, controlling the reaction time and altering the molecular weight of the dextran backbone can alter the degradation kinetics, providing flexibility to achieve desirable release kinetics for a therapeutic of interest. Overall, Ac-Dex demonstrates cost-effective and efficient synthesis, easy tunability for targeting, and flexibility in controlling release kinetics, all of which propitiate its promise as a drug carrier.

The purpose of this dissertation was to utilize the advantages of Ac-Dex to investigate its potential as a drug carrier to overcome challenges that exist in the field of drug delivery. Manuscript 1 focuses on decreasing drug toxicity using active targeting. Here, Ac-Dex nanoparticles (NP) were synthesized and coated with phosphatidylserine to instigate macrophage uptake for the potential to treat diseases that use these cells as reservoirs, such as tuberculosis and HIV. Manuscript 2 focuses on the synthesis of Ac-Dex microparticles (MP), followed by an exploration of their ability to modulate the release of water-soluble cargo. Ac-Dex MP were synthesized via spray drying and were loaded with a water-soluble dye. Following the synthesis, MP were evaluated for their characteristics and drug release behaviors in multiple $\mathrm{pH}$ environments. Manuscript 3 uses Ac-Dex as an economical model drug carrier and focused on studying the effects of tangential flow filtration (TFF) parameters (factors) 
on the characteristics of NP (responses) to explore its promise as a scale-up purification technique. The final manuscript focuses on the physical characterization and development of nanocomposite microparticle system for localized delivery of the small molecule Eact, a potential therapeutic for pulmonary arterial hypertension.

Previously published reports show that Ac-Dex demonstrates significant promise as a versatile, cost-and time-effective, and promising drug carrier for a range of applications and disease states. The manuscripts in this dissertation all explore uncharted territories of Ac-Dex's potential, further demonstrating its versatility and promise to overcome current drug delivery obstacles. 


\section{ACKNOWLEDGMENTS}

From the bottom of my heart, I would like to thank my loving parents, Jeanette and Kalpesh Shah. The sacrifices that you both have made to provide for both Karishma and I is unmatched. Your continual love and support, even on the worst days, have helped me grow to become the man I am today. I love you both dearly.

To my sister Karishma, my brother-in-law Bobby, my nephew Aditya, and my niece Priyanka, thank you for the love and the laughs we have shared. I cannot imagine what my life would be like without you all. Karishma, you are my best friend and I am the luckiest brother. Bobby, you are the big brother I have always wanted and needed. Aditya and Priyanka, Uncle Nishan loves you both very much. You are both a continual inspiration to me and are a constant reminder of why I do what I do.

Thank you Dr. Meenach, for your guidance, support, and insight for both my graduate studies and my everyday life. I am so grateful for you allowing me to join your group halfway through my first year of school. Since then, I have learned how to become an independent thinker, scientist, and person. Thank you to my other committee members, Drs. Shen, Greenfield, and Roxbury for not only your contributions to my dissertation, but also to my growth as a scientist.

Thank you to my lab mates Sweta, Zimeng, Jakaria, Ryan, Elisa, and Andrew for your support, and your help with lab work and life obstacles. To my friends Adam, Chrissy, Riley, Meg, Ben, Shelby, Gina, Ken, Nick, Meiwen, Millea, and everyone else, thank you for being my friends and providing me with the support system I needed to get through the obstacles of my research and my life. You all have taught me that, just like life itself, graduate school is a team sport, and you cannot do it alone. 
As a graduate student, I was blessed with the opportunity of being a teaching assistant for 4 years, for both PharmD and BSPS students. To all the students that I interacted with and taught, thank you all for teaching me things about myself, and for making me a better and more confident teacher. I am blessed to have not only learned all your names and personalities, but also to have gotten a chance to see you all grow into scientists, scholars, and clinical professionals. I know that wherever you all go, you will do great things in your careers. A special thank you to Drs. Allababidi, Lu, Chen, and Morisseau for giving me the opportunity to teach alongside them.

Finally, I would like to thank my martial arts instructor, David Ahrens. I joined your school 18 years ago as a shy 9-year-old hiding behind the leg of his mom before his first class. Since then, with your guidance, support, love, and mentorship, you were able to help mold me into what I am today as a teacher, martial artist, scientist, critical thinker, and person. No matter where I will go, I know that I will always be part of the East Coast Karate family, and I will always have a home there. I will forever be grateful for what you and everyone else at East Coast Karate have done for me. 


\section{PREFACE}

This dissertation has been written in manuscript style format. Chapter 1 is an introduction to the dissertation. Chapter 2 entitled "Particle technology and routes of administration: parenteral and pulmonary delivery" is an in-depth introduction to both parenteral and pulmonary administration routes for particle technology, both of which are partially adopted from Chapter 6 of the book "Nanoparticles for Biomedical Applications." Chapter 3 entitled "Enhancement of Macrophage Uptake via Phosphatidylserine-Coated Acetalated Dextran Nanoparticles" was accepted to Journal of Drug Delivery Science and Technology in January 2019. Chapter 4, entitled "Sustained Release of Water-Soluble Therapeutics via Dry Powder Aerosol Acetalated Dextran Microparticles" was submitted to Drug Development and Industrial Pharmacy in April 2019. Chapter 5, entitled "Comparison of Nanoparticle Purification Process via Centrifugation and Tangential Flow Filtration: A Case Study of Acetalated Dextran Nanoparticles" was submitted to Particuology in April 2019. Chapter 6 entitled "Physical Characterization and Formulation Development of Eact for the Treatment of Pulmonary Arterial Hypertension" shows preliminary data pertaining to the project. Chapter 7 includes conclusions and future work related to this dissertation. 


\section{TABLE OF CONTENTS}

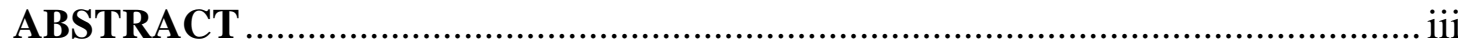

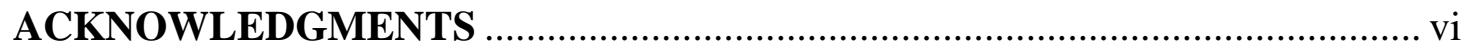

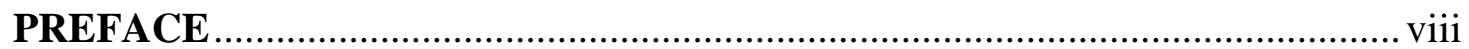

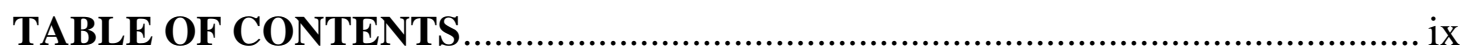

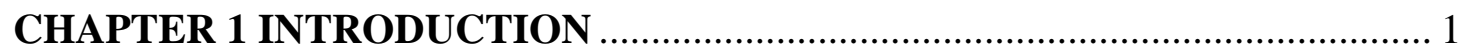

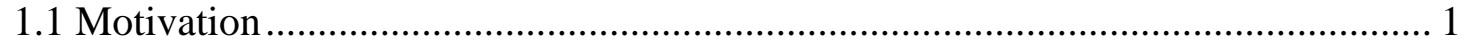

1.2 Significance of This Work ……………………............................................ 7

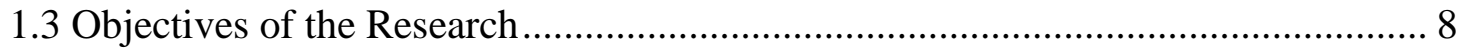

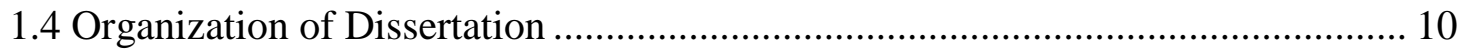

CHAPTER 2 PARTICLE TECHNOLOGY AND ROUTES OF ADMINISTRATION: PARENTERAL AND PULMONARY DELIVERY .. 12

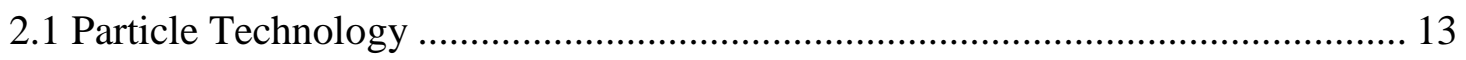

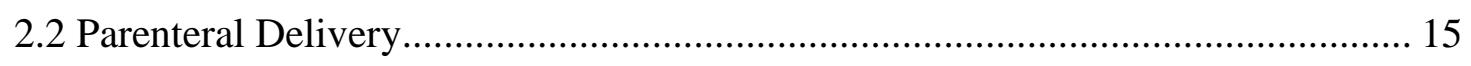

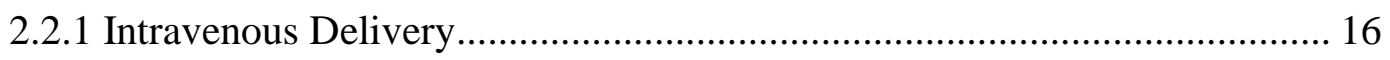

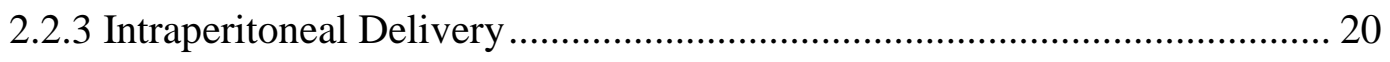

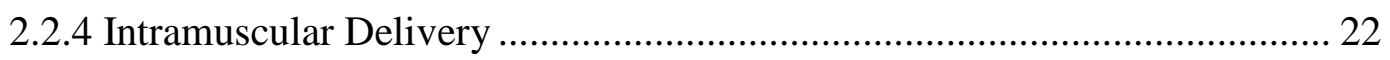

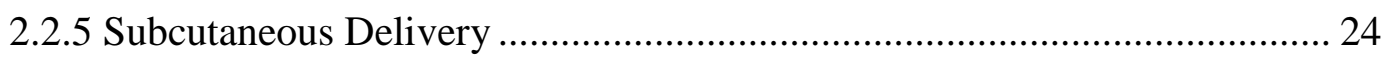

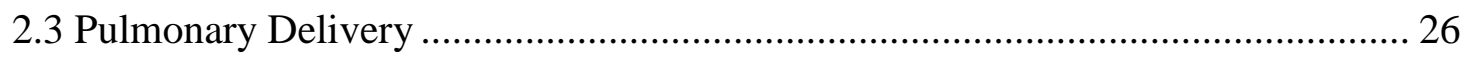

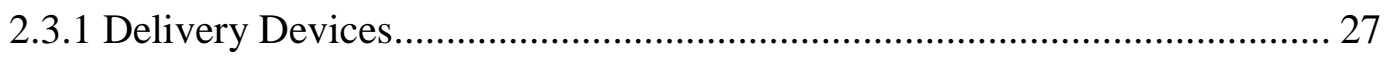

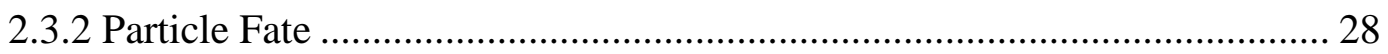

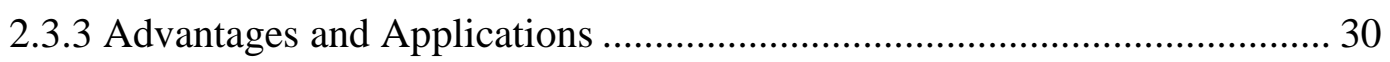

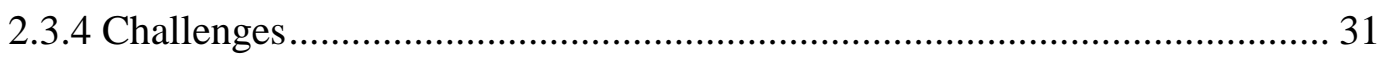

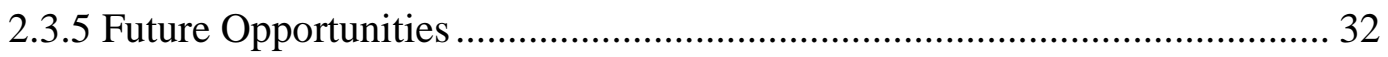

CHAPTER 3 ENHANCEMENT OF MACROPHAGE UPTAKE VIA PHOSPHATIDYLSERINE-COATED ACETALATED DEXTRAN

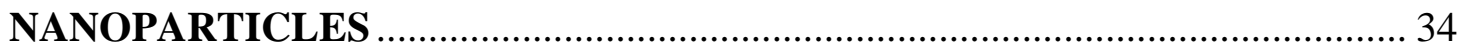

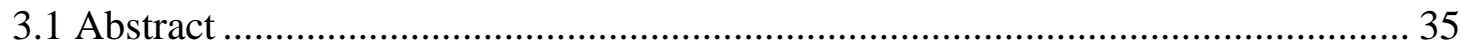

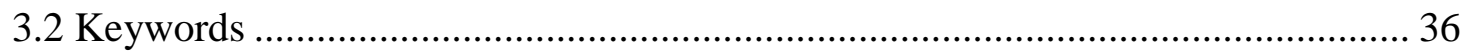

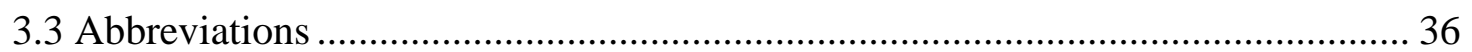

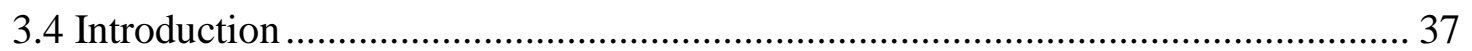

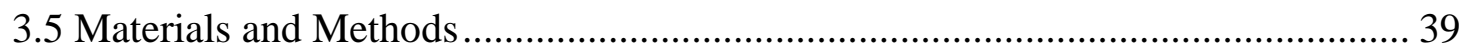

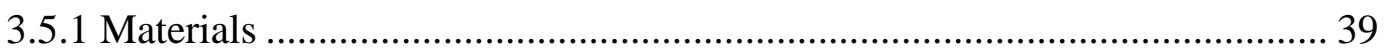

3.5.2 Synthesis and Characterization of Acetalated Dextran................................. 40

3.5.3 Synthesis of PVA-Coated Nanoparticles .................................................... 40

3.5.4 Synthesis of DPPS and DPPC-Coated Ac-Dex NP ...................................... 41

3.5.5 Nanoparticle Size and Surface Charge Characterization ............................... 41 
3.5.6 Evaluation of CUR NP Encapsulation Efficiency and Drug Loading......... 42

3.5.7 Electron Microscopy Imaging Analysis of Nanoparticles ........................... 42

3.5.8 Differential Scanning Calorimetry (DSC) Analysis of Nanoparticles......... 43

3.5.9 PVA Coating Quantification.................................................................. 43

3.5.10 Phospholipid Content Quantification.......................................................... 44

3.5.11 In Vitro Drug Release from CUR NP ................................................... 44

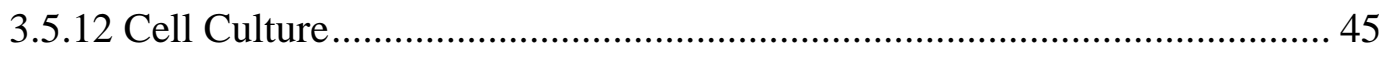

3.5.13 In Vitro Cytotoxicity Analysis of NP ..................................................... 45

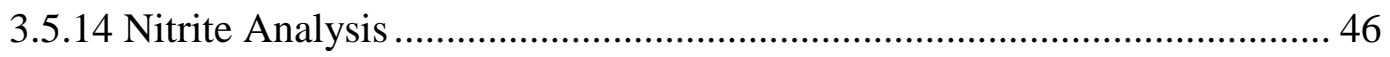

3.5.15 In Vitro Cellular Uptake Via Spectroscopy and Confocal Microscopy .... 46

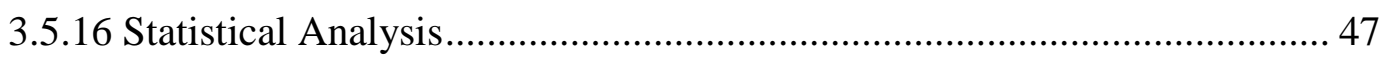

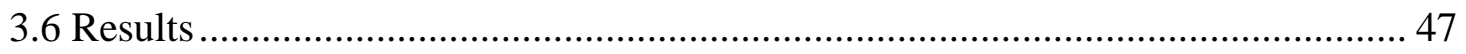

3.6.1 Characterization of Ac-Dex Polymer and Nanoparticles ............................ 47

3.6.2 Morphological Analysis of NP Via Electron Microscopy............................. 49

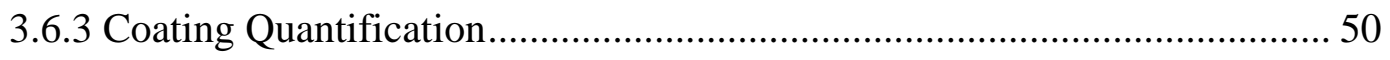

3.6.4 Differential Scanning Calorimetry (DSC) Thermal Analysis....................... 50

3.6.5 In Vitro Drug Release from NP ........................................................... 53

3.6.6 In Vitro Cytotoxicity Assay ...................................................................... 54

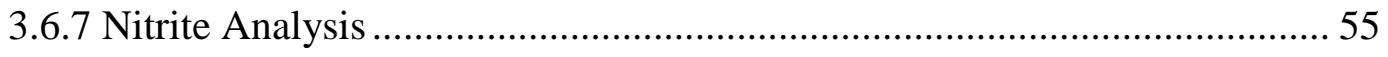

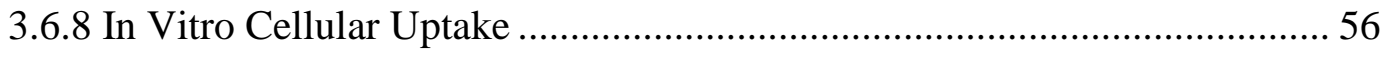

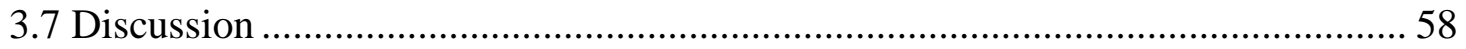

3.7.1 Nanoparticle Design and Analysis........................................................ 58

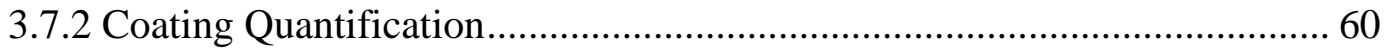

3.7.3 Thermal Analysis of Nanoparticle Formulations.......................................... 60

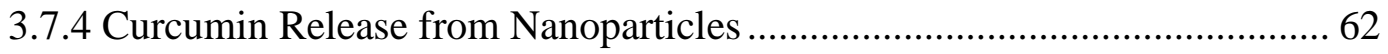

3.7.5 Cytotoxic Analysis and Macrophage Uptake of Nanoparticles .................... 62

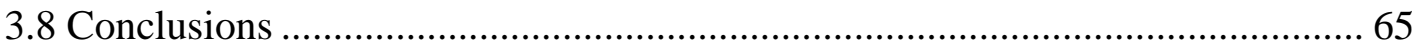

CHAPTER 4 SUSTAINED RELEASE OF WATER-SOLUBLE THERAPEUTICS VIA DRY POWDER AEROSOL ACETALATED

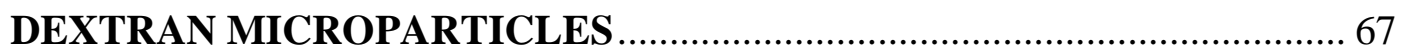

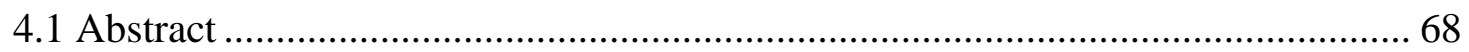

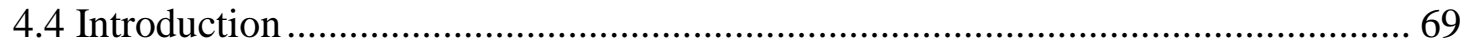

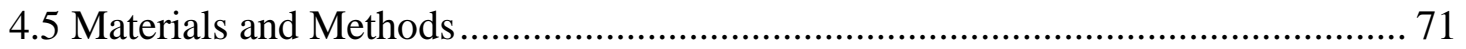

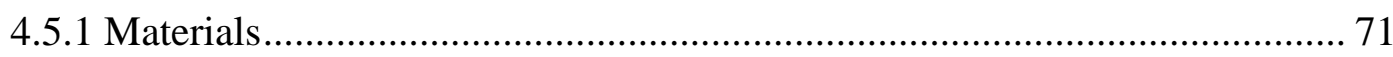

4.5.2 Synthesis and characterization of acetalated dextran.................................... 72

4.5.3 Microparticle synthesis via spray drying ...................................................... 72

4.5.4 Morphology, size, and tapped density analysis............................................. 74 


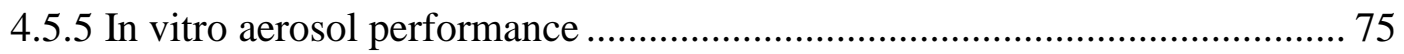

4.5.6 Crystallinity and phase transition analysis ....................................................... 76

4.5.7 Analysis of water content via Karl Fischer titration ..................................... 77

4.5.8 Encapsulation efficiency and drug loading analysis ...................................... 77

4.5.9 Drug release and release kinetic analysis..................................................... 77

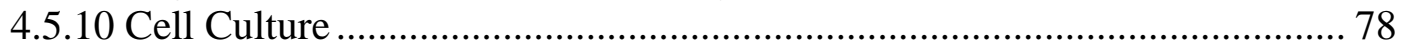

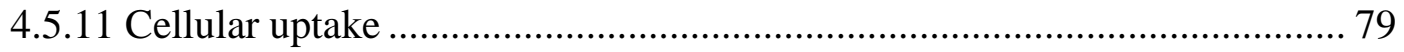

4.5.12 In vitro cytotoxicity assay ……………………...................................... 79

4.5.13 In vitro transepithelial electrical resistance of lung epithelial cells exposed

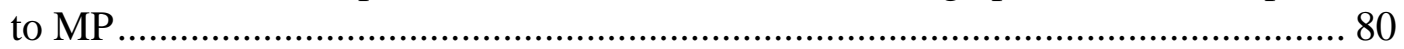

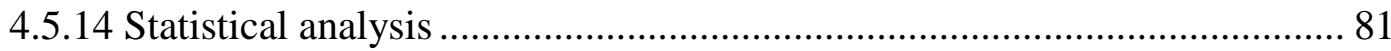

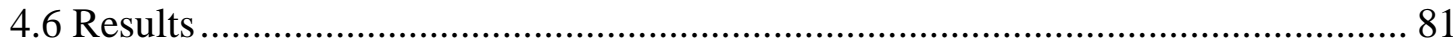

4.6.1 Confirmation of acetalated dextran (Ac-Dex) synthesis and microparticle

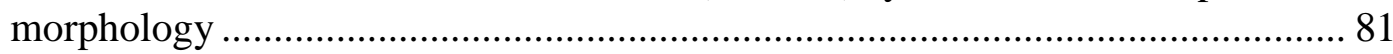

4.6.2 Size analysis and in vitro aerosol performance........................................ 82

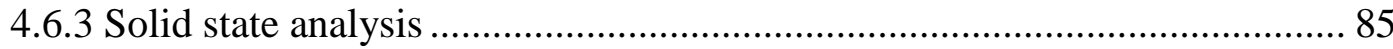

4.6.4 Drug loading, in vitro drug release, and release kinetic analysis.................. 87

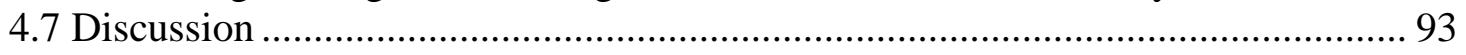

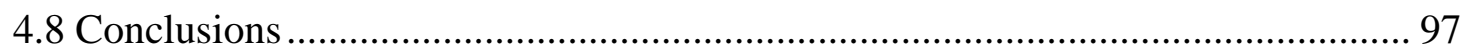

CHAPTER 5 COMPARISON OF NANOPARTICLE PURIFICATION PROCESS VIA CENTRIFUGATION AND TANGENTIAL FLOW FILTRATION: A CASE STUDY ON ACETALATED DEXTRA

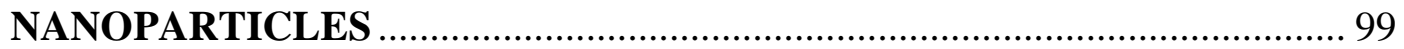

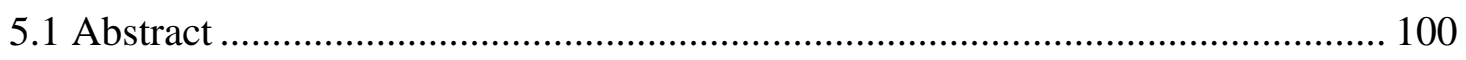

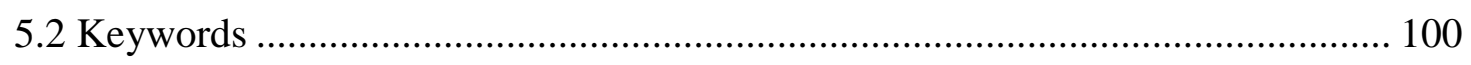

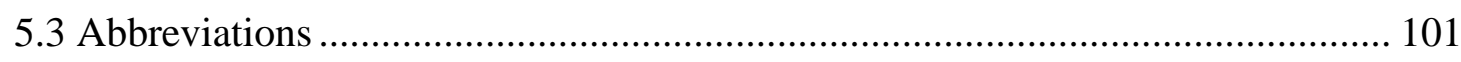

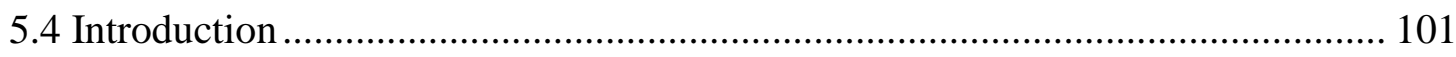

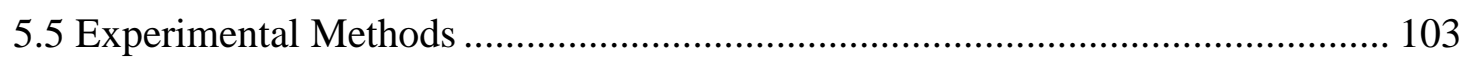

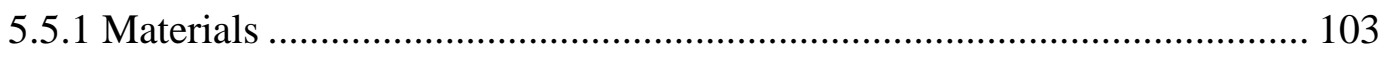

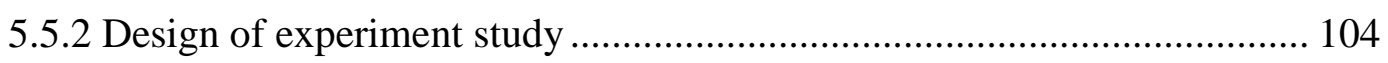

5.5.3 Comparison of the purification processes.................................................. 107

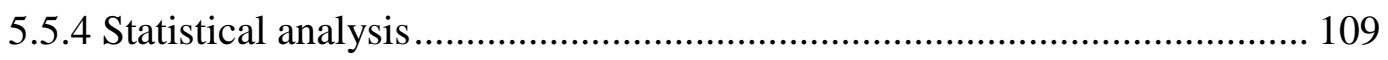

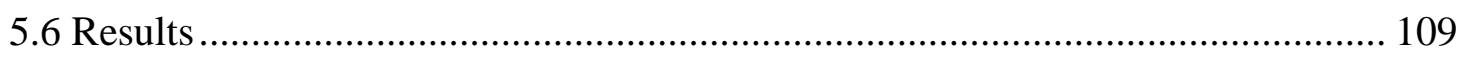

5.6.1 Design of experiment results for PTX-loaded Ac-Dex NP (PTX-NP)...... 109

5.6.2 Comparison of purification processes....................................................... 113

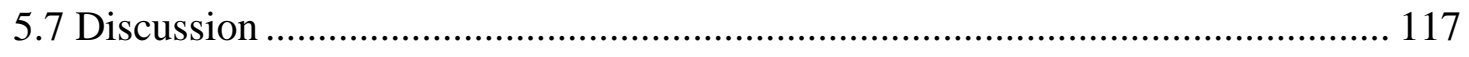

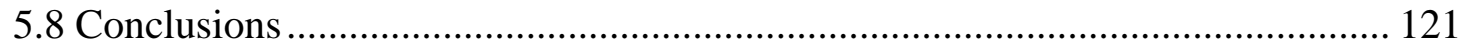

CHAPTER 6 PHYSICAL CHARACTERIZATION AND FORMULATION DEVELOPMENT OF Eact FOR THE TREATMENT OF PULMONARY ARTERIAL HYPERTENSION ……………………............................ 122

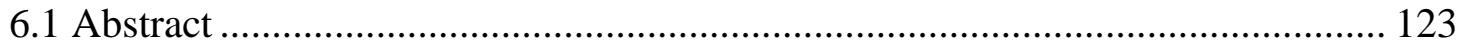




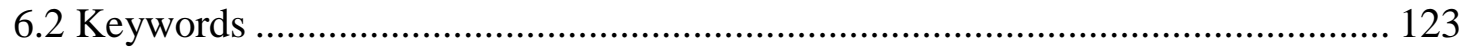

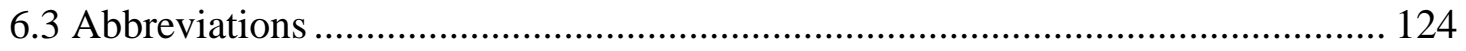

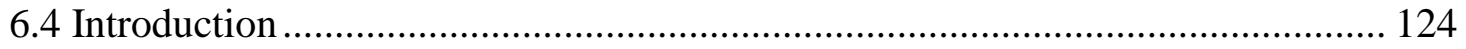

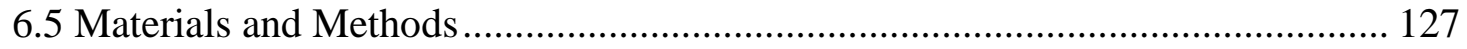

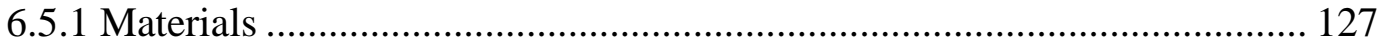

6.5.2 Synthesis and Characterization of Acetalated Dextran............................... 127

6.5.3 Synthesis of Eact-Encapsulated Nanoparticles........................................... 127

6.5.4 nCmP Synthesis Via Spray Drying...................................................... 128

6.5.5 Nanoparticle Size and Surface Charge Characterization ............................ 128

6.5.6 Particle Morphology and Shape Analysis via Scanning Electron Microscopy

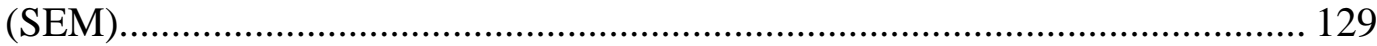

6.5.7 Crystallinity and Phase Transition Analysis .............................................. 129

6.5.8 Karl Fischer Coulometric Titration....................................................... 130

6.5.9 In Vitro Aerosol Performance................................................................ 130

6.5.10 Encapsulation Efficiency and Drug Loading Analysis............................. 132

6.5.11 Statistical Analysis............................................................................. 132

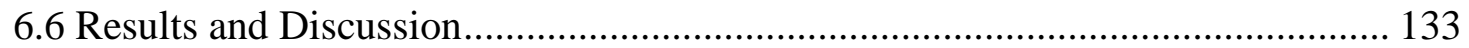

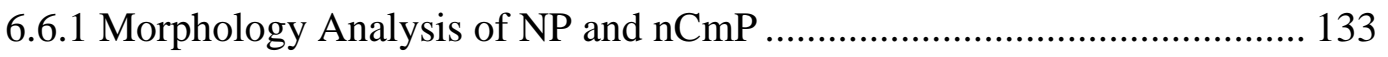

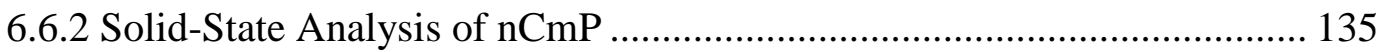

6.6.3 Aerosol Performance Characterization of $\mathrm{nCmP}$........................................ 137

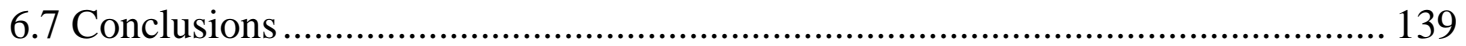

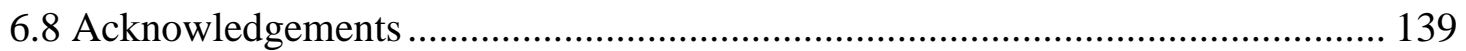

CHAPTER 7 CONCLUSIONS AND FUTURE WORK.................................... 140

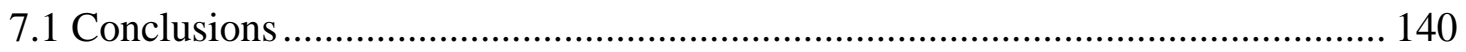

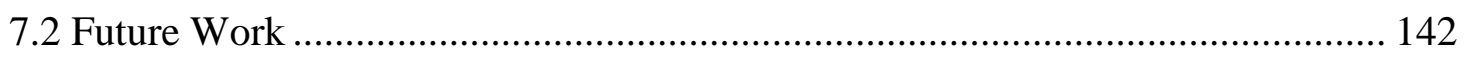

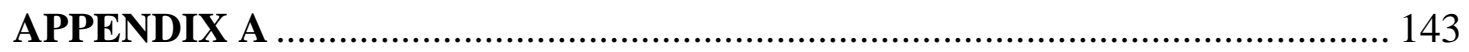

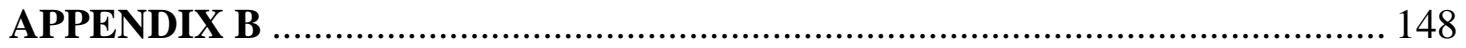

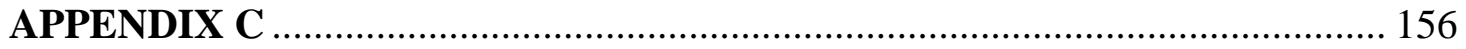

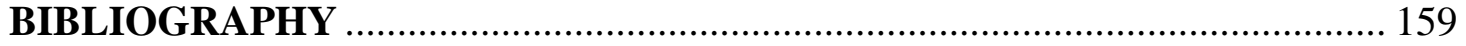




\section{LIST OF TABLES}

Table 3.1. Diameter, polydispersity index (PDI), surface charge via zeta potential, curcumin encapsulation efficiency, and drug loading of nanoparticle system (mean \pm

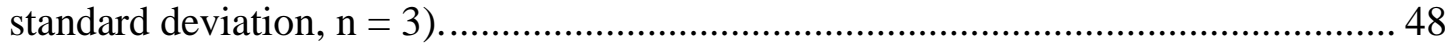

Table 4.1. Spray drying parameters for particle sets, including the reaction time used for acetalated dextran (Ac-Dex) synthesis, spray drying parameters (set inlet temperature and pump rate), and actual inlet and outlet temperatures during the spray drying process. The particle naming convention is as follows: 'Fast' refers to 5-minute Ac-Dex (fast degrading), 'Slow’ refers to 3-hour Ac-Dex (slow degrading), the temperature value is the set inlet temperature setting, and 'LowP', 'MedP', and 'HighP' refers to 10,30 , and $100 \%$ pump rates, respectively (mean \pm standard deviation, $\mathrm{n}=3$ )

Table 4.2. Characteristics of microparticle systems, including (Top) hydrodynamic diameter and polydispersity index (PDI) via dynamic light scattering, tapped density, theoretical mass median aerodynamic diameter $\left(\mathrm{MMAD}_{\mathrm{T}}\right)$ via tapped density calculations, and experimental mass median aerodynamic diameter (MMADEXP) and geometric standard deviation (GSD) via Next Generation Impactor analysis and (Bottom) water content, encapsulation efficiency, and drug loading of sulforhodamine $\mathrm{B}(\mathrm{SRB})$ in the microparticles (mean \pm standard deviation, $\mathrm{n}=3$ ).

Table 4.3. Best-fit drug release kinetics model for each microparticle system, the corresponding fitted parameters, and corresponding coefficient of determination $\left(\mathrm{R}^{2}\right)$ values. $\mathrm{K}_{\mathrm{KP}}$ is the Korsmeyer release rate constant, $\mathrm{n}$ is the drug release exponent, $\mathrm{K}_{1}$ is the first-order release rate constant, $\mathrm{K}_{\mathrm{C}}$ is the first-order rate constant associated 
with kinetics of the burst release for Corrigan, FB, in is the fraction of drug release during burst. The units for the constants are as follows: $K_{K P}[=] h^{-n}, K_{1}[=] h^{-1}, n[=]$ unitless, $\mathrm{K}_{\mathrm{C}}[=] \mathrm{h}^{-1}, \mathrm{~F}_{\mathrm{B}, \mathrm{in}}[=]$ unitless (fraction), $\mathrm{k}_{1}[=] \mathrm{h}^{-\mathrm{m}}, \mathrm{k}_{2}[=] \mathrm{h}^{-2 \mathrm{~m}}, \mathrm{~m}[=]$ unitless.

Table 5.1. (Top) Factors (transmembrane pressure (TMP), flow rate, and PTX initial loading) and levels (low, medium, high) for the Box-Behnken Design of nanoparticle formulations. (Center) randomized processing parameters from Design-Expert. (Bottom) Parameters for optimized nanoparticle formulations used in validation studies $105-106$

Table 5.2. Overview of the influences of the factors (i.e. transmembrane pressure (TMP), flow rate, and initial paclitaxel loading (DL)) on the responses (nanoparticle properties) including the diameter, polydispersity index (PDI), zeta potential, poly(vinyl alcohol) (PVA) content, drug loading (DL) with and without PVA (--- = no significance, $*=p<0.05)$, and their coefficient estimates $(\mathrm{CE})$ 112 Table 6.1. Physical characteristics of Eact-loaded nanoparticles (NP) and nanocomposite microparticles $(\mathrm{nCmP})$ including the diameter, polydispersity index (PDI), surface charge via zeta potential, drug loading, encapsulation efficiency (EE), water content, and PVA surface content (mean \pm standard deviation, $n=3) \ldots \ldots \ldots . .134$ Table 6.2. Aerosolization performance properties of spray-dried Eact-loaded nanocomposite microparticles (Eact $\mathrm{nCmP}$ ), including fine particle dose (FPD), fine particle fraction $(\mathrm{FPF})$, respirable fraction $(\mathrm{RF})$, emitted dose (ED), experimental mass median aerodynamic diameter (MMADEXP), and geometric standard deviation (GSD) via Next Generation Impactor analysis (mean \pm standard deviation, $\mathrm{n}=3$ ) ........... 138 


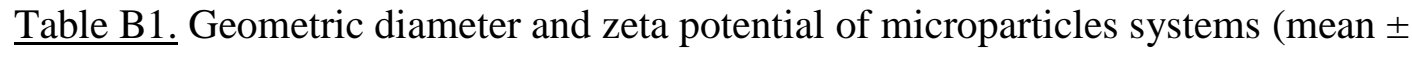

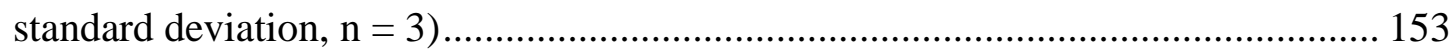

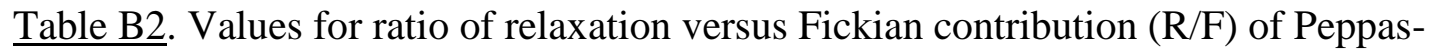
Sahlin drug release kinetics model for MP drug release at $\mathrm{pH} 5 . \mathrm{F} 100 \mathrm{M}=$ Fast $-100^{\circ} \mathrm{C}-$ MedP, F150M $=$ Fast $-150^{\circ} \mathrm{C}-\mathrm{MedP}, \mathrm{F} 100 \mathrm{H}=$ Fast $-100^{\circ} \mathrm{C}-$ HighP, $\mathrm{S} 100 \mathrm{M}=$ Slow$100^{\circ} \mathrm{C}-\mathrm{MedP}, \mathrm{S} 150 \mathrm{M}=\mathrm{Slow}-150^{\circ} \mathrm{C}-\mathrm{MedP}, \mathrm{S} 100 \mathrm{H}=\mathrm{Slow}-100^{\circ} \mathrm{C}-\mathrm{HighP}$ 154

Table C1. Chemical structures and characteristics of the encapsulated model therapeutics paclitaxel (PTX), tacrolimus (TAC), and resveratrol (RSV). Characteristics include molecular weight (MW), partition coefficient (Log P), and water solubility. Predictions for characteristics were calculated using ACDLabs.com

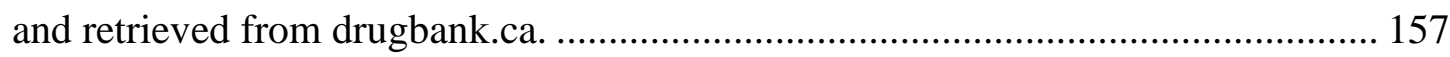




\section{LIST OF FIGURES}

Figure 1.1 Schematic representation of (a) dead end filtration (i.e. centrifugation) and

(b) tangential flow filtration for separation of particulates 3

Figure 1.2 Reaction Schematic of Acetalated Dextran 5

Figure 1.3 Drug delivery technologies manufactured using Ac-Dex 6

Figure 2.1 (A) Routes and delivery locations for parenteral delivery of therapeutics or nanocarriers, including intramuscular, subcutaneous, intravenous, and intradermal delivery methods. (B) Cellular and non-cellular pulmonary barriers for nanocarriers including those present in the central lung/upper airways (bronchial) and peripheral/lower airways (alveoli)

Figure 3.1 Representative SEM (left) and TEM (middle, right) micrographs of PVAand DPPS-coated, CUR-loaded nanoparticles (PVA-CUR NP and PVA-DPPS NP, respectively)

Figure 3.2 Quantification of the amount of poly(vinyl alcohol) (PVA) and total phospholipid in PVA-CUR, DPPC-CUR, and DPPS-CUR nanoparticles (NP) $\left({ }^{*} \mathrm{p}<\right.$ $0.05, * * \mathrm{p}<0.01, * * * \mathrm{p}<0.001$ in comparison to PVA-CUR NP) 50

Figure 3.3 Differential scanning calorimetry (DSC) thermograms of (A) formulated nanoparticles and (B) the raw components that make up the nanoparticle systems in their dry state, and $(\mathrm{C})$ formulated nanoparticles in their wet state

Figure 3.4 Cumulative release of curcumin (CUR) from nanoparticle systems at $\mathrm{pH} 7$ and $\mathrm{pH} 5\left(* \mathrm{p}<0.05, * * \mathrm{p}<0.01,{ }^{* * *} \mathrm{p}<0.001, \mathrm{~ns}=\right.$ non-significant $)$

Figure 3.5 Cytotoxicity analysis (relative viability) of nanoparticle systems on RAW

264.7 macrophages after 48 hours of exposure compared to control $(* \mathrm{p}<0.05) \ldots . . .55$ 
Figure 3.6 Nitric oxide (NO) production from RAW 264.7 macrophages incubated for 48 hours with varying concentrations of nanoparticle formulations or LPS. No statistical significance was observed between the groups and control group (no treatment) and all were statistically lower than the LPS sample $(\mathrm{p}<0.0001)$ 56

Figure 3.7 (Left) Fluorescence spectroscopy analysis of nanoparticle uptake into cells and (Right) representative confocal images of nanoparticle uptake by RAW 264.7 macrophages and A549 cells (scale bar $=10 \mu \mathrm{m})$ 57

Figure 4.1 Schematic of microparticle preparation including mixing of aqueous solution containing sulforhodamine (SRB) and water and organic solution containing acetalated dextran (Ac-Dex) and isopropyl alcohol (IPA) to form a water/organic (W/O) solution prior to spray drying and microparticle formation. 73

Figure 4.2 Representative scanning electron microscopy micrographs of spray-dried sulforhodamine B (SRB)-loaded microparticles. Images were taken at 10,000x. Scale bar $=2 \mu \mathrm{m}$ 82

Figure 4.3 (Top) Aerosol dispersion analysis of fast and slow degrading particles in stages 1-7 of a Next Generation Impactor, expressed as percent particle deposition per stage (mean \pm standard deviation, $n=3$ ). For $Q=60 \mathrm{~L} / \mathrm{min}$, cutoffs for each stage of the NGI are: $8.06 \mu \mathrm{m}$ (stage 1), $4.46 \mu \mathrm{m}$ (stage 2), $2.82 \mu \mathrm{m}$ (stage 3), $1.66 \mu \mathrm{m}$ (stage 4), $0.94 \mu \mathrm{m}$ (stage 5), $0.55 \mu \mathrm{m}$ (stage 6) and $0.34 \mu \mathrm{m}$ (stage 7). (Bottom) Aerosolization performance properties of spray-dried particle systems, including fine particle fraction (FPF), respirable fraction (RF), and emitted dose (ED) (mean \pm standard deviation, $\mathrm{n}=3$ ). 84

Figure 4.4 (A) Representative differential scanning calorimetry thermograms of raw 
materials used during the spray drying of particles and of sulforhodamine B (SRB)loaded microparticles. (B) Representative x-ray diffractograms of raw materials used during the spray drying of particles and of sulforhodamine B (SRB)-loaded microparticles. (C) Polarized light microscopy images of microparticle systems and raw materials. Crystals in microparticle systems are indicated by arrows, if applicable.

Scale bar $=0.1 \mathrm{~mm}$ 86

Figure 4.5 Normalized percent weight release profiles of sulforhodamine-B (SRB) microparticles at $\mathrm{pH} 5$ and $\mathrm{pH} 7$ as a function of time (mean \pm standard deviation, $\mathrm{n}=$

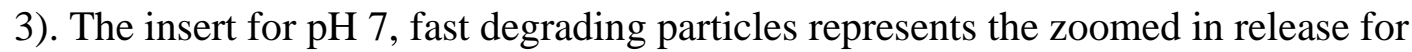
mannitol microparticles.

Figure 4.6 Results from experiments where cells were exposed to sulforhodamine B (SRB)-loaded microparticles (MP), including (A) Transepithelial electrical resistance (TEER) data for H441 cells exposed to microparticle systems from day -9 to day 5 , where day 0 was the particle exposure day. The particle naming convention corresponds to the following: $\mathrm{F} 100 \mathrm{M}=$ Fast $-100^{\circ} \mathrm{C}-\mathrm{MedP}, \mathrm{S} 100 \mathrm{M}=\mathrm{Slow}-100^{\circ} \mathrm{C}-$ MedP, F150M = Fast- $150^{\circ} \mathrm{C}-\mathrm{MedP}, \mathrm{S} 150 \mathrm{M}=$ Slow- $150^{\circ} \mathrm{C}-\mathrm{MedP}, \mathrm{F} 100 \mathrm{H}=$ Fast$100^{\circ} \mathrm{C}-\mathrm{HighP}$, and $\mathrm{S} 100 \mathrm{H}=\mathrm{Slow}-100^{\circ} \mathrm{C}-\mathrm{HighP}$; (B) Viability of A549 cells exposed to $\mathrm{SRB} \mathrm{MP}(* \mathrm{p}<0.05, * * \mathrm{p}<0.01$, mean \pm standard deviation, $\mathrm{n}=3)$; and $(\mathrm{C}) \mathrm{SRB}$ MP uptake by A549, Calu-3, and H441 cell lines $(* \mathrm{p}<0.05, * * \mathrm{p}<0.01$, ***p $<$ 0.001, mean \pm standard deviation, $\mathrm{n}=3$ )

Figure 5.1 Schematic overview of the nanoparticle (NP) purification processes of centrifugation and tangential flow filtration (TFF). For centrifugation, deionized (DI) water was added to the NP suspension, following by centrifugation (Washing, 
Supernatant 1), this step was repeated (Washing, Supernatant 2), and the NP were resuspended in $0.1 \%$ poly(vinyl alcohol) (PVA). For TFF, the NP suspension underwent an initial filtration (Diafiltration, Step 1), then a concentration step occurred following addition of $0.05 \%$ PVA to get $15 \mathrm{~mL}$ of concentration NP (Concentration, Step 2), and finally the TFF membrane was cleaned to collect residual residue (Membrane Cleaning, Step 3). 108

Figure 5.2 Characteristics of paclitaxel loaded nanoparticles (PTX NP) (NP) purified using TFF including Diameter and polydispersity index (PDI); Zeta potential; Poly(vinyl alcohol) (PVA) content; Actual PTX loading (with and without PVA) Mean \pm standard deviation, $\mathrm{n}=3$ 111

Figure 5.3 Data showing the effect of tangential flow filtration (TFF) process parameters on nanoparticle (NP) characteristics. (Top Left) The effect of TFF flow rate on nanoparticle polydispersion index (PDI). (Top Center) The effect of Initial PTX loading on nanoparticles zeta potential. (Top Right) The effect of initial PTX loading on actual PTX loading (with and without poly(vinyl alcohol), PVA). (Bottom) Contour plots showing the effect of TFF flow rate and transmembrane pressure (TMP) on NP diameter at different initial paclitaxel (PTX) loadings. Regions where nanoparticles exhibited diameters of $150 \mathrm{~nm}$ or less on contour maps are indicated by arrows.

Figure 5.4 Characteristics of paclitaxel (PTX), tacrolimus (TAC), and resveratrol (RSV)-loaded nanoparticles (NP) before purification and after tangential flow filtration (TFF) and centrifugation purification processes. ${ }^{*} p<0.05,{ }^{* *} p<0.01,{ }^{* * *} p$ $<0.001$, and $* * * * p<0.0001$. Mean \pm standard deviation, $\mathrm{n}=3$. 115 
Figure 5.5 Representative scanning electron micrographs of paclitaxel nanoparticle formulations purified via (Left) centrifugation and (Right) tangential flow filtration (TFF). Particles are shown with (Top) poly(vinyl alcohol) (PVA) present and (Bottom) in the absence of PVA. Scale bar $=500 \mathrm{~nm}$. 116

Figure 5.6 Drug loading and poly(vinyl alcohol) (PVA) content of paclitaxel, tacrolimus, and resveratrol-loaded nanoparticle (PTX-NP, TAC-NP, and RSV-NP, respectively) suspensions taken during the centrifugation and tangential flow filtration purification processes. ${ }^{*} p<0.05,{ }^{* *} p<0.01,{ }^{* * *} p<0.001$, and ${ }^{* * * *} p<0.0001$. Mean \pm standard deviation, $\mathrm{n}=3$.

Figure 6.1 SEM micrographs of raw Eact, parent Eact nanoparticles (NP), Eact nanocomposite microparticles (nCmP), and $\mathrm{NP}$ re-dispersed from $\mathrm{nCmP}$. 134 Figure 6.2 (A) Representative x-ray diffractograms, (B) representative differential scanning calorimetry thermograms and (C) polarized light microscopy images of raw materials (3-hour Ac-Dex and Eact) and Eact-loaded nCmP. Scale bar $=0.1 \mathrm{~mm} . .136$ Figure 6.3 Aerosol dispersion analysis of Eact-loaded nanocomposite microparticles in stages 1-7 of a Next Generation Impactor, expressed as percent particle deposition per stage (mean \pm standard deviation, $n=3$ ). For $Q=60 \mathrm{~L} / \mathrm{min}$, cutoffs for each stage of the NGI are: $8.06 \mu \mathrm{m}$ (stage 1), $4.46 \mu \mathrm{m}$ (stage 2), $2.82 \mu \mathrm{m}$ (stage 3), $1.66 \mu \mathrm{m}$ (stage 4), $0.94 \mu \mathrm{m}$ (stage 5), $0.55 \mu \mathrm{m}$ (stage 6) and $0.34 \mu \mathrm{m}$ (stage 7).............................. 138 Figure A1. Chemical Structures of DPPS (A), Ac-Dex (B), and Curcumin (C) ..... 144 Figure A2. NMR Spectra of Ac-Dex with normalization integrations relating to associated peaks of methanol, acetone, and carbon ring of dextran 
Figure A3. Wet state DSC of the nanoparticle systems

Figure A4. Diameter (left) and zeta potential (right) of Blank-PVA nanoparticle (A), Blank-DPPS nanoparticle (B), CUR-PVA nanoparticle (C), and CUR-DPPS nanoparticle (D) systems.

Figure B1. Total particle mass (mg) of aerosolization performance properties of particles, including fine particle fraction (FPF), respirable fraction (RF), and emitted

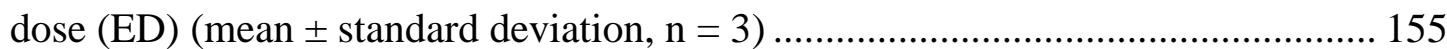




\section{CHAPTER 1}

\section{INTRODUCTION}

\subsection{Motivation}

Following the introduction of particle-based delivery systems, the field of drug delivery has significantly improved the pharmacological and pharmacokinetic outcomes of therapeutics by decreasing drug toxicity and increasing bioavailability $(1$, 2). Drug formulations that involve particle-based technology provide multiple advantages over conventional dosage forms $(2,3)$. By using particle-based delivery systems, drug formulations experience a decrease in overall size (several to 100's of microns to single microns or nanometer diameters), allowing for an increase in surface-area-to-volume ratio, providing an increase in dispersion, absorption, and overall bioavailability $(4,5)$. Utilizing particle size and composition, particle-based delivery systems can passively target tissues using endogenous uptake phenomena (6). For example, passive targeting has been shown to decrease drug toxicity, particularly for cancer, through the use of enhanced permeability and retention (EPR) effect (711). An example of this occurrence is the mechanistic enhanced uptake of Doxil ${ }^{\circledR}$, liposomal doxorubicin, into cancerous tissue $(12,13)$.

Although drug toxicity and bioavailability are major obstacles that need to be overcome using particle-based delivery systems, the former is still a concern. Major causes of drug toxicity relate to unwanted drug deposition, unfavorable release kinetics/mechanisms, and less ideal routes of administration (i.e. oral delivery for pulmonary disease states) $(2,14,15)$. As a result, there has been a rise in the 
development of novel strategies to increase the quality of drug delivery systems to enhance the effectiveness of disease treatment while decreasing the likelihood of drug toxicity (2). Strategies to improve particle-based technologies in this regard include $i$ ) surface targeting mechanisms (peptides, small molecules, antibodies, etc.) (16-18) (drug targeting); ii) external stimulation (radio frequency, ultrasound, ultraviolet light, etc.), environmental stimulation (oxidation, $\mathrm{pH}$, enzymatic processes, etc.) and the development and utilization of novel polymers (19) (controlling release kinetics); and iii) developing new strategies for delivery (routes of administration).

Another concern is related to production of particle technologies, particularly nano-sized technologies. With the exceptional growth and promise of particle technology, scale-up synthesis of these systems has been a manufacturing interest (2022). However, an often overseen yet vital aspect related to manufacturing is product purification. Prior to packaging and usage, drug products must be purified, to ensure the highest quality of the product (23). However, the purification of continuously manufactured and scaled-up batches of particle technologies has reached an impasse (24). Currently, the most often used purification method for particle technologies is centrifugation. Although this method is widely used and is highly regarded as an effective way to purify particle-based systems, there are major concerns with respect to scalability (23). Additionally, certain effects, such as the gravitational forces used in centrifuges, can have major impacts on the outcomes of NP characteristics (24). One potential solution is the usage of tangential flow filtration (TFF), which has quickly gained praise as a new method of scale-up purification for a variety of particle-based technologies (25-34). Unlike centrifugation, TFF utilizes fluid dynamics to decrease 
the likelihood of aggregation by preventing gravitational force-related caking, while continually washing and concentrating NP suspensions (Figure 1.1) (23). This is achieved when fluid containing solutes flows tangentially to a replaceable filter. Despite its use and promise, there has not been an investigation on the key parameters of TFF to study the influence on NP characteristics. A study such as this would allow further understanding of TFF's promise as a purification method as well as how the parameters impact the outcomes of NPs.

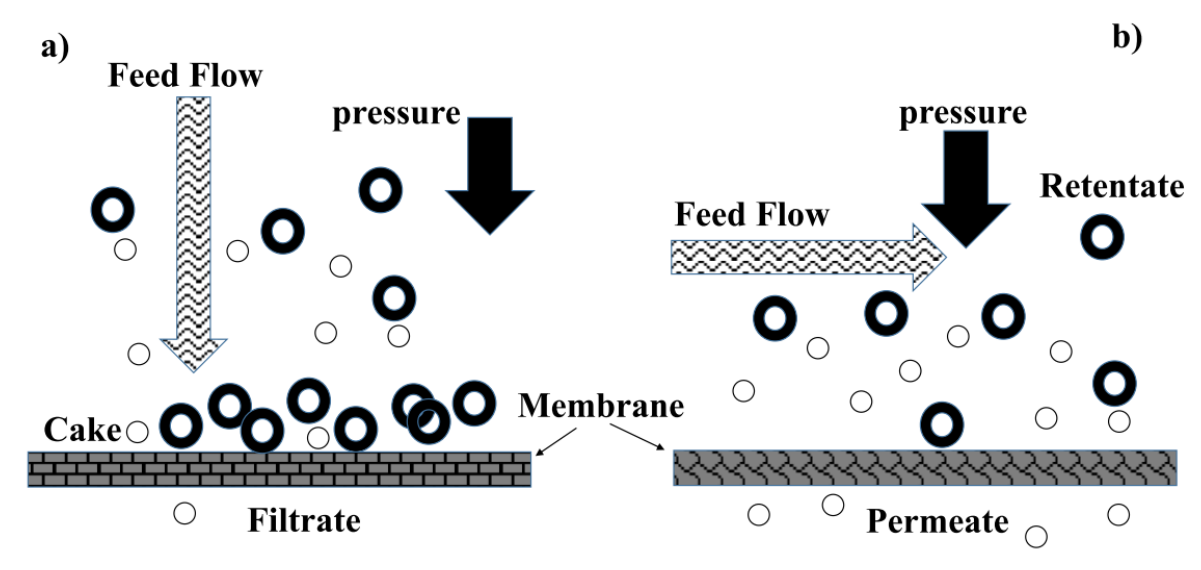

Figure 1.1 Schematic representation of (a) dead end filtration (i.e. centrifugation) and (b) tangential flow filtration for separation of particulates (23).

Polymers, which are the most often utilized type of drug carrier, continually present opportunities to advance particle-based technologies (2). The physical construction of polymers can be tuned and utilized to take advantage of passive targeting, to modify release kinetics, and to allow for targeting modification such as antibodies, small molecules, etc. $(35,36)$. In some cases, polymers can be naturally occurring or can be synthetic derivatives of natural polymers (biopolymers). Examples of biopolymers include chitosan, alginate, and peptides (36-39). Polysaccharides can also be utilized as drug carriers, often following chemical modification (19). From a 
product standpoint, the use of polymers allows for the stable and robust production of drug carriers that can be tailored for both the patient population and the intended disease state.

Acetalated dextran (Ac-Dex) is a synthetic derivative of dextran that was first synthesized a decade ago for drug delivery applications (40). Dextran, a water-soluble polysaccharide, is converted to water-insoluble Ac-Dex through a cost-effective, facile one-step reaction (Figure 1.2) $(36,40)$. The characteristics of Ac-Dex allow it to be a suitable polymer for the sustained delivery of therapeutic agents. The polymer is $\mathrm{pH}-$ sensitive (acid labile), degrades into dextran and safe amounts of methanol and acetone, and is considered to be non-toxic, as seen in in vivo studies) $(41,42)$. In addition, Ac-Dex is tunable with respect to its degradation rate, where the influences of dextran molecular weight and reaction time impact the release kinetics of therapeutics loaded in Ac-Dex particle-based formulations. It has been shown in numerous studies that increases in both molecular weight and reaction time can slow release of encapsulated cargo $(35,43)$. The tunability of its degradation rate, combined with its acid-labile nature, make Ac-Dex an attractive polymer for triggered release at the site of cancer and infectious diseases, owing to their acidic microenvironments in addition to minimal release in circulation due to particle stability at physiological $\mathrm{pH}$. 


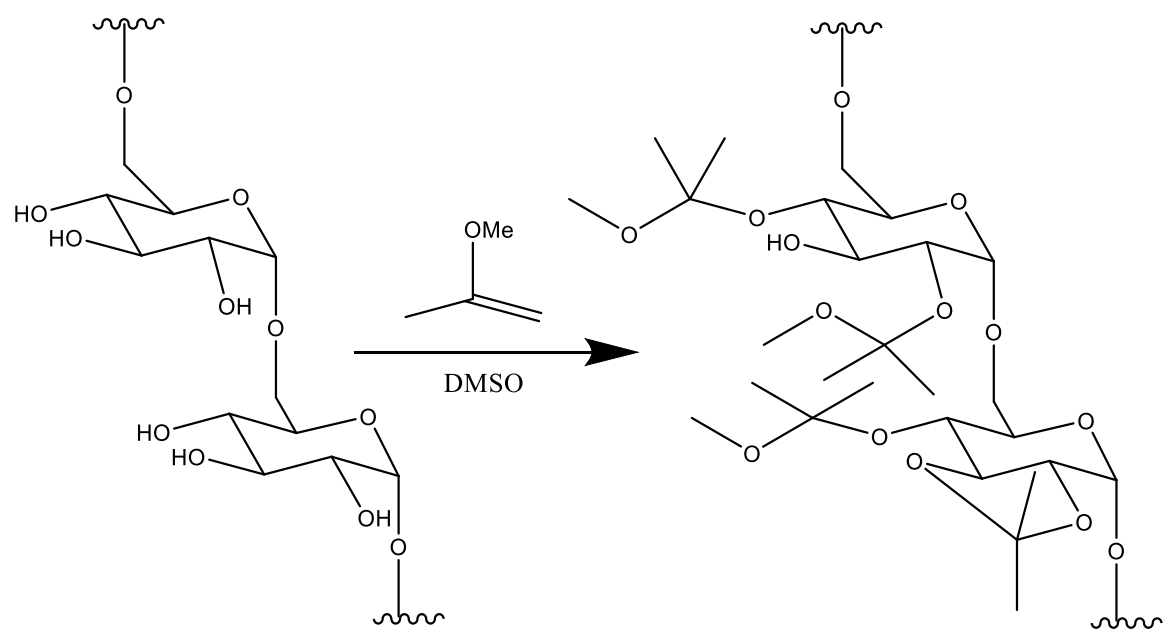

Figure 1.2. Reaction schematic of acetalated dextran.

In total, Ac-Dex has been utilized in as many as 50 manuscripts, including an extensive review recently published in 2017 (19). Drug delivery technologies that have been created using Ac-Dex include porous microparticles, nanocomposite microparticles, nanoparticles, microconfetti, electrosprayed microparticles, nanocomposite microparticles and polymeric scaffolds (Figure 1.3) $(19,44,45)$. Therapeutic cargos of both small and large molecular weights have been encapsulated in one or more of these systems and have been used for autoimmune diseases (42), vaccination (46-48), cancer $(49,50)$, infectious diseases $(41,51,52)$, pulmonary diseases (53, 54), and tissue engineering (19). 


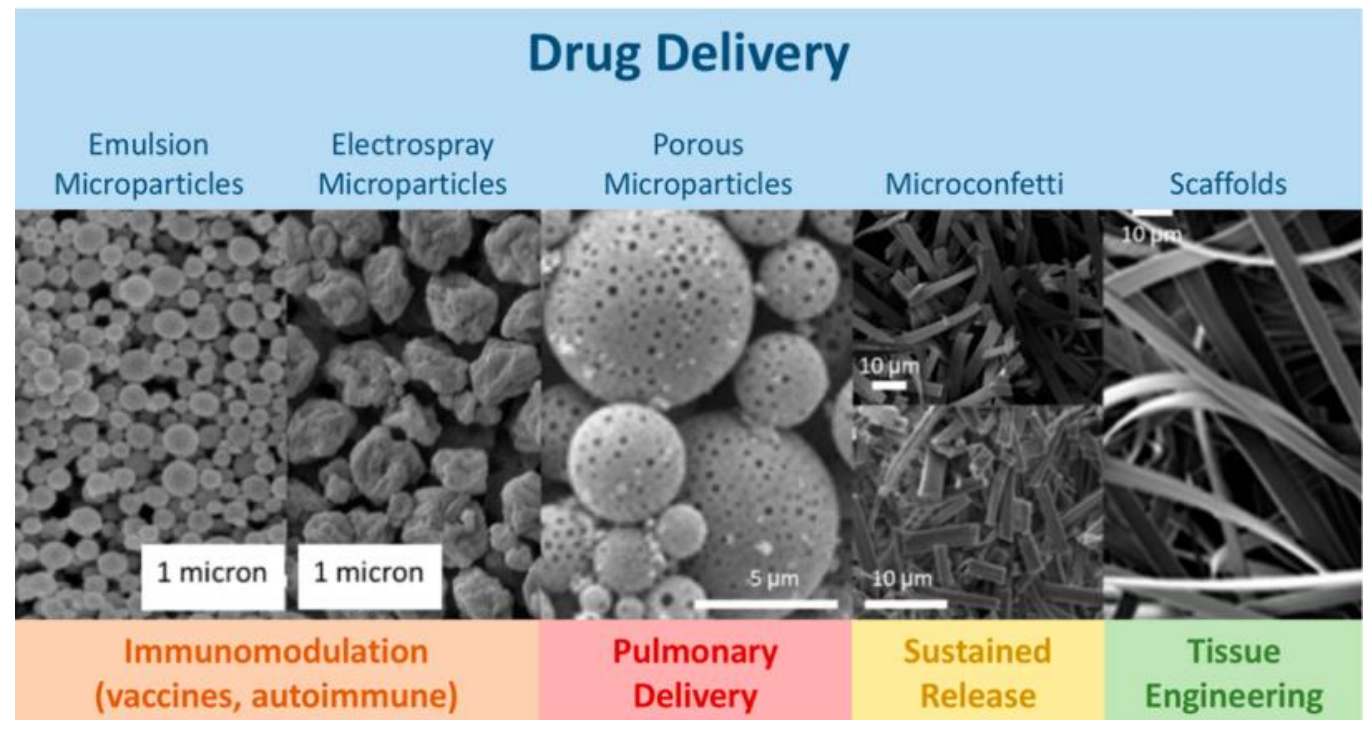

Figure 1.3 Drug delivery technologies manufactured using Ac-Dex. Adopted from (19).

Although Ac-Dex has shown promise as a versatile and cost-effective biopolymer, there are still knowledge gaps that require exploration. In this dissertation, research has been devoted to assessing the unexplored territories of Ac-Dex as a drug carrier to combat current drug delivery obstacles. In Manuscript 1, Ac-Dex nanoparticles (NP) were coated in phosphatidylserine (DPPS) to mimic apoptotic cells for active macrophage targeting. Following the synthesis and characterization of the DPPScoated NP, phospholipid coverage was confirmed using TEM and the Stewart's assay. Finally, for biological confirmation, DPPS-coated NP were compared to DPPC/Gcoated NP and PVA-coated NP via their in vitro uptake into macrophages. In Manuscript 2, multiple types of Ac-Dex microparticles (MP) synthesized via spray drying were evaluated for their aerosol performance and were assessed for the release kinetic behavior of a water-soluble dye. Manuscript 3 evaluates a broader obstacle in particle technology. Using a design of experiment (DoE) approach, the effects of key parameters of tangential flow filtration (TFF) on the characteristics of Ac-Dex NP 
were studied to understand the promise of TFF as a scale-up purification technique. The final manuscript evaluated the physical characteristics of Eact, followed by the development of Ac-Dex nanocomposite microparticles (nCmPs), to achieve deep lung deposition for the treatment of pulmonary arterial hypertension (PAH). In total, these manuscripts were designed and carried out with the intention of continuing the research devoted to Ac-Dex delivery formulations to provide further evidence that it is an efficient, versatile biopolymer for a variety of drug delivery applications.

\subsection{Significance of This Work}

The manuscripts in this dissertation are centered around the continued exploration of the biodegradable polymer Ac-Dex and its versatility as a drug carrier to overcome obstacles in drug delivery. The significance of this research is listed below.

1. Ac-Dex nanoparticle systems can be tailored to actively target specific sites for diseases that would otherwise difficult to treat.

2. Ac-Dex microparticle systems can be modified via facile tunability to modulate the drug release kinetics of payloads that are difficult to control.

3. Ac-Dex nanoparticle systems can be purified using scale-up technology, allowing for large-scale production and more favorable nanoparticle characteristics, which can lead to increases in manufacturing efficiency. 


\subsection{Objectives of the Research}

The overall objective of this dissertation was to develop and characterize Ac-Dex particle-based technologies for drug delivery applications in order to explore and identify specific challenges that Ac-Dex can overcome, as well as explore boarder challenges for drug delivery to continue to show the versatility and promise of the biopolymer. In this regard, each manuscript has its own specific objectives:

1. "Enhancement of Macrophage Uptake via Phosphatidylserine-Coated Acetalated Dextran Nanoparticles":

a. Prepare Ac-Dex nanoparticles (NP) loaded with a curcumin (CUR) for ease of quantification.

b. Successfully coat Ac-Dex NP with phosphatidylserine (PS) and characterize the systems for their size, homogeneity, surface charge, and presence of coatings.

c. Evaluate the drug release profiles of CUR-loaded PS NP at neutral and acidic $\mathrm{pH}$.

d. Evaluate the in vitro phagocytotic uptake of Ac-Dex PS NP into RAW 264.7 murine macrophages.

2. "Sustained Release of Water-Soluble Therapeutics via Dry Powder Aerosol Acetalated Dextran Microparticles" 
a. Synthesize Ac-Dex microparticle (MP) systems encapsulating sulforhodamine B via various spray-drying conditions and two types of Ac-Dex (fast and slow degrading).

b. Evaluate the physical and aerosol characteristics of the MP formulations.

c. Examine the drug release profiles, and model the release kinetics of MP systems at neutral and acidic $\mathrm{pH}$.

d. Examine biological interactions in vitro via cytotoxicity, uptake, and transepithelial electrical resistance studies.

3. "Comparison of Nanoparticle Purification Process via Centrifugation and Tangential Flow Filtration: A Case Study of Acetalated Dextran Nanoparticles"

a. Utilize Design of Experiment to study key parameters of nanoparticles (NP) with respect to tangential flow filtration (TFF) purification.

b. Compare properties of TFF-purified NP to centrifugation-purified NP.

c. Evaluate the variability in NP characteristics following purification by TFF when varying small molecules are encapsulated in NP systems.

4. "Physical Characterization and Formulation Development of Eact for the Treatment of Pulmonary Arterial Hypertension”

a. Physical characterization of Eact.

b. Synthesis and characterization of Eact-loaded nanoparticles. 
c. Synthesis of Eact $\mathrm{nCmP}$ via spray drying, and evaluation of physical characteristics and aerosol performance of $\mathrm{nCmP}$ formulations.

\subsection{Organization of Dissertation}

The remainder of this dissertation will be organized as follows:

Chapter 2 provides a background on particle-based technology, as well as parenteral delivery and pulmonary routes of administration. The latter two sections (routes of administration) are partially adopted from Chapter 6 of the book "Nanoparticles for Biomedical Applications" (55), which is currently under review for publication.

Chapter 3 describes the work related to the manuscript, "Enhancement of Macrophage Uptake via Phosphatidylserine-Coated Acetalated Dextran Nanoparticles" (52), which involves the design and development of phospholipidcoated Ac-Dex nanoparticles capable of actively targeting and being uptake by macrophages.

Chapter 4 describes the work related to the manuscript, "Sustained Release of Water-Soluble Therapeutics via Dry Powder Aerosol Acetalated Dextran Microparticles," which involves the synthesis of Ac-Dex microparticles via spray drying, and the observation of their drug release behaviors and aerosol performance.

Chapter 5 describes the work related to the manuscript, "Comparison of Nanoparticle Purification Process via Centrifugation and Tangential Flow Filtration: A Case Study of Acetalated Dextran Nanoparticles," which involves the use of Design of 
Experiments to identify and study the effects of key tangential flow filtration parameters (factors) on Ac-Dex nanoparticle characteristics (responses).

Chapter 6 presents data related to the "Physical Characterization and Formulation Development of Eact for the Treatment of Pulmonary Arterial Hypertension," which involves the design and characterization of nanocomposite microparticles encapsulating Eact, a small molecule currently under investigation for its therapeutic potential for treating pulmonary arterial hypertension.

Chapter 7 concludes the dissertation and addresses future problems and future directions of Ac-Dex-based drug delivery formulations. 


\section{CHAPTER 2}

\section{PARTICLE TECHNOLOGY AND ROUTES OF ADMINISTRATION:}

\section{PARENTERAL AND PULMONARY DELIVERY}

Partially adopted from Chapter 6 of "Nanoparticles for Biomedical Applications: Fundamental Concepts, Body Interactions, and Clinical Potential.” Editors: Eun Ji Chung, Lorraine Leon, Carlos Rinaldi; Elsevier Science \& Technology; Amsterdam, The Netherlands; In publication March 2019.

\footnotetext{
Nishan K. Shah ${ }^{1}$, Elisa A. Torrico ${ }^{2}$, Zimeng Wang ${ }^{3}$, Samantha A. Meenach ${ }^{1,2, *}$

${ }^{1}$ Department of Biomedical and Pharmaceutical Sciences, College of Pharmacy, University of Rhode Island, Kingston, RI 02881 USA

${ }^{2}$ Department of Chemical Engineering, College of Engineering, University of Rhode Island, Kingston, RI 02881 USA

${ }^{3}$ Phosphorex Inc., Hopkinton, MA 01748
} 


\subsection{Particle Technology}

The use of particle-based technologies have significantly advanced the fields of electronics, food, industrial development, and medicine (56). Within the medical field, particle technologies have made enormous strides as diagnostic and therapeutic agents, and therapeutic delivery systems. Through particle engineering, particle-based technologies exhibiting specific size, shape, surface charge, and physicochemical characteristics can be developed for a variety of disease states or imaging techniques (57).

Microparticles (MP) are used for depot injections, as inhalable powders, and as therapeutic agents. Depot injections such as Risperdal Consta ${ }^{\circledR}$ and Invega Trinza ${ }^{\circledR}$ have shown great success in treating patients with mental health disorders, including schizophrenia and bipolar disorder $(58,59)$. Additionally, formulations for depot injections have been approved for treating diseases such as cancer and alcohol dependence (59). Dry powder aerosols are a type of MP intended for pulmonary administration to treat numerous diseases. $\mathrm{TOBI}^{\circledR}{ }^{\text {Podhaler }}{ }^{\mathrm{TM}}$ is an example of a dry powder aerosol formulation that is used for the treatment of cystic fibrosis-related infections (60). Through the usage of PulmoSphere ${ }^{\mathrm{TM}}$ technology, dry powder aerosols take the form of spherical, porous MP, allowing for effective delivery (61). MP have also shown success as therapeutic agents. One example is Sebacia Microparticles ${ }^{\circledR}$ for the treatment of acne, which was approved for clinical use in 2018 (62). Following topical administration of a suspension, gold-coated silica MP are heated with a laser, which induces thermal injury to the targeted sites without inducing a major inflammatory response (63). Another example is Yttirum-90 
microspheres, which have been explored as a possible alternative approach for cancer treatment (64).

Nanoparticles (NP) range from $1 \mathrm{~nm}$ to under 1 micron. These systems are often used as drug delivery systems, therapeutics, and diagnostic tools. As of 2016, a total of 13 products including liposomal (Doxil ${ }^{\circledR}$ ), nanocrystal (Rapamune ${ }^{\circledR}$ ), polymeric $\left(\right.$ Copaxone $\left.{ }^{\circledR}\right)$, albumin-bound nanoparticle $\left(\right.$ Abraxane $\left.^{\circledR}\right)$, and inorganic $\left(\right.$ Feraheme $^{\circledR}$ ) formulations have been approved for use by the FDA as NP-based systems for therapeutic purposes $(1,65,66)$. From 2013 to 2016 the number of clinical trials involving nanotechnology has increased 3-fold and it is anticipated that this trend will continue, with an overall increase in FDA approvals (1). Thus far, all FDA approved nano-based technologies have been "simple" (NP core plus a coating) and have been based on usage of biological processes and endogenous phenomena for particle uptake and deposition. However, since nanotechnology continues to build from previous successes and failures, it is likely that more complex formulations (external stimuli, active targeting via ligands and antibodies, etc.) will soon be in clinical trials (1).

Although particle technology has existed for a substantial amount of time, there is still a tremendous amount of funding and research devoted to designing and developing technologies for the treatment and diagnosis of numerous disease states (65). Included in their design are ideologies of the specific routes of administration that may be used for delivery (57). In general, particle-based technologies have been explored in almost every administration route, with parenteral routes being the most common (1). However, the pulmonary route is gaining more interest due the ability to 
localize delivery, increase compliance, and increase treatment efficacy, while decreasing both drug metabolism and systemic side effects. The following sections below, partially adopted from the book "Nanoparticles for Biomedical Applications" describe both parenteral and pulmonary routes of administration for particle-based technologies that pertain to this dissertation.

\subsection{Parenteral Delivery}

Parenteral delivery is the administration of a therapeutic that goes through biological tissues directly to blood vessels, organs, or tissues $(67,68)$. This type of delivery offers advantages including high bioavailability, and in some cases, exceptional therapeutic absorption $(68,69)$. This method can be utilized for patients who cannot take oral formulations due to limitations (e.g. sickness, obstruction, loss of consciousness, etc.). These routes of administration are often favorable for patients experiencing disease states that require immediate therapy, and for drug monitoring by medical personnel $(68,70-73)$. The types of parenteral delivery routes that will be discussed in this chapter include intravenous, intramuscular, intraperitoneal, and subcutaneous routes (Figure 2.1A). 


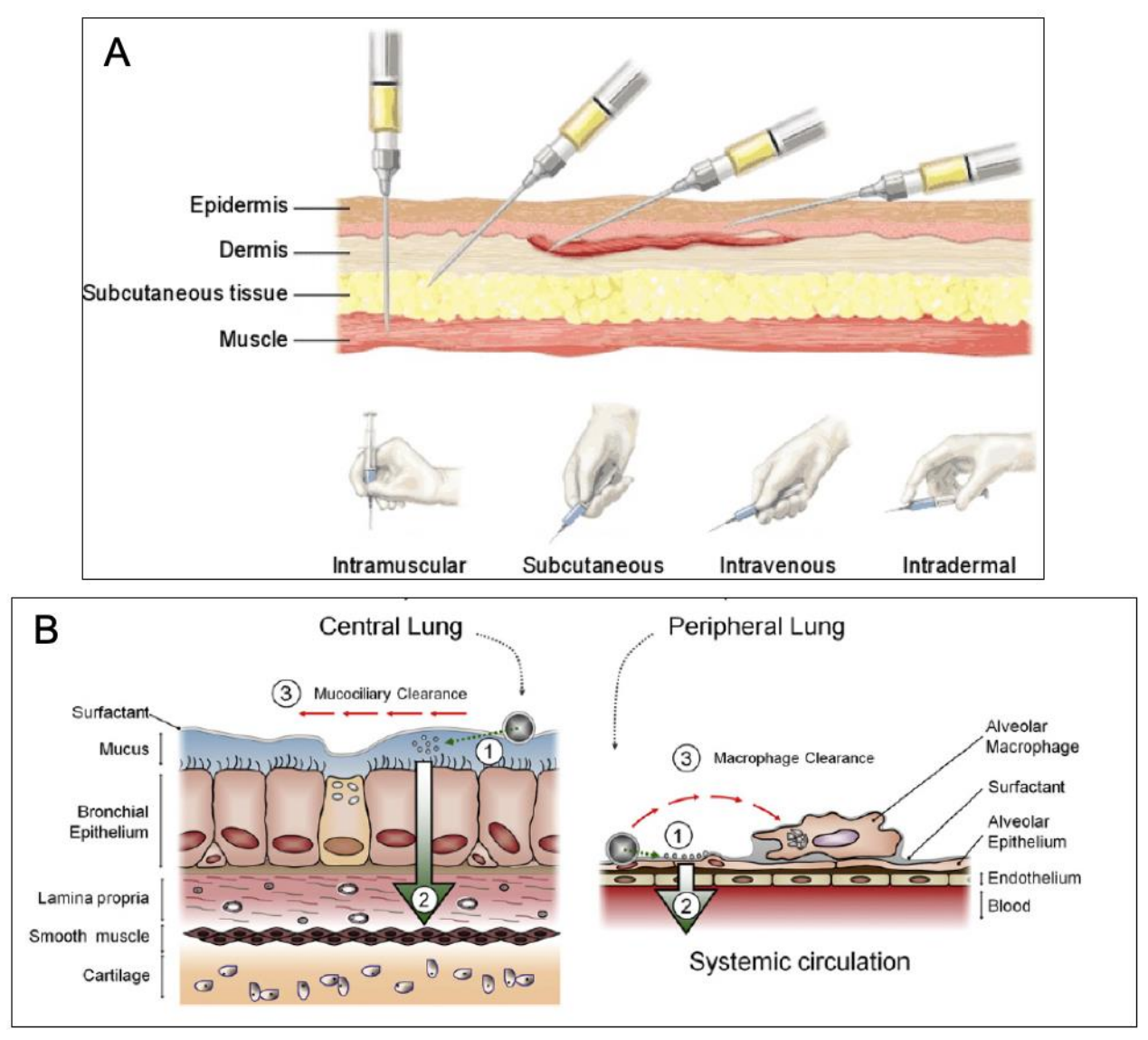

Figure 2.1 (A) Routes and delivery locations for parenteral delivery of therapeutics or nanocarriers, including intramuscular, subcutaneous, intravenous, and intradermal delivery methods. (B) Cellular and non-cellular pulmonary barriers for nanocarriers including those present in the central lung/upper airways (bronchial) and peripheral/lower airways (alveoli). Adapted from (74) and (75).

\subsubsection{Intravenous Delivery}

Aside from oral administration, intravenous (IV) delivery is the most commonly used route of administration in the clinic and involves the delivery of nanocarriers into veins (1). Dosing is performed using either bolus or infusion delivery. The fate of IV-delivered nanocarriers is based on the physical makeup of the 
product (active ingredient and excipients). Alterations to the product and its characteristics will determine overall outcomes. Products administered by IV administration are subject to numerous biological challenges that stem from their physical characteristics including size, charge, shape, and surface modifications (57, 76). Within the body, nanocarriers are subject to protein adsorption, rapid elimination by the reticuloendothelial system (RES), unwanted tissue deposition, and restricted access by biological barriers $(68,77,78)$. Size plays a key role in the biological elimination of nanocarriers, where carriers $<10 \mathrm{~nm}$ are small enough to be filtered and cleared by renal clearance, whereas carriers $>200 \mathrm{~nm}$ are susceptible to the RES, leading to a decrease in therapy (78). Charge also plays a key role in the interactions of nanocarriers with circulating proteins and phagocytotic cells $(79,80)$. Highly negative carriers are susceptible to macrophage uptake, whereas positively-charged products are likely to aggregate with circulation proteins and cellular membranes and are often toxic. Therefore, neutrally-charged formulations are usually overall favorable, unless a specific biological action utilizing charge is desired (i.e. macrophage uptake) $(57,79,80)$. Surface modifications will also have a significant impact on the biological end-point of nanocarriers $(57,81,82)$. Numerous studies have shown unfavorable pharmacokinetic (PK) profiles and unwanted deposition based on the lack of targeting or other forms of modification $(77,78,83)$. Although there is evidence to support nanocarrier shape plays a role in biological interactions, research is still ongoing (84).

The major advantage of IV administration is based on the direct delivery of products into circulation (70), which allows for increased bioavailability. In 
comparison to IV delivery, orally-administered carriers are exposed to enzymes that cause degradation of drugs, acidic $\mathrm{pH}$, and mucus. IV administration of products avoids complications related to degradation, allowing for more effective delivery of sensitive carriers $(69,70)$. In addition, IV delivery is advantageous for carriers that require careful drug monitoring $(68,77,85)$.

\subsubsection{Applications}

Finding ways to predict biological outcomes of nanocarriers in vitro has been a subject of interest for many years. For example, one study investigated the interactions of nanocarriers and blood components and showed a simple yet detailed method to measure the influence of protein adsorption, coagulation, complement activation, and hemolysis on nanocarrier characteristics (76). Another study utilized centrifugation as a method to examine cellular membrane binding and disruption in the characteristics of nanocarriers (86). The development of in vitro experiments such as these can provide time and cost-efficient ways to predict outcomes of nanocarriers in vivo. In addition, research on the impact of surface modification of nanocarriers has been a subject of interest in order to increase the targetability of nanocarriers, to increase pharmacokinetic profiles and decrease side effects. The most successful modification to date is antibody $(\mathrm{Ab})$ conjugation, and clinical trials exploring $\mathrm{Ab}$-functionalized nanocarriers are underway (77).

Both polymeric and liposomal nanocarriers used for chemotherapy have had some success in the clinic and are primarily administered via IV, and the majority of FDA-approved liposomal formulations are administered via IV (87). Improvement of 
PK profiles compared to drug suspensions and a decrease in toxicity have been major advantages of chemotherapeutic nanocarriers (68). Furthermore, the enhanced permeability and retention (EPR) effect is another benefit of IV administration. The proposed mechanism is based on "leaky" blood vessels present in and around tumors, which allow for nanosized material to more readily reach the tumor tissue and exert their therapeutic action $(71,77,85,88)$. To facilitate EPR, nanocarriers are often coated with a hydrophilic, neutral polymer that can allow the nanocarrier avoiding protein aggregation on the surface of the carrier, reducing clearance in the RES $(1,8$, 85). Poly(ethylene glycol) (PEG) is the most widely used hydrophilic polymer for nanoparticle coatings $(78,83)$ and PEG coatings are used in several FDA-approved nanomedicine formulations (e.g. Doxil $\left.{ }^{\circledR}\right)(8,87)$. Another FDA-approved cancer nanocarrier formulation is Abraxane ${ }^{\circledR}$, a third-generation paclitaxel (PTX) delivery formulation that consists of PTX bound to human serum albumin, the most common circulation protein $(71,87)$. Abraxane ${ }^{\circledR}$ has shown significant improvements in administration time (decrease from 3 hours to 30 minutes), as well as improvements in critical PK characteristics in comparison to Taxol®, the commonly used PTX formulation (71).

\subsubsection{Obstacles and Applications}

IV administration is one of the most-widely used administration routes and the main administration route for anti-cancer therapeutics $(71,77,88)$. Despite widespread studies investigating colloidal science and biological interactions $(76,86,89)$, more studies are needed to further identify and counter potential hazards of IV-delivered 
nanocarriers. Other obstacles to consider are more technical. Scale-up synthesis and high-throughput screening methods need to be designed to combat the issue of supply and time-cost investment. An example of this includes product shortage such as the current Doxil ${ }^{\circledR}$ shortage, which needs to be avoided (77). Based on the major obstacles, there are plenty of opportunities for the design of high-throughput screening methods, scale up manufacturing, and predictive tools.

\subsubsection{Intraperitoneal Delivery}

Intraperitoneal (IP) delivery involves the delivery of therapeutics to the peritoneum cavity to increase local concentrations (90). The major use of IP delivery has been for the treatment of peritoneal cancers. The fate of nanocarriers administered via the IP route is highly dependent on the localization of the malignant tissue and the deposition of the administered product in relation to vital organs (91). IP administration generally during or post-surgery (92) and deposition of nanocarriers are initially localized at the administration site, while deviation from the site is affected by the physical characteristics and surface modifications of the formulation, and the dynamics of the site itself including fluid dynamics, gravity, presence of mucus, etc. (93).

In addition to avoiding the stomach, intestines, and first-pass metabolism, the major advantage of IP delivery is the superior level of treatment over IV administration, likely due to the close proximity of therapeutics to malignant tissue (92). IP delivery has been used in the clinic for over 50 years and has demonstrated that it is very efficient in destroying cancerous tissue post-surgery (94). Also, IP 
administration results in reduced levels of systemic exposure $(91,94,95)$. Although IP administration of nanocarriers offer several advantages, challenges are inevitable. Major challenges are the side effects associated with IP delivery, including pain, discomfort, and systemic toxicity (96). While side effects such as pain and discomfort are likely unavoidable, systemic toxicity needs to be addressed by designing nanocarriers that can remain localized. Also, traditional chemotherapeutics are subject to short residence times and require frequent or continuous administration, which can increase side effects and the likelihood of toxicity $(94,96,97)$.

\subsubsection{Applications, Obstacles, and Opportunities}

To address some of challenges pertaining to IP administration, the development of formulations to improve the localization of IP therapeutics is a promising approach. One such method is the development of adhesive formulations that can adhere to the peritoneum, such as chitosan-coated nanocarriers (97). Another method would be the inclusion of nanocarriers in biological mimics, such as hydrogels $(94,96)$. Nanocarriers with targeting ligands, that includes antibodies and peptides, are also being explored $(90,98,99)$ and formulations that exhibit controlled release have also shown promise as novel approaches (94). Another approach that can be used to complement currently-used clinical treatments include nanocarrier-driven hyperthermic intraperitoneal chemotherapy (HIPEC), and pressurized intraperitoneal aerosol chemotherapy (PIPAC). Traditional HIPEC involves the administration of preheated chemotherapeutic solution to a site of interest (92). In doing so, the increased temperature allows for increased drug penetration and therapeutic efficacy $(90,93$, 
99). Clinically, HIPEC has shown to increase the survival rate when used in conjunction with surgery (100). PIPAC, a newer treatment option, utilizes the nebulization of therapeutic solutions (both raw drug and formulations) using $\mathrm{CO}_{2}(93$, 99, 101). To assist in both HIPEC and PIPAC, nanocarriers can be used to control solution and tissue heating, and increase drug deposition via passive and active targeting $(93,99,101,102)$. As of 2017, nanoparticle formulations developed for the treatment of IP-related cancers are in clinical trials (93). A phase I study of IPadministered formulated paclitaxel (Nanotox $\left.{ }^{\circledR}\right)$ showed higher and prolonged drug levels within the peritoneum, resulting in minimal toxicity and systemic drug exposure following six doses of $\operatorname{Nanotax}^{\circledR}$ administered for six cycles (95). A form of HIPEC that utilizes nanocarriers through the use of external heating of inorganic materials to enhance delivery and penetration is currently being studied as well $(99,103)$. In addition, bio-adhesive systems such as hydrogels and nanocarriers have also shown promise to increase the residence time of therapeutics and decrease nanoparticle clearance from the peritoneum $(94,96,97)$.

\subsubsection{Intramuscular Delivery}

Intramuscular (IM) administration involves the delivery of a therapeutic within a muscle and is the most common route used for the delivery of vaccines (104). In addition, IM delivery is often explored as a route for depot products such as hydrogels and microspheres $(105,106)$. The deposition of IM-administered products is heavily dependent on the overall immune response at the delivery site. With respect to the use of IM delivery for vaccines, immune responses are preferred $(107,108)$. However, an 
immune response following administration of formulations that result in the extended release of small molecules is undesirable. Inflammation can result in the recruitment of various white blood cells, which can pose a threat to small molecule formulations, as they would be recognized as foreign objects and would be engulfed (109).

Like most parenteral delivery systems, invasiveness, pain, and stress are major drawbacks for IM delivery. Additionally, IM administration can be associated with severe pain, immune responses (rashes, erythema, inflammation, etc.), and in severe cases, tissue necrosis (110). In most cases, acute edema (swelling) at the site of injection occurs. Due to the interest in long-acting injectables with IM being the preferred route, attention needs to be paid to ensure that prolonged and stable release is achieved for the desired time $(106,110)$. Generally, the major challenge with IM delivery lies in water-soluble therapeutics, which can result in challenges in dissolution, degradation, and uptake of both the therapeutic and the formulation (109). IM injection of microspheres and hydrogels is a viable alternative to the oral delivery of certain therapeutics, thereby increasing the overall PK and dynamic profiles of the therapeutics, while decreasing the likelihood of systemic side effects $(67,111)$. Additionally, IM-administered depot injections can allow for the modulation of dosing regimens by decreasing the dosing frequency.

\subsubsection{Applications, Obstacles, and Opportunities}

Although there are drawbacks concerning patient compliance and biological responses with IM delivery, vaccine and biological therapeutic research is heavily dependent on this type of delivery $(108,109)$. As a result, a significant portion of IM 
delivery-related research is devoted to improving the sustained release and biological responses of newly developed vaccines and other biological therapeutics $(107,112)$. This interest is mainly due to the advantageous changes in PK and dynamic profiles of nanocarrier formulations, as well as the significant decrease in dosing frequency (67, 106, 111). Another novel use of nanocarriers in IM delivery is the development of adjuvants, which are entities that work to increase antigen uptake and promote dendritic cells to initiate an immune response (113). Aside from vaccine products, there are very few products using IM for the delivery of small molecules. Microspheres are the main form of technology used for sustained drug release for these applications $(106,111)$. Currently, there are no FDA-approved nanoscale IM formulations and the future of IM delivery is dependent on extending the time between doses of both small and large molecule therapeutics.

\subsubsection{Subcutaneous Delivery}

Subcutaneous (SC) administration involves the delivery of therapeutics into the interstitial area underlying the epidermis via injection. The interstitial area under the skin is primarily comprised of negatively-charged glycosaminoglycan and collagen fibers, which provides a relatively slow and steady absorption rate of molecules, owing to reduced blood flow (114). Conventional SC formulations include aqueous solutions, oily solutions, suspensions, and simple emulsions of therapeutics. In order to modify the release profile of compounds, they can be encapsulated into particle or implant-based formulations. Particle-based delivery systems can also be applied for lymph-targeting delivery (115). 
The fate of nanocarriers delivered through the SC route depends on the particle properties and the injection location (116). Particle size is the key factor in determining the final absorption route. Particles $<10 \mathrm{~nm}$ tend to be absorbed via the capillaries draining from the injection site, where they then go into systemic circulation. Particles $10-100 \mathrm{~nm}$ in diameter move through the interstitium and are taken up directly into the lymphatic system. Particles $>100 \mathrm{~nm}$ show low capability of diffusion through the water channel within the interstitium (also around $100 \mathrm{~nm}$ ). As a result, the larger particles mostly retain at the injection site and form a depot. During particle diffusion through interstitium, charge and hydrophilicity play important roles as they influence the interaction between particles and interstitial compounds. Furthermore, the driving force of particle transport into lymph is fluid flow from the interstitium to the lymphatics, which varies in different locations. Correspondingly, the variation of injection site also affects the lymphatic transport by changing the fluid flow and pressure surrounding the formulation (116-119).

\subsubsection{Applications and Advantages}

SC administration of therapeutics is widely applied to obtain higher bioavailability, to modify the release profile to allow for longer systemic exposure, or to serve as an alternative dosing route when oral delivery is not applicable (115). Various nanocarrier-based SC formulations have been developed to deliver therapeutics such anti-cancer drugs (118), antibiotics (106), HIV drugs (120), peptide

hormones (121), anticoagulants (122), protein drugs (123), mRNA (124), and immunosuppressive anti-rheumatic drugs (125). Such nanocarrier-based formulations 
have been developed for the treatment of pancreatic, non-small cell lung, breast, ovarian cancers (118), gastrointestinal clostridium difficile-associated diseases (106), tuberculosis (126), lymphatic filariasis (116), venous thrombosis (122), inflammatory diseases (123), rheumatoid arthritis (125), and the prevention of HIV-1 vaginal transmission (1). The function of these particular nanocarriers can be divided into three aspects, including 1) the formation of depots at the injection site in order to provide sustained release of therapeutics $(106,118,120-122,125,126), 2)$ the application of particles $70-110 \mathrm{~nm}$ in diameter for lymphatic system targeting (116, 125), or 3) systemic delivery of nanocarriers via the SC route (124).

Compared to other parenteral routes such as IV and IM administration, SC administration is typically convenient to patients. First, it is often less painful and less time consuming, which improves patient compliance. Furthermore, it is easy to do and suitable for self-administration, which reduces the total cost of treatment $(119,127)$. In addition, nanocarrier formulations delivered via the SC route provide protection for the therapeutic and result in prolonged release, thereby reducing dosing frequency, improved safety and efficiency, and targeted delivery of therapeutics (116).

\subsection{Pulmonary Delivery}

In recent years, pulmonary delivery has been a growing interest owing to its advantages over other delivery systems to treat local diseases in the lungs and respiratory tract such as asthma, infectious diseases, cancer, and chronic obstructive pulmonary diseases (COPD) (128). Local administration to the lungs provides high concentration of a drug at the site of action, lowering the overall dose required, 
thereby reducing systemic side effects $(129,130)$. Due to the high surface area in the lungs (75-140 $\mathrm{m}^{2}$ in total), systemic delivery can be attained via the alveoli, where the drug can be absorbed into the bloodstream (131-133). Absorption by the alveoli allows for a rapid onset of action and avoidance of first-pass metabolism $(134,135)$.

\subsubsection{Delivery Devices}

The three major types of inhalers used for pulmonary delivery are metered dose inhalers (MDIs), nebulizers, and dry powder inhalers (DPIs) (136, 137). The mechanisms of delivery and the formulation requirements differ by the type of inhaler used. MDIs are pressurized vessels that rely on the driving force of the propellant to atomize droplets containing $\operatorname{drug}(\mathrm{s})$ and excipients, where the drugs are either solutions or particulate suspensions $(136,138)$. Nebulizers deliver liquid drug formulations in a steady stream of droplets of 1-5 $\mu \mathrm{m}$, and can deliver larger doses compared to other inhalers, but may also require longer dosage times (139). Nebulizers produce ultra-fine droplets with high polydispersity, which can make them unsuitable for the delivery of larger particles (bigger than the droplet size) (131). Dry powder inhalers (DPIs) are breath-activated devices that deliver therapeutics in the form of a dry powder directly to the airways. During administration, a capsule containing a set dose of drug is punctured and the drug is aerosolized (140). Therapeutics delivered using DPIs tend to agglomerate due to hygroscopic phenomena and electrostatic interactions, which reduce aerosolization performance (131). DPIs can be preferred over other devices owing to the promise of physical stability of the 
formulation in solid form, ease to use and transportation, and application to variety of active ingredients and dose weights $(141,142)$.

\subsubsection{Particle Fate}

Particle deposition in the lungs is governed by the aerodynamic diameter of the particles and occurs by one of three mechanisms: inertial impaction, sedimentation, or diffusion. Additionally, inhalable particles can be categorized into different sizes, including coarse $(\geq 5 \mu \mathrm{m})$, fine $(0.5-5 \mu \mathrm{m})$, or ultrafine $(\leq 0.5 \mu \mathrm{m})$ (133). Coarse carriers collide with the upper airways (trachea and large bronchi) and deposit by impaction, fine particles can reach the lower lung airways by sedimentation, and ultrafine particles can deposit throughout the respiratory tract, reaching the alveoli by diffusion $(132,133)$. Particles within the fine and ultrafine categories fall in the $1-5$ $\mu \mathrm{m}$ range of values for deposition by sedimentation (45).

\subsubsection{Microparticles}

Inhalable microparticles (MP) can be categorized as coarse or fine particles. While MP are exceptionally larger than nanoparticles (NP), properly engineered MP are able to reach deeper regions of the lungs $(45,143)$. In most cases, particles in the micron range deposit via impaction or sedimentation (133). While spherical particles are the most recognized systems, porous and corrugate particles have shown exceptional results and promise for pulmonary administration (144). Although porous MP are inherently large in geometric size (coarse size range), the aerodynamic diameters are low, while the internal pores allow for low mass density and high 
surface area $(144,145)$. The characteristics allow for the avoidance of macrophage uptake, while achieving favorable aerodynamic and drug release performances (49). Corrugated morphology allows for deeper lung delivery in comparison to spherical morphology particles. This is likely linked to the inherent decreases in particle cohesion and smaller aerodynamic diameter, as a result of the corrugations $(145,146)$.

\subsubsection{Nanoparticles}

Nanoparticles (NP) fall within the range of fine or ultrafine inhalable particles. Through utilization of their size, NP are able to deposit in the lower areas of the lungs, via Brownian diffusion, and are able to reach the alveoli (133). Additionally, NP offer

an increase in surface area to volume ratio, which enhances the solubility and dissolution rate of poorly water-soluble drugs. Deposition into the alveoli as well as the increased dissolution rate promotes increased absorption, allowing for increases in overall bioavailability $(130,137,147)$. However, it should be noted that these particles, due to their size and density, are likely to be exhaled (133). To bypass this challenge, Nebulizers, MDI, and DPI can be used to generate aerosols (131, 133, 139, $141,142)$. Another method to overcome this challenge is through the design of nanocomposite microparticle (nCmP) systems. By forming aggregates of $\mathrm{NP}, \mathrm{nCmP}$ are able to utilize the advantages presented with corrugated MP structures (44). As NP aggregate, they form a MP-like structure, increasing the inherent size of the system. Upon initial inhalation, $\mathrm{nCmP}$ are able to stay in their MP-like structure. Once $\mathrm{nCmP}$ reach fluid in the lungs, they dissociate to NP and deposit further into the lungs (54). 


\subsubsection{Advantages and Applications}

Oral and IV administration are the two most common methods of therapeutic delivery (1). However, due to systemic exposure, therapeutics that demonstrate a small therapeutic window and/or are potent are likely to be associated with side effects and/or toxicity. Administration of particle-based delivery systems via inhalation can limit systemic exposure, thereby decreasing overall side effects (130). Pulmonary administration allows for localized delivery of therapeutics. For example, diseases such as tuberculosis and lung cancer are treated with potent drugs via extended dosing regimens, often via IV administration (148-150). Localized administration can decrease such regimens while altering the administration route, thereby increasing overall patient compliance (148). Complimenting this is the potential for increased treatment efficacy, as localized delivery can promote increased drug exposure to sites of interest. The other major advantage of pulmonary administration is the potential for increased bioavailability via increased absorption and bypassing of first-pass metabolism $(44,130,147)$. The combination of a thin epithelial monolayer, high vascularization, and high surface area of the alveoli can allow administered therapeutics to diffuse into the bloodstream, promoting high absorption rates and rapid onsets of action (133). Additionally, the lungs have minimal enzymatic activity, limiting the amount of drug metabolism that occurs (130).

The advantages provided by pulmonary administration promote improved treatment options for diseases such as cystic fibrosis, lung cancer, pulmonary hypertension, and other disease states that can have severe impacts on both the pulmonary system, and the patient $(129,151,152)$. Additionally, due to the limited 
enzymatic activity in the lungs, delivery of macromolecules via inhalation is another major research interest (130). Bypassing IV administration via pulmonary administration for macromolecule agents (vaccines, peptides, etc.) can greatly increase patient compliance of such entities (145). An example of a successful pulmonary drug delivery system is $\mathrm{TOBI}^{\circledR}$ Podhaler ${ }^{\mathrm{TM}}$, a dry powder dosage form of tobramycin developed for the treatment of Pseudomonas aeruginosa infections in cystic fibrosis patients $(60,153)$.

\subsubsection{Challenges}

Like other particle-based delivery systems, interactions with biological entities in the lungs can be an issue. Interactions among mucus, bronchial epithelium tissue, and alveolar macrophages all present significant challenges for pulmonary administered systems (129). Mucus presents a major challenge due to its viscoelastic properties and physical structure (154-156). Mucociliary clearance generally occurs in the upper airways of the lungs, where foreign material is lodged and entrapped in the mucus (154-156). Clearance then occurs when cilia transport this mixture to coordinate a coughing or swallowing response (157). Bronchial epithelium tissues, like other epithelial tissues, are inherently lipophilic with tight junctions (limiting passive paracellular transport), and contain both uptake and efflux transporters (158, 159). Although uptake transporters can promote drug absorption, efflux transporters

will demote it. Alveolar macrophages in the lower airways are capable of phagocytosing foreign materials, including particle-based delivery systems that are approximately a few micron or less in diameter (160). A diagram of potential 
biological barriers can be seen in Figure 2.1B. To avoid these major barriers, particlebased delivery systems need to be carefully designed and tailored to bypassing specific challenges.

\subsubsection{Future Opportunities}

Present challenges and the growth of biological therapeutics have both created continual opportunities for pulmonary administration of particle-based delivery systems. It is important to note that fundamental characteristics including particle size, morphology, and surface charge can also provide options to bypass challenges faced in pulmonary delivery $(133,145,161,162)$. The importance of size is highlighted in sections 2.3.2.1 and 2.3.2.2. Surface morphology plays a significant role in the deposition of particles, particularly for MPs, and is highlighted in section 2.3.2.1. In terms of surface charge, neutrally charged systems show the highest promise for pulmonary administration $(54,163)$. While cationic surfaces can increase cell toxicity, and negative surfaces can increase alveolar macrophage uptake, functionally neutral surfaces have shown exception promise in avoiding macrophage uptake, as well as increased mucus diffusion (163-166).

A major research interest for pulmonary delivery systems is the particle engineering of systems that can readily diffuse through mucus for the treatment of multiple disease states (167-170). Bypassing of the mucus to increase the treatment of bacterial diseases, especially those associated with cystic fibrosis stands as an exceptionally interesting research topic currently $(155,171,172)$. Another research interest is the understanding of transport phenomena across epithelial tissue. Although 
a newer point of interest, results from numerous studies show that particle systems, particularly NP, utilize a multitude of diffusion methods to cross epithelial tissue, warranting more research to be devoted to understanding these phenomena and possible explanations (6). 


\title{
CHAPTER 3
}

\section{ENHANCEMENT OF MACROPHAGE UPTAKE VIA PHOSPHATIDYLSERINE- COATED ACETALATED DEXTRAN NANOPARTICLES}

Published in Journal of Drug Delivery Science and Technology

Volume 50, pages 57-65, 2019

\author{
Nishan K. Shah ${ }^{1}$, Sweta K. Gupta ${ }^{2}$, Zimeng Wang ${ }^{2}$, Samantha A. Meenach ${ }^{1,2, *}$ \\ ${ }^{1}$ University of Rhode Island, College of Pharmacy, Department of Biomedical and \\ Pharmaceutical Sciences, Kingston, RI 02881 \\ ${ }^{2}$ University of Rhode Island, College of Engineering, Department of Chemical \\ Engineering, Kingston, RI 02881
}




\subsection{Abstract}

Although vital to the body's immune system, macrophages can also act as reservoirs for pathogens from infectious diseases such as tuberculosis and the human immunodeficiency virus. Limitations in the treatment of such diseases include the challenge of targeting therapeutics directly to the macrophages and the large systemic dosages often needed for treatment. The objective of the present study is to develop a nanoparticle (NP)-based drug delivery system that can more effectively deliver therapeutic agents into macrophages. Acetalated dextran (Ac-Dex) NP loaded with the lipophilic model compound curcumin (CUR) were synthesized and coated in 1,2dipalmitoyl-sn-glycero-3-phospho-L-serine (DPPS), a phospholipid that induces phagocytosis in macrophages. DPPS-CUR NP were found to release $67.8 \%$ of encapsulated CUR within 24 hours at $\mathrm{pH} 5.35$ and exhibited minimal CUR release (6.3\%) at $\mathrm{pH}$ 7.4. DPPS-CUR NP were uptaken by murine macrophages significantly more than NP without DPPS coating and NP exposure to these macrophages resulted in minimal toxicity to the cells and minimal nitric oxide production. These results suggest that the combination of the DPPS coating and $\mathrm{pH}$-sensitive polymer Ac-Dex can provide a NP delivery system capable of enhanced uptake by macrophages and potential systemic stability to more effectively deliver drugs of interest. As a result, the described DPPS-CUR NP can serve as a viable delivery system for the treatment of macrophage-associated diseases. 


\subsection{Keywords}

Acetalated dextran; 1,2-dipalmitoyl-sn-glycero-3-phospho-L-serine (DPPS); macrophage-associated diseases; targeted cellular uptake; nanoparticles; drug delivery

\subsection{Abbreviations}

2-methoxypropene (2-MOP), acetalated dextran (Ac-Dex), curcumin (CUR), cyclicto-acyclic ration (CAC), dichloromethane (DCM), dimethyl sulfoxide (DMSO), deionized (DI) water, 1,2-dipalmitoyl-sn-glycero-3-phosphocholine (DPPC), 1,2dipalmitoyl-sn-glycero-3-phospho(1'-rac'glycerol) (DPPG), 1,2-dipalmitoyl-snglycero-3-phospho-L-serine (DPPS), differential scanning calorimetry (DSC), encapsulation efficiency (EE), human immunodeficiency virus (HIV), lipopolysaccharide (LPS), nitric oxide (NO), nanoparticles (NP), phosphate buffered saline (PBS), phosphatidylserine (PS), poly(vinyl alcohol) (PVA), poly(lactic-coglycolic acid) (PLGA), p-toluenesulfonate (PPTS), scanning electron microscopy (SEM), tuberculosis (TB), transmission electron microscopy (TEM), triethylamine (TEA) 


\subsection{Introduction}

Macrophages are vital in the removal of cellular debris and foreign bodies to maintain homeostasis in the human body $(82,173)$, can colonize in the liver, lungs, spleen, lymph nodes, marrow, or brain, and are critical to the innate immune system (173). An example of the importance of macrophages is in the removal of apoptotic cells via stimulation of various signals and markers that are overexpressed by presenting cells to initiate identification and engulfment (174). Although macrophages are involved in the protection and maintenance of the human body, there are scenarios in which these cells can result in more harm than good. Macrophages have the potential to act as reservoirs for infectious pathogens, including those related to two of the most prevalent infectious diseases, tuberculosis (TB) $(82,173)$ and the human immunodeficiency virus (HIV) $(82,173)$. The commonality amongst these diseases lies in the extensive treatment times and dosing regimens necessary to treat $\mathrm{TB}$ and HIV, often resulting in negative side effects. As a result, treatment by way of enhanced delivery to macrophages is of growing interest $(10,43,180-186,82,173-$ 179).

Recent progress in the development of macrophage targeting systems have been based around receptor-ligand interactions (187). One common approach is surface functionalization of systems with mannose residues to target CD206 (mannose receptor), a carbohydrate-recognition domain that is largely expressed on alveolar macrophages $(188,189)$. In other cases, tumor associated macrophages can also be targeted using peptide-based ligands such as rabies virus glycoprotein (RVG), and can be used as carriers for systems encapsulating anti-cancer therapies (190, 191). Other 
ligands such as 4-SO $\mathrm{S}_{4}$-GalNAc can be used to target other receptors on the surface of phagocytic macrophages to increase targeted uptake (192).

Phosphatidylserine (PS) is an anionic phospholipid that is produced and stationed on the inner membrane of healthy cells $(174,181,193,194)$. Once apoptosis is induced, PS transitions from the inner leaflet to the outer leaflet of the cell membrane (178). Apoptotic cells produce a signal via PS exposure to stimulate the attraction of macrophages for engulfment of the presenting cells (phagocytosis) via receptor-ligand interactions or identification of PS binding proteins produced by phagocytes $(82,174,194,195)$. Although it is essential for the phagocytosis signal, there is some debate as to whether sole exposure of PS is sufficient to induce the uptake of apoptotic cells by macrophages in vitro $(174,194,195)$. However, particlebased drug delivery systems that utilize PS (specifically, 1,2-dipalmitoyl-sn-glycero-3phospho-L-serine, DPPS) as the particle coating have resulted in enhanced macrophage uptake through the sole presence of PS (174), likely due to the amount of PS presented. Multiple studies centered around treatment of inflammation $(183,196)$, HIV-1 (82, 173, 180, 182), cancer (184, 185, 197), atherosclerosis (198), and MRI imaging $(10,82,175)$ have used DPPS coatings to increase the uptake of particlebased delivery systems by macrophages or have used PS as a binding target.

Acetalated dextran (Ac-Dex) is a biodegradable, biocompatible polymer comprised of the FDA-approved excipient dextran and hydrophobic acetal groups that allow for emulsion-based NP synthesis $(19,45,53)$. Unlike commonly used drug carriers such as poly(lactic-co-glycolic acid) (PLGA), the degradation kinetics of AcDex can be easily tuned by altering the ratio of cyclic to acyclic acetal groups by 
modifying the Ac-Dex synthesis reaction time $(36,40)$. The acid-sensitivity of Ac-Dex has made it a viable carrier option in applications for cancer (36, 40, 49), inflammatory-related conditions (42), vaccines (36, 40), and antibiotic delivery (54). There is a significant difference in degradation and drug release kinetics for Ac-Dex nanoparticle (NP) systems at a $\mathrm{pH}$ of 5 (faster release) versus at a pH of 7.4 (slower release) $(36,40,42,43,45,49,53,54)$. Due to this difference, Ac-Dex NP systems will exhibit slower release and stability during systemic circulation (at $\mathrm{pH}$ 7.4) until they are delivered to a site in the body with a lower $\mathrm{pH}$, such as in macrophages, where therapeutics will be readily released.

The current study involves the design of a novel therapeutic particle-based drug delivery system consisting of a phosphatidylserine (PS)-coated, polymeric (AcDex), drug-loaded NP designed to enhance uptake into macrophages. The novelty of this formulation lies in the inherent ability of PS to enhance the uptake of the NP into macrophages, while the Ac-Dex core promotes burst release of the cargo directly in the macrophages owing to its acid sensitivity. Curcumin (CUR), a natural product isolated from Curcuma longa, was chosen as the model small molecule due to its fluorescent properties, low water solubility, and potential clinical applications (43, 45, 203, 177, 181, 183, 185, 199-202).

\subsection{Materials and Methods}

\subsubsection{Materials}

Unless stated otherwise, materials were purchased from Sigma Aldrich (St. Louis, MO). 1,2-dipalmitoyl-sn-glycero-3-phosphocholine (DPPC), 1,2-dipalmitoyl- 
sn-glycero-3-phospho(1'-rac'glycerol) (DPPG), and 1,2-dipalmitoyl-sn-glycero-3phospho-L-serine (sodium salt, DPPS) were purchased from Avanti Lipids (Alabaster, $\mathrm{AL})$.

\subsubsection{Synthesis and Characterization of Acetalated Dextran}

Acetalated dextran (Ac-Dex) was synthesized using a previously described method $(45,53) .1 \mathrm{~g}$ of lyophilized dextran $(9,000-11,000 \mathrm{MW})$ was dissolved in 10 $\mathrm{mL}$ of anhydrous DMSO with $25 \mathrm{mg}$ of p-toluenesulfonate (PPTS) under nitrogen gas. The reaction was carried out for 5 minutes using $5 \mathrm{~mL}$ of 2-methoxypropene (2-MOP) and was quenched with $1 \mathrm{~mL}$ of triethylamine (TEA). The polymer was then precipitated in basic water $(\mathrm{pH} 9)$, filtered, lyophilized, and stored at $-20^{\circ} \mathrm{C}$. The cyclic-to-acyclic (CAC) ratio of Ac-Dex and acetal coverage were verified using ${ }^{1} \mathrm{H}$ NMR (Bruker $300 \mathrm{MHz}, \mathrm{NMR}, \mathrm{MA})$ as described previously $(45,53,54)$.

\subsubsection{Synthesis of PVA-Coated Nanoparticles}

PVA-coated, CUR-loaded Ac-Dex NP (PVA-CUR NP) were synthesized using a single emulsion/solvent evaporation method (53). $50 \mathrm{mg}$ of Ac-Dex and $1 \mathrm{mg}$ of CUR were dissolved in $1 \mathrm{~mL}$ of DCM. This organic solution was added to $6 \mathrm{~mL}$ of 3\% PVA (in 1x PBS) and the resulting mixture was emulsified using a probe sonicator attached to an ultrasonic processer at $120 \mathrm{~W}$ and $20 \mathrm{kHz}$ (Q500, Qsonica, Newtown, CT) for 1 minute with 1 second on/off pulses. The emulsion was added to $40 \mathrm{~mL}$ of 0.3\% PVA and was spun for 4 hours to allow for organic solvent evaporation and particle hardening (45). The spinning solution was centrifuged at $3124 \times \mathrm{x}$ for 60 
minutes and particles were redispersed in a 0.1\% PVA solution and frozen overnight, followed by lyophilization. PVA-coated NP without CUR (PVA-Blank NP) were formulated similarly to PVA-CUR NP by omitting CUR from the organic solutions. NP samples were stored at $-20^{\circ} \mathrm{C}$.

\subsubsection{Synthesis of DPPS and DPPC-Coated Ac-Dex NP}

PVA-coated NP were coated with DPPS via a film hydration method often used to make liposomal delivery systems $(180,182,183,196,204,205)$. DPPS was dissolved in $4 \mathrm{~mL}$ of chloroform and methanol $(9: 1 \mathrm{v} / \mathrm{v})(185)$. The solution was subjected to rotary evaporation using a Heidolph 2 rotary evaporator (Schwabach, Germany) to create a thin film in a $25 \mathrm{~mL}$ round-bottom flask. The film was dried under vacuum for 1 hour to remove excess solvent, during which the NP were washed with deionized (DI) water to remove excess PVA. The dried film containing the NP was then rehydrated with $4 \mathrm{~mL}$ of DI water and this solution was sonicated for 30 minutes, followed by freezing and lyophilization. After lyophilization, the resulting NP were stored at $-20^{\circ} \mathrm{C}$. The ratio of lipid to NP during the process was $1: 5(w / w)$. NP coated with DPPC and a small amount of DPPG NP were synthesized using the same method with a 5:1 (w/w) DPPC:DPPG ratio and these NP are referred to as DPPC-NP.

\subsubsection{Nanoparticle Size and Surface Charge Characterization}

Hydrodynamic diameter and surface charge of the NP were evaluated via dynamic light scattering (DLS) and zeta potential analysis, respectively, using a 
Malvern Nano Zetasizer (Malvern Instruments, Worcestershire, UK). The NP were diluted to $0.25 \mathrm{mg} / \mathrm{mL}$ in DI water and were analyzed at $25^{\circ} \mathrm{C}$ and an angle of $90^{\circ}$.

\subsubsection{Evaluation of CUR NP Encapsulation Efficiency and Drug Loading}

To determine the amount of CUR encapsulated in the NP systems, NP were dissolved in DMSO (1 mg/mL) and the fluorescence of the solutions was analyzed using a SpectraMax M2 Plate Reader (Molecular Devices, Sunnyville, CA) at an excitation of $420 \mathrm{~nm}$ and emission of $520 \mathrm{~nm}$. The encapsulation efficiency (EE) and drug loading were calculated using the following equations:

$$
\text { Encapsulation Efficiency }(E E)=\frac{\text { experimental mass of drug in } N P}{\text { theoretical mass of drug in } N P} \times 100 \%
$$

$$
\text { Drug Loading }=\frac{\text { mass of drug in } N P}{\text { mass of } N P}
$$

\subsubsection{Electron Microscopy Imaging Analysis of Nanoparticles}

Images of the NP were taken using a Zeiss Sigma VP Field Emission-Scanning Electron Microscope (FE-SEM) (Germany) for analysis of NP morphology. After NP were suspended in basic water $(15 \mathrm{mg} / \mathrm{mL}), 1-2$ drops of this suspension were added to aluminum SEM stubs (TedPella Inc., Redding, CA), and the samples were air dried. Dried samples were then coated with a film of gold/palladium alloy using an Emscope SC400 sputter coating system at a $20 \mu \mathrm{A}$ for 75 seconds under argon. Images of the NP systems were also captured via a JEOL JEM-2100F transmission electron 
microscope (TEM, Peabody, MA) for observation of the NP core and outer layers. 1 $\mu \mathrm{L}$ of $15 \mathrm{mg} / \mathrm{mL} \mathrm{NP}$ suspension in water were placed on 200 square mesh copper grids (Election Microscopy Sciences, Hatfield, PA) and air dried prior to imaging.

\subsubsection{Differential Scanning Calorimetry (DSC) Analysis of Nanoparticles}

Thermal phase transitions of the NP systems were analyzed using differential scanning calorimetry (DSC) via a TA Q10 DSC system (TA Instruments, New Castle, DE) connected to an RSC-90 cooling accessory. For dry-state DSC, samples were lyophilized 24 hours prior to analysis. $1-3 \mathrm{mg}$ of $\mathrm{NP}$ were analyzed at $10^{\circ} \mathrm{C} / \mathrm{min}$ from 0 to $300^{\circ} \mathrm{C}$. For wet state analysis, NP were dispersed in DI water $(0.1 \mathrm{mM} \mathrm{DPPS})$ and $15 \mu \mathrm{L}$ of the NP suspension was added to aluminum pans to be analyzed at $2^{\circ} \mathrm{C} / \mathrm{min}$ and from 5 to $65^{\circ} \mathrm{C}$. DSC analysis of the raw materials was also performed.

\subsubsection{PVA Coating Quantification}

Quantification of the amount of PVA on the surface of NP was completed using a previously described method (206). NP samples were dispersed in DI water (1 $\mathrm{mg} / \mathrm{mL}) .400 \mu \mathrm{L}$ of sample solution, $300 \mu \mathrm{L}$ of iodine solution (1.25\% iodine and $2.5 \%$ potassium iodide), and $1.5 \mathrm{~mL}$ of $4 \%$ boric acid solution were mixed together for 20 minutes at room temperature and $100 \mathrm{rpm}$. Absorbance of the samples was measured at a wavelength of $630 \mathrm{~nm}$. 


\subsubsection{Phospholipid Content Quantification}

Phospholipid quantification was carried out using Stewart's method (207). Briefly, $2.7 \mathrm{~g}$ ferric chloride hexahydrate and $3 \mathrm{~g}$ ammonium thiocyanate were dissolved in $100 \mathrm{~mL}$ of distilled water. NP samples were dissolved in chloroform (1 $\mathrm{mg} / \mathrm{mL}$ ) and were mixed with the ammonium ferrothiocyanate solution via vortexing for 5 minutes at a 2:1 (v/v) ratio. After mixing, the solution was allowed to separate, and the phospholipid content in the chloroform portion was measured via UV-vis spectroscopy at $488 \mathrm{~nm}(\mathrm{DPPC} / \mathrm{G})$ and $452 \mathrm{~nm}$ (DPPS).

\subsubsection{In Vitro Drug Release from CUR NP}

Release of CUR from the NP was carried out using a previously established centrifugation technique (45). $1 \mathrm{mg} / \mathrm{mL} \mathrm{NP}$ samples were dispersed in PBS (pH 7.4) or sodium acetate buffer (pH 5.35) supplemented using $0.2 \%$ Tween ${ }^{\circledR} 80$ to enhance CUR solubility. The suspensions were incubated at $37^{\circ} \mathrm{C}$ and $100 \mathrm{rpm}$ and at predetermined time points the suspensions were removed and centrifuged at 23,102 $\mathrm{x}$ $g$ for 15 minutes. $200 \mu \mathrm{L}$ of the supernatant was collected and frozen at $-20^{\circ} \mathrm{C}$ and 200 $\mu \mathrm{L}$ of fresh medium was added to the particle solution prior to re-dispersion and reincubation. Release samples were mixed with DMSO (1:1 by volume) prior to fluorescence spectroscopy analysis at an excitation of $420 \mathrm{~nm}$ and emission of $520 \mathrm{~nm}$ (45). 


\subsubsection{Cell Culture}

Murine macrophage cells (RAW 264.7) and A549 human adenocarcinoma cells obtained from American Type Culture Collection (ATCC, Manassas, VA) were used for cell culture studies. The cells were maintained at $37^{\circ} \mathrm{C}$ and $5 \% \mathrm{CO}_{2}$ in Dulbecco's modified Eagle's medium (DMEM) supplemented with $10 \%$ (v/v) fetal bovine serum, $100 \mathrm{U} / \mathrm{ml}$ penicillin, $100 \mu \mathrm{g} / \mathrm{ml}$ streptomycin, Fungizone ${ }^{\circledR}(0.5 \mu \mathrm{g}$ amphotericin $\mathrm{B}, 0.41 \mu \mathrm{g} / \mathrm{mL}$ sodium deoxycholate), and $1 \mathrm{mM}$ sodium pyruvate.

\subsubsection{In Vitro Cytotoxicity Analysis of NP}

The cytotoxic effect of the formulated NP on RAW 264.7 macrophages was determined using a resazurin assay. Cells were seeded in 96-well plate at 5,000 cells/well and incubated overnight at $37^{\circ} \mathrm{C}$ and were then exposed to varying concentrations of PVA-CUR and DPPS-CUR NP (0.001 to $0.2 \mathrm{mg} / \mathrm{ml})$. Untreated cells were used as negative controls. After 48 hours, resazurin solution $(60 \mu \mathrm{M})$ was added to the cells and incubated for 3 hours. The fluorescence intensity of resorufin produced by viable cells was detected at $544 \mathrm{~nm}$ (excitation) and $590 \mathrm{~nm}$ (emission) using BioTek Cytation 3 plate reader. The relative viability of each sample was calculated by:

$$
\text { Relative Viability }=\frac{\text { Sample Fluorescence Intensity }}{\text { Control Fluorescence Intensity }} \times 100
$$




\subsubsection{Nitrite Analysis}

A Griess assay was performed to determine nitric oxide (NO) production by macrophages. Following 48-hour incubation with varying concentrations of NP (CURloaded and blank), 96-well plates containing the samples were centrifuged and $50 \mu \mathrm{L}$ of the resulting supernatant was removed from each well. Griess reagents were added per manufacturer's instructions and the absorbance was measured at $550 \mathrm{~nm}$. LPS (500 ng/mL), was used to promote NO production as a control.

\subsubsection{In Vitro Cellular Uptake Via Spectroscopy and Confocal Microscopy}

Cellular uptake of CUR-loaded NP by RAW 264.7 and A549 cells was observed using a Cytation 3 image reader (BioTek, Winooski, VT). Cells (7,500 cells/well) were seeded in a 96-well plate and incubated overnight at $37^{\circ} \mathrm{C}$. Cells were then incubated with $0.1 \mathrm{mg} / \mathrm{ml}$ of NP solutions and equal concentration of CUR for 1 and 3 hours. After incubation, the media was removed, and the cells were washed three times with $200 \mathrm{mM}$ glycine to remove any unbound NP that were not taken up by the cells. For quantification of cellular uptake, the fluorescence of the CUR within the cells was analyzed via fluorescence spectroscopy at $420 \mathrm{~nm}$ (excitation) and 520 $\mathrm{nm}$ (emission).

RAW 264.7 and A549 cells were seeded into $35 \mathrm{~mm}$ glass-bottom petri dishes at a concentration of 500,000 cells per dish and allowed to grow overnight. DPPSCUR NP, DPPC-CUR NP, or PVA-CUR NP were suspended and bath sonicated in cell culture medium at $0.1 \mathrm{mg} / \mathrm{mL}$ and were then incubated with cells for 1 or 3 hours. Cells were washed 3x in PBS and incubated in fresh media containing CellMask Deep 
Red (Invitrogen) at $1 \mu \mathrm{L} / \mathrm{mL}$ media for 10 minutes. Cells were then fixed with $4 \%$ paraformaldehyde for 10 minutes and rinsed 3x with PBS. Fresh PBS was added and cells were immediately imaged using a Nikon Eclipse Ti2 inverted confocal fluorescent microscope.

\subsubsection{Statistical Analysis}

All measurements were performed in at least triplicate. Statistical differences for in vitro cellular studies was determined using one-way or two-way ANOVA with Tukey's multiple comparisons test (GraphPad Prism version 7). For cumulative drug release, Student's $t$-Test was used to determine statistical differences. A p-value of $<$ 0.05 or lower was considered as statistically significant. Values are presented as mean \pm standard deviation.

\subsection{Results}

\subsubsection{Characterization of Ac-Dex Polymer and Nanoparticles}

Analysis of the acetal coverage and cyclic-to-acyclic (CAC) ratio of Ac-Dex following its synthesis was confirmed via NMR. The acetal coverage of Ac-Dex was $73 \%$, whereas the CAC ratio was $45 \%$. PVA-coated NP were synthesized using the emulsion method to create NP with an Ac-Dex polymer core and PVA coating both with and without curcumin loading (PVA-CUR NP and PVA-Blank NP, respectively). As seen in Table 3.1, PVA-coated NP were $260-275 \mathrm{~nm}$ in diameter, exhibited polydispersity index (PDI) values of 0.04 , and had nearly neutral surface charges (less than $-3 \mathrm{mV}$ ), as seen from the zeta potential values. 
After the synthesis of PVA-coated NP, the particles were coated with DPPS using a thin film hydration method to form DPPS-coated NP, both with and without CUR loading (DPPS-CUR NP and DPPS-Blank NP, respectively). The diameters for both the blank and CUR-loaded DPPS NP increased by approximately $75 \mathrm{~nm}$ in comparison to the PVA-coated NP. The PDI values were low for both DPPS NP systems (less than 0.21), however, there was a substantial decrease in zeta potential, from $-3 \mathrm{mV}$ (PVA-coated NP) to $-40 \mathrm{mV}$ (DPPS-coated NP) for both blank and CURloaded NP systems.

The encapsulation efficiency (EE) and drug loading (by mass) of CUR-loaded NP were analyzed to quantify the amount of CUR loaded in the particles. Both PVAand DPPS-coated NP exhibited EE values around 25\% whereas the drug loading was 7.1 and 5.2 $\mu \mathrm{g}$ CUR/mg NP for PVA-CUR NP and DPPS-CUR NP, respectively.

Table 3.1. Diameter, polydispersity index (PDI), surface charge via zeta potential, curcumin encapsulation efficiency, and drug loading of nanoparticle system (mean \pm standard deviation, $\mathrm{n}=3$ ).

\begin{tabular}{|c|c|c|c|c|c|}
\hline & $\begin{array}{c}\text { Diameter } \\
(\mathbf{n m})\end{array}$ & PDI & $\begin{array}{c}\text { Zeta } \\
\text { Potential } \\
(\mathbf{m V})\end{array}$ & $\begin{array}{c}\text { Drug } \\
\text { Encapsulation } \\
\text { Efficiency }(\%)\end{array}$ & $\begin{array}{c}\text { loading } \\
\boldsymbol{( \mu g} \\
\mathbf{d r u g} / \mathbf{m g} \\
\mathbf{p a r t i c l e})\end{array}$ \\
\hline $\begin{array}{c}\text { PVA- } \\
\text { Blank NP }\end{array}$ & $262.6 \pm 1.9$ & $\begin{array}{c}0.04 \pm \\
0.00\end{array}$ & $-2.2 \pm 3.5$ & --- & -- \\
\hline $\begin{array}{c}\text { PVA- } \\
\text { CUR NP }\end{array}$ & $272.6 \pm 10.4$ & $\begin{array}{c}0.04 \pm \\
0.01\end{array}$ & $-3.0 \pm 0.6$ & $25.1 \pm 0.1$ & $7.1 \pm 1.8$ \\
\hline $\begin{array}{c}\text { DPPS- } \\
\text { Blank NP }\end{array}$ & $335.6 \pm 3.8$ & $\begin{array}{c}0.21 \pm \\
0.43\end{array}$ & $-40.4 \pm 3.3$ & --- & -- \\
\hline $\begin{array}{c}\text { DPPS- } \\
\text { CUR NP }\end{array}$ & $350.5 \pm 16.9$ & $\begin{array}{c}0.13 \pm \\
0.65\end{array}$ & $-40.6 \pm 1.3$ & $24.5 \pm 0.1$ & $5.2 \pm 0.7$ \\
\hline
\end{tabular}




\subsubsection{Morphological Analysis of NP Via Electron Microscopy}

Scanning electron microscopy (SEM) and transmission electron microscopy (TEM) were used to analyze the morphology and structure of PVA- and DPPS-coated NP loaded with CUR. SEM micrographs indicated that PVA-CUR NP presented spherical, smooth morphology and were homogenous with respect to in size (Figure 3.1, top). TEM images allowed for the visualization of a thin layer around the Ac-Dex polymer core, which likely corresponds to the PVA coating. DPPS-CUR NP were fairly monodisperse with spherical, smooth morphology as seen via SEM imaging

(Figure 3.1, bottom). TEM micrographs show a coating on the Ac-Dex NP core, likely indicating the DPPS coating on the NP.

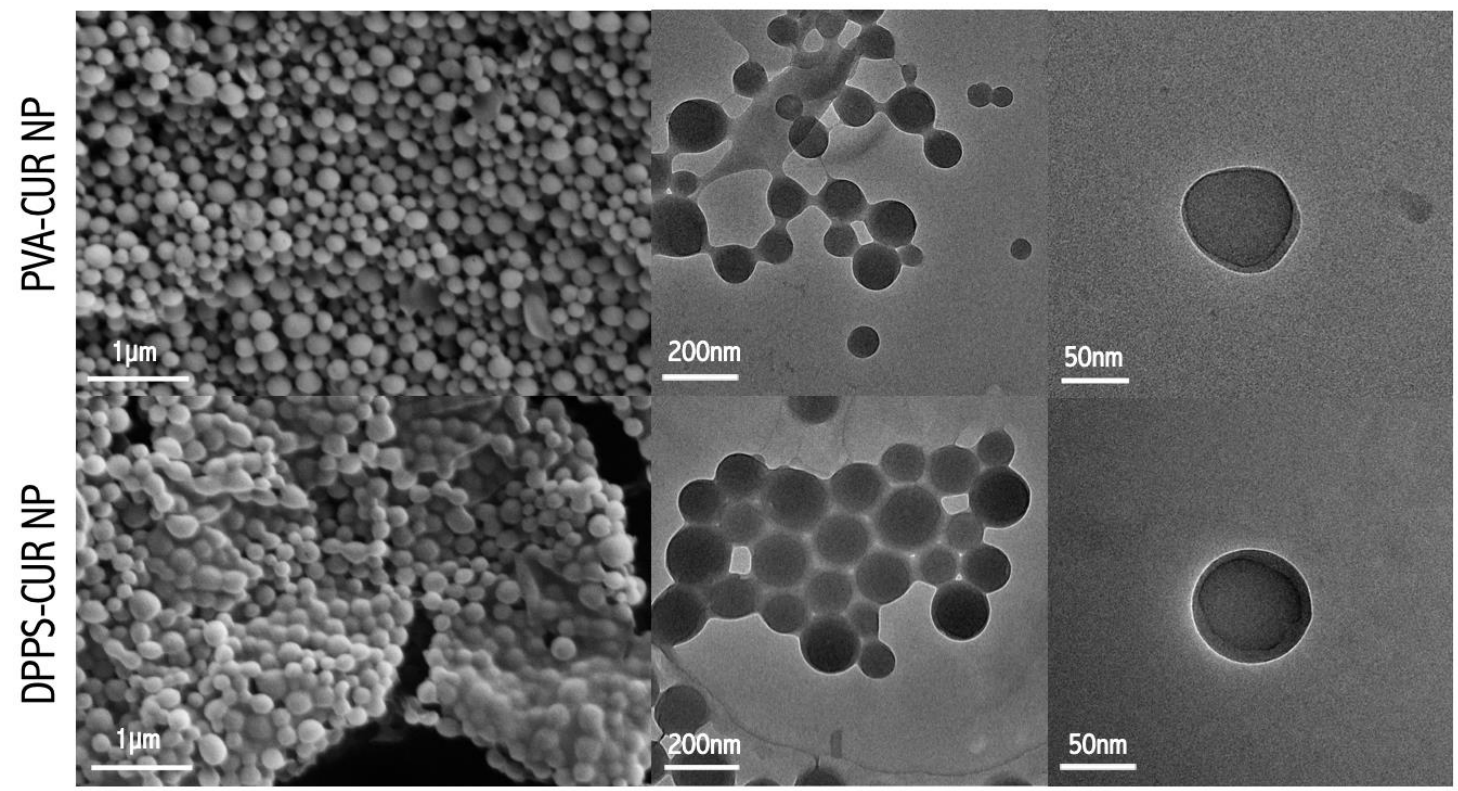

Figure 3.1. Representative SEM (left) and TEM (middle, right) micrographs of PVAand DPPS-coated, CUR-loaded nanoparticles (PVA-CUR NP and PVA-DPPS NP, respectively). 


\subsubsection{Coating Quantification}

The amount of PVA and total phospholipids present in the NP formulations were quantified and are presented in Figure 3.2. There was significantly less PVA present on the DPPS and DPPC-NP in comparison to the PVA-NP ( $p<0.001$ and $p<$ 0.0001, respectively). In addition, significantly more phospholipid was present in DPPS and DPPC-NP in comparison to PVA-NP ( $p<0.01)$.

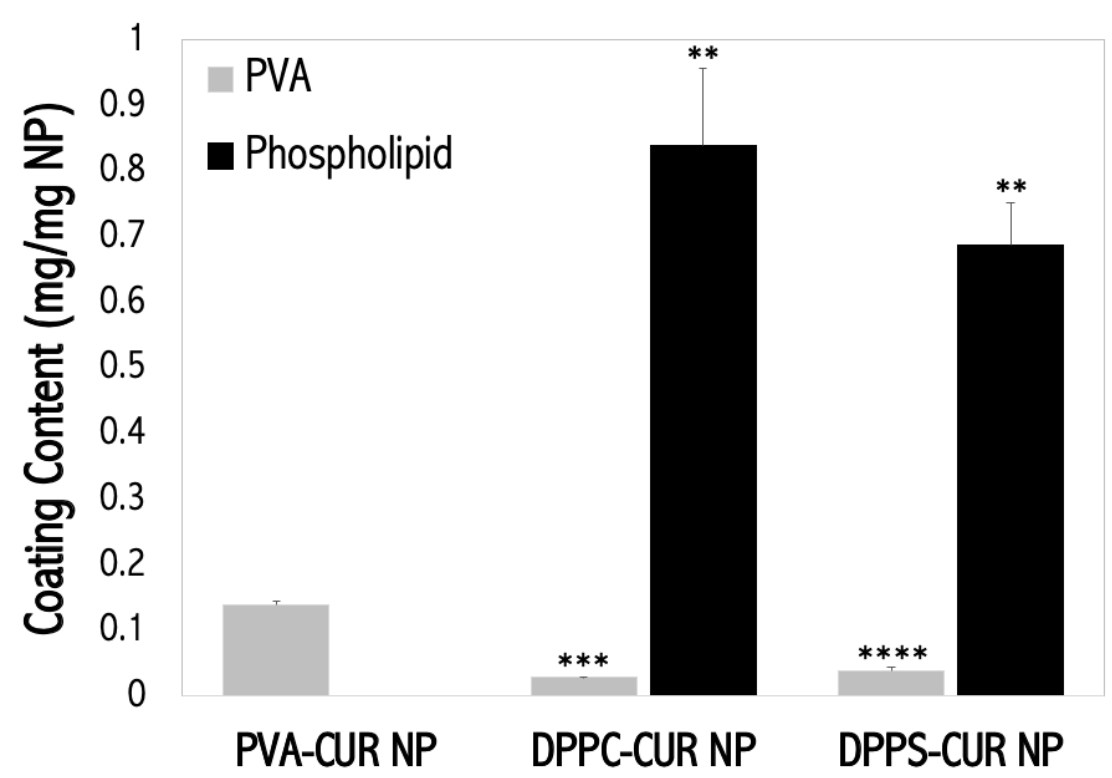

Figure 3.2. Quantification of the amount of poly(vinyl alcohol) (PVA) and total phospholipid in PVA-CUR, DPPC-CUR, and DPPS-CUR nanoparticles (NP) $*^{*} \mathrm{p}<$ $0.05, * * \mathrm{p}<0.01, * * * \mathrm{p}<0.001$ in comparison to PVA-CUR NP).

\subsubsection{Differential Scanning Calorimetry (DSC) Thermal Analysis}

Figure 3.3 shows the thermograms of the prepared NP and their corresponding raw components in their dry states. An endothermic peak at $90^{\circ} \mathrm{C}$ was present, 
indicating a bilayer phase transition for the DPPS-coated NP, which is slightly lower than the endothermic phase transition temperature of raw DPPS at $99^{\circ} \mathrm{C}$. An endothermic peak was present at $187^{\circ} \mathrm{C}$ for raw PVA, whereas a broadened endothermic peak was present at $195^{\circ} \mathrm{C}$ for PVA-coated NP. Both raw CUR and AcDex exhibited endothermic peaks at $177^{\circ} \mathrm{C}$, signifying their melting points $(45,53$, 54). These prominent endothermic peaks were not present in the formulated NP. The shift from $177^{\circ} \mathrm{C}$ to a broad peak at $195^{\circ} \mathrm{C}$ for PVA-coated NP could be due to the interaction between Ac-Dex and PVA. DPPS-coated NP exhibited a broad endothermic peak at $165^{\circ} \mathrm{C}$, potentially due to the removal of the PVA and the interaction between the Ac-Dex and DPPS. For wet-state DSC analysis of the formulated NP, there were no measurable peaks present for the temperature range used; curvature corresponded to the evaporation of water. 

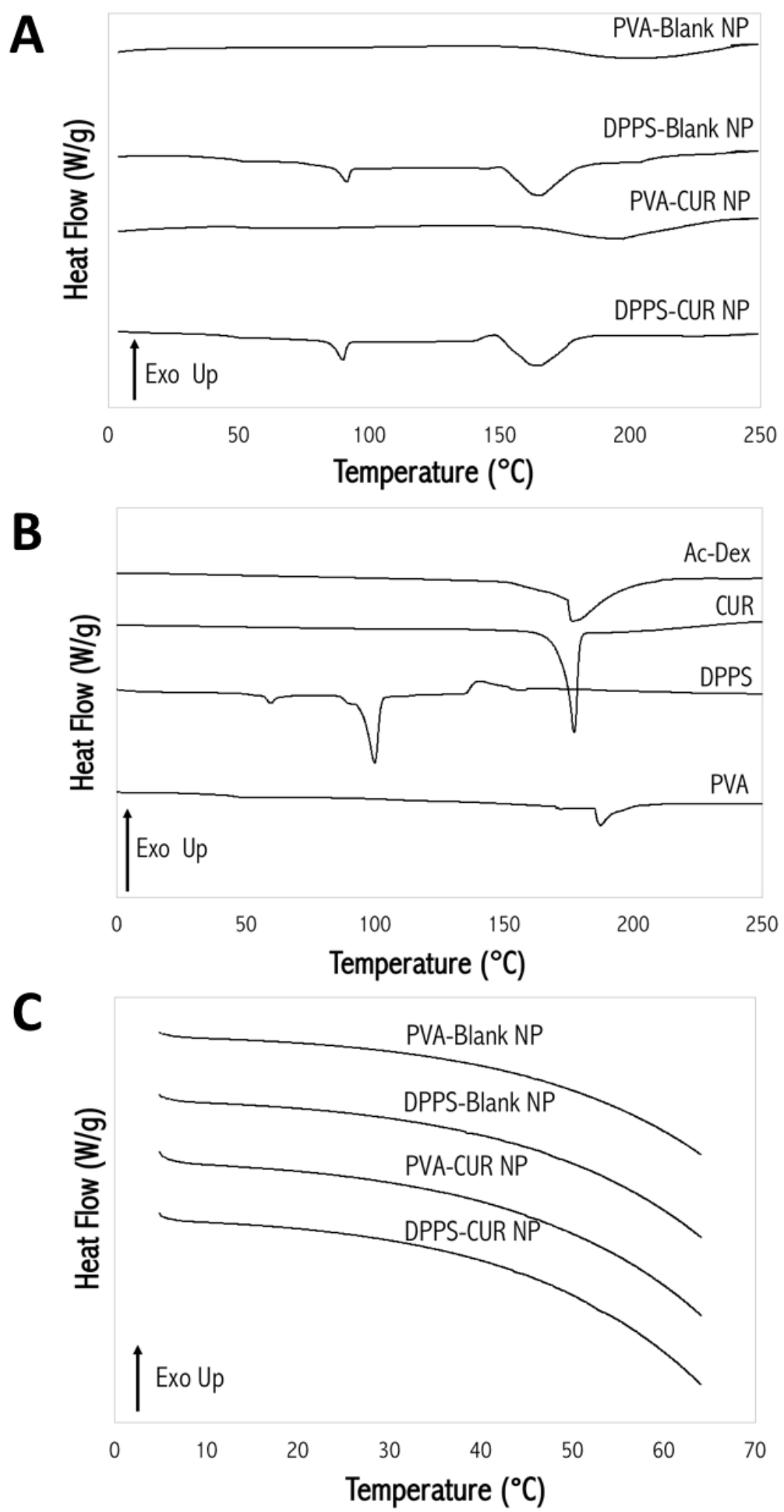

Figure 3.3. Differential scanning calorimetry (DSC) thermograms of (A) formulated nanoparticles and (B) the raw components that make up the nanoparticle systems in their dry state, and (C) formulated nanoparticles in their wet state. 


\subsubsection{In Vitro Drug Release from NP}

The release of CUR from the particles was analyzed at two $\mathrm{pH}$ values, including $\mathrm{pH} 7.4$ to simulate normal physiological $\mathrm{pH}$ and $\mathrm{pH} 5.35$ to approximate the $\mathrm{pH}$ in macrophages (43). Results in Figure 3.4 shows that there was no significant difference in the cumulative release of CUR from PVA-coated versus DPPS-coated $\mathrm{NP}$ at $\mathrm{pH} 7.4$ after 24 hours (5.2\% vs 6.3\%), signifying that the NP coating played no appreciable role in affecting CUR release at this $\mathrm{pH}$. However, significantly more CUR was released at pH 5.35 for both DPPS- and PVA-coated particles $(67.8 \%$ and $88.8 \%$ release at $\mathrm{pH} 5.35$, respectively) in comparison to $\mathrm{pH} 7.4(\mathrm{p}<0.001)$, demonstrating the sensitivity of Ac-Dex to acidic conditions. Furthermore, when comparing DPPS- and PVA-coated NP at $\mathrm{pH} 5.35$, significantly more CUR was released from NP with a PVA coating, indicating the potential influence of the DPPS layer in delaying the release of the cargo $(\mathrm{p}<0.05)$. 


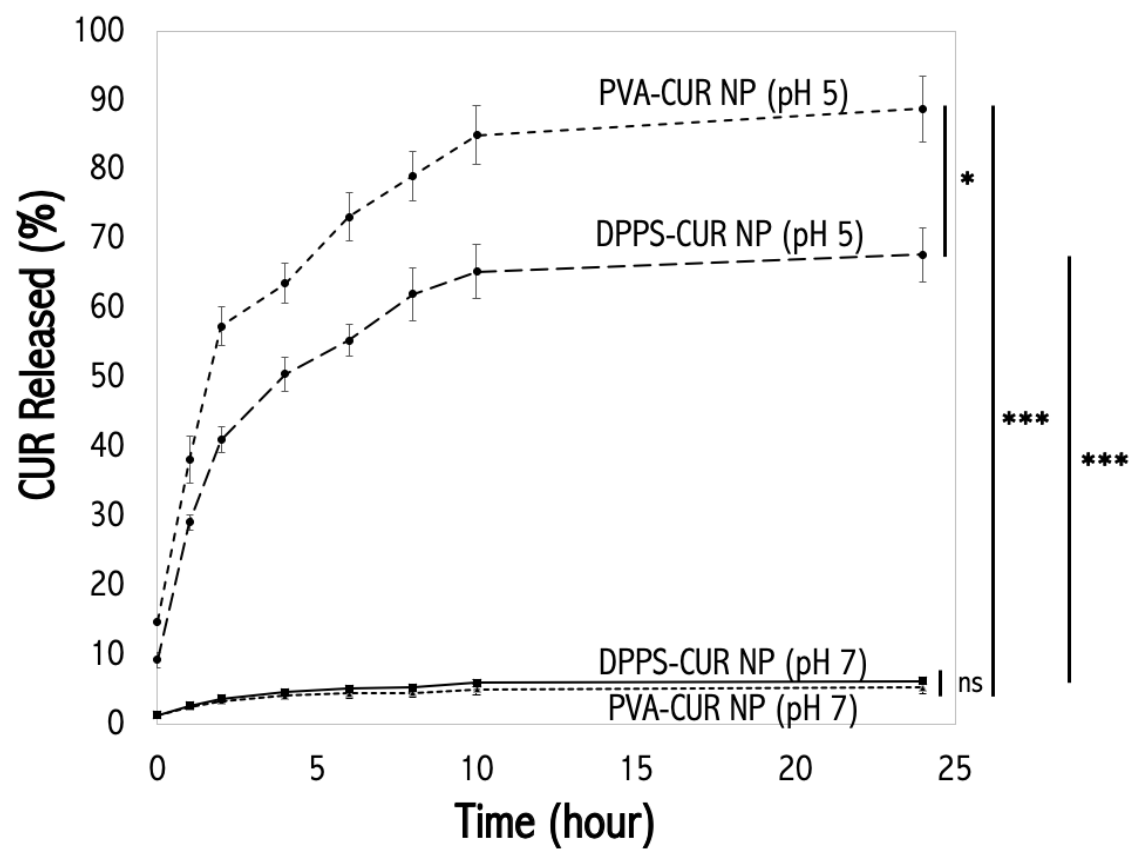

Figure 3.4. Cumulative release of curcumin (CUR) from nanoparticle systems at $\mathrm{pH} 7$ and $\mathrm{pH} 5(* \mathrm{p}<0.05, * * \mathrm{p}<0.01, * * * \mathrm{p}<0.001, \mathrm{~ns}=$ non-significant $)$.

\subsubsection{In Vitro Cytotoxicity Assay}

The impact of the described NP systems on macrophage toxicity was evaluated and the results are presented in Figure 3.5. These results indicate that the cells exhibited relative viabilities similar to the control (media only) after 48 hours of exposure to the NP systems. Increasing the concentration of NP imparted no significant change in the viability of cells for PVA-Blank NP, DPPS-Blank NP, or PVA-CUR NP for the concentrations tested. However, for DPPS-CUR NP there was a slight but statistically significant decrease in the viability of cells at $0.2 \mathrm{mg} / \mathrm{ml}$ in comparison to the control $(\mathrm{p}<0.05)$. 


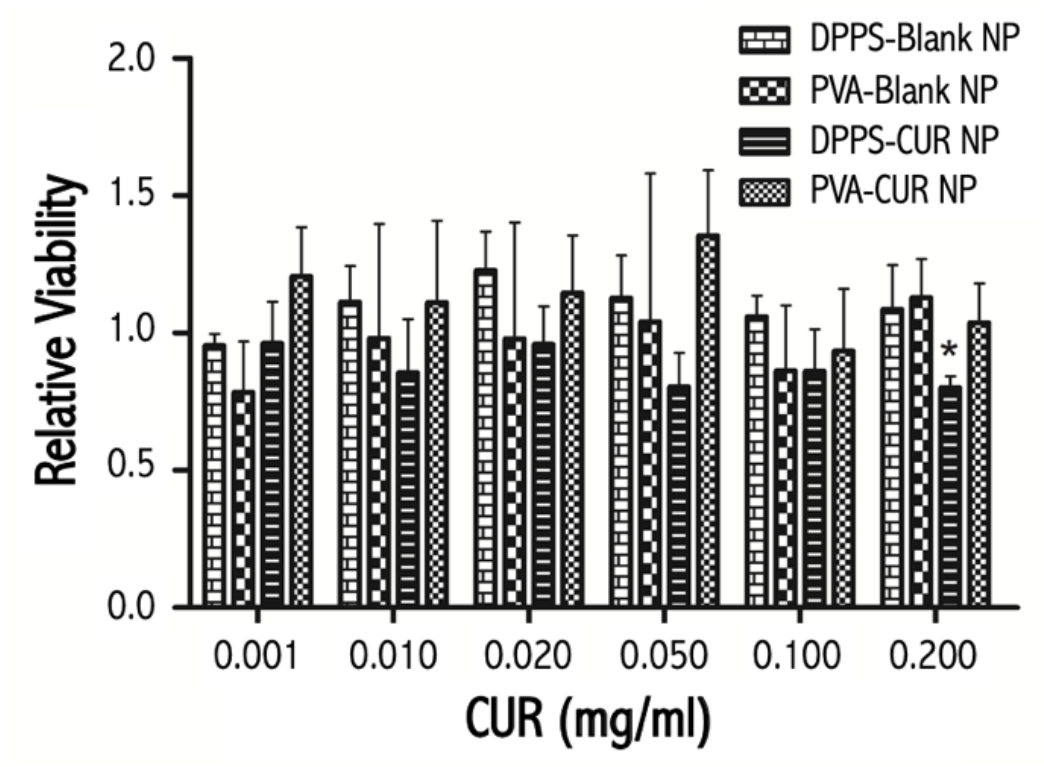

Figure 3.5. Cytotoxicity analysis (relative viability) of nanoparticle systems on RAW 264.7 macrophages after 48 hours of exposure compared to control $\left({ }^{*} \mathrm{p}<0.05\right)$.

\subsubsection{Nitrite Analysis}

Following 48-hour incubation of NP samples on cells, CUR-loaded and blank (control) NP groups produced significantly lower NO in comparison to LPS ( $\mathrm{p}<$ 0.0001) (Figure 3.6). The NP groups were the same as the control group (media only) statistically, indicating little to no production of NO (208). 


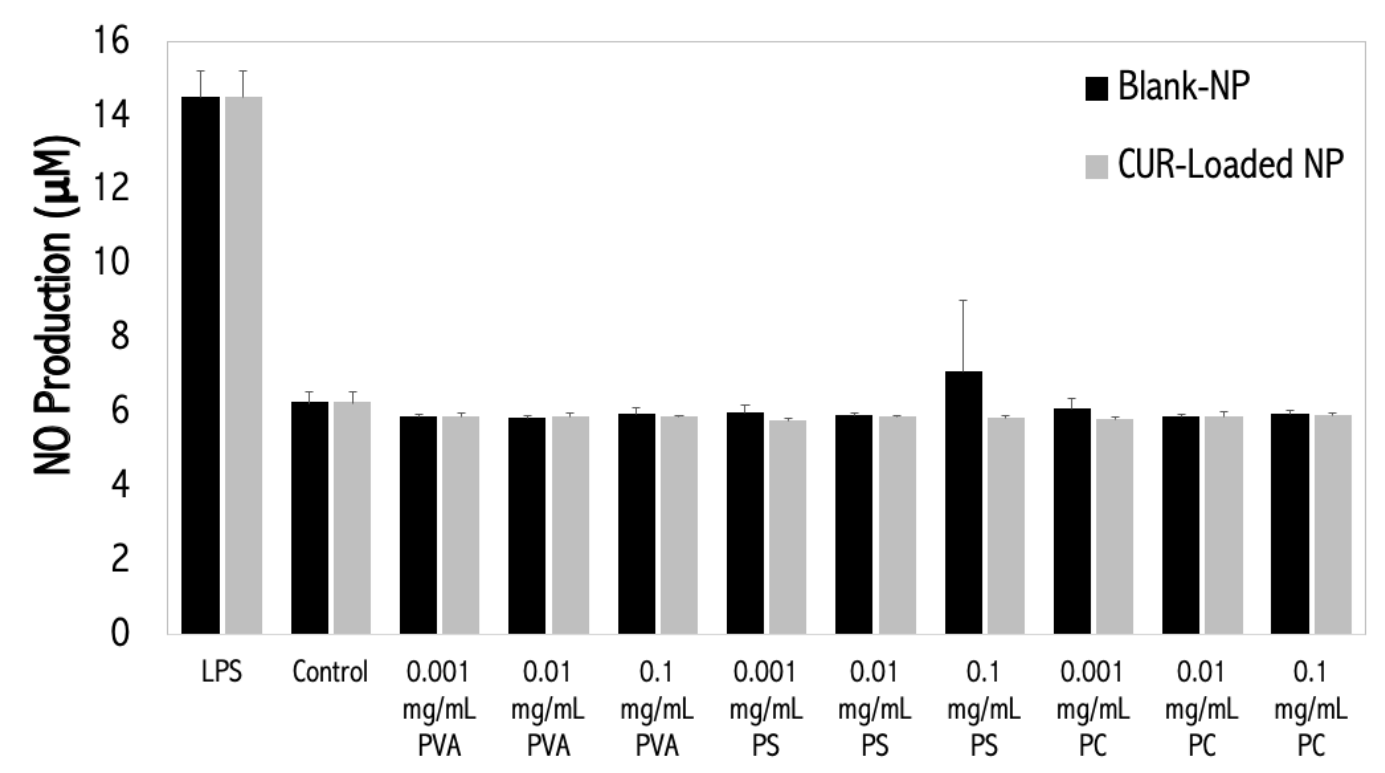

Figure 3.6. Nitric oxide (NO) production from RAW 264.7 macrophages incubated for 48 hours with varying concentrations of nanoparticle formulations or LPS. No statistical significance was observed between the groups and control group (no treatment) and all were statistically lower than the LPS sample ( $<<0.0001)$.

\subsubsection{In Vitro Cellular Uptake}

The cellular uptake of CUR-loaded NP was evaluated in RAW 264.7 macrophages and A549 adenocarcinoma cells for up to 3 hours as shown in Figure 3.7. Exploiting the fluorescent properties of CUR, the presence of CUR-loaded NP within cells can easily be detected via fluorescence imaging and spectroscopy. After 1 hour of exposure, significantly more DPPS-coated NP were uptaken by macrophages and A549 cells in comparison to PVA-coated NP, DPPC-coated NP, and raw CUR (p $<0.0001)$. For both cell types and all formulations, uptake was not statistically different between 1 and 3-hour exposures. In addition, significantly more NP were 
uptaken in RAW macrophages in comparison to A549 cells for all NP formulations. Confocal microscopy confirmed fluorescence spectroscopy quantification, showing higher fluorescence in macrophages in comparison to A549 cells, indicating increased NP uptake. In addition, more NP were uptaken in DPPS-coated NP in comparison to DPPC- and PVA-coated systems in RAW and A549 cells. Images indicate that CURloaded NP were primarily uptaken into the cytoplasm of macrophages, especially for DPPS-CUR NP. On the other hand, there were minimal NP located in the cytoplasm of A549 cells; instead the NP were more readily located in the cellular membranes.
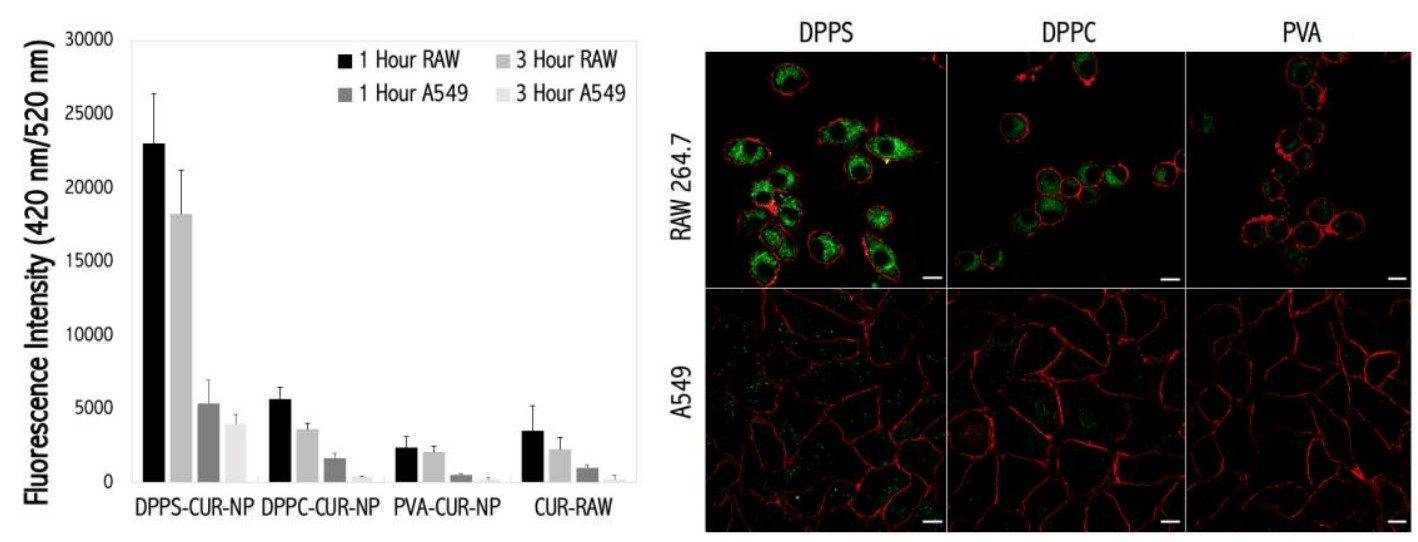

Figure 3.7. (Left) Fluorescence spectroscopy analysis of nanoparticle uptake into cells and (Right) representative confocal images of nanoparticle uptake by RAW 264.7 macrophages and A549 cells (scale bar $=10 \mu \mathrm{m})$. 


\subsection{Discussion}

\subsubsection{Nanoparticle Design and Analysis}

The use of Ac-Dex as a biodegradable NP core for drug delivery can allow for a system with tunable and/or triggered degradation (19). For the described NP systems, the acetal coverage of Ac-Dex was similar to previous studies $(45,53,54)$, whereas the CAC ratio was slightly lower. The resulting Ac-Dex was favorable for the given application as the adequate acetal coverage imparts hydrophobicity to Ac-Dex, allowing for $\mathrm{NP}$ formulation and the lower $\mathrm{CAC}$ ratio will allow for faster drug release at acidic $\mathrm{pH}(45,49,53,54)$.

The increase in diameter and PDI from PVA-coated NP to DPPS-coated NP is likely due to the presence of the DPPS coating around the Ac-Dex core. It has been shown that an increase in NP diameter can lead to an increase in the uptake of NP into macrophages, where particles $200 \mathrm{~nm}$ in diameter or greater are often subjected to a greater amount of macrophage uptake $(57,84,209)$. For this study, larger NP were desired and successfully formed (up to $350 \mathrm{~nm}$ for DPPS-coated NP), which was advantageous since increased macrophage uptake was an objective (43). While DPPScoated NP exhibited higher PDI values then PVA-coated NP, these values were still low, indicating that all of the formulated NP systems were homogenous with respect to size.

The surface charge of the NP, as indicated by zeta potential, decreased significantly (from -3 to $-40 \mathrm{mV}$ ) upon coating with DPPS. The negative surface charge on the DPPS-loaded NP is indicative of the polar head of DPPS being exposed to the outer environment, similar to how apoptotic cells present DPPS to the outer 
leaflet of cells prior to engulfment by macrophages $(182,197)$. The highly negatively surface charge is significantly lower than the $-25 \mathrm{mV}$ threshold of being functionally negative, indicating that the NP are less likely to aggregate due to electrostatic repulsion $(79,80)$. It should be noted that DPPC-coated NP were also lower than the $25 \mathrm{mV}$ threshold, indicating a functionally negative charge. In addition, there were no differences in size, PDI value, or zeta potential upon the loading of CUR on the NP core, indicating that the loading of the model drug had no significant effect on the particle systems with respect to these characteristics.

CUR was encapsulated in the NP for two purposes: 1) to provide a model small molecule that is easy to encapsulate owing to its hydrophobicity and 2) the inherent fluorescence of CUR within the NP allows for easy fluorescence imaging and detection. The encapsulation efficiency (EE) of CUR in the NP was lower than previously reported values, which have ranged from 50-88\% (183, 185, 203). However, since CUR was used as a model small molecule, the EE values were satisfactory for this study. Upon coating the NP with DPPS, CUR loading decreased slightly but the encapsulation efficiency stayed the same, indicating that the DPPS coating did not significantly affect CUR loading.

The SEM and TEM micrographs indicate that the particles are spherical in shape and monodisperse, which correlates to the PDI values for the systems. The DPPS-CUR NP seem to have slightly more coating on the NP surfaces, which could be due to the increased presence of DPPS. 


\subsubsection{Coating Quantification}

DPPS was selected to coat the nanoparticles owing to its ability to stimulate an "eat-me" signal on apoptotic cells $(10,82,174,175,180)$. For cell studies, DPPC-NP were produced as a control lipid system. Initially, the NP systems were coated with PVA because it is one of the most widely used polymers in the pharmaceutical industry (206) and has been extensively used as a NP coating agent due to the steric effects PVA coating imparts on NP, inhibiting aggregation. During the synthesis of phospholipid-coated NP, a significant portion of the PVA was washed away prior to phospholipid coating, since there is significantly less PVA present on phospholipidcoated NP in comparison to PVA NP (Figure 3.2). It is also likely that the phospholipid coating shielded the remaining PVA upon adsorption to the NP surface, as indicated by the low mass presence of PVA on phospholipid-coated NP the high mass presence of total phospholipid on the NP, and the changes in surface charge

\section{(Figure 3.2).}

\subsubsection{Thermal Analysis of Nanoparticle Formulations}

Dry-state DSC thermograms indicated the presence of DPPS in the DPPScoated NP via the bilayer phase transition peak that occurred at $90^{\circ} \mathrm{C}$ for the NP, which is slightly lower than the raw DPPS endothermic peak present at $99^{\circ} \mathrm{C}$. This transition signifies the presence of bilayer formation of the phospholipid around the Ac-Dex polymer core in its dry state. The decrease in the transition temperature is likely due to the interaction of the multiple components in the formulation. As the transition temperatures of the NP formulations were just below or well above $100^{\circ} \mathrm{C}$, 
they are likely to be stable in the dry state and can be easily reconstituted before use (145). The endothermic peak that raw CUR exhibited at $177^{\circ} \mathrm{C}$ was not observed in CUR-loaded NP. According to our previous research (data not shown), this is likely due a limitation in DSC detection with relation to the actual amount of CUR in the samples.

In studies using liposomal formulations, DSC is used to assess the transition temperature(s) in the systems in order to determine the presence and state of bilayers and the differences associated between blank liposomes and modified/drug-loaded systems $(204,205)$. For wet-state analysis (Figure 3.3C), there was no endothermic peak present around $50-60^{\circ} \mathrm{C}$, which is the temperature at which the gel/crystalline phase transition occurs for PS bilayers in aqueous solutions $(193,210)$. Such a phenomenon could have occurred based on the preparation method used, as described previously. In certain cases, self-assembly of polymer-lipid structures can occur via hydrophobic interaction between the lipid tails and a polymeric core simultaneously with hydrophilic interactions between the polar head and external environment (211). Due to the concentration dependency of DSC, it is more likely that the DPPS concentration may have been too low to be detectable by thermal analysis, like that of the CUR detection limit mentioned previously. It should be noted that the curvature of the thermograms in Figure 3.3C corresponds to the evaporation of water, which is not shown in the thermogram. Overall, the results indicate the presence of DPPS on the surface of the NP, further strengthening what has been previously described with respect to successful DPPS coating of the described systems. 


\subsubsection{Curcumin Release from Nanoparticles}

The release from CUR from PVA- and DPPS-coated NP was evaluated to demonstrate the ability of the system to provide sustained release of a therapeutic agent and to show the acid-sensitive nature of Ac-Dex. Ac-Dex is known to degrade more quickly in acidic environments (19). In a previous study, Ac-Dex particles released $60 \%$ of their camptothecin payload after 7 days (49) and $15 \%$ of rapamycin after 10 days (43) at $\mathrm{pH}$ of 7.4. Comparatively, at $\mathrm{pH}$ values of 5 or lower, Ac-Dex particles released $100 \%$ of their content in as little as 24 hours $(36,43,45)$. For this study, at $\mathrm{pH} 5.35,9.3 \%$ of CUR was initially released from DPPS-CUR NP and $29.2 \%$ was released after 1 hour, followed by a sustained release of cargo of $67.8 \%$ after 24 hours, when equilibrium is reached. At pH 7.4, both NP systems exhibited minimal CUR release, demonstrating the stability of Ac-Dex in neutral pH. However,

both systems exhibited triggered release at $\mathrm{pH} 5.35$, exhibiting a significantly higher total release in comparison to their $\mathrm{pH} 7.4$ counterparts $(\mathrm{p}<0.001)$. Overall, these data indicate a more stable NP formulation at $\mathrm{pH} 7.4$ and the potential for triggered release at $\mathrm{pH} 5.35$, corresponding to stability in circulation and burst release in macrophages, respectively.

\subsubsection{Cytotoxic Analysis and Macrophage Uptake of Nanoparticles}

To assess the in vitro cytotoxicity of the NP formulations on RAW 264.7 macrophages, the cells were exposed to the systems for 48 hours. It was observed that the NP systems were not toxic to the cells, however, a slight, but significant, decrease in viability of RAW 264.7 cells for DPPS-CUR NP at $0.2 \mathrm{mg} / \mathrm{ml}$ was observed 
(Figure 3.5). This decrease in viability was minimal and could easily be overcome by using lower concentrations of the particles in future studies. Overall, these results indicate the safety of the described NP for a time period longer than necessary for NP uptake into macrophages, indicating that they are safe to use in the intended fashion.

Nanomaterials may provoke immune and inflammation responses following interactions with multiple biological entities (57). To assess inflammatory and immune response stimulation, NO production was measured using a Griess assay, where LPS was used as a positive control to stimulate NO production in RAW macrophages. It was observed that NP at varying concentrations stimulated significantly less NO production in comparison to LPS and resulted in NO concentrations comparable to that of the control (media only). Results indicated that blank NP caused NO production at concentrations similar to that of the CUR-loaded NP. These data indicate both blank and CUR-loaded formulations produced minimal NO and that CUR did not play an active role in the inhibition of NO production.

Since the purpose of this study was to formulate acid-sensitive NP capable of enhanced macrophage uptake, the ability of DPPS-coated NP to be uptaken by macrophages was evaluated, with A549 cells acting as control tissue. PVA and DPPCcoated NP were used as other coatings for comparison to DPPS. Several environmental and physicochemical factors are known to influence the cellular uptake of NP into macrophages. The preferential uptake of DPPS-CUR NP by macrophages in comparison to PVA-CUR NP and DPPC-CUR NP is likely due to the DPPS coating on the Ac-Dex polymeric core. Confocal microscopy in tandem with fluorescence quantification of CUR-loaded NP within the cells confirm the uptake of DPPS-CUR- 
NP and localization to the cytoplasm of macrophages. The presence of DPPS on the cellular surface is known to cause an "eat-me" signal in cells that are transitioning toward an apoptotic state, causing macrophages to identify and phagocytose the dying cells $(82,173-175,183)$. Therefore, in terms of recognition, the DPPS coating on the Ac-Dex NP was identified by the macrophages, which produced the necessary phagocytotic action, resulting in cellular uptake of the particles $(10,82,182,183,194$, 196). In most cases, apoptosis is initiated by the presence DPPS and other signaling pathways such as receptor-ligand interactions or identification of PS binding proteins produced by phagocytes. However, there is a debate as to whether DPPS recognition by itself is sufficient for the uptake of apoptotic cells by phagocytotic cells $(174,194)$. In the case of nanoparticles, is has been shown that a DPPS coating alone is sufficient for uptake, as seen in this current study and otherwise $(10,180,183)$.

Another consideration for cellular uptake of the described particles is in terms of their physicochemical characteristics. Stimulation of phagocytosis of nanoparticlebased delivery systems via macrophages is also dependent on the size and shape of the particles. Since the DPPS-CUR NP were greater than $300 \mathrm{~nm}$ in diameter, it is likely that these NP were phagocytosed not only because of the DPPS layer, but also because of their size $(43,84,212)$. It is well known that surface charge can play a role in biological interactions. Interestingly, surface charge did not play a role in the uptake of the NP, as shown by the similar charges of DPPC and DPPS-coated NP that produced significantly different uptake quantities $(79,80,84)$. This phenomenon could also explain the minimal phagocytosis of the PVA-coated NP and confirm the effect of DPPS on the uptake of the NP. Overall, the results indicate the successful uptake of 
DPPS-coated NP into macrophages, indicating that these systems could be used for the treatment of a multitude of diseases involving infected cells.

\subsection{Conclusions}

Macrophages can harbor infectious agents that lead to potentially fatal diseases such as TB and HIV. Due to this, treatment of these diseases is challenging and therefore, the goal of this study was to design a delivery system that would enhance the uptake of nanoparticles into macrophages, allowing for targeted delivery, a decrease of systemic side effects, and decreased treatment times. Results showed that both PVA-coated and DPPS-coated NP were monodisperse and that there was a significant increase in size upon DPPS coating. The surface charge of the DPPS NP was $-40 \mathrm{mV}$, indicating that DPPS was present on the surface of the NP. This was further confirmed via PVA quantification, which indicated minimal amounts of PVA on the DPPS NP. DSC results confirmed the presence of a DPPS bilayer on the NP surface and the likely stability of the NP during synthesis and storage conditions. CUR-loaded NP successfully encapsulated CUR and this agent was more quickly released in acidic conditions in comparison to neutral $\mathrm{pH}$ owing to the acid sensitivity of Ac-Dex. In addition, CUR-loaded NP exhibited sustained release of CUR. PVAcoated NP were not readily phagocytosed by macrophages, whereas DPPS-coated NP were phagocytosed within 1 hour of exposure, showing the physiological relevance of the DPPS coating for the enhanced delivery to macrophages. These data show that the particles can potentially deliver therapeutic agents throughout the body with fewer concerns of systemic drug exposure, and when exposed to macrophages, the particles 
are capable of being phagocytosed relatively quickly. Therefore, the combination of Ac-Dex and DPPS can be a viable option for targeted delivery for macrophageassociated diseases. 


\section{CHAPTER 4}

\section{SUSTAINED RELEASE OF WATER-SOLUBLE THERAPEUTICS VIA DRY POWDER AEROSOL ACETALATED DEXTRAN MICROPARTICLES}

Submitted for publication in Drug Development and Industrial Pharmacy in April 2019.

Nishan K. Shah ${ }^{1}$, Sweta K. Gupta ${ }^{2}$, Zimeng Wang ${ }^{2}$, Andrew Le Campion², Samantha
A. Meenach ${ }^{1,2, *}$

${ }^{1}$ University of Rhode Island, College of Pharmacy, Department of Biomedical and Pharmaceutical Sciences, Kingston, RI 02881

${ }^{2}$ University of Rhode Island, College of Engineering, Department of Chemical Engineering, Kingston, RI 02881 


\subsection{Abstract}

Objective: The objective of this study was to design and characterize aerosol microparticles capable of providing sustained release of the model water-soluble compound sulforhodamine B (SRB) in addition to achieving aerosol dispersion.

Significance: Modulating the release of water-soluble compounds remains an ongoing challenge in pulmonary drug delivery

Methods: SRB was mixed with water to form an aqueous solution, while acetalated dextran (Ac-Dex) was mixed with isopropyl alcohol to form an organic solution. These two solutions were mixed together, resulting in the precipitation of SRB, and the solution was spray dried to produce dry powder microparticles (MP). MP were characterized for their morphology, size, release kinetics at different $\mathrm{pH}$, aerosol dispersion, and cellular interactions.

Results: Ac-Dex MP exhibited corrugated morphology with diameters around $1 \mu \mathrm{m}$ and experimental mass median aerodynamic diameters from 2.06 to $2.86 \mu \mathrm{m}$. With respect to aerosol dispersion, the MP deposited in all stages of a Next Generation Impactor, with $>90 \%$ fine particle fraction. MP exhibited encapsulation efficiencies > $129 \%$ with SRB loading values up to $16.7 \mu \mathrm{g}$ SRB/mg MP. Release kinetics demonstrated sustained release of $\mathrm{SRB}$ at $\mathrm{pH} 7$ and faster release at $\mathrm{pH}$ 5. In vitro experiments showed minimal cytotoxicity, successful uptake of MP in epithelial cells, and no disruption to the integrity of epithelial monolayers up MP exposure.

Conclusions: Overall, the Ac-Dex MP systems demonstrated the ability to provide sustained the release of a water-soluble therapeutics in addition to aerosol delivery of the particles to the lungs. 
4.2 Keywords: Aerosol; sustained released; pulmonary drug delivery; microparticles; water-soluble compounds; Acetalated dextran

4.3 Abbreviations: 2-methoxypropene (2-MOP), acetalated dextran (Ac-Dex), cyclicto-acyclic ration (CAC), dichloromethane (DCM), dimethyl sulfoxide (DMSO), deionized (DI) water, differential scanning calorimetry (DSC), dynamic light scattering (DLS), encapsulation efficiency (EE), Karl Fischer Titration (KFT), Microparticles (MP), Net Generation Impactor (NGI), phosphate buffered saline (PBS), poly(vinyl alcohol) (PVA), poly(lactic-co-glycolic acid) (PLGA), ptoluenesulfonate (PPTS), polarized light microscopy (PLM), scanning electron microscopy (SEM), sulforhodamine B (SRB), triethylamine (TEA)

\subsection{Introduction}

Lung diseases remain a persistent health concern and as a result, it is necessary to continue to develop new drug delivery systems to improve the treatments in this area $(145,213)$. Dry powder aerosol formulations are an important option in the treatment of pulmonary diseases since they promote localized delivery, avoidance of first-pass metabolism, concentrated drug dosing, and a rapid onset of action, thereby reducing systemic toxicity and dosing frequency $(135,214)$.

Two challenges in formulating dry powder therapeutics are the diversity in delivered compounds, especially with respect to their solubility and/or crystallinity (145), and the need for formulations capable of sustained release (130). Highly watersoluble agents including antibiotics (153), anti-cancer small molecules $(215,216)$, proteins $(48,217,218)$, and gene-related therapeutics $(209,213,219)$ have been 
formulated as dry powders. However, owing to their solubility and the nature of particle formulation methods, water-soluble therapeutics can be challenging to formulate. In addition, their high solubility in aqueous solutions results in fast dissolution (220) and this can result in unfavorable pharmacokinetic and pharmacodynamic outcomes $(2,130,220,221)$. Also, water soluble therapeutics are usually administered orally or parenterally routinely to maintain favorable pharmacokinetics. Frequent systemic administration of these therapeutics can result in toxicity (130). Therefore, it is desirable to develop formulations capable of localizing and controlling the release of water-soluble therapeutics to decrease systemic administration (and side effects) as well as increasing the control over the release of encapsulated cargo., especially for pulmonary drug delivery applications.

To modulate the release of therapeutics, it is common practice to use polymer carriers such as poly(lactic-co-glycolic acid) (PLGA) (2). Unfortunately, PLGA-based formulations often exhibit significant burst release of its cargo and the degradation of PLGA can result in undesirable changes in the microenvironment. An alternative option is acetalated dextran (Ac-Dex), a synthetic derivative of the FDA-approved excipient dextran (19). Using a one-step reaction, hydroxyl groups on dextran are acetalated, thereby modifying the polymer to be hydrophobic $(19,40,222)$. The reaction time of Ac-Dex can be easily controlled, resulting in tunability of the ratio of cyclic-to-acyclic acetal groups $(35,40,50,222)$, which can be used to control the degradation rates of Ac-Dex drug delivery systems. The degradation of Ac-Dex occurs within a few hours in an acidic environments and as long as weeks in a neutral environment, making it ideal for sustained release of therapeutics, depending on the 
application (45). In addition, the degradation by-products of Ac-Dex have been shown to be non-toxic and does not produce immunogenic and inflammatory responses (19), which is especially important for pulmonary applications.

Ac-Dex has been used in a wide variety of drug delivery applications, including the delivery of anticancer agents $(49,50)$, antibiotic and antiviral agents $(41$, 47, 51), and immunosuppressive small molecules (43). In addition, our previous studies have demonstrated the successful design of several Ac-Dex formulations for sustained release of therapeutics in the lungs $(53,54,223)$. Despite this work, there is no comprehensive study on the design and characterization of Ac-Dex-based systems that can allow for sustained release of water-soluble therapeutics within the lungs. The goal of the present study is the development and characterization of novel Ac-Dex microparticle (MP) systems. We hypothesize that the resulting Ac-Dex MP can allow for successful encapsulation and sustained of a model water-soluble compound, while allowing for effective aerosol dispersion of the particles for pulmonary applications.

The novelty of the formulations lies in the use of Ac-Dex for formulate aerosol MP to encapsulate a water-soluble compound, the formulation method itself (aqueous in organic precipitation), and the successful encapsulation and release of a watersoluble compound, sulforhodamine B (SRB). SRB was chosen as a model compound owing to its high solubility in water (> $100 \mathrm{mg} / \mathrm{ml}$ ) and fluorescent properties. The ensuing formulation could be utilized for a wide variety of water-soluble compounds, demonstrating the significance of the described study and results.

\subsection{Materials and Methods}

\subsubsection{Materials}


Detailed information on the materials can be found in Supplemental Information.

\subsubsection{Synthesis and characterization of acetalated dextran}

Ac-Dex was synthesized using previously described methods with minor modifications (45). $1 \mathrm{~g}$ of lyophilized dextran and 25-30 $\mathrm{mg}$ of PPTS were dissolved in $10 \mathrm{~mL}$ of DMSO under nitrogen gas. The reaction was carried out for 5 minutes or 3 hours by adding $5 \mathrm{~mL}$ or $6.5 \mathrm{~mL}$ of 2 -MOP, respectively. The reaction was quenched with $1 \mathrm{~mL}$ of TEA. The polymer was precipitated in basic water (pH 9), vacuum filtered, frozen overnight, and lyophilized. Corresponding to their degradation rates, products of 5-minute and 3-hour reaction times are designated as Fast-Ac-Dex and Slow-Ac-Dex, respectively. The cyclic-to-acyclic (CAC) ratio of Ac-Dex and acetal coverage were verified using ${ }^{1} \mathrm{H}$ NMR (Bruker $300 \mathrm{MHz}, \mathrm{NMR}, \mathrm{MA}$ ) as previously described (45).

\subsubsection{Microparticle synthesis via spray drying}

MP systems were prepared via spray drying using a Büchi B-290 spray dryer (Büchi Labortechnik, AG, Switzerland) in closed mode (organic solution; Ac-Dex MP systems) or open mode (aqueous solution; mannitol system) as seen in Figure 4.1. It should be noted that pure SRB was not spray dried in this study. Instead, SRB encapsulated in mannitol was used as the control due to their combined water solubility. For Ac-Dex MP, $1 \mathrm{~mL}$ of $4 \mathrm{mg} / \mathrm{mL}$ SRB solution in deionized (DI) water was slowly injected into a spinning solution of $400 \mathrm{mg}$ Ac-Dex in $20 \mathrm{~mL}$ of isopropanol to create a water-organic (W/O) solution prior to spray drying, resulting in 
an initial loading of $1 \% \mathrm{w} / \mathrm{w}$ SRB. For mannitol MP, $1 \mathrm{ml}$ of $4 \mathrm{mg} / \mathrm{mL}$ SRB was added to a $20 \mathrm{mg} / \mathrm{mL}$ solution containing approximately $400 \mathrm{mg}$ of mannitol $(1 \% \mathrm{w} / \mathrm{w}$ SRB). SRB-excipient solutions were spray dried using optimized conditions (Table 4.1) based on a previous study (44). While the type of excipient, set inlet temperature, and pump rate were varied, the following conditions were kept constant: $0.7 \mathrm{~mm}$ nozzle diameter, aspiration rate of $40 \mathrm{~m}^{3} / \mathrm{h}$, nozzle cleaner rate of 0 , and gas flow rate of $414 \mathrm{~L} / \mathrm{h}$ using ultra high pure nitrogen.

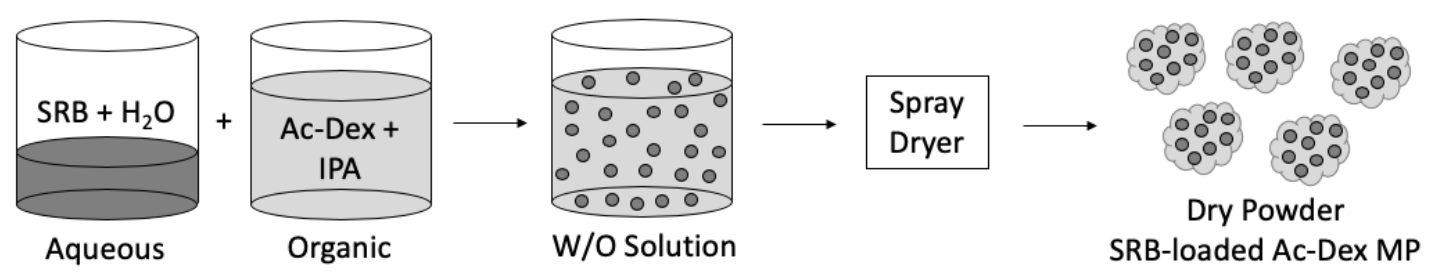

Figure 4.1. Schematic of microparticle preparation including mixing of aqueous solution containing sulforhodamine (SRB) and water and organic solution containing acetalated dextran (Ac-Dex) and isopropyl alcohol (IPA) to form a water/organic (W/O) solution prior to spray drying and microparticle formation.

Table 4.1. Spray drying parameters for particle sets, including the reaction time used for acetalated dextran (Ac-Dex) synthesis, spray drying parameters (set inlet temperature and pump rate), and actual inlet and outlet temperatures during the spray drying process. The particle naming convention is as follows: 'Fast' refers to 5-minute Ac-Dex (fast degrading), 'Slow' refers to 3-hour Ac-Dex (slow degrading), the temperature value is the set inlet temperature setting, and 'LowP', 'MedP', and 
'HighP' refers to 10,30 , and $100 \%$ pump rates, respectively (mean \pm standard deviation, $\mathrm{n}=3$ ).

\begin{tabular}{|c|c|c|c|c|c|}
\hline System & $\begin{array}{c}\text { Ac-Dex } \\
\text { Reaction } \\
\text { Time }\end{array}$ & $\begin{array}{c}\text { Set Inlet } \\
\text { Temperature } \\
\left({ }^{\circ} \mathrm{C}\right)\end{array}$ & $\begin{array}{c}\text { Pump } \\
\text { Rate } \\
(\%)\end{array}$ & $\begin{array}{c}\text { Actual Inlet } \\
\text { Temperature } \\
\left({ }^{\circ} \mathrm{C}\right)\end{array}$ & $\begin{array}{c}\text { Actual Outlet } \\
\text { Temperature } \\
\left({ }^{\circ} \mathbf{C}\right)\end{array}$ \\
\hline $\begin{array}{c}\text { Fast- } 100^{\circ} \mathrm{C}- \\
\text { MedP }\end{array}$ & 5-minute & 100 & 30 & $100.8 \pm 1.9$ & $57.8 \pm 2.2$ \\
\hline $\begin{array}{c}\text { Fast- } 150^{\circ} \mathrm{C}- \\
\text { MedP }\end{array}$ & 5-minute & 150 & 30 & $150.1 \pm 0.8$ & $77.8 \pm 6.7$ \\
\hline $\begin{array}{c}\text { Fast- } 100^{\circ} \mathrm{C}- \\
\text { HighP } \\
\end{array}$ & 5-minute & 100 & 100 & $102.6 \pm 2.9$ & $57.1 \pm 6.6$ \\
\hline $\begin{array}{c}\text { Slow- } 100^{\circ} \mathrm{C}- \\
\text { MedP }\end{array}$ & 3-hour & 100 & 30 & $103.6 \pm 2.5$ & $56.2 \pm 2.2$ \\
\hline $\begin{array}{c}\text { Slow- } 150^{\circ} \mathrm{C}- \\
\text { MedP }\end{array}$ & 3-hour & 150 & 30 & $153.8 \pm 2.3$ & $83.1 \pm 3.6$ \\
\hline $\begin{array}{c}\text { Slow- } 100^{\circ} \mathrm{C}- \\
\text { HighP } \\
\end{array}$ & 3-hour & 100 & 100 & $104.2 \pm 2.0$ & $59.2 \pm 3.5$ \\
\hline $\begin{array}{c}\text { Man- } 100^{\circ} \mathrm{C}- \\
\text { LowP }\end{array}$ & --- & 100 & 10 & $101.4 \pm 1.7$ & $55.2 \pm 1.6$ \\
\hline
\end{tabular}

\subsubsection{Morphology, size, and tapped density analysis}

Images of MP systems were obtained using a Zeiss Sigma VP Field EmissionScanning Electron Microscope (FE-SEM, Germany) following a previously described preparation method, and were taken at $10,000 \mathrm{x}$ and $5 \mathrm{kV}$ (54). To determine the geometric diameter (dg) of the MP, particles in SEM micrographs were analyzed using ImageJ software (Systat, San Jose, CA, USA) using at least 100 MP of each system. The hydrodynamic diameter of MP systems was analyzed using a Malvern Nano Zetasizer (Malvern Instruments, Worcestershire, UK). Ac-Dex MP were diluted to $0.25 \mathrm{mg} / \mathrm{mL}$ in DI water and were analyzed at $25^{\circ} \mathrm{C}$ and $90^{\circ}$. Mannitol MP samples were analyzed in the same manner using chloroform. The tapped density of particles was measured using a previously described method (45). The density of the MP systems was calculated via the following equation: 


$$
=\frac{m}{V}
$$

where $\rho$ is the tapped density, $\mathrm{m}$ is the mass, and V is the volume occupied by MP. The theoretical mass median aerodynamic diameter (MMADT) of the MP systems were then calculated via:

$$
M M A D_{T}=d_{g} \sqrt{r e f}
$$

where $\mathrm{dg}$ is the geometric diameter, $\rho$ is the tapped density, and $\rho_{\text {ref }}$ is the reference density of solid polymer $\left(1 \mathrm{~g} / \mathrm{cm}^{3}\right)$.

\subsubsection{In vitro aerosol performance}

In vitro aerosol performance of MP systems were evaluated using a Next Generation Impactor (NGI, MSP Corporation, Shoreview, MN) as previously described (54). For $\mathrm{Q}=60 \mathrm{~L} / \mathrm{min}$, cutoffs for each stage of the NGI are $8.06 \mu \mathrm{m}$ (stage 1), $4.46 \mu \mathrm{m}$ (stage 2), $2.82 \mu \mathrm{m}$ (stage 3), $1.66 \mu \mathrm{m}$ (stage 4), $0.94 \mu \mathrm{m}$ (stage 5), $0.55 \mu \mathrm{m}$ (stage 6) and $0.34 \mu \mathrm{m}$ (stage 7). Fine particle fraction (FPF), respirable fraction $(\mathrm{RF})$, and emitted dose (ED) were calculated using the following equations:

$$
\text { Fine Particle Fraction }(\mathrm{FPF})=\frac{\text { Mass of MP on stages } 2 \text { through } 7}{\text { Total MP mass on all stages }} \times 100 \%
$$




$$
\text { Respirable Fraction }(\mathrm{RF})=\frac{\text { Mass of MP on stages } 2 \text { through } 7}{\text { Initial MP mass loaded into capsules }} \times 100 \%
$$

$$
\text { Emitted Dose }(E D)=\frac{\text { Initial MP mass in capsules }- \text { Final MP mass in capsules }}{\text { Initial MP mass in capsules }} \times 100 \%
$$

The experimental mass median aerodynamic diameter $\left(\mathrm{MMAD}_{\mathrm{E}}\right)$ and geometric standard deviation (GSD) for the MP systems were determined using a Mathematica ${ }^{\circledR}$ program written by Dr. Warren Finlay (224).

\subsubsection{Crystallinity and phase transition analysis}

Thermal phase transitions of MP systems and raw materials were analyzed via differential scanning calorimetry (DSC) using a TA Q10 DSC system (TA Instruments, New Castle, DE) connected to an RSC-90 cooling accessory. 3-5 mg of sample were hermetically sealed in aluminum pans and were placed into the DSC furnace. Samples were heated from $0-200^{\circ} \mathrm{C}$ at a rate of $10^{\circ} / \mathrm{min}(45)$. The crystallinity of MP systems and raw materials were examined by X-ray diffraction (XRD) using a Rigaku Multiflex X-ray diffractometer (The Woodlands, TX) as described previously with a scan range of $5-60^{\circ}$ in $2 \Theta$ with a width of 0.1 and scan rate of $1 \%$ min. (45). Images of MP systems and raw materials were observed for birefringence via polarized light microscopy (PLM) using an AmScope polarized microscope (Irvine, CA) (225). Samples were mounted onto microscope slides, smeared with mineral oil for dispersion, and imaged at 10x magnification at room temperature. 


\subsubsection{Analysis of water content via Karl Fischer titration}

Water content of the MP systems was quantified via Karl Fischer (KF) titration using a $737 \mathrm{KF}$ coulometer (Metrohm, Riverview, FL, USA). 3 mg of powder was dissolved in methanol and the resulting solution was injected into the KF cell filled with Hydranal ${ }^{\circledR}$ and the amount of water was analyzed. Pure solvent was also injected as a background sample.

\subsubsection{Encapsulation efficiency and drug loading analysis}

To determine the amount of SRB encapsulated in the MP systems, MP were dissolved in methanol or deionized water $(1 \mathrm{mg} / \mathrm{mL})$ and quantified via fluorescence spectroscopy using a Cytation 3 plate reader (BioTek, Winooski, VT, USA) at 565/586 nm (ex/em) (226). The encapsulation efficiency (EE) and drug loading (DL) of MP were calculated using the following equations:

$$
\begin{gathered}
\mathrm{EE}=\frac{\text { Actual mass of SRB in MP }}{\text { Theoretical mass of SRB in MP }} \times 100 \% \\
\mathrm{DL}=\frac{\text { Mass of SRBin MP }}{\text { Mass in MP }}
\end{gathered}
$$

\subsubsection{Drug release and release kinetic analysis}

Release of SRB from MP was carried out using a centrifugation technique. 10 $\mathrm{mg} / \mathrm{mL}$ samples were created by dispersing MP in phosphate buffered solution (PBS, 
$\mathrm{pH}$ 7.4) or sodium acetate buffer $\left(\mathrm{pH}\right.$ 5.35). The suspensions were incubated at $37^{\circ} \mathrm{C}$ and $100 \mathrm{rpm}$ and centrifuged at 23,102 x g for 5 minutes at predetermined time points. $400 \mu \mathrm{L}$ of the supernatant was collected and frozen at $-20^{\circ} \mathrm{C}$ and $400 \mu \mathrm{L}$ of fresh buffer was added to the particle solution prior to re-dispersion. Supernatant samples were supplemented with equal parts methanol or water to solubilize any remaining excipient. Analysis was carried out via fluorescence spectroscopy and normalized SRB release was calculated as follows:

$$
\text { Normalized SRB Release }=\frac{\text { Mass Released at } \mathrm{t}=\text { time }}{\text { Total Mass Released }} \times 100 \%
$$

Drug release kinetics of SRB released from the MP were evaluated using six different models, including: zero-order, first-order, Higuchi, Korsmeyer-Peppas (a.k.a. the power law), Peppas-Sahlin, and Corrigan. The model parameters and coefficient of determination $\left(\mathrm{R}^{2}\right)$ for each model were determined using least squares regression. For zero-order, first-order, Higuchi, and Korsmeyer-Peppas models, the equations were linearized, and the slope, intercept, and $\mathrm{R}^{2}$ values were determined using the 'linest' function in Microsoft Excel prior to calculation of the model parameters. For PeppasSahlin and Corrigan, the model parameters and $\mathrm{R}^{2}$ values were determined using the 'fminsearch' function in MATLAB. A detailed description of the release models and parameters can be found in Supplemental Information.

\subsubsection{Cell Culture}


A549, H441, and Calu-3 cells were obtained from ATCC (Manassas, Va, USA). A549 cells were maintained at $37^{\circ} \mathrm{C}$ and $5 \% \mathrm{CO}_{2}$ in Dulbecco's modified Eagle's medium (DMEM) supplemented with 10\% (v/v) fetal bovine serum, $100 \mathrm{U} / \mathrm{ml}$ penicillin, $100 \mu \mathrm{g} / \mathrm{ml}$ streptomycin, Fungizone ${ }^{\circledR}(0.5 \mu \mathrm{g}$ amphotericin $\mathrm{B}, 0.41 \mu \mathrm{g} / \mathrm{mL}$ sodium deoxycholate), and $1 \mathrm{mM}$ sodium pyruvate. H441 and Calu-3 cells were maintained in RPMI and MEM media, respectively, with the same additives as DMEM.

\subsubsection{Cellular uptake}

To observe inherent cellular uptake of MP systems, A549, H441, and Calu-3 cells were incubated with MP and observed using a Cytation 3 image reader (BioTek, Winooski, VT, USA). Cells (5,000 cells/well) were seeded in a 24-well plate and incubated overnight at $37^{\circ} \mathrm{C}$. Cells were incubated with MP containing $10 \mu \mathrm{M}$ SRB for 3 hours. After incubation, media was removed, and the cells were washed twice with 1x PBS to remove any unbound MP. To visualize cellular uptake, images were captured using fluorescence imaging. For quantification of cellular uptake, the

presence of the SRB within the cells was analyzed via fluorescence spectroscopy at $565 / 586 \mathrm{~nm}(\mathrm{ex} / \mathrm{em})$.

\subsubsection{In vitro cytotoxicity assay}

The cytotoxic effect of the formulated MP on A549 cells was determined. Cells were seeded in 96-well plate at 5,000 cells/well and incubated overnight. The following day, cells were exposed to varying concentrations of SRB (0.01 to $10 \mu \mathrm{M})$ 
from formulated MP and cytotoxicity was assessed. $60 \mu \mathrm{M}$ resazurin was added to the cells and incubated for 3 hours. The fluorescence intensity of resorufin produced by viable cells was detected at $544 \mathrm{~nm}$ (excitation) and $590 \mathrm{~nm}$ (emission). The relative viability of each sample was calculated by:

$$
\text { Relative Viability }=\frac{\text { Sample Fluorescence Intensity }}{\text { Control Fluorescence Intensity }} \times 100 \%
$$

4.5.13 In vitro transepithelial electrical resistance of lung epithelial cells exposed to $M P$

H441 cells were used for transepithelial electrical resistant (TEER) analysis following microparticle exposure. Cells were seeded at $1 \times 10^{5} \mathrm{cells} / \mathrm{ml}$ in well plates with $12 \mathrm{~mm}$ Transwells ${ }^{\circledR}$ having $0.4 \mu \mathrm{m}$ pore size (Corning Incorporated, Corning, $\mathrm{NY}$, USA) with $1.5 \mathrm{~mL}$ of media on the basolateral side and $0.5 \mathrm{~mL}$ of media on the apical side. On day 3, the cells were transitioned to air-interface conditions (AIC) with $0.5 \mathrm{~mL}$ of media on the basolateral side and no media on the apical side. Prior to TEER measurements, $0.5 \mathrm{ml}$ of media was added to the apical side of the Transwell and the resistance was measured using an EVOM voltohmmeter with an STX2 electrode (World Precision Instruments, INC., Sarasota, FL, USA). Once the resistance stabilized to $100 \Omega \mathrm{cm}^{2}$, indicating a confluent monolayer, the cells were exposed to $1 \mathrm{mg}$ of the microparticles. Resistance measurements were taken for five days after exposure and the TEER of cells exposed to particles was compared to the control cells to calculate the percent TEER response as follows: 


$$
\text { TEER } \% \text { Control }=\frac{\text { Sample resistance }}{\text { Control resistance }} \times 100 \%
$$

\subsubsection{Statistical analysis}

All measurements were performed in at least triplicate. Statistical significance for in vitro cellular studies was determined using two-way ANOVA with Tukey's multiple comparisons test (GraphPad Prism version 7). A p-value of $<0.05$ was considered as statistically significant. Values were presented as mean \pm standard deviation unless stated otherwise.

\subsection{Results}

4.6.1 Confirmation of acetalated dextran (Ac-Dex) synthesis and microparticle morphology

Fast-Ac-Dex exhibited a cyclic acetal coverage (CAC) ratio of $43.5 \%$ and $-\mathrm{OH}$ conversion of $75.1 \%$, while Slow-Ac-Dex exhibited an $80.6 \%$ CAC ratio and 93.8\% $\mathrm{OH}$ conversion. As seen in Figure 4.2, Ac-Dex MP systems exhibited corrugated (raisin-like) morphology, whereas mannitol MP exhibited spherical morphology. 


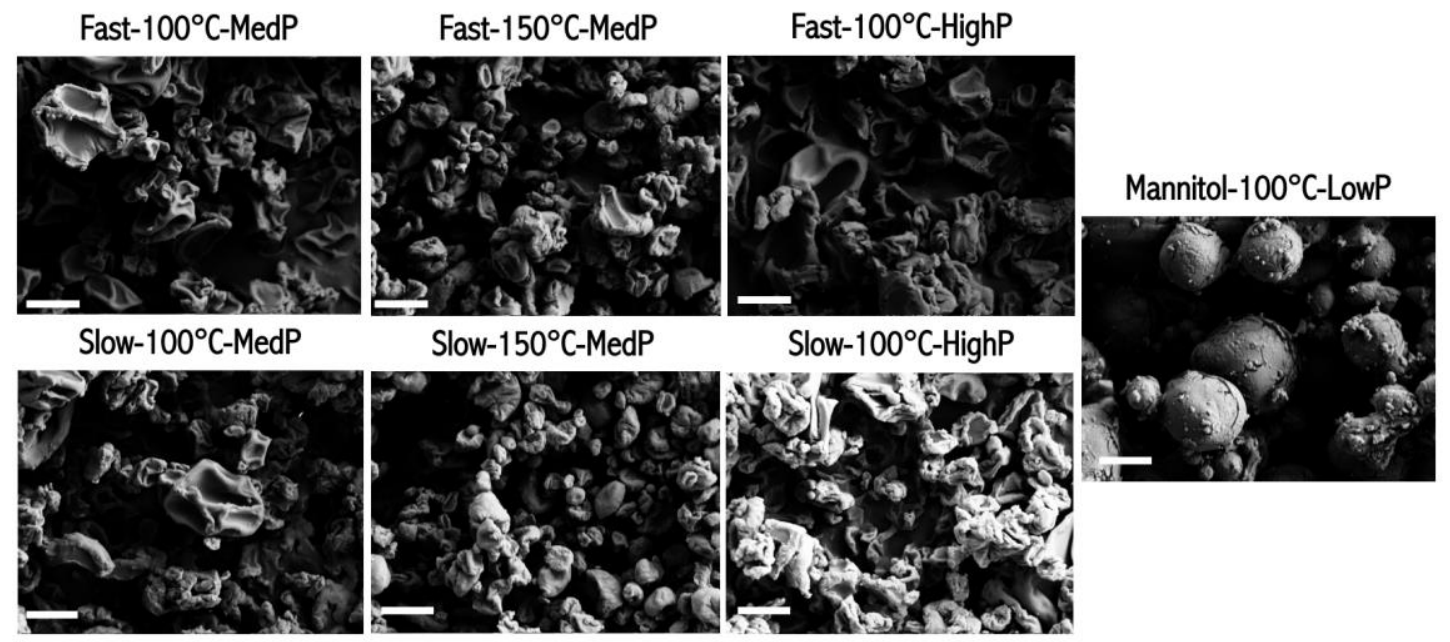

Figure 4.2. Representative scanning electron microscopy micrographs of spray-dried sulforhodamine B (SRB)-loaded microparticles. Images were taken at 10,000x. Scale

$$
\mathrm{bar}=2 \mu \mathrm{m}
$$

\subsubsection{Size analysis and in vitro aerosol performance}

Microparticles were analyzed for their size and density to aid in the understanding of particle performance (Table 4.2). Hydrodynamic diameters for AcDex MP ranged from 0.96 to $1.63 \mu \mathrm{m}$ whereas PDI values ranged from 0.16 to 0.30 . Tapped density values ranged from 0.117 to $0.157 \mathrm{~g} / \mathrm{cm}^{3}$ and the resulting theoretical MMAD values were between 0.20 and $0.41 \mu \mathrm{m}$. MP systems deposited on all stages of the impactor (Figure 4.3), with the largest amounts depositing on stages 2 through 5. Experimental MMAD values for the MP ranged from 2.1 to $2.9 \mu \mathrm{m}$, while the geometric standard deviations ranged from 1.9 to $2.7 \mu \mathrm{m}$. In comparison mannitol MP exhibited similar hydrodynamic diameter, density, and GSD and higher PDI, MMADT $_{\mathrm{T}}$, and MMADEXP than Ac-Dex MP. Fine particle fraction (FPF), respirable fraction (RF), and emitted dose (ED) values are reported in Figures 4.3 and B1, 
representing the aerosol performance properties of the inhaled dry powders. FPF were $>90 \%$, RF ranged from 65 to $78 \%$, and ED were $>88 \%$.

Table 4.2. Characteristics of microparticle systems, including (Top) hydrodynamic diameter and polydispersity index (PDI) via dynamic light scattering, tapped density, theoretical mass median aerodynamic diameter $\left(\mathrm{MMAD}_{\mathrm{T}}\right)$ via tapped density calculations, and experimental mass median aerodynamic diameter (MMAD $\mathrm{EXP}_{\text {) }}$ and geometric standard deviation (GSD) via Next Generation Impactor analysis and (Bottom) water content, encapsulation efficiency, and drug loading of sulforhodamine $\mathrm{B}(\mathrm{SRB})$ in the microparticles (mean \pm standard deviation, $\mathrm{n}=3$ ).

\begin{tabular}{|c|c|c|c|c|c|c|c|}
\hline System & $\begin{array}{l}\text { Hydrodynamic } \\
\text { Diameter }(\mu \mathrm{m})\end{array}$ & PDI & $\begin{array}{c}\text { Tapped } \\
\text { Density } \\
\left(\mathrm{g} / \mathrm{cm}^{3}\right)\end{array}$ & $\underset{(\mu \mathrm{m})}{\text { MMAD }_{\mathrm{T}}}$ & $\begin{array}{c}\text { MMADE }_{\mathrm{XP}}(\boldsymbol{\mu m})\end{array}$ & $\begin{array}{c}\text { Geometr } \\
\text { ic } \\
\text { diamete } \\
\mathbf{r}\left(\mathbf{d}_{\mathrm{g}}\right) \\
(\boldsymbol{\mu \mathrm { m }}) \\
\end{array}$ & GSD \\
\hline $\begin{array}{c}\text { Fast- } \\
100^{\circ} \mathrm{C}- \\
\text { MedP }\end{array}$ & $1.01 \pm 0.27$ & $\begin{array}{c}0.24 \pm \\
0.03\end{array}$ & $\begin{array}{c}0.139 \pm \\
0.002\end{array}$ & $\begin{array}{c}0.39 \pm \\
0.19\end{array}$ & $\begin{array}{c}2.60 \pm \\
0.30\end{array}$ & $\begin{array}{l}1.13 \pm \\
0.56\end{array}$ & $\begin{array}{c}2.66 \pm \\
1.53\end{array}$ \\
\hline $\begin{array}{l}\text { Fast- } \\
150^{\circ} \mathrm{C}- \\
\text { MedP }\end{array}$ & $0.98 \pm 0.03$ & $\begin{array}{c}0.29 \pm \\
0.03\end{array}$ & $\begin{array}{c}0.157 \pm \\
0.016\end{array}$ & $\begin{array}{c}0.20 \pm \\
0.07\end{array}$ & $\begin{array}{c}2.06 \pm \\
0.25\end{array}$ & $\begin{array}{c}0.53 \pm \\
0.19\end{array}$ & $\begin{array}{c}1.86 \pm \\
0.13\end{array}$ \\
\hline $\begin{array}{c}\text { Fast- } \\
100^{\circ} \mathrm{C}- \\
\text { HighP }\end{array}$ & $1.57 \pm 0.06$ & $\begin{array}{c}0.60 \pm \\
0.19\end{array}$ & $\begin{array}{c}0.130 \pm \\
0.004\end{array}$ & $\begin{array}{c}0.41 \pm \\
0.17\end{array}$ & $\begin{array}{c}2.78 \pm \\
0.30\end{array}$ & $\begin{array}{c}1.11 \pm \\
0.45\end{array}$ & $\begin{array}{c}2.05 \pm \\
0.17\end{array}$ \\
\hline $\begin{array}{l}\text { Slow- } \\
100^{\circ} \mathrm{C}- \\
\text { MedP }\end{array}$ & $1.18 \pm 0.03$ & $\begin{array}{c}0.22 \pm \\
0.01\end{array}$ & $\begin{array}{c}0.135 \pm \\
0.003\end{array}$ & $\begin{array}{c}0.41 \pm \\
0.18\end{array}$ & $\begin{array}{c}2.29 \pm \\
0.29\end{array}$ & $\begin{array}{c}1.15 \pm \\
0.51\end{array}$ & $\begin{array}{c}1.93 \pm \\
0.06\end{array}$ \\
\hline $\begin{array}{l}\text { Slow- } \\
150^{\circ} \mathrm{C}- \\
\text { MedP }\end{array}$ & $0.96 \pm 0.03$ & $\begin{array}{c}0.16 \pm \\
0.03\end{array}$ & $\begin{array}{c}0.117 \pm \\
0.003\end{array}$ & $\begin{array}{c}0.40 \pm \\
0.20\end{array}$ & $\begin{array}{c}2.61 \pm \\
0.45\end{array}$ & $\begin{array}{c}1.01 \pm \\
0.51\end{array}$ & $\begin{array}{c}2.00 \pm \\
0.26\end{array}$ \\
\hline $\begin{array}{l}\text { Slow- } \\
100^{\circ} \mathrm{C}- \\
\text { HighP }\end{array}$ & $1.63 \pm 0.08$ & $\begin{array}{c}0.39 \pm \\
0.37\end{array}$ & $\begin{array}{c}0.131 \pm \\
0.006\end{array}$ & $\begin{array}{c}0.20 \pm \\
0.09\end{array}$ & $\begin{array}{c}2.86 \pm \\
0.23\end{array}$ & $\begin{array}{l}0.556 \pm \\
0.24\end{array}$ & $\begin{array}{c}1.96 \pm \\
0.22\end{array}$ \\
\hline $\begin{array}{c}\text { Man- } \\
100^{\circ} \mathrm{C}- \\
\text { LowP }\end{array}$ & $1.48 \pm 0.10$ & $\begin{array}{c}0.74 \pm \\
0.02\end{array}$ & $\begin{array}{c}0.129 \pm \\
0.003\end{array}$ & $\begin{array}{c}1.05 \pm \\
0.67\end{array}$ & $\begin{array}{c}3.73 \pm \\
0.80\end{array}$ & $\begin{array}{c}3.53 \pm \\
2.3\end{array}$ & $\begin{array}{c}1.92 \pm \\
0.21\end{array}$ \\
\hline \multicolumn{2}{|c|}{ System } & $\begin{array}{c}\text { Water Content } \\
(\%)\end{array}$ & \multicolumn{2}{|c|}{$\begin{array}{l}\text { SRB Encapsulation } \\
\text { Efficiency }(\%)\end{array}$} & \multicolumn{3}{|c|}{$\begin{array}{c}\text { Drug Loading } \\
(\mu \mathrm{g} \text { SRB/mg particles) }\end{array}$} \\
\hline \multicolumn{2}{|c|}{ Fast- $100^{\circ} \mathrm{C}-\mathrm{MedP}$} & $2.2 \pm 0.7$ & \multicolumn{2}{|c|}{$153 \pm 45$} & \multicolumn{3}{|c|}{$15.2 \pm 4.5$} \\
\hline
\end{tabular}




\begin{tabular}{|c|c|c|c|}
\hline Fast- $150^{\circ} \mathrm{C}-\mathrm{MedP}$ & $3.2 \pm 0.7$ & $165 \pm 53$ & $16.1 \pm 4.8$ \\
\hline Fast- $100^{\circ} \mathrm{C}-\mathrm{HighP}$ & $1.9 \pm 0.2$ & $148 \pm 27$ & $15.7 \pm 4.5$ \\
\hline Slow- $100^{\circ} \mathrm{C}-\mathrm{MedP}$ & $3.2 \pm 0.7$ & $147 \pm 29$ & $14.7 \pm 2.9$ \\
\hline Slow- $150^{\circ} \mathrm{C}-\mathrm{MedP}$ & $2.9 \pm 1.5$ & $142 \pm 36$ & $14.0 \pm 3.5$ \\
\hline Slow- $100^{\circ} \mathrm{C}-\mathrm{HighP}$ & $3.5 \pm 1.1$ & $129 \pm 29$ & $16.7 \pm 2.9$ \\
\hline Man- $100^{\circ} \mathrm{C}-$ LowP & $2.8 \pm 1.4$ & $118 \pm 27$ & $11.6 \pm 2.7$ \\
\hline
\end{tabular}
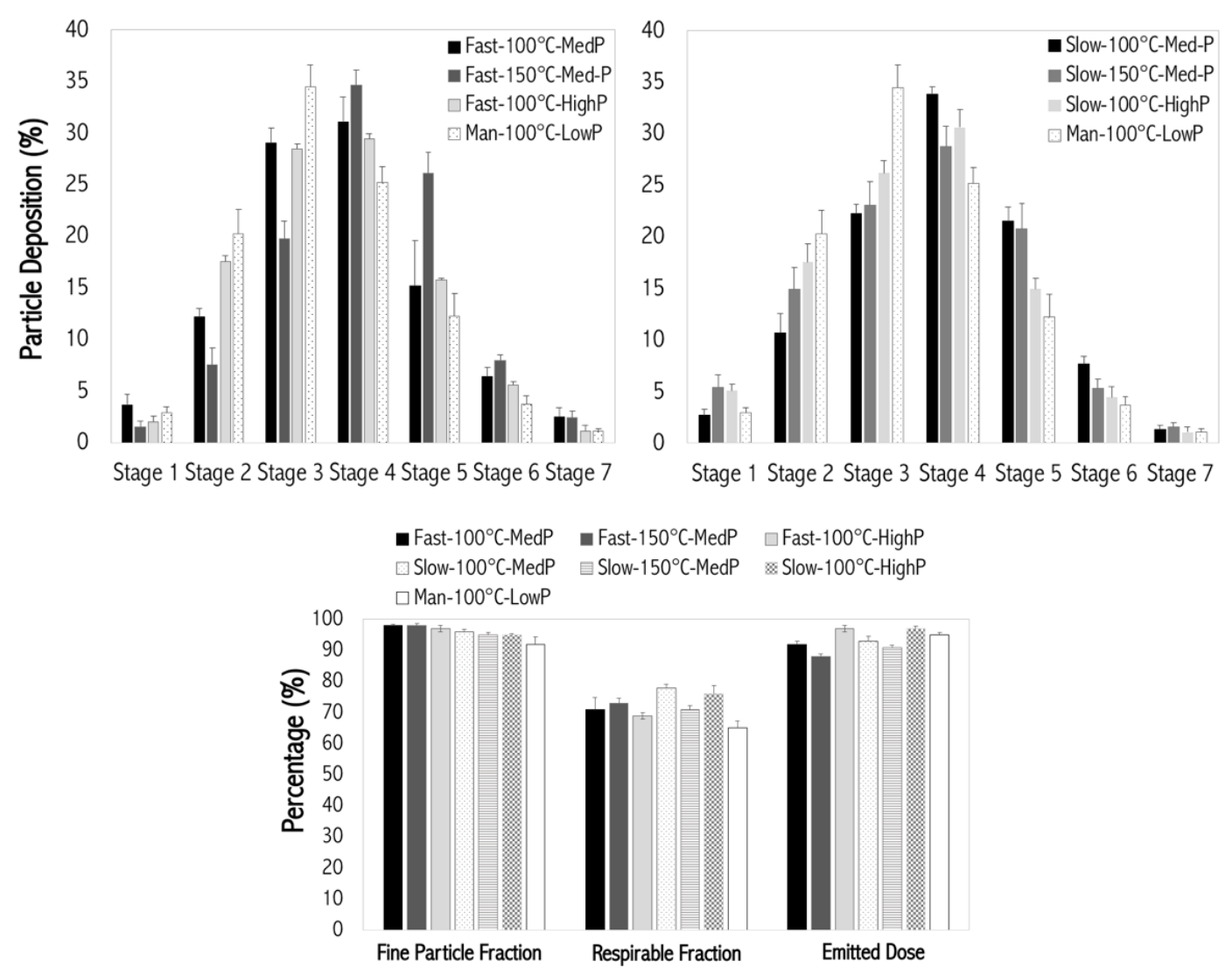

Figure 4.3. (Top) Aerosol dispersion analysis of fast and slow degrading particles in stages 1-7 of a Next Generation Impactor, expressed as percent particle deposition per stage (mean \pm standard deviation, $n=3$ ). For $Q=60 \mathrm{~L} / \mathrm{min}$, cutoffs for each stage of the NGI are: $8.06 \mu \mathrm{m}$ (stage 1), $4.46 \mu \mathrm{m}$ (stage 2), $2.82 \mu \mathrm{m}$ (stage 3), $1.66 \mu \mathrm{m}$ (stage 4), $0.94 \mu \mathrm{m}$ (stage 5), $0.55 \mu \mathrm{m}$ (stage 6) and $0.34 \mu \mathrm{m}$ (stage 7). (Bottom) Aerosolization performance properties of spray-dried particle systems, including fine 
particle fraction (FPF), respirable fraction (RF), and emitted dose (ED) (mean \pm standard deviation, $\mathrm{n}=3$ ).

\subsubsection{Solid state analysis}

MP systems exhibited water content values $\leq 3.5 \%$ (Table 4.2) and DSC thermograms (Figure 4.2A) indicate that the formulations exhibited thermal transitions above $90^{\circ} \mathrm{C}$ (shown by arrows), which is greater than the spray dryer outlet temperatures (Table 4.1, e.g. temperatures that the particles were exposed to during spray drying). XRD diffractograms (Figure 4.2B) indicate the transition of SRB from a crystalline to amorphous state when spray-dried and encapsulated in Ac-Dex, and a crystalline state following encapsulation in mannitol, which is similar to previous reports $(53,54)$. Also, characteristic peaks of SRB were present in the mannitol formulation (Figure 4.2B, as indicated by arrows). Absence of diffraction in Fast-AcDex suggest minimal to no crystallinity of the material (44). For confirmation, PLM images were taken of the raw materials and MP systems (Figure 4.2C). Raw mannitol, raw SRB, and a mixture of the two are visibly crystalline, as indicated by the high level of birefringency. Images show minimal birefringency for Fast- $150^{\circ} \mathrm{C}$ MedP and Slow- $100^{\circ} \mathrm{C}-\mathrm{HighP}$, whereas other systems appear to have none. 

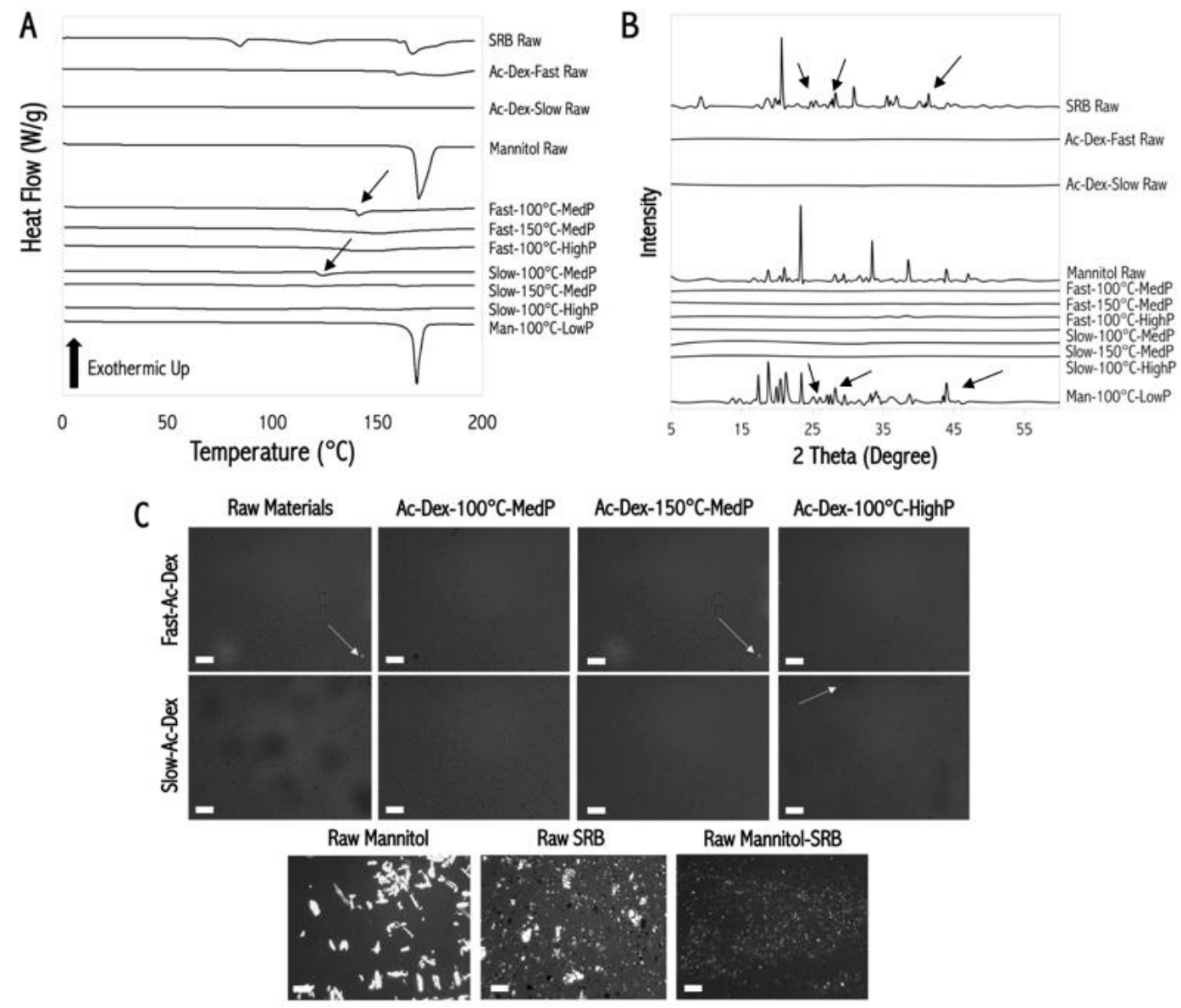

Figure 4.4. (A) Representative differential scanning calorimetry thermograms of raw materials used during the spray drying of particles and of sulforhodamine B (SRB)loaded microparticles. (B) Representative x-ray diffractograms of raw materials used during the spray drying of particles and of sulforhodamine B (SRB)-loaded microparticles. (C) Polarized light microscopy images of microparticle systems and raw materials. Crystals in microparticle systems are indicated by arrows, if applicable.

Scale bar $=0.1 \mathrm{~mm}$. 


\subsubsection{Drug loading, in vitro drug release, and release kinetic analysis}

All particles exhibited SRB encapsulation efficiency (EE) values greater than 100\% (Table 4.2), whereas SRB drug loading (DL) ranged from 11.6 to $16.1 \mu \mathrm{g}$ of SRB to mg of MP. The exceptionally high EE is mainly based on the loss of polymer during synthesis; smaller particles are captured by the filter rather than the collection

vial and cyclone. This polymer loss results in an inherent increase in EE. Although drug loading values were exceptionally low, for the purposes of the study, they were determined to be acceptable.

The in vitro drug release of SRB from all MP systems in phosphate buffer $(\mathrm{pH}$ 7.4) and sodium acetate buffer ( $\mathrm{pH}$ 5.35) at physiological temperature was performed to simulate release in circulation and acidic states of the body, respectively (Figure 4.5). Results show that both types of Ac-Dex MP systems released 100\% of SRB between 4 and 6 hours in acidic $\mathrm{pH}$, which is characteristic of the acid-sensitive nature of Ac-Dex $(19,36)$. Although all Fast-Ac-Dex MP systems except for Fast- $100^{\circ} \mathrm{C}$ MedP reach equilibrium at 36 hours, at physiological pH, Fast-Ac-Dex MP released $100 \%$ of SRB at 96 hours. In comparison, Slow-Ac-Dex MP released 100\% of SRB at 108 hours. Mannitol MP released 100\% of SRB in 4 hours at both $\mathrm{pH}$ values (while reaching equilibrium in the first 2 hours), corresponding to its high solubility in aqueous environments and lack of $\mathrm{pH}$ sensitivity.

To investigate the theoretical outcome of the release of SRB from the designed systems and their subsequent release mechanism(s), the release profiles were fitted to several models, including zero-order, first-order, Higuchi, Korsmeyer-Peppas, PeppasSahlin, and Corrigan. Table 4.3 shows the results of the best-fit models at each $\mathrm{pH}$, 
their associated constants, and their $\mathrm{R}^{2}$ values (all of which were $\geq 0.90$ ). At $\mathrm{pH} 5.35$, Ac-Dex MP exhibited both Corrigan and Peppas-Sahlin-type release kinetics. The fraction of drug release $\left(\mathrm{F}_{\mathrm{B}, \text { in }}\right)$ show that the majority of the drug cargo was released and the $\mathrm{K}_{\mathrm{C}}$ values varied from system-to-system (227). Drug release kinetics at $\mathrm{pH} 7.4$ for Fast-Ac-Dex and mannitol MP were best fit to the Korsmeyer-Peppas model, which relates the amount of drug released to the time elapsed (228). The $n$ variable in the model determines the type of diffusion from the formulation. Results show that particle systems exhibiting Korsmeyer-Peppas release kinetics have $\mathrm{n}<0.43$, indicating that the release from the MP followed Fickian diffusion (229). Slow-AcDex MP exhibited first-order release kinetics at $\mathrm{pH} 7.4$ and the values of $\mathrm{K}_{1}$ for SlowAc-Dex systems are considerably smaller in relation to the values of $t$ and the constant, n. The values from this study show that Slow-Ac-Dex systems exhibited short burst release followed by sustained release (2). 

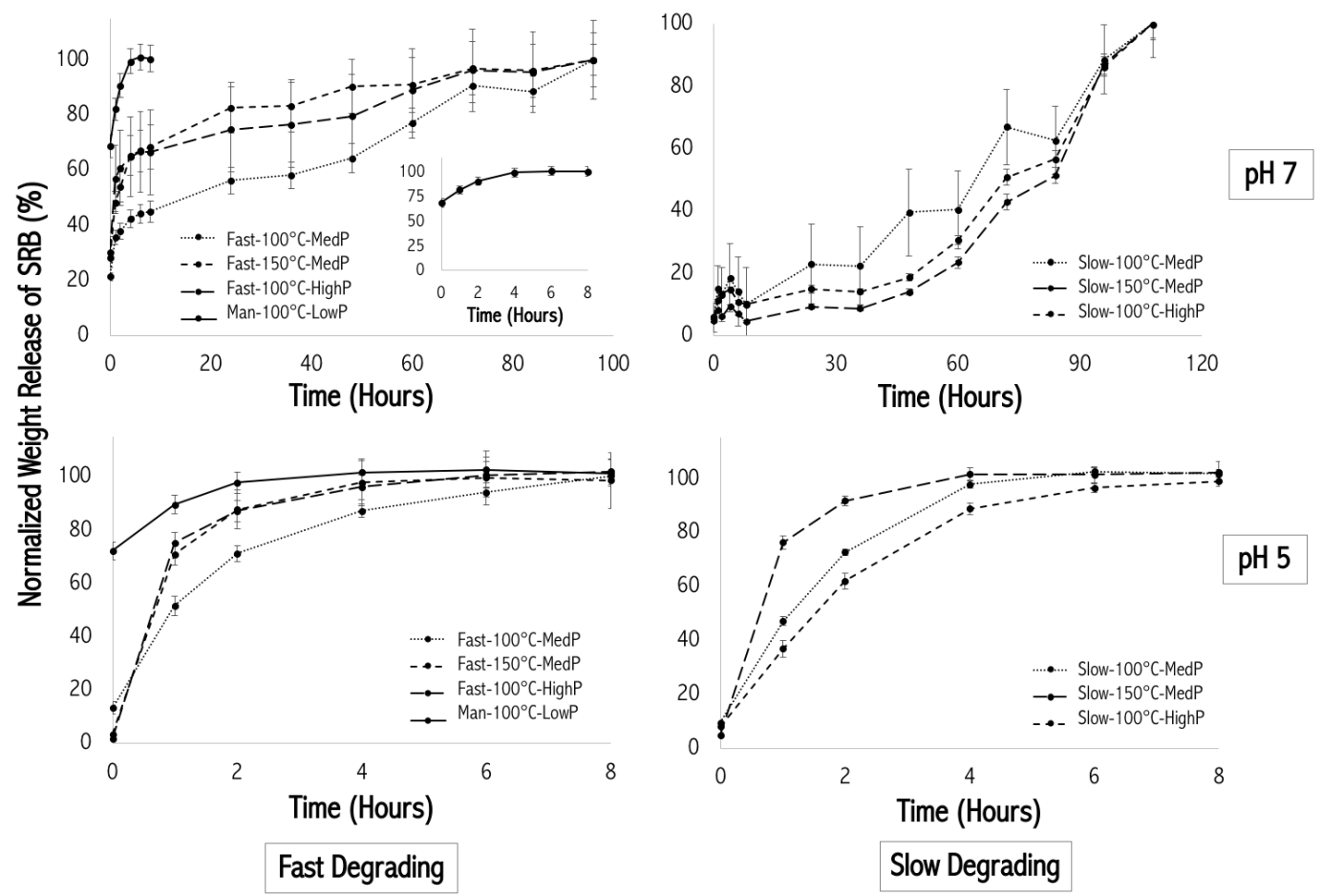

Figure 4.5. Normalized percent weight release profiles of sulforhodamine-B (SRB) microparticles at $\mathrm{pH} 5$ and $\mathrm{pH} 7$ as a function of time (mean \pm standard deviation, $\mathrm{n}=$ 3). The insert for $\mathrm{pH} 7$, fast degrading particles represents the zoomed in release for mannitol microparticles. 
Table 4.3 Best-fit drug release kinetics model for each microparticle system, the corresponding fitted parameters, and corresponding coefficient of determination $\left(\mathrm{R}^{2}\right)$ values. $\mathrm{K}_{\mathrm{KP}}$ is the Korsmeyer release rate constant, $\mathrm{n}$ is the drug release exponent, $\mathrm{K}_{1}$ is the first-order release rate constant, $\mathrm{K}_{\mathrm{C}}$ is the first-order rate constant associated with kinetics of the burst release for Corrigan, $\mathrm{F}_{\mathrm{B} \text {,in }}$ is the fraction of drug release during burst. The units for the constants are as follows: $K_{K P}[=] h^{-n}, K_{1}[=] h^{-1}, n[=]$ unitless, $\mathrm{K}_{\mathrm{C}}[=] \mathrm{h}^{-1}, \mathrm{~F}_{\mathrm{B}, \text { in }}[=]$ unitless (fraction), $\mathrm{k}_{1}[=] \mathrm{h}^{-\mathrm{m}}, \mathrm{k}_{2}[=] \mathrm{h}^{-2 \mathrm{~m}}, \mathrm{~m}[=]$ unitless.

\begin{tabular}{|c|c|c|c|c|c|}
\hline \multicolumn{6}{|c|}{$\mathrm{pH}=5.35$} \\
\hline System & Model & \multicolumn{3}{|c|}{ Constants } & $\mathbf{R}^{2}$ \\
\hline Fast $100^{\circ} \mathrm{C}-\mathrm{MedP}$ & Corrigan & \multicolumn{2}{|c|}{$\mathrm{K}_{\mathrm{C}}=0.673$} & $\mathrm{~F}_{\mathrm{B}, \text { in }}=0.979$ & 0.964 \\
\hline Fast- $150^{\circ} \mathrm{C}-\mathrm{MedP}$ & Corrigan & \multicolumn{2}{|c|}{$\mathrm{K}_{\mathrm{C}}=1.211$} & $\mathrm{~F}_{\mathrm{B}, \mathrm{in}}=0.989$ & 0.997 \\
\hline Fast- $100^{\circ} \mathrm{C}$-HighP & Corrigan & \multicolumn{2}{|c|}{$\mathrm{K}_{\mathrm{C}}=1.306$} & $\mathrm{~F}_{\mathrm{B}, \mathrm{in}}=0.992$ & 0.993 \\
\hline Slow-100 ${ }^{\circ} \mathrm{C}-\mathrm{MedP}$ & Corrigan & \multicolumn{2}{|c|}{$\mathrm{K}_{\mathrm{C}}=0.632$} & $\mathrm{~F}_{\mathrm{B}, \mathrm{in}}=1.030$ & 0.985 \\
\hline Slow-150 C-MedP & Corrigan & \multicolumn{2}{|c|}{$\mathrm{K}_{\mathrm{C}}=0.481$} & $\mathrm{~F}_{\mathrm{B}, \mathrm{in}}=1.014$ & 0.990 \\
\hline Slow- $100^{\circ} \mathrm{C}$-HighP & \multirow{2}{*}{ Corrigan } & \multicolumn{2}{|c|}{$\mathrm{K}_{\mathrm{C}}=1.358$} & $\mathrm{~F}_{\mathrm{B}, \mathrm{in}}=1.010$ & 0.995 \\
\hline Man- $100^{\circ} \mathrm{C}$-LowP & & & -- & --- & --- \\
\hline \multicolumn{6}{|c|}{$\mathrm{pH}=5.35$} \\
\hline System & Model & \multicolumn{3}{|c|}{ Constants } & $\mathbf{R}^{2}$ \\
\hline Fast- $100^{\circ} \mathrm{C}-\mathrm{MedP}$ & Peppas-Sahlin & \multirow{2}{*}{$\begin{array}{l}\mathrm{k}_{1}=0.6304 \\
\mathrm{k}_{1}=0.6926\end{array}$} & $\mathrm{k}_{2}=0.1524$ & $\mathrm{~m}=0.0912$ & 0.969 \\
\hline Fast- $150^{\circ} \mathrm{C}-\mathrm{MedP}$ & Peppas-Sahlin & & $\mathrm{k}_{2}=0.1130$ & $\mathrm{~m}=0.0763$ & 0.969 \\
\hline Fast- $100^{\circ} \mathrm{C}$-HighP & Peppas-Sahlin & $\mathrm{k}_{1}=0.6930$ & $\mathrm{k}_{2}=0.1298$ & $\mathrm{~m}=0.0699$ & 0.979 \\
\hline Slow- $100^{\circ} \mathrm{C}$-MedP & Peppas-Sahlin & $\mathrm{k}_{1}=0.6132$ & $\mathrm{k}_{2}=0.1922$ & $\mathrm{~m}=0.0643$ & 0.970 \\
\hline Slow-150 ${ }^{\circ} \mathrm{C}-\mathrm{MedP}$ & Peppas-Sahlin & $\mathrm{k}_{1}=0.4767$ & $\mathrm{k}_{2}=0.2894$ & $\mathrm{~m}=0.0581$ & 0.978 \\
\hline Slow- $100^{\circ} \mathrm{C}-\mathrm{HighP}$ & Peppas-Sahlin & $\mathrm{k}_{1}=0.6912$ & $\mathrm{k}_{2}=0.2520$ & $\mathrm{~m}=0.0237$ & 0.967 \\
\hline Man- $100^{\circ} \mathrm{C}$-LowP & Peppas-Sahlin & --- & --- & --- & --- \\
\hline \multicolumn{6}{|c|}{$\mathrm{pH}=7.4$} \\
\hline System & Model & \multicolumn{2}{|c|}{ Constants } & \multicolumn{2}{|l|}{$\mathbf{R}^{2}$} \\
\hline Fast- $100^{\circ} \mathrm{C}-\mathrm{MedP}$ & Korsmeyer-Peppas & $\mathrm{K}_{\mathrm{KP}}=0.314$ & $\mathrm{n}=0.218$ & \multicolumn{2}{|c|}{0.907} \\
\hline Fast- $150^{\circ} \mathrm{C}-\mathrm{MedP}$ & Korsmeyer-Peppas & $\mathrm{K}_{\mathrm{KP}}=0.546$ & $\mathrm{n}=0.117$ & \multicolumn{2}{|c|}{0.921} \\
\hline Fast- $100^{\circ} \mathrm{C}$-HighP & Korsmeyer-Peppas & $\mathrm{K}_{\mathrm{KP}}=0.498$ & $\mathrm{n}=0.152$ & \multicolumn{2}{|c|}{0.987} \\
\hline Slow- $100^{\circ} \mathrm{C}-\mathrm{MedP}$ & First Order & $\mathrm{K}_{1}=0.0248$ & --- & \multicolumn{2}{|c|}{0.942} \\
\hline Slow- $150^{\circ} \mathrm{C}-\mathrm{MedP}$ & First Order & $\mathrm{K}_{1}=0.0267$ & --- & 0.97 & \\
\hline Slow- $100^{\circ} \mathrm{C}-\mathrm{HighP}$ & First Order & $\mathrm{K}_{1}=0.0272$ & -- & 0.90 & \\
\hline Man- $100^{\circ} \mathrm{C}$-LowP & Korsmeyer-Peppas & $\mathrm{K}_{\mathrm{KP}}=0.831$ & $\mathrm{n}=0.103$ & 0.92 & \\
\hline
\end{tabular}




\subsubsection{Cytotoxicity, uptake, and transepithelial electrical resistance of cells}

The integrity of H441 air-interface culture (AIC) lung epithelial cells exposed to aerosolized MP was analyzed using transepithelial electrical resistance (TEER). The presence of an initial confluent monolayer was confirmed by steady TEER values (> $100 \Omega \mathrm{cm}^{2}$ ) following 9-11 days of culturing in AIC conditions. Beginning 24 hours after exposure to the formulated particles, TEER was evaluated. As seen in Figure 4.6A, the TEER values remained statistically the same or higher following MP exposure $(\mathrm{p}>0.05)$ and were similar to that of the control. The influence of the formulated MP systems on A549 viability was evaluated (Figure 4.6B) and results indicate that the cells exposed to MP exhibited viability values similar to those the control for up to 108 hours of exposure. Increasing the concentration of microparticles imparted no significant change in the viability of A549 cells for the concentrations tested. The cellular uptake of the MP systems was evaluated in A549, Calu-3, and H441 cells for 3 hours (Figure 4.6C). The presence of SRB-loaded MP within the cells were detected via fluorescence imaging and spectroscopy. Significantly more Fast-Ac-Dex MP were uptaken by all the cells in comparison to Slow-Ac-Dex MP. In addition, more MP uptake was evident in Calu-3 and H441 cells in comparison to A549 cells. The morphology of the cells was similar at all time points and following exposure to the MP. 


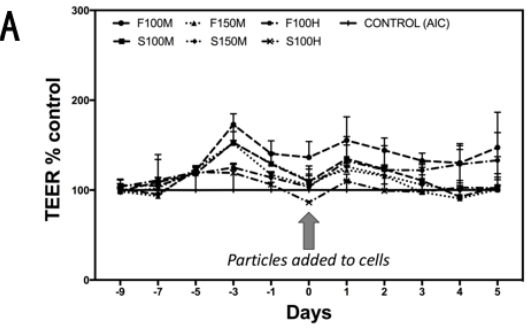

B
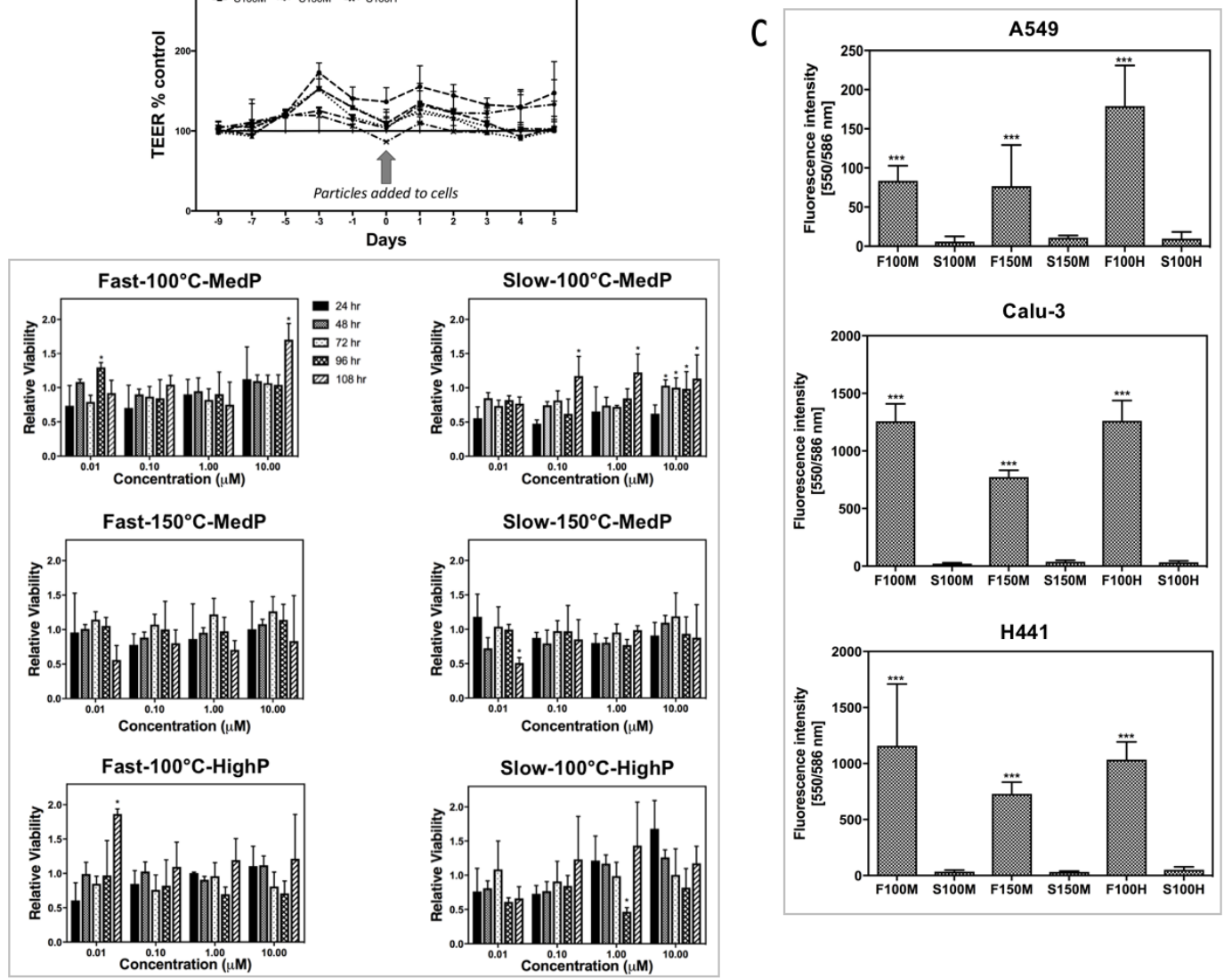

Figure 4.6. Results from experiments where cells were exposed to sulforhodamine B (SRB)-loaded microparticles (MP), including (A) Transepithelial electrical resistance (TEER) data for H441 cells exposed to microparticle systems from day -9 to day 5, where day 0 was the particle exposure day. The particle naming convention corresponds to the following: $\mathrm{F} 100 \mathrm{M}=$ Fast $-100^{\circ} \mathrm{C}-\mathrm{MedP}, \mathrm{S} 100 \mathrm{M}=\mathrm{Slow}-100^{\circ} \mathrm{C}-$ MedP, F150M $=$ Fast $-150^{\circ} \mathrm{C}-\mathrm{MedP}, \mathrm{S} 150 \mathrm{M}=\mathrm{Slow}-150^{\circ} \mathrm{C}-\mathrm{MedP}, \mathrm{F} 100 \mathrm{H}=$ Fast $100^{\circ} \mathrm{C}$-HighP, and $\mathrm{S} 100 \mathrm{H}=\mathrm{Slow}-100^{\circ} \mathrm{C}-\mathrm{HighP}$; (B) Viability of A549 cells exposed to SRB MP $\left(* \mathrm{p}<0.05,{ }^{* *} \mathrm{p}<0.01\right.$, mean \pm standard deviation, $\left.\mathrm{n}=3\right)$; and $(\mathrm{C}) \mathrm{SRB}$ MP uptake by A549, Calu-3, and H441 cell lines (* p < 0.05, **p $<0.01$, ***p $<$ 0.001, mean \pm standard deviation, $\mathrm{n}=3$ ). 


\subsection{Discussion}

Ac-Dex is a biodegradable polymer that exhibits tunable degradation rates based on the synthesis time and formation and type of acetals (cyclic versus acyclic). A higher $\mathrm{CAC}$ ratio results in slower degradation, whereas higher $-\mathrm{OH}$ conversion results in Ac-Dex that is more hydrophobic, allowing for facile particle formation. For the described formulations, Fast-Ac-Dex data were similar to previously reported data $(19,40,43,45,54)$ and Slow-Ac-Dex values were similar or higher than previous reports $(45,49)$. Furthermore, the increase in CAC for Slow-Ac-Dex correlates to slower release, as shown in the kinetic release analysis.

Differences in the morphology of the MP systems (Ac-Dex MP = corrugated, mannitol $\mathrm{MP}=$ spherical) are due to the difference in surface tensions and boiling points of the solvents used during spray drying. For Ac-Dex MP, solvent droplets comprised primarily of IPA (and Ac-Dex) and a small amount water (and SRB) are vaporized rapidly due to the high inlet temperatures $\left(\geq 100^{\circ} \mathrm{C}\right)$ in relation to the boiling point of IPA $\left(82.6^{\circ} \mathrm{C}\right)$. Owing to the low surface tension of IPA and fast solvent vaporization during spray drying, the droplets dry quickly and a thin polymer wall forms, leading to hollow particles. As the particles cool, the walls of the droplets collapse, resulting in a corrugated structure $(45,145)$. In contrast, the spray-dried mannitol solution contains only mannitol and SRB in water. Due to the high surface tension and boiling point of water, these droplets are able to hold their spherical shape more efficiently during the drying process, allowing for the formation of spherical particles (145). 
The analysis of particle hydrodynamic diameters in solution allow for the observation of MP in their wet state, and PDI values indicate that the particles were fairly homogenous in size in this state. The tapped densities of the MP systems were much lower than the reference density $\left(1 \mathrm{~g} / \mathrm{cm}^{3}\right)$. For Ac-Dex MP, low density values are likely due to the corrugated morphology of the particles and the void space of both particle types in their dry state can lower the overall density values. The low tapped density values resulted in theoretical MMAD values that are smaller than the geometric diameters, indicating that the actual densities of the MP are higher than reported here.

Experimental MMAD and GSD values were similar to previously reported data (45). Theoretical MMAD of all particle sets were less than MMADEXP, which is likely due to the agglomeration of the dry particles during aerosolization (45). MMADECP values were within the $1-5 \mu \mathrm{m}$ range required for aerosol deposition of particles into the lungs (135). FPF values $>90 \%$ indicate that the aerosolized particles have the potential to deposit in the lower airways. High ED values indicate that the majority of particles are released from the capsules. In addition, a significant portion of particles have the potential to be delivered from the inhaler to the lower airways of the lungs, as indicated by high RF values. In all cases, these values were higher in comparison to previously published reports $(45,218,230-232)$. Particles that were synthesized using a high pump rate exhibited higher PDI, hydrodynamic diameters, and experimental MMAD values. This is likely due to the lower exposure time to heated gas due to the higher pump rate. Solutions dried using these settings allow for more rapid drying in comparison to other settings, which allows for uneven formation 
of particles. Particles synthesized using a temperature of $100^{\circ} \mathrm{C}$ had higher geometric diameters and hydrodynamic diameters compared to systems synthesized with $150^{\circ} \mathrm{C}$ (44).

Solid-state analysis of the MP indicate that the systems exhibit water content values low enough to be ideal for dry powder storage and stability (145). Higher water content is associated with destabilization of amorphous formulations, causing recrystallization of the cargo $(145,213,233)$, which can be detrimental to the formulation and overall therapeutic outcomes. Furthermore, high water content can cause hydrolysis of Ac-Dex and particle cohesion, which would result in larger particle size and a shift in particle deposition toward the upper regions of the lungs (44). DSC data indicate that the transition temperatures of the compounds are $50^{\circ} \mathrm{C}$ greater than their storage conditions, which can allow for product stability (145). Comparison of DSC and PLM data confirm minimal to no crystallinity in the Ac-Dex formulations compared to high crystallinity in mannitol formulations.

The drug release patterns of Ac-Dex MP at acidic and physiological $\mathrm{pH}$ values are similar to previous studies $(35,45,49)$. By controlling the reaction time of AcDex, alterations in the acetalation of dextran altered the release kinetics. However, regardless of Ac-Dex synthesis time, hydrolysis of acetal groups accelerated in acidic conditions, resulting in fast drug release from the NP. The Corrigan model was developed to evaluate initial burst release that occurs in drug delivery systems (234). When exposed to acidic $\mathrm{pH}$ conditions, Ac-Dex degrades quickly, which allows for burst release, resulting in a release pattern similar to those described in a previous report which studied 152 drug release experiments to evaluate burst release (227, 234, 
235). The variation in $\mathrm{K}_{\mathrm{C}}$ values is likely related to differences in the diffusion coefficient and solubility of the drug, as well as the differences in surface area of the systems (235). Since all of the systems encapsulated the same compound and were similar in diameter, the effect of surface area is the likely caused by the different morphologies of the particles (145).

The Peppas-Sahlin kinetics model (236) is based on a two-kinetic constant theory, with $\mathrm{k}_{1}$ relating to the Fickian contribution (diffusion) and $\mathrm{k}_{2}$ relating to the case II relaxation contribution (erosion). Using $\mathrm{k}_{1}$ and $\mathrm{k}_{2}$, the $\mathrm{R} / \mathrm{F}$ ratio was calculated to determine the dominating contribution occurring during drug release (Table SII) (237). For R/F > 1, the case II relaxational contribution dominates, indicating erosion of the delivery system, whereas diffusion is the dominating contribution for $\mathrm{R} / \mathrm{F}<1$. Based on constants $\mathrm{k}_{1}$ and $\mathrm{k}_{2}$, and $\mathrm{R} / \mathrm{F}$ values, drug release from all particle systems at $\mathrm{pH} 5.35$ are predicted to be based on diffusion $\left(\mathrm{k}_{1}<\mathrm{k}_{2}\right.$ and $\mathrm{R} / \mathrm{F}<1$, in all cases) (236, 237). This is likely due to the highly water soluble nature of SRB $(2,236)$.

As stated previously, at pH 5.35 all particle systems exhibited Peppas-Sahlin or Corrigan type kinetics. Peppas-Sahlin showed that release kinetics were based on the diffusion of SRB from the systems rather than erosion of the systems. Corrigan showed that all systems exhibited burst release kinetics, in which all of the encapsulated SRB was released and the kinetics was based on the surface area of the system. Overall, the systems exposed to $\mathrm{pH} 5.35$ exhibited a combination of burst release and exceptionally quick diffusion, likely due to not only the acidic sensitivity of Ac-Dex, but also the high solubility of SRB. At pH 7.4, Fast-Ac-Dex particles exhibited Korsmeyer-Peppas release kinetics, whereas Slow-Ac-Dex exhibited first- 
order release kinetics. Fast-Ac-Dex systems experienced diffusion-based release, likely due to the high solubility of SRB and the extremely slow of erosion of Ac-Dex. Slow-Ac-Dex systems demonstrated short burst release, followed by a highlycontrolled rate of release for SRB, likely due to the high stability (more so than FastAc-Dex) of Slow-Ac-Dex at neutral pH values. Overall, the release kinetics for all systems are ideal for various diseases of the lung, where physiological diseases can be treated with sustained release formulations and diseases associated with acidic conditions (cancer, infectious diseases) can utilize burst release in a controllable manner.

The formulated MP were evaluated using several cell culture techniques as described in the Results. The presence of steady TEER values following exposure of AIC H441 cells to the MP indicates that the systems do not impact the lung epithelium integrity. H441 cells were used for TEER due to their epithelial cell typing. Furthermore, the MP were not toxic to A549, a commonly used lung cancer cell line, at the concentrations and times tested. Finally, results demonstrated that Slow-Ac-Dex MP have the ability to be uptaken into H441, A549, and Calu-3 cells. Uptake is likely due to two phenomena. One explanation is the lipophilicity of the polymer; too high of lipophilicity (Slow-Ac-Dex) can inhibit the uptake of particles in comparison to those that are not as lipophilic. Another explanation could be due to the fact that the cell types are cancerous and inherently have leaky channels. Based on the results, it is likely that both explanations contributed to the uptake of the MP systems. Overall, the results indicate that the MP are likely safe for pulmonary delivery applications.

\subsection{Conclusions}


Ac-Dex MP systems were successfully developed results in the sustained release of a water-soluble compound. Both 'Fast' and 'Slow' MP systems were corrugated in structure, similar in size, and in physical states. The formulations exhibited aerosol dispersion properties that demonstrate they could be used for pulmonary drug delivery. Release of SRB from the formulations was dependent on both the degradation rate of the polymer and the $\mathrm{pH}$ conditions. These factors allowed for burst, sustained, and/or controlled release of the cargo depending on the environment and type of Ac-Dex. In terms of biological interactions, the MP systems showed minimal toxicity and no disruption of epithelial monolayer integrity, while Fast-Ac-Dex particles showed significant uptake compared to their Slow-Ac-Dex counterparts. Overall, the engineered Ac-Dex MP systems developed have the capability of not only delivering water-soluble agents to the whole lung, but also controlling the release of such cargo. These results confirm our previously mentioned hypothesis. 


\title{
CHAPTER 5
}

\author{
COMPARISON OF NANOPARTICLE PURIFICATION PROCESS VIA \\ CENTRIFUGATION AND TANGENTIAL FLOW FILTRATION: A CASE STUDY \\ ON ACETALATED DEXTRAN NANOPARTICLES
}

\begin{abstract}
Submitted for publication in Particuology in April 2019.
Nishan K. Shah ${ }^{1}$, Ryan Ivone ${ }^{1}$, Jie Shen ${ }^{1,2}$, Samantha A. Meenach ${ }^{1,2, *}$

${ }^{1}$ University of Rhode Island, College of Pharmacy, Department of Biomedical and Pharmaceutical Sciences, Kingston, RI 02881

${ }^{2}$ University of Rhode Island, College of Engineering, Department of Chemical

Engineering, Kingston, RI 02881
\end{abstract}




\subsection{Abstract}

Tangential flow filtration (TFF) is a commonly used purification method in a multitude of fields, including particle engineering. Despite this, there is no comprehensive examination on the effects of key parameters of TFF on nanoparticle (NP) outcomes (characteristics). Therefore, this study aims to investigate the influence of various factors on the characteristics of TFF-purified NP, while using centrifugation, the most commonly used NP purification method, as a comparison. In total, this study is divided into three parts: (i) a design of experiment approach to investigate the influence of transmembrane pressure, flow rate, and initial drug loading on NP characteristics using paclitaxel as a model small molecule; (ii) determination of the optimized parameters following statistical analysis; and (iii) evaluation in the variability of NP characteristics following encapsulation of different small molecules.

Once the optimized parameters were determined, paclitaxel tacrolimus and resveratrol-loaded NP were synthesized and purified via centrifugation and TFF. Results indicated that both key parameters and encapsulated drug play significant roles in the outcomes of purified NP, and that TFF can serve as a suitable scale-up purification method, demonstrating significantly smaller NP diameters and increased drug loading in comparison to centrifugation.

\subsection{Keywords}

Tangential flow filtration, nanoparticle purification, Box-Behnken design, acetalated dextran; drug delivery 


\section{$5.3{ }^{1}$ Abbreviations}

(Included as footnote)

\subsection{Introduction}

The application of nanotechnology in biomedical and pharmaceutical sciences has drastically impacted the way diseases are diagnosed and treated $(5,238,239)$. Polymeric nanoparticles (NP) are attractive drug delivery systems, owing to their biocompatibility, tunable characteristics, and environmental responsiveness $(1,2,19)$. Commonly used fabrication methods for polymeric NP include single or double emulsification, nanoprecipitation, and salting out (240). Following nanoparticle fabrication, a purification process is often used to remove additives, surfactants, and/or unencapsulated drug.

The most common method used for NP purification is ultracentrifugation, which utilizes centrifugal force to separate final products from undesired material (24). Similarly, diafiltrating centrifugal devices can be used to separate NP from their raw materials by combining centrifugal forces with the use of filters with a known molecular weight cut off (MWCO) that are added to centrifuge tubes $(24,31)$. While these are simple, easy separation processes, the $g$ forces generated during centrifugation can lead to challenges during the purification process. The compaction of NP by these forces can result in caking and densely packed NP pellets that require

\footnotetext{
12-methoxypropene: 2-MOP; Acetalated dextran: Ac-Dex; Acetonitrile; ACN; Box-Behnken design: BBD; Dimethyl sulfoxide: DMSO; drug loading : DL; encapsulation efficiency: EE; nanoparticle: NP; poly(vinyl alcohol): PVA; paclitaxel : PTX; pyridinium p-toluenesulfonate: PPTS; phosphate buffered saline: PBS; resveratrol: RSV; tacrolimus: TAC; triethylamine: TEA; Trifluoroacetic acid: TFA; tangential flow filtration: TFF; transmembrane pressure: TMP; ultra performance liquid chromatography: UPLC; zeta potential: ZP.
} 
laborious methods to ensure effective redispersion, which may lead to inconsistencies in NP characteristics due to batch-to-batch variation $(241,242)$. Additionally, centrifuges have a finite capacity for containers, hindering the overall feasibility of batch manufacturing via centrifugation.

Tangential flow filtration (TFF) is a commonly-used purification method for biomacromolecules (e.g. proteins), food and beverages, and water filtration (23, 243, 244). Contrary to conventional dead-end filtration methods, filtration by TFF occurs when a fluid containing solutes flows tangentially to a filter, allowing for purification in a dynamic manner. Solutes of interest can be retained, while waste products can be removed. Additionally, a constant concentration gradient is maintained, allowing for the removal of excess media and solute re-concentration (23)

The first study that used TFF processing for NP purification was published in 1993, where polymeric NP were purified following salting out fabrication (245). Since then, numerous studies have utilized TFF systems in NP fabrication processes such as purification and coating $(31,34,246,247)$. In fact, TFF has received significant attention as a consistent and continuous method of purification for liposomal formulations (248).

Despite this work, there has not been a comprehensive examination of the effects of key parameters of TFF on the outcomes of NP characteristics. Although studies and reviews have suggested the impacts that TFF processing parameters (transmembrane pressure (TMP), flow rate, flux, etc.) have on the characteristics of NP formulations (size, charge, dispersity, etc.), there has not been an experimental study to assess these suggestions $(23,24,241,242)$. The purpose of this study was to investigate the effects 
of TFF flow rate and TMP as well as initial drug loading on NP characteristics utilizing a design of experiment (DoE) approach. Acetalated dextran (Ac-Dex) was chosen as the model polymeric carrier, as it has attracted increasing attention as a drug delivery polymer due to its biocompatibility and biodegradability (19). Compared to poly(lactic-co-glycolic acid), Ac-Dex has been shown to be easily tunable and $\mathrm{pH}$ responsive, allowing it to be used for a variety of disease states (44). Moreover, AcDex can be easily produced through a facile one-step reaction, allowing for cost- and time-effective synthesis. Paclitaxel (PTX) was chosen as the model drug in the DoE studies. In addition to PTX, two other hydrophobic small molecules i.e. tacrolimus (TAC) and resveratrol (RSV) were utilized to understand the effect of purification process (centrifugation vs TFF) on the NP characteristics.

\subsection{Experimental Methods}

\subsubsection{Materials}

Dextran from Leuconostoc mesenteroides (9,000-11,000 MW), pyridinium ptoluenesulfonate (PPTS, $\geq 99.9 \%$ ), 2-methoxypropene (2-MOP, $\geq 97 \%$ ), triethylamine (TEA, $\geq 99 \%$ ), anhydrous dimethyl sulfoxide (DMSO, $\geq 99.9 \%$ ), dichloromethane $(\mathrm{DCM}, \geq 99.9 \%)$ and trifluoroacetic acid (TFA) were purchased from Sigma Aldrich (St. Louis, MO, USA). Poly(vinyl alcohol) (PVA), iodine, boric acid $(\geq 99.5 \%)$, potassium iodide ( $\geq 99 \%$ ), acetonitrile (HPLC grade), phosphoric acid (85\%), and phosphate buffered saline (PBS) were purchased from Fisher Scientific (Waltham, MA, USA). Tacrolimus (TAC) and paclitaxel (PTX) were purchased from LC Laboratories (Woburn, MA). Resveratrol (RSV) was purchased from AdipoGen (San 
Diego, CA). The physical characteristics of paclitaxel, tacrolimus, and resveratrol can be found in Supplemental Information.

\subsubsection{Design of experiment study}

\subsubsection{Nanoparticle synthesis and purification via centrifugation}

Acetalated dextran (Ac-Dex) was synthesized using a previously reported method (44) and drug-loaded Ac-Dex NP were synthesized via a single emulsion method. Briefly, PTX and Ac-Dex (40 mg total; 2, 6, or $10 \mathrm{wt} \%$ initial PTX loading) were dissolved in $1 \mathrm{~mL}$ DCM to form the organic phase. $6 \mathrm{~mL}$ of $3 \%(w / v)$ poly(vinyl alcohol) (PVA) in phosphate buffer saline (PBS) (aqueous phase) was added to the organic phase. The organic-water mixture was sonicated (Q500 Sonicator; Qsonica, Newtown, CT) at $70 \%$ amplitude for $30 \mathrm{~s}$ with $1 \mathrm{~s}$ on/off pulses. The resulting emulsion was added to $40 \mathrm{~mL}$ of $0.3 \%$ (w/v) PVA spinning solution and stirred for 4 hours to allow for particle hardening and solvent evaporation. NP formulations were made in triplicate.

\subsubsection{Nanoparticle purification via tangential flow filtration (TFF)}

NP suspensions were made as described above and prior to purification, several batches of NP were combined and added to a TFF system (KRi2 KrosFlo, Spectrum Inc., Rancho Dominguez, CA). The NP suspensions underwent diafiltration using a $500 \mathrm{kDa}$ polyethersulfone-modified (mPES) MidiKros ${ }^{\circledR}$ hollow fiber filter until $15 \mathrm{~mL}$ of concentrated NP remained. $10 \mathrm{~mL}$ of $0.05 \%(\mathrm{w} / \mathrm{v})$ PVA was added to the suspension, which was further concentrated to a final volume of $5 \mathrm{~mL}$. The TFF 
parameters used are outlined in Table 5.1. The resulting NP were frozen at $-20^{\circ} \mathrm{C}$, lyophilized, and stored at $-20^{\circ} \mathrm{C}$.

\subsubsection{Design of experiment using Box-Behnken design}

Box-Behnken design (BBD) was used to examine the impact of the key tangential flow filtration (TFF) parameters on the characteristics of nanoparticle (NP) formulations via a Design Expert software (Version 11, Stat-Ease, Inc. Minneapolis, MN). The factors evaluated were transmembrane pressure (TMP), TFF flow rate, and initial PTX loading in the initial NP formulations. The BBD responses were NP diameter, polydispersity index (PDI), zeta potential, PVA content, and actual PTX loading. To normalize drug content values, PVA content was subtracted from drug loading. The results were analyzed using ANOVA. The complete design and formulation parameter variables are shown in Table 5.1.

Table 5.1 (Top) Tangential flow filtration factors (transmembrane pressure (TMP), flow rate, and initial paclitaxel (PTX) loading) and levels (low, medium, high) for the Box-Behnken Design of nanoparticle formulations. (Bottom) Randomized processing parameters from Design-Expert used to synthesize the initial batch formulations.

\begin{tabular}{|c|c|c|c|}
\hline Factors & Low & Medium & High \\
\hline TMP (psi) & 2 & 11 & 20 \\
\hline Flow Rate (mL/min) & 50 & 100 & 150 \\
\hline Initial PTX Loading (wt \%) & 2 & 6 & 10 \\
\hline Formulation & $\begin{array}{l}\text { TMP } \\
\text { (psi) }\end{array}$ & $\begin{array}{c}\text { TFF Flow Rate } \\
(\mathrm{mL} / \mathrm{min})\end{array}$ & $\begin{array}{c}\text { PTX Loading } \\
\text { (weight \%) }\end{array}$ \\
\hline 1 & 11 & 50 & 10 \\
\hline 2 & 2 & 50 & 6 \\
\hline
\end{tabular}




\begin{tabular}{|c|c|c|c|}
\hline 3 & 11 & 100 & 6 \\
\hline 4 & 20 & 100 & 2 \\
\hline 5 & 11 & 100 & 6 \\
\hline 6 & 20 & 100 & 10 \\
\hline 7 & 2 & 150 & 6 \\
\hline 8 & 20 & 50 & 6 \\
\hline 9 & 2 & 100 & 10 \\
\hline 10 & 11 & 150 & 2 \\
\hline 11 & 2 & 100 & 2 \\
\hline 12 & 11 & 100 & 6 \\
\hline 13 & 11 & 100 & 6 \\
\hline 14 & 11 & 150 & 10 \\
\hline 15 & 11 & 100 & 6 \\
\hline 16 & 11 & 50 & 2 \\
\hline & 20 & 150 & 6 \\
\hline
\end{tabular}

\subsubsection{Nanoparticle characterization}

The hydrodynamic diameters and surface charges of NP were evaluated via dynamic light scattering (DLS) and zeta potential analysis, respectively, using a Malvern Nano Zetasizer (Malvern Instruments, Worcestershire, UK) as previously described (45). In order to determine the actual drug loading in the NP, formulations were dissolved in mobile phase and their drug content was evaluated via ultra performance liquid chromatography (UPLC, LaChrom, Hitachi, Japan), as described in Supplemental Materials. Theoretical and actual drug loading (DL) was calculated using the following equations:

$$
\text { Theoretical DL }=\frac{\text { Initial mass of drug }}{\text { Initial mass of drug + Initial mass of polymer }}
$$

$$
\text { Actual DL }=\frac{\text { Actual mass of drug in NP }}{\text { Mass of NP }}
$$


The amount of PVA in the NP formulations was determined using a previously reported method (Procházková et al., 2014). The actual DL of the NP formulations without PVA was calculated using the following equation:

$$
\text { Actual DL (Less PVA) }=\frac{\text { Mass of drug in NP }}{\text { Mass of NP - Mass of PVA }}
$$

The morphology of the NP formulations was assessed using a Zeiss Sigma VP Field Emission-Scanning Electron Microscope (FE-SEM) (Germany). To demonstrate the presence of the PVA coating, lyophilized NP were suspended in basic water ( $\mathrm{pH} 9)$ and one to two drops of this suspension were transferred to aluminum SEM stubs (TedPella Inc., Redding, CA) prior to air drying. To show particle morphology and size in the absence of the PVA coating, NP were washed once with DI water, and centrifuged at 23,102 $g$ for 2 minutes to remove PVA prior to sample preparation and imaging.

\subsubsection{Comparison of the purification processes}

PTX, tacrolimus (TAC), and resveratrol (RSV)-loaded Ac-Dex NP with 10 wt\% initial drug loading were prepared and purified via either centrifugation or TFF. For centrifugation, deionized (DI) water was added to dilute the NP formulations, which were centrifuged at $19,802 \mathrm{~g}$ for $20 \mathrm{~min}$ to concentrate the NP. The NP were then washed with DI water and centrifuged at 19,802 $g$ for 20 min again. The resulting NP were dispersed in $0.1 \%$ (w/v) PVA to prevent particle agglomeration. Purification via 
TFF was performed as described above, where TMP and flow rate were set at 2 psi and $150 \mathrm{~mL} / \mathrm{min}$, respectively. The NP were then frozen at $-20^{\circ} \mathrm{C}$, lyophilized, and stored at $-20^{\circ} \mathrm{C}$ for further analysis.

In order to understand the impact of the purification method on the final drug and PVA present in the NP formulations, samples were collected at different stages of the purification processes (Figure 5.1) and analyzed. During centrifugation, samples were collected after the first DI water wash and initial centrifugation (Supernatant 1) and after the secondary DI water wash and centrifugation (Supernatant 2). During TFF, samples of the filtrate were collected following the initial diafiltration (Step 1) and concentration (Step 2). Immediately following TFF, the membrane was washed with $70 \%$ ethanol to remove any remaining particles and free drug and this solution was collected for drug and PVA content analysis (Membrane Cleaning, Step 3).

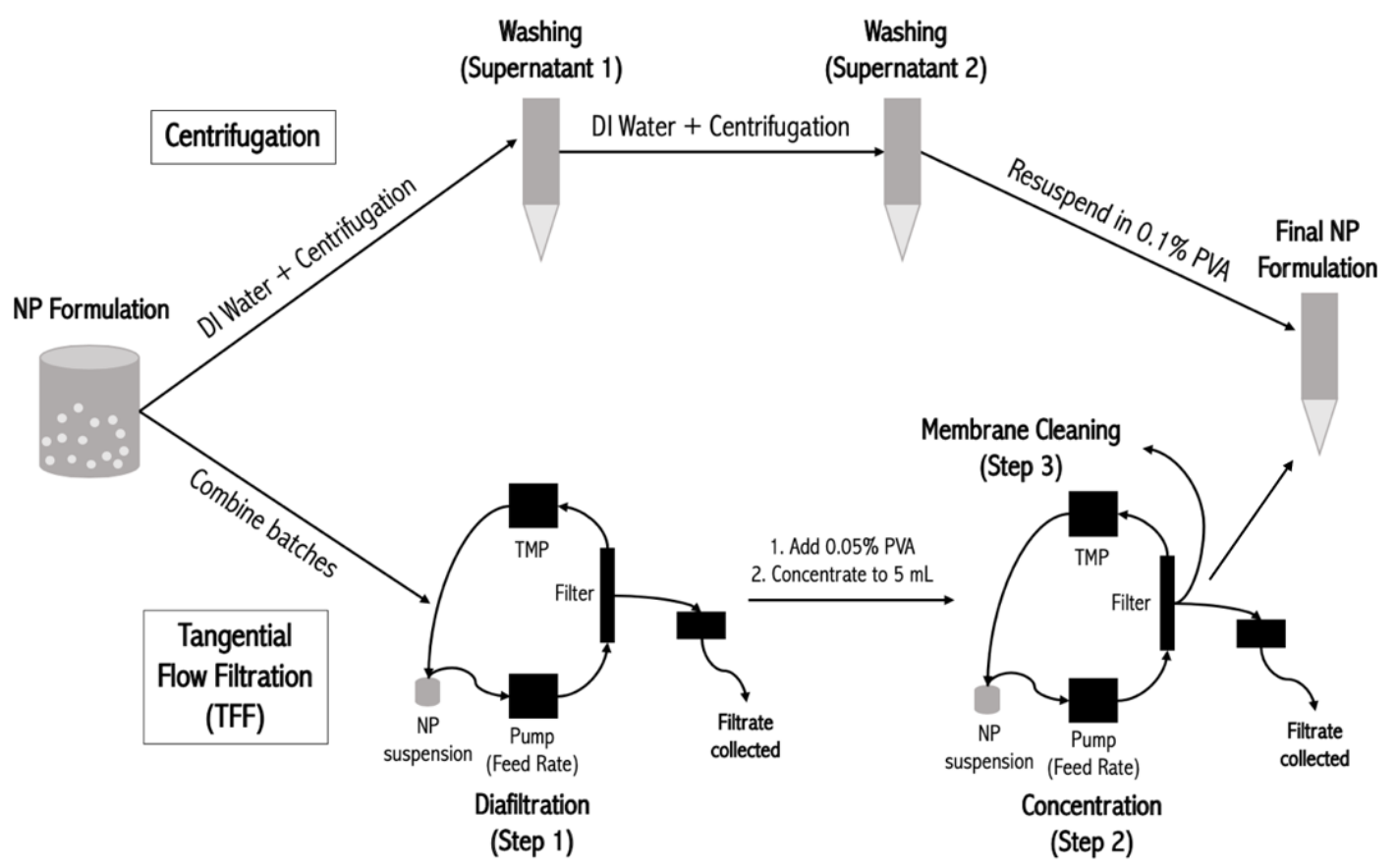

Figure 5.1. Schematic overview of the nanoparticle (NP) purification processes of 
centrifugation and tangential flow filtration (TFF). For centrifugation, deionized (DI) water was added to the NP suspension, following by centrifugation (Washing,

Supernatant 1), this step was repeated (Washing, Supernatant 2), and the NP were resuspended in $0.1 \%$ poly(vinyl alcohol) (PVA). For TFF, the NP suspension underwent an initial filtration (Diafiltration, Step 1), then a concentration step occurred following addition of $0.05 \%$ PVA to get $15 \mathrm{~mL}$ of concentration NP (Concentration, Step 2), and finally the TFF membrane was cleaned to collect residual residue (Membrane Cleaning, Step 3).

\subsubsection{Statistical analysis}

All measurements were performed in at least triplicate and values are given as mean \pm standard deviation. Statistical significance was determined using ANOVA or Student's t-test, where a p-value of $<0.05$ was considered statistically significant. Statistical analysis was performed using either Design Expert software or GraphPad Prism.

\subsection{Results}

\subsubsection{Design of experiment results for PTX-loaded Ac-Dex NP (PTX-NP)}

Figure 5.2 shows the BBD study responses (NP characteristics) from the initial batch formulations including NP diameter, PDI, zeta potential, PVA content, and actual PTX loading. NP diameters following TFF purification ranged from 141 to 172 $\mathrm{nm}$, and the PDI and zeta potential (ZP) values varied slightly from sample-to-sample 
(0.07 to 0.22 and -4.6 to $-1.4 \mathrm{mV}$, respectively). The PVA content in the NP formulations ranged from 0.32 to $0.77 \mathrm{mg}$ PVA/mg NP. Actual PTX loading ranged from 0.026 to $0.086 \mathrm{mg}$ PTX/mg NP whereas PTX loading (less PVA) ranged from 0.052 to $0.23 \mathrm{mg}$ PTX/mg NP.

Table 5.2 and Figure 5.3 show the overall impact of the BBD factors on the experimental responses for PTX-NP purified via TFF. TFF flow rate imparted an inverse effect on the average PDI of TFF-purified NP as determined by the negative coefficient estimate (CE) value and slope of the graph and high $\mathrm{R}$-squared value. Initial PTX loading had a significant impact on NP zeta potential $(\mathrm{p}<0.05)$. On the other hand, initial PTX loading had a positive impact on actual PTX loading as demonstrated by positive $\mathrm{CE}$ and slope as well as high $\mathrm{R}$-squared values. The combination of TMP and flow rate had an impact on the overall diameter of the NP (Figure 5.3, Bottom), where low TMP and high flow rate (or vice versa) allowed for the production of NP with small diameters. The contour maps show how TMP, flow rate, and initial PTX loading had a combined effect on the overall diameter. 

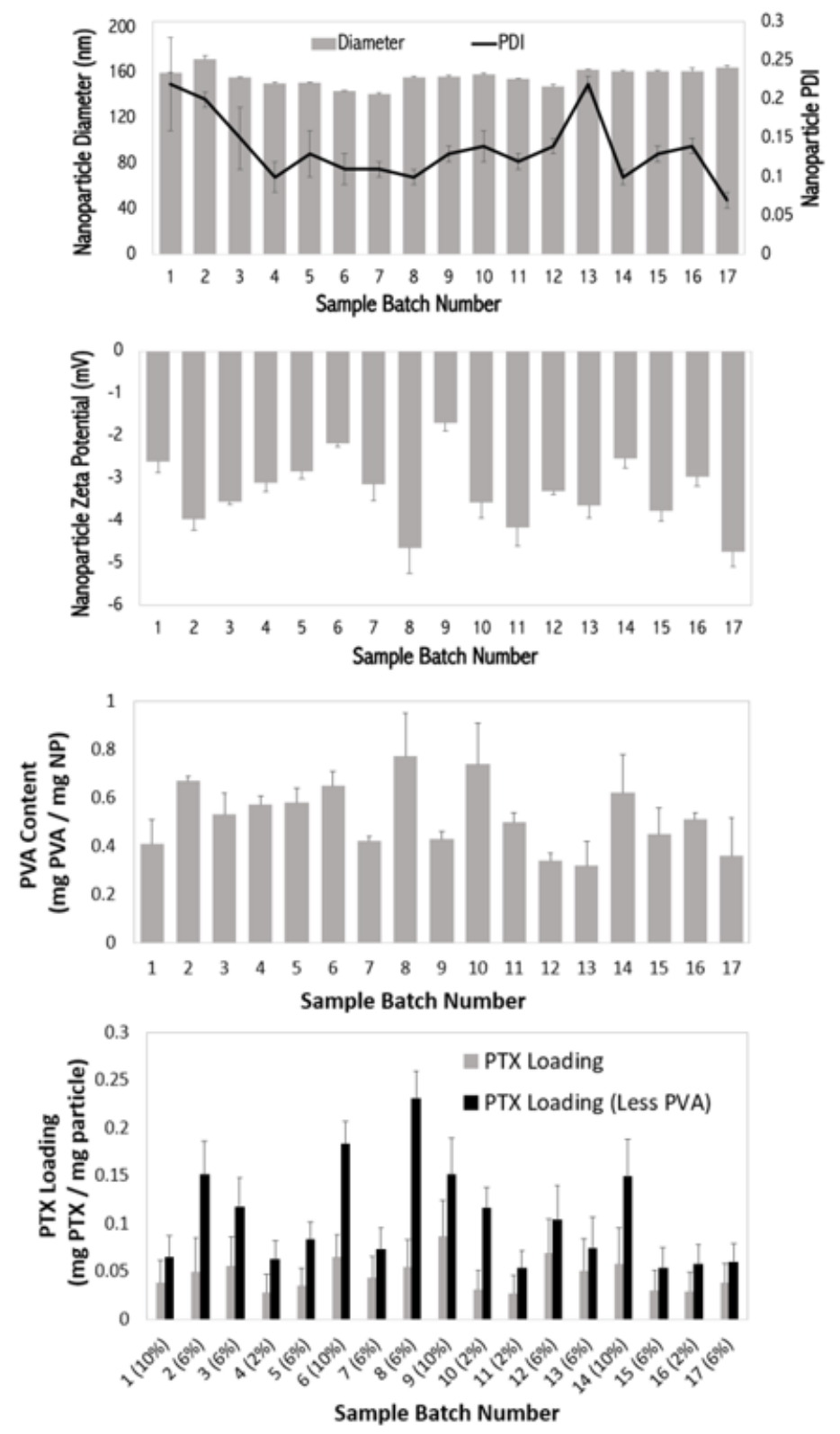

Figure 5.2. Characteristics of paclitaxel-loaded nanoparticles (PTX NP) purified using tangential flow filtration (TFF) including diameter and polydispersity index (PDI); zeta potential; poly(vinyl alcohol) (PVA) content; paclitaxel (PTX) loading (with and without PVA). Mean \pm standard deviation, $\mathrm{n}=3$. 
Table 5.2. Overview of the influences of tangential flow filtration factors (transmembrane pressure (TMP), flow rate, and initial paclitaxel (PTX) loading) on nanoparticle properties, including diameter, polydispersity index (PDI), zeta potential, poly(vinyl alcohol) (PVA) content, actual PTX loading (DL) with and without PVA, and their corresponding coefficient estimates (CE), where applicable. $(---=$ no significance, $*=p<0.05)$.

\begin{tabular}{|c|c|c|c|c|c|c|}
\hline & $\begin{array}{l}\text { Diameter } \\
(\mathbf{n m})\end{array}$ & PDI & $\begin{array}{c}\text { Zeta } \\
\text { Potential } \\
(\mathrm{mV})\end{array}$ & $\begin{array}{c}\text { PVA } \\
\text { Content } \\
\text { (mg } \\
\text { PVA/m } \\
\text { g NP) }\end{array}$ & $\begin{array}{c}\text { Actual } \\
\text { PTX DL } \\
\text { (mg } \\
\text { PTX/mg } \\
\text { NP) }\end{array}$ & $\begin{array}{c}\text { Actu } \\
\text { al } \\
\text { PTX } \\
\text { DL } \\
\text { (Less } \\
\text { PVA } \\
\text { ) (mg } \\
\text { PTX/ } \\
\text { mg } \\
\text { NP) }\end{array}$ \\
\hline $\begin{array}{l}\text { TMP } \\
\text { (psi) }\end{array}$ & --- & --- & --- & --- & --- & --- \\
\hline $\begin{array}{c}\text { Flow } \\
\text { Rate } \\
(\mathbf{m L} / \mathbf{m i} \\
\mathbf{n})\end{array}$ & --- & $\begin{array}{c}* \\
\text { CE: -0.032 } \\
\text { p-value: } \\
0.023\end{array}$ & --- & --- & --- & --- \\
\hline $\begin{array}{c}\text { Initial } \\
\text { PTX } \\
\text { Loading } \\
\text { (wt \%) }\end{array}$ & -- & --- & $\begin{array}{c}* \\
\text { CE: } 0.59 \\
\text { p-value: } \\
0.02\end{array}$ & --- & $\begin{array}{c}* \\
\text { CE: } 0.017 \\
\text { p-value: } \\
0.02\end{array}$ & --- \\
\hline $\begin{array}{l}\text { TMP } \\
\text { and } \\
\text { Flow } \\
\text { Rate } \\
\end{array}$ & $\begin{array}{c}* \\
\text { CE: } 9.96 \\
\text { p-value: } \\
0.014\end{array}$ & --- & --- & --- & --- & --- \\
\hline
\end{tabular}



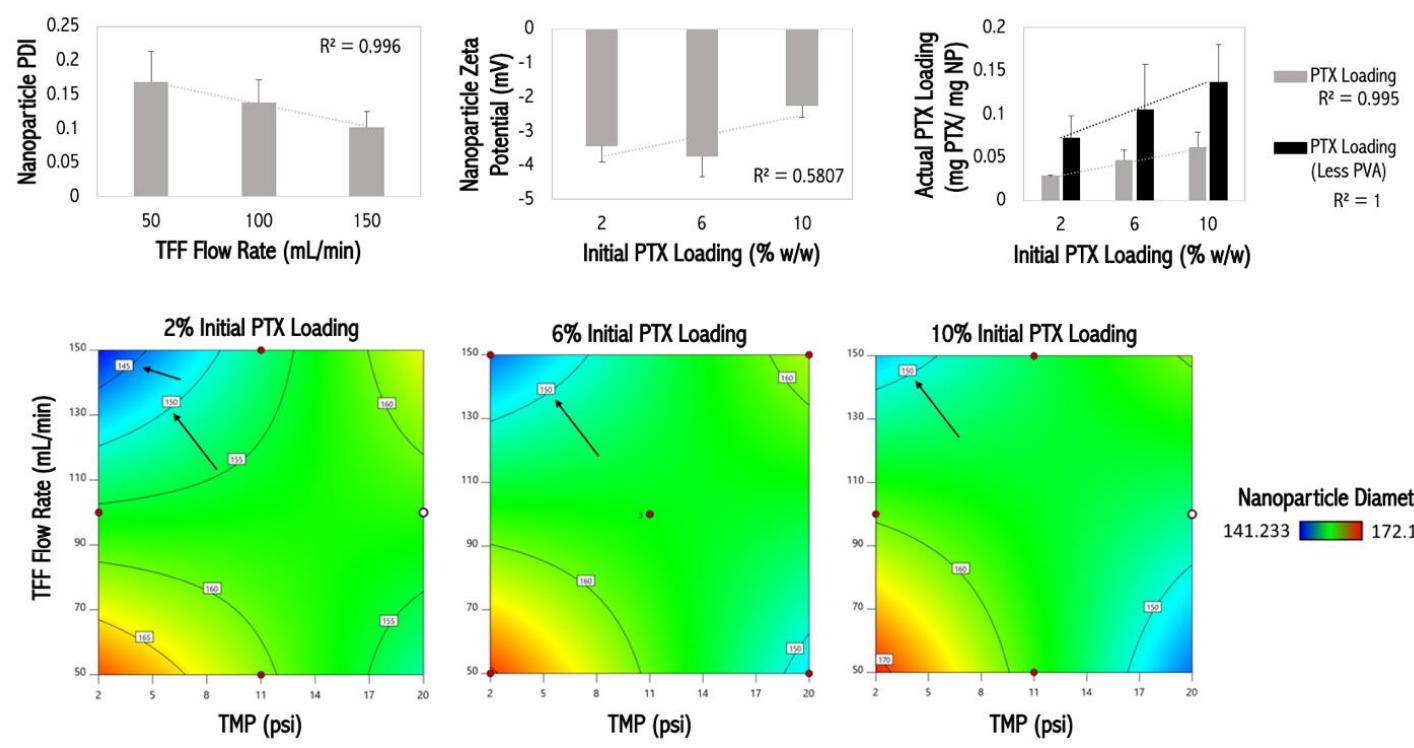

Nanoparticle Diameter $141.233 \square 172.1$

Figure 5.3 Data showing the effect of tangential flow filtration (TFF) process parameters on nanoparticle (NP) characteristics. (Top Left) The effect of TFF flow rate on nanoparticle polydispersion index (PDI). (Top Center) The effect of Initial PTX loading on nanoparticles zeta potential. (Top Right) The effect of initial PTX loading on actual PTX loading (with and without poly(vinyl alcohol), PVA). (Bottom) Contour plots showing the effect of TFF flow rate and transmembrane pressure (TMP) on NP diameter at different initial paclitaxel (PTX) loadings. Regions where nanoparticles exhibited diameters of $150 \mathrm{~nm}$ or less on contour maps are indicated by arrows. 


\subsubsection{Characteristics of PTX-NP, RSV-NP, and TAC-NP}

Following DOE analysis, NP were formulated using optimized parameters using both TFF and centrifugation purification processes to compare their effects and the impact of different drugs on NP characteristics when other small molecules are used. As shown in Figure 5.4, the size of PTX-NP and TAC-NP decreased after TFF and increased after centrifugation, whereas the diameter of RSV-NP decreased after both TFF and centrifugation. In comparison to centrifugation, TFF resulted in significantly smaller-sized particles for PTX-NP and TAC-NP, but not RSV-NP. NP PDI values exhibited no significant change post-purification except for a significant increase for TAC-NP following centrifugation. The zeta potential values of the NP decreased significantly after both purification methods.

PVA content of the NP formulations was significantly higher in TFF-purified NP in comparison to centrifugation-purified NP. Furthermore, PVA content increased for TFF-purified NP (due to the addition of $0.05 \%$ PVA) and decreased for centrifugationpurified NP (due to the washing steps). SEM images of PTX-NP (Figure 5.5) show the morphological similarities of particles purified via TFF compared to particles purified via centrifugation and the extent of PVA coverage in the samples prior to washing. The morphology of NP following TFF and centrifugation purification were spherical with smooth surfaces. The presence of PVA is greater in NP samples purified using TFF in comparison to particles purified via centrifugation, which correlates to the PVA quantification data.

Following TFF, DL values were higher for PTX-NP and RSV-NP, but not for TAC-NP, and DL values (with or without PVA) were higher for PTX-NP and TAC- 
NP, but not for RSV-NP following centrifugation. In addition, DL values were significantly higher for PTX-NP and RSV-NP purified via TFF in comparison to centrifugation, whereas there was no difference for TAC-NP. DL was the highest for PTX-NP and the lowest for RSV-NP following centrifugation. DL increased upon the subtraction of PVA from the samples.
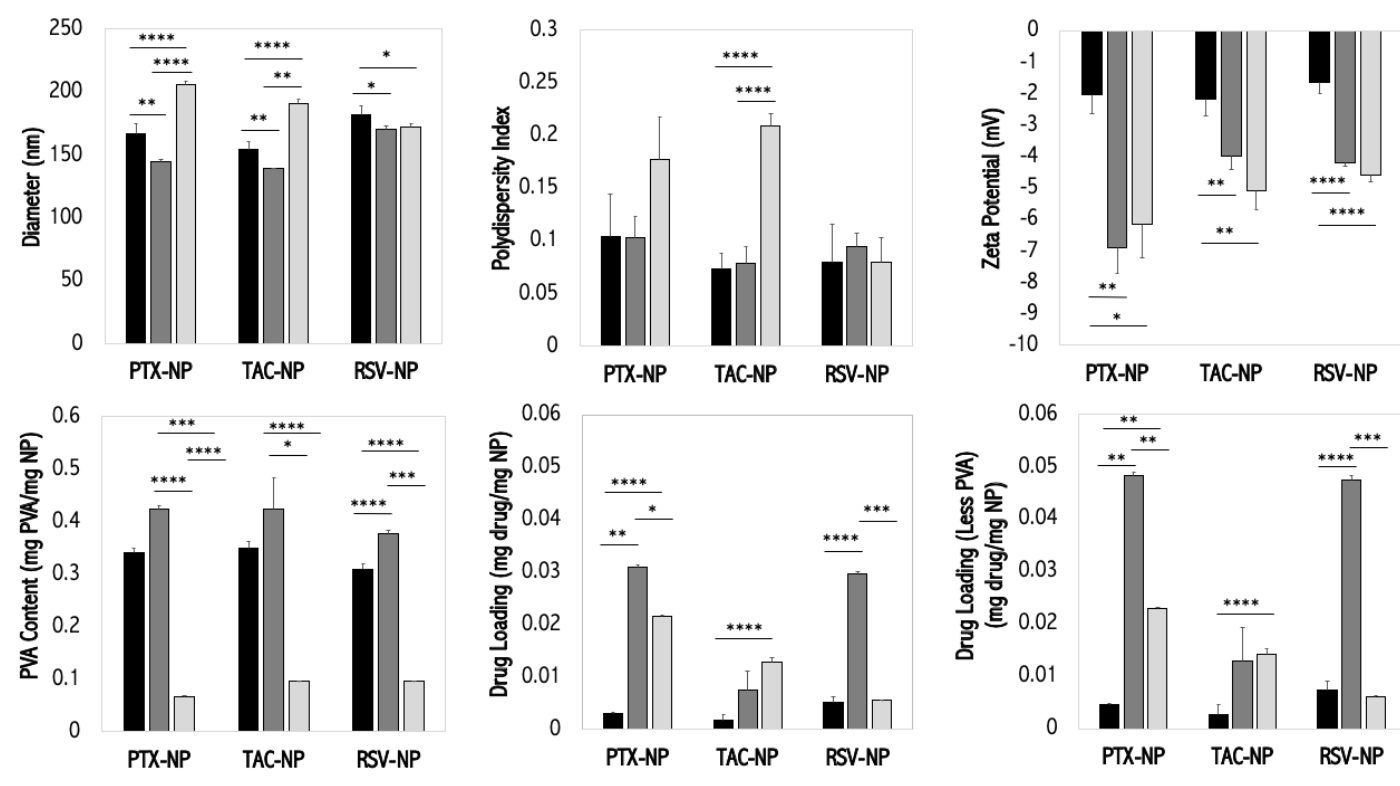

Before Purification $\square$ After TFF $\square$ After Centrifugation

Figure 5.3. Characteristics of paclitaxel (PTX), tacrolimus (TAC), and resveratrol (RSV)-loaded nanoparticles (NP) before purification and after tangential flow filtration (TFF) and centrifugation purification processes. ${ }^{*} \mathrm{p}<0.05, * * \mathrm{p}<0.01, * * * \mathrm{p}$ $<0.001$, and $* * * * \mathrm{p}<0.0001$. Mean \pm standard deviation, $\mathrm{n}=3$. 


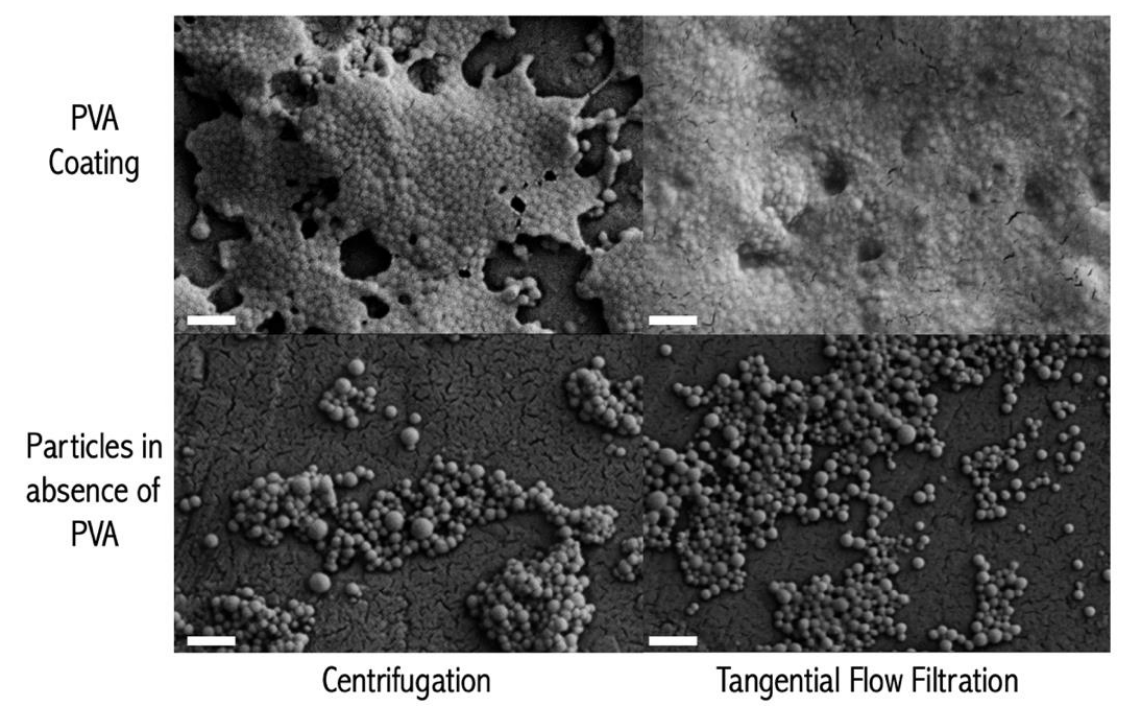

Figure 5.4. Representative scanning electron micrographs of paclitaxel nanoparticle formulations purified via (Left) centrifugation and (Right) tangential flow filtration (TFF). Particles are shown with (Top) poly(vinyl alcohol) (PVA) present and (Bottom) in the absence of PVA. Scale bar $=500 \mathrm{~nm}$.

\subsubsection{Effects of purification steps}

The drug and PVA content of samples taken at different stages of the purification processes (Figure 5.1) were evaluated to understand the effect of the processing steps on these parameters. As shown in Figure 5.6, little drug loss occurred during the concentration step of centrifugation, whereas significant drug loss occurred during the washing step. In correlation, a significant amount of PVA was removed from the NP samples during the centrifugation and less was removed during the washing step. During TFF, there was negligible drug loss during the diafiltration and concentration steps, whereas a significant amount of drug was present for the membrane cleaning step. The PVA content of the samples taken during TFF processing was high for all TFF purification steps. 

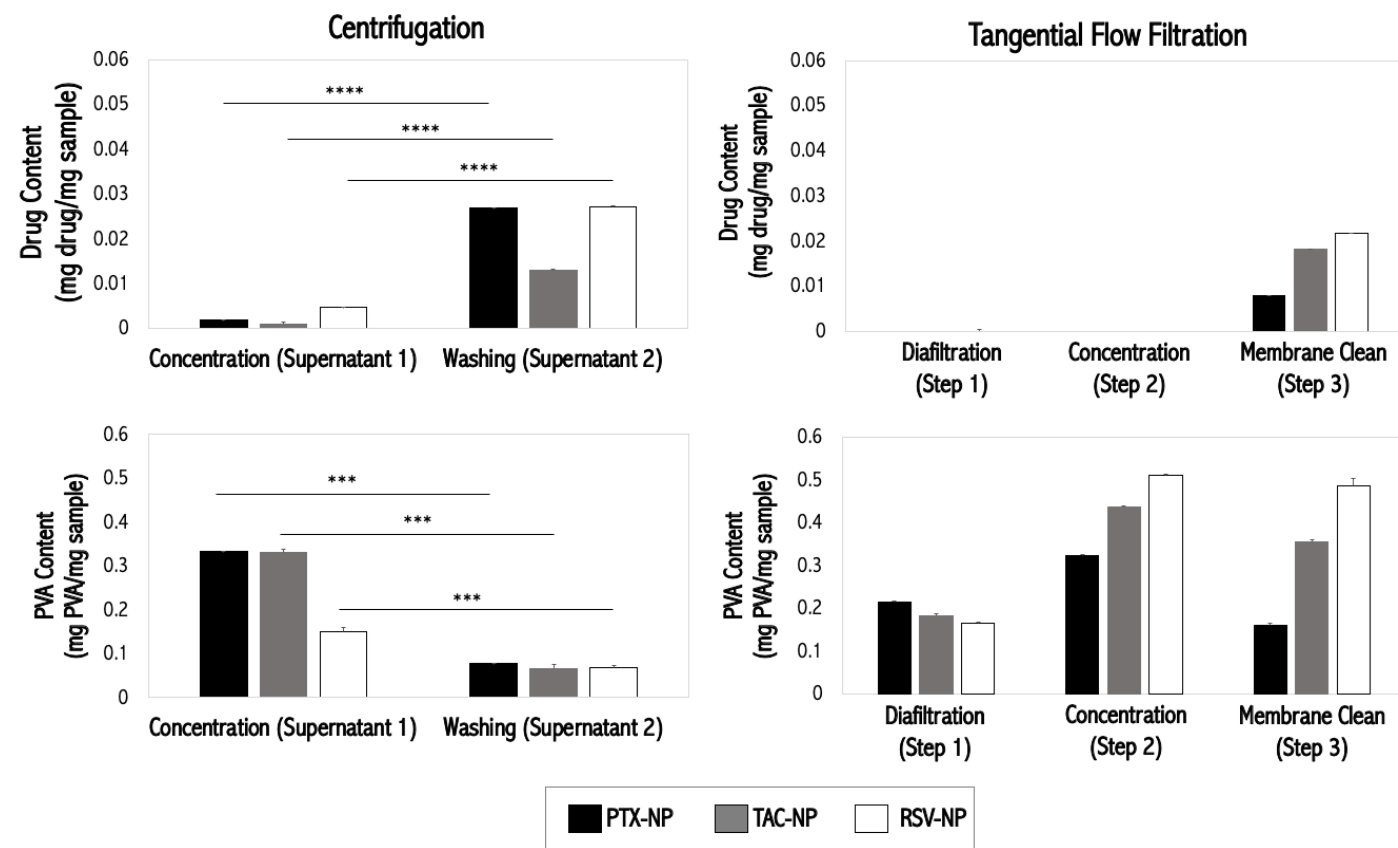

Figure 5.5. Drug loading and poly(vinyl alcohol) (PVA) content of paclitaxel, tacrolimus, and resveratrol-loaded nanoparticle (PTX-NP, TAC-NP, and RSV-NP, respectively) suspensions taken during the centrifugation and tangential flow filtration purification processes. $* \mathrm{p}<0.05, * * \mathrm{p}<0.01, * * * \mathrm{p}<0.001$, and $* * * * \mathrm{p}<0.0001$. Mean \pm standard deviation, $\mathrm{n}=3$.

\subsection{Discussion}

The main goal of this study was to determine the effects of key TFF parameters on NP characteristics. BBD factor levels for TFF purification were chosen based on the manufacturer's recommendations for flow rate and TMP in accordance with the material type and pore size of the membrane used. In addition, centrifugation was used as a comparative purification method. The model molecules used in this study have slightly different chemical properties and structures (taxane, stilbene, and macrolide; 
see Supplement Table S.1); however, they are all lipophilic, which makes them ideal candidates to be encapsulated into polymeric NP. PTX was chosen as the initial model drug due to its extensive use as an FDA-approved chemotherapeutic (249-252). RSV is a well-studied natural product that has demonstrated numerous pharmacological benefits such as anti-inflammatory and anti-cancer properties (253). TAC is one of the most widely used immunosuppressive therapeutics for post-operative organ transplantation (254-256).

Several BBD factors (TFF parameters) had significant influences on the responses (NP characteristics). Varying TFF flow rate had an influence on the PDI of NP, where increasing the flow rate resulted in decreased PDI values. Smaller constituents (e.g. unencapsulated drug or smaller NP) can be more readily filtered out with a higher flow rate, resulting in more uniform particle sizes, and thereby decreasing PDI. This indicates that a high flow rate is desirable to achieve NP with a narrow size distribution. Although zeta potential was significantly influenced by the initial PTX loading (ZP increased as DL increased), the NP can be considered functionally neutral $(79,80)$. The initial PTX loading had a significant influence on the final PTX loading of the NP, where there was a positive correlation.

The combination of flow rate and TMP significantly influenced the size of the NP formulations. By increasing the flow rate, the membrane exposure time decreases, which reduces the potential for membrane fouling. Thus, the resulting concentration gradient at the membrane surface allows smaller permeable species (free polymer, free drug, smaller particles, etc.) to pass through more readily through the membrane via passive diffusion. In turn, an increase in TMP drives more fluid through the 
membrane, thereby increasing filtration capacity, but also increasing the potential of membrane fouling. In addition, increasing the TMP close to or beyond manufacturer recommendations risks the destruction of the membrane itself. Therefore, utilizing a high flow rate and low TMP were determined as the best-case scenario for achieving diameters within the range of 145 to $150 \mathrm{~nm}$. Therefore, based on these results, a lower TMP, higher flow rate, and higher drug loading were selected for further studies.

Following TFF parameter optimization, NP loaded with PTX, RSV, and TAC were formulated using the optimized TFF parameters $(\mathrm{TMP}=2 \mathrm{psi}$, flow rate $=150$ $\mathrm{mL} / \mathrm{min}$, and initial drug loading $=10 \mathrm{wt} \%$ ) and via centrifugation as a comparison of the effect of purification process on NP parameters when different small molecules are used. The increase in diameter and PDI for PTX-NP and TAC-NP following centrifugation was likely due to the agglomeration that occurred during centrifugation process. This phenomenon can potentially be attributed to the challenge in redispersing NP pellets, the fact that smaller NP may not have been removed, and the lower zeta potential values, which may indicated less PVA coating as Ac-Dex is negatively charged. In comparison, the $500 \mathrm{kDa}$ membrane used during TFF was able to retain NP $\geq 50 \mathrm{~nm}$. The differences in diameters observed between TFF-purified NP and centrifugation-purified NP lies in the underlying mechanistic differences between the techniques. Centrifugation utilizes $g$ forces to isolate the NP and is a type of deadend filtration (24). These forces, in addition to redispersion variability, can inherently result in larger NP diameters. In contrast, TFF utilizes a dynamic filtration process, where NP suspensions are constantly flowing tangentially to the membrane, which 
prevents potential agglomeration while allowing for size exclusion purification (241, 242).

The decrease in zeta potential values post-purification can be attributed to the exposure of Ac-Dex on the NP surfaces during processing (Ac-Dex is slightly negatively charged) and overall the NP can be considered functionally neutral (79, 80). During purification via centrifugation, NP suspensions were exposed to PVA-free media and the PVA was washed off the NP. In comparison, NP in the TFF membrane were diafiltrated with PVA-laden media, and as a result, the NP are exposed to more PVA, resulting in higher PVA content. In addition, variations in PVA content of postTFF samples were observed. Since the amount of PVA in suspension varies from batch-to-batch, the PVA content of final formulations often varies (23). NP purification via centrifugation resulted in lower PVA content compared with TFF. Due to its water solubility, PVA diffused into the fresh water during washing and was thereby removed from the surface of the NP during centrifugation. On the other hand, there was a higher volume of PVA-saturated media that remained unchanged in the diafiltration steps during TFF, resulting in higher PVA content for these samples.

It has been shown that centrifugation often results in higher drug loading in comparison to TFF (23). However, in this study, TFF purification resulted in higher drug loading in comparison to centrifugation for PTX-NP and RSV-NP, and resulted in lower drug loading for TAC-NP. Overall, there were no trends related to changes in NP diameter, PDI, zeta potential, PVA content, or drug loading despite the fundamental differences in the physicochemical characteristics (chemical structure, molecular weight, Log P, or water solubility) of the model drugs investigated. 
The large amounts of drug removed from the TFF membrane during membrane washing (Step 3) indicated significant interactions between the free drug or NP and the mPES membrane. The physicochemical characteristics of the model drugs play a role in drug-membrane interactions. For example, the drugs may result in high membrane adsorption due to their high $\log \mathrm{P}$ values. Molecular interactions such as hydrogen bonding (PTX and TAC), non-polar interactions (TAC), and pi-pi stacking (due to the presence of aromatic rings in RSV, PTX, and the mPES membrane) are other possible interactions that likely could have caused drug-membrane interactions, thereby influencing the outcome of the DL values of the formulations.

\subsection{Conclusions}

BBD was used to identify the influence of key TFF parameters, in addition to initial drug loading, on NP characteristics. It was found that NP characteristics were dependent on a multitude of factors, including TFF flow rate and TMP, the initial drug loading, and potential membrane-drug interactions. Upon comparison of the two purification methods, TFF results in difficulties in controlling PVA content and requires consideration for drug-membrane interactions and formulation dependent optimization. In contrast, centrifugation is able to reduce PVA content, but produces shear forces that can affect NP characteristics. TFF circumvents the issues associated with centrifugation. In addition, TFF is an automated process capable of both purification and filtration of NP, resulting in outcomes similar to that of, or better than, centrifugation. 


\title{
CHAPTER 6
}

\section{PHYSICAL CHARACTERIZATION AND FORMULATION DEVELOPMENT OF EACT FOR THE TREATMENT OF PULMONARY ARTERIAL HYPERTENSION}

\author{
Nishan K. Shah ${ }^{1}$, Zimeng Wang ${ }^{2}$, Sweta K. Gupta ${ }^{3}$, Samantha A. Meenach ${ }^{1,3}$ \\ ${ }^{1}$ Department of Biomedical and Pharmaceutical Sciences, College of Pharmacy, \\ University of Rhode Island, Kingston, RI 02881 USA \\ ${ }^{2}$ Phosphorex Inc., Hopkinton, MA 01748 \\ ${ }^{3}$ Department of Chemical Engineering, College of Engineering, University of Rhode \\ Island, Kingston, RI 02881 USA
}




\subsection{Abstract}

Pulmonary arterial hypertension (PAH) is a progressive disease associated with structural remodeling of pulmonary vasculature. The excessive proliferation of the endothelial cells results in thicker vascular walls and eventually in increased pulmonary resistance. Currently, treatment for $\mathrm{PAH}$ is limited to improving quality of life, rather than treating the disease itself. Therefore, alternative approaches are being taken to treat and/or slow the progress of the disease. One approach is through activation of TMEM16A, a calcium-activated chloride channel. Studies have shown that TMEM16A represents a promising therapeutic target for PAH. Recently, a study demonstrated TMEM16A's ability to induce apoptosis of pulmonary endothelial cells via p38-MAPK-induced apoptosis using Eact, a known activator of TMEM16A. Complimenting this previous study, the purpose of this study was to develop a nanocomposite microparticle $(\mathrm{nCmP})$ delivery system for pulmonary administration of Eact. Results thus far demonstrate successful formation of $\mathrm{nCmP}$, as well as their favorable deposition in a Next Generation Impactor. Overall, Eact is a polymorphic small molecule that needs to be further researched to properly develop a stable and usable formulation for the treatment of PAH.

\subsection{Keywords}

Acetalated dextran (Ac-Dex); Eact; pulmonary arterial hypertension; pulmonary drug delivery; nanocomposite microparticles 


\subsection{Abbreviations}

2-methoxypropene (2-MOP), acetalated dextran (Ac-Dex), bone morphogenetic protein receptor-2 (BMPR-2), cyclic-to-acyclic ratio (CAC), dichloromethane (DCM), dimethyl sulfoxide (DMSO), deionized (DI) water, differential scanning calorimetry (DSC), dynamic light scattering (DLS), encapsulation efficiency (EE), Karl Fischer Titration (KFT), nanocomposite microparticles (nCmP), nanoparticles (NP), Next Generation Impactor (NGI), phosphate buffered saline (PBS), poly(vinyl alcohol) (PVA), poly(lactic-co-glycolic acid) (PLGA), pyridium p-toluenesulfonate (PPTS), pulmonary arterial hypertension (PAH), polarized light microscopy (PLM), scanning electron microscopy (SEM), triethylamine (TEA), ultra performance liquid chromatography (UPLC), X-ray diffraction (XRD).

\subsection{Introduction}

Pulmonary arterial hypertension (PAH) is a progressive, life-threatening cardiovascular disease that is defined by an increase in mean pulmonary arterial pressure of the right side of the heart (257-259). This increase in pressure can be traced back to remodeling of the arterial walls, which can result from idiopathic causes (in most cases), or both genetic anomalies and environmental insults $(53,257)$. Ultimately, the remodeling and subsequent thickening of the arterial walls manifests from excessive proliferation and dysfunction of endothelial cells and inflammation (260). Currently, treatments for PAH are based on improving overall cardiopulmonary function and patient quality of life. However, these treatment options are generally unable to inhibit the progress of the disease (53). 
Due to the challenges that lie with current treatment options, newer approaches are being taken to attempt to treat PAH, particularly through focus on cellular-based mechanisms (53). One approach in treating PAH is through improving the function of bone morphogenetic protein receptor-2 (BMPR-2) signaling pathway (259). Mutations to BMPR-2 account for approximately $60-80 \%$ of familial cases of PAH $(255,261$, 262). To capitalize on this approach, tacrolimus (TAC) has been explored as a possible therapeutic approach. Aside from its traditional use as an immunosuppressive agent (254), TAC has been shown to increase BMPR-2 signaling. Clinical evidence has shown improvements in patients suffering from PAH following treatment with lowdose TAC, prompting research to further understand the underlying mechanism and potential use of it as a therapeutic option for the disease (263).

Another approach in the treatment of PAH is the activation of anoctamin-1 (TMEM16A), which, like other calcium-activated chloride channels (CaCCs), is vital for physiological functions, and is expressed in epithelial and non-epithelial cells (264-266). In moments of dysfunction, TMEM16A can be associated with diseases including cancer, cystic fibrosis, and hypertension (265, 267-272). Specifically, an increase in expression of TMEM16A can lead to increased proliferation of cancer cells and a decrease in proliferation of smooth muscle tissue (258). Recently, TMEM16A activation by Eact was shown to promote apoptosis of pulmonary endothelial cells by the p38-MAPK signaling pathway (258). This apoptotic promotion can be a potential treatment method, given that the hyperproliferative phenotype of endothelial cells is a hallmark sign of PAH (258). Eact, the small molecule used in this study, was originally discovered using cell-based high-throughput screening (273). Prior to 2017, 
Eact research had been published in a limited amount of studies, primarily as a control or inducing agent $(269,274,275)$.

Pulmonary delivery has received an increasing amount of attention for the treatment of diseases involving the lungs, primarily due to the potential for localized delivery to tissue and molecular sites of interest $(145,148,276)$. In addition to localized delivery, pulmonary administration offers reduced systemic side effects, a rapid onset of action, and avoidance of first-pass metabolism (135, 277). Dry powder aerosols are a highly attractive method of pulmonary administration owing to the aforementioned advantages plus increased product stability (via absence of water) compared to other dosage forms such as nebulizers (145). Typically, dry powder aerosol particles in the range of $<500 \mathrm{~nm}$ are exhaled due to low mass, while particles that are $1-5 \mu \mathrm{m}$ are likely to deposit in lower regions of the pulmonary system; however, particles within this range are susceptible to macrophage uptake (53). To overcome these obstacles, nanocomposite microparticles can be utilized. A nCmP involves nanoparticles (NP) entrapped in a microparticle matrix. $\mathrm{nCmP}$ maintain the 1 - $5 \mu \mathrm{m}$ size window, allowing for deep lung deposition via sedimentation (45), and following their deposition, $\mathrm{nCmP}$ dissociate to $\mathrm{NP}$, allowing for avoidance of macrophage uptake.

This study involves the development and evaluation of a $\mathrm{nCmP}$ formulation encapsulating Eact, using acetalated dextran (Ac-Dex) as the polymeric carrier. In comparison to poly(lactic-co-glycolic acid) (PLGA), Ac-Dex can be produced using a single-step, facile reaction (53). This reaction can be controlled to alter the acetal coverage of the dextran backbone, allowing for alteration in the release kinetics 
associated with its encapsulated cargo. Additionally, degradation of Ac-Dex produces neutral byproducts (acetone, methanol, dextran) and has been shown to be safe in vivo (19).

\subsection{Materials and Methods}

\subsubsection{Materials}

Unless stated otherwise, all materials were purchased from Sigma Aldrich (St. Louis, MO). Eact was purchased from Tocris Bioscience (Bristol, UK).

\subsubsection{Synthesis and Characterization of Acetalated Dextran}

Acetalated dextran (Ac-Dex) was synthesized using previously described methods with minor modifications (40). $1 \mathrm{~g}$ of lyophilized dextran (MW 9,000 11,000) was dissolved in $10 \mathrm{~mL}$ of anhydrous DMSO with $30 \mathrm{mg}$ of ptoluenesulfonate (PPTS) under nitrogen gas. The reaction was carried out for 3 hours by adding $6.5 \mathrm{~mL} 2$-methoxypropene (2-MOP), and the reaction was quenched after 5 minutes using $1 \mathrm{~mL}$ of triethylamine (TEA). The polymer was precipitated in basic water, filtered, lyophilized, and stored at $-20^{\circ} \mathrm{C}$. The cyclic-to-acyclic (CAC) ratio of Ac-Dex and acetal coverage was verified using ${ }^{1} \mathrm{H}$ NMR (Bruker $300 \mathrm{MHz}, \mathrm{NMR}$, MA) as described previously (54).

\subsubsection{Synthesis of Eact-Encapsulated Nanoparticles}

NP encapsulating $10 \% \quad(w / w)$ Eact were synthesized using single emulsion/solvent evaporation method. $36 \mathrm{mg}$ of Ac-Dex and $4 \mathrm{mg}$ of Eact were 
dissolved in $1 \mathrm{~mL}$ of dichloromethane (DCM) containing $0.01 \%$ (w/w) QD. This organic solution was added to $6 \mathrm{~mL}$ of $3 \%$ poly(vinyl alcohol) (PVA), and the mixture was emulsified using a probe sonicator attached to an ultrasonic processer at $120 \mathrm{~W}$ and $20 \mathrm{kHz}$ for 30 seconds, with 1 second on/off pulses and an amplitude of $70 \%$.

\subsection{4 nCmP Synthesis Via Spray Drying}

nCmP were made using previously established methods, using a Buchi B-290 spray dryer (Buchi Labortechnik, AG, Switzerland) (44, 45). Briefly, NP batches were combined, diluted to $10 \mathrm{mg} / \mathrm{mL}$, and sonicated for 10 minutes prior to spray drying. The spray drying conditions were inlet temperature of $70^{\circ} \mathrm{C}, 0.7 \mathrm{~mm}$ nozzle diameter, atomization gas flow rate of $414 \mathrm{~L} / \mathrm{h}$ (nitrogen), aspiration of $28 \mathrm{~m}^{3} / \mathrm{h}$, pump rate of 0.9 $\mathrm{mL} / \mathrm{min}$, and nozzle cleaner rate of 3 (45). Following spray drying, the resulting $\mathrm{nCmP}$ were allowed to dry in the cyclone under nitrogen gas for an additional 15 minutes. The $\mathrm{nCmP}$ were collected in an amber glass vial and stored at $-20{ }^{\circ} \mathrm{C}$ under desiccation until use.

\subsubsection{Nanoparticle Size and Surface Charge Characterization}

The hydrodynamic diameter and surface charge of the NP and re-dispersed NP were evaluated via dynamic light scattering (DLS) and zeta potential analysis, respectively, using a Malvern Nano Zetasizer (Malvern Instruments, Worcestershire, UK). The parent NP and re-dispersed NP were diluted to $0.25 \mathrm{mg} / \mathrm{mL}$ in DI water, and were analyzed at $25^{\circ} \mathrm{C}$ and an angle of $90^{\circ}$. 


\subsubsection{Particle Morphology and Shape Analysis via Scanning Electron Microscopy}

(SEM)

The shape and morphology of $\mathrm{nCmP}, \mathrm{NP}$, and raw Eact were evaluated by SEM using a Zeiss SIGMA VP Field Emission-Scanning Electron Microscope (FESEM) (Germany). NP samples were concentrated to $15 \mathrm{mg} / \mathrm{mL}$ and $1 \mu \mathrm{L}$ of the resulting suspension was added to aluminum SEM stubs prior to drying (Ted Pella, Inc., Redding, CA). nCmP and raw Eact samples were added directly to double-sided adhesive carbon tabs. The samples were sputter coated with a thin film of gold/palladium alloy under argon gas. Images were taken at $5 \mathrm{kV}$ at various magnifications.

\subsubsection{Crystallinity and Phase Transition Analysis}

Thermal phase transitions of the $\mathrm{nCmP}$ and raw materials were analyzed via differential scanning calorimetry (DSC) using a TA Q10 DSC system (TA Instruments, New Castle, DE) connected to an RSC-90 cooling accessory. 3-5 mg of sample were hermetically sealed in aluminum pans and were placed into the DSC furnace. Samples were heated from $0-230^{\circ} \mathrm{C}$ at a rate of $10^{\circ} / \mathrm{min}(45)$. The crystallinity of the $\mathrm{nCmP}$ and raw materials were examined by X-ray diffraction (XRD) using a Rigaku Multiflex X-ray diffractometer (The Woodlands, TX) as described previously with a scan range of $5-60^{\circ}$ in $2 \Theta$ with a width of 0.1 and scan rate of $1 \% / \mathrm{min}$. (45). Images of the $\mathrm{nCmP}$ and raw materials were observed for birefringence via polarized light microscopy (PLM) using an AmScope polarized microscope (Irvine, CA) (225, 
278). Samples were mounted onto microscope slides, smeared with mineral oil for dispersion, and imaged at 10x at room temperature.

\subsubsection{Karl Fischer Coulometric Titration}

The water content of $\mathrm{nCmP}$ was quantified by Karl Fischer (KF) titration using a $737 \mathrm{KF}$ coulometer (Metrohm, Riverview, FL). $5 \mathrm{mg}$ of powder was dissolved in anhydrous methanol. The solution was injected into the reaction cell filled with Hydranal ${ }^{\circledR}$ KF reagent, and the amount of water was analyzed. Pure solvent was injected as a background sample.

\subsubsection{In Vitro Aerosol Performance}

In vitro aerosol performance of the $\mathrm{nCmP}$ was evaluated using a Next Generation Impactor (NGI, MSP Corporation, Shoreview, MN) equipped with a stainless steel induction port attachment and gravimetric insert cups. The system was coupled with a TPK 2000 critical flow controller and HCP5 vacuum pump (Copley Scientific, UK). The air flow rate (Q) was measured and adjusted to $60 \mathrm{~L} / \mathrm{min}$ prior to each experiment to simulate breath flow by healthy human patients. Fiber glass filters (55 mm, Type A/E, Pall Life Sciences, PA) were placed in the insert cups for stages 1 to 7 for the minimization of MP bounce or re-entrapment (279). Filters were weighed before and after experiments to determine $\mathrm{nCmP}$ amount deposited in each stage. 8-12 mg of powder was loaded into hydroxypropyl methylcellulose capsules (HPMC, size 3, Quali- $\mathrm{V}^{\circledR}$, Quali-caps Inc., Whitsett, NC, USA). The capsules were then loaded into

a dry powder inhaler (HandiHaler, Boehringer Ingelheim Pharmaceuticals, CT) and 
the system was attached to a customized rubber mouthpiece connected to the NGI. Experiments were performed in triplicate with a $10 \mathrm{~s}$ delay and $10 \mathrm{~s}$ running time. For $\mathrm{Q}=60 \mathrm{~L} / \mathrm{min}$, effective cutoff diameters of each stage are as follows: stage 1 (8.06 $\mu \mathrm{m})$, stage $2(4.46 \mu \mathrm{m})$, stage $3(2.82 \mu \mathrm{m})$, stage $4(1.66 \mu \mathrm{m})$, stage $5(0.94 \mu \mathrm{m})$ stage $6(0.55 \mu \mathrm{m})$ and stage $7(0.34 \mu \mathrm{m})$. Fine particle fraction $(\mathrm{FPF})$, respirable fraction (RF), and emitted dose (ED) were calculated using the following equations:

$$
\text { Fine Particle Fraction (FPF) }=\frac{\text { Mass of MP on stages } 2 \text { through } 7}{\text { Total MP mass on all stages }} \times 100 \%
$$

Fine Particle Dose (mg) (FPD) = Mass of MP on stages 2 through 7

$$
\begin{aligned}
& \text { Respirable Fraction }(\mathrm{RF})=\frac{\text { Mass of MP on stages } 2 \text { through } 7}{\text { Initial MP mass loaded into capsules }} \times 100 \% \\
& \text { Emitted Dose (mg) (ED) } \\
& =\text { Inital MP mass in capsules }- \text { Final MP mass in capsules } \\
& \text { Emitted Dose }(E D)=\frac{\text { Initial MP mass in capsules }- \text { Final MP mass in capsules }}{\text { Initial MP mass in capsules }} \times 100 \%
\end{aligned}
$$

The experimental mass median aerodynamic diameter $\left(\mathrm{MMAD} \mathrm{D}_{\mathrm{E}}\right)$ and geometric standard deviation (GSD) for $\mathrm{nCmP}$ were determined using Mathematic ${ }^{\circledR}$ program written by Dr. Warren Finlay (224). 


\subsubsection{Encapsulation Efficiency and Drug Loading Analysis}

Encapsulation efficiency and drug loading of Eact encapsulated in NP were determined using Ultra Performance Liquid Chromatography (UPLC, LaChrom, Hitachi, Japan). Detection of Eact was performed using: $\mathrm{C}_{18} 5 \mu \mathrm{m}$ x $150 \mathrm{~mm}$ x $4.6 \mathrm{~mm}$ column (Ascentis, Sigma-Aldrich, St. Louis, MO), $1 \mathrm{~mL} / \mathrm{min}$ pump rate, mobile phase of 70:30 acetonitrile: $0.1 \%$ (30\% phosphoric acid) aqueous solution, absorbance at 280 $\mathrm{nm}$ and temperature of $50^{\circ} \mathrm{C}$. Drug loaded NP were fully dissolved in mobile phase prior to analysis. The EE and DL of Eact loaded NP were determined by the following equations:

$$
\begin{gathered}
\text { Theoretical DL }=\frac{\text { Initial mass of drug }}{\text { Initial mass of drug + Initial mass of polymer }} \\
\text { Actual DL }=\frac{\text { Actual mass of drug in NP }}{\text { Mass of NP }} \\
\mathrm{EE}=\frac{\text { Actual DL }}{\text { Theoretical DL }} \times 100 \%
\end{gathered}
$$

\subsubsection{Statistical Analysis}

All measurements were performed in at least triplicate. Statistical significance was determined using Student's t-test. A p value of $<0.05$ was considered as statistically significant. Values were presented as mean \pm standard deviation unless stated otherwise. 


\subsection{Results and Discussion}

\subsubsection{Morphology Analysis of NP and $n C m P$}

In this study, 3-hour Ac-Dex was used for nanoparticle (NP) synthesis due to its ability to control the release of therapeutic cargo at physiological conditions for a longer period of time than 5-min Ac-Dex $(36,45)$. This phenomenon is due to the higher ratio of cyclic acetal groups to acyclic groups in 3-hour Ac-Dex compared to 5min Ac-Dex, allowing for slower degradation over time (35). In previous studies, it was shown that both 5-min and 3-hour Ac-Dex allowed for sustained release of various lipophilic compounds, however, 3-hour Ac-Dex resulted in longer release of these compounds $(35,43,45)$.

As shown in Table 6.1, Eact-loaded NP were $191 \mathrm{~nm}$ in diameter, with favorable polydispersity (0.11) and surface charge (functionally neutral; $-4.18 \mathrm{mV}$ ). The loading of Eact in the Eact NP formulation was $27.6 \mu \mathrm{g}$ Eact/mg NP with an overall Eact encapsulation efficiency of $25.9 \%$. Following spray drying, nCmP were re-dispersed in DI water to allow for dissociation into parent Eact NP. Like parent NP, re-dispersed Eact-loaded NP were less than $200 \mathrm{~nm}$ in diameter, with similar PDI (0.11) and surface charge $(-4.20 \mathrm{mV})$. Statistical analysis showed that the two groups were not significantly different from one another $(\mathrm{p}>0.05)$. Parent Eact NP and Eact NP re-dispersed from nCmP exhibited smooth and spherical morphology, whereas nCmP were agglomerations of NP with corrugated structure (Figure 6.1). 
Table 6.1. Physical characteristics of Eact-loaded nanoparticles (NP) and nanocomposite microparticles $(\mathrm{nCmP})$ including the diameter, polydispersity index (PDI), surface charge via zeta potential, drug loading, encapsulation efficiency (EE), water content, and PVA surface content.

\begin{tabular}{|c|c|c|c|c|c|}
\hline & $\begin{array}{l}\text { Diameter } \\
(\mathbf{n m})\end{array}$ & PDI & $\begin{array}{c}\text { Charge } \\
(\mathrm{mV})\end{array}$ & $\begin{array}{c}\text { Drug Loading } \\
\text { ( } \mu \text { g Eact } / \mathbf{m g} \\
\text { NP) }\end{array}$ & $\begin{array}{c}\text { EE } \\
\%\end{array}$ \\
\hline Blank NP & $157.6 \pm 2.3$ & $0.10 \pm 0.02$ & N/A & N/A & N/A \\
\hline Eact NP & $190.9 \pm 8.2$ & $0.11 \pm 0.05$ & $-4.18 \pm 2.45$ & $27.6 \pm 8.8$ & $\begin{array}{c}25.9 \\
\pm \\
8.6\end{array}$ \\
\hline $\begin{array}{l}\text { Re-Dispersed } \\
\text { Eact NP }\end{array}$ & $196.8 \pm 2.0$ & $0.11 \pm 0.02$ & $-4.20 \pm 0.92$ & N/A & N/A \\
\hline Eact $n C n$ & \multicolumn{2}{|c|}{ Water Content (\%) } & \multicolumn{2}{|c|}{$\begin{array}{l}\text { PVA Surface Content } \\
(\mathrm{mg} \mathrm{PVA} / \mathrm{mg} \mathrm{nCmP)}\end{array}$} & \\
\hline
\end{tabular}
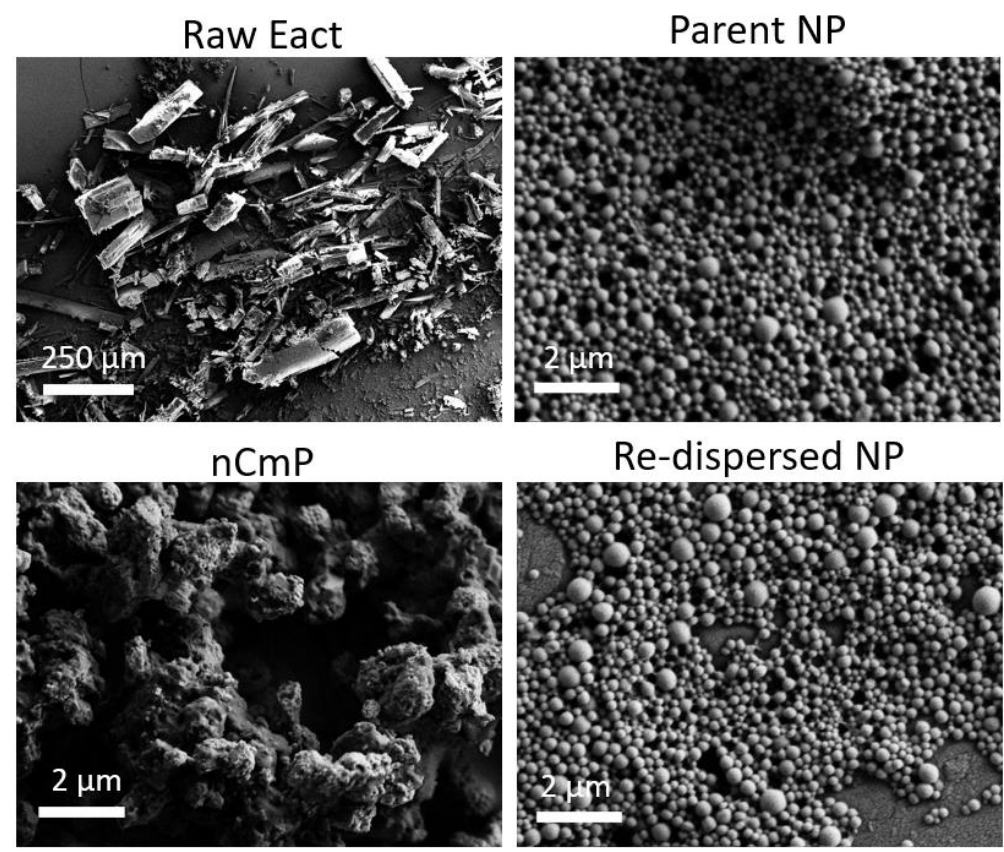

Re-dispersed NP

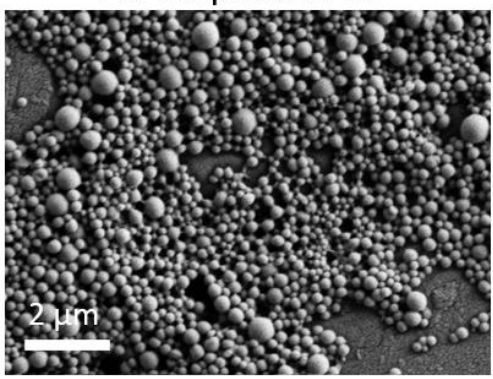

Figure 6.1. SEM micrographs of raw Eact, parent Eact nanoparticles (NP), Eact nanocomposite microparticles (nCmP), and NP re-dispersed from $\mathrm{nCmP}$. 
The formation of the corrugated $\mathrm{nCmP}$ structures are a result of the spraydrying process (45). Following suspension of NP in water, the suspension is exposed to the drying gas, resulting in NP dispersed in droplets. As water evaporates, NP are transported to the outside of the droplet due to droplet saturation (280). Once drying occurs the droplet walls collapse, resulting in corrugation (145). The hollow structure of the particles results in lower particle density, altering the overall aerodynamic diameter, and increasing flowability during patient inspiration (146). Additionally, due to their ability to dissociate into $\mathrm{NP}$ following sedimentation, $\mathrm{nCmP}$ are able to delivery NP to the latter depths of the lungs (44).

\subsubsection{Solid-State Analysis of $n \mathrm{CmP}$}

$\mathrm{X}$-ray diffractograms of raw Eact, Eact $\mathrm{nCmP}$, and raw 3-hour Ac-Dex are shown in Figure 6.2A. Raw Eact exhibited strong signals corresponding to crystallinity, whereas Ac-Dex showed no distinct peaks, indicating an amorphous physical characteristic. Eact $\mathrm{nCmP}$ exhibited fewer peaks, although several strong visible peaks were present. It is possible that the lack of peaks is due to a detection limitation of the equipment, in addition to the lower drug loading. DSC thermograms of raw Eact showed a melting peak at $105^{\circ} \mathrm{C}$ (Figure 6.2B), whereas no observable transitions were observed for raw 3-hour Ac-Dex. Following spray drying and formation of the $\mathrm{nCmP}$, two endothermic peaks were seen at $155^{\circ} \mathrm{C}$ and $200^{\circ} \mathrm{C}$ for Eact $\mathrm{nCmP}$, corresponding to possible polymorphs of Eact. Prior to the melting of Eact $\mathrm{nCmP}$ at $200^{\circ} \mathrm{C}$, brief re-crystallization may have occurred at $155^{\circ} \mathrm{C}$, signifying an increase in molecular mobility and subsequent re-crystallization to form another 
crystalline structure $(281,282)$. Polarized light microscopy (PLM) images (Figure 6.2C) corresponded to both XRD and DSC profiles, signifying amorphous characteristics for raw 3-hour Ac-Dex, crystallinity of raw Eact, and crystallinity of Eact $\mathrm{nCmP}$, as evident by the birefringency in the polarized light samples. Combining these data with the low water content of the $\mathrm{nCmP}(<5 \%)$ (Table 6.1) provides evidence that the formation of $\mathrm{nCmP}$ altered the physical characteristics of Eact to a less crystalline state while maintaining minimal water content (145). Results indicate that Eact is a crystalline small molecule, however, more studies need to be carried out to assess if polymorphs exist and if molecular rearrangements occurred $(281,283$, 284).
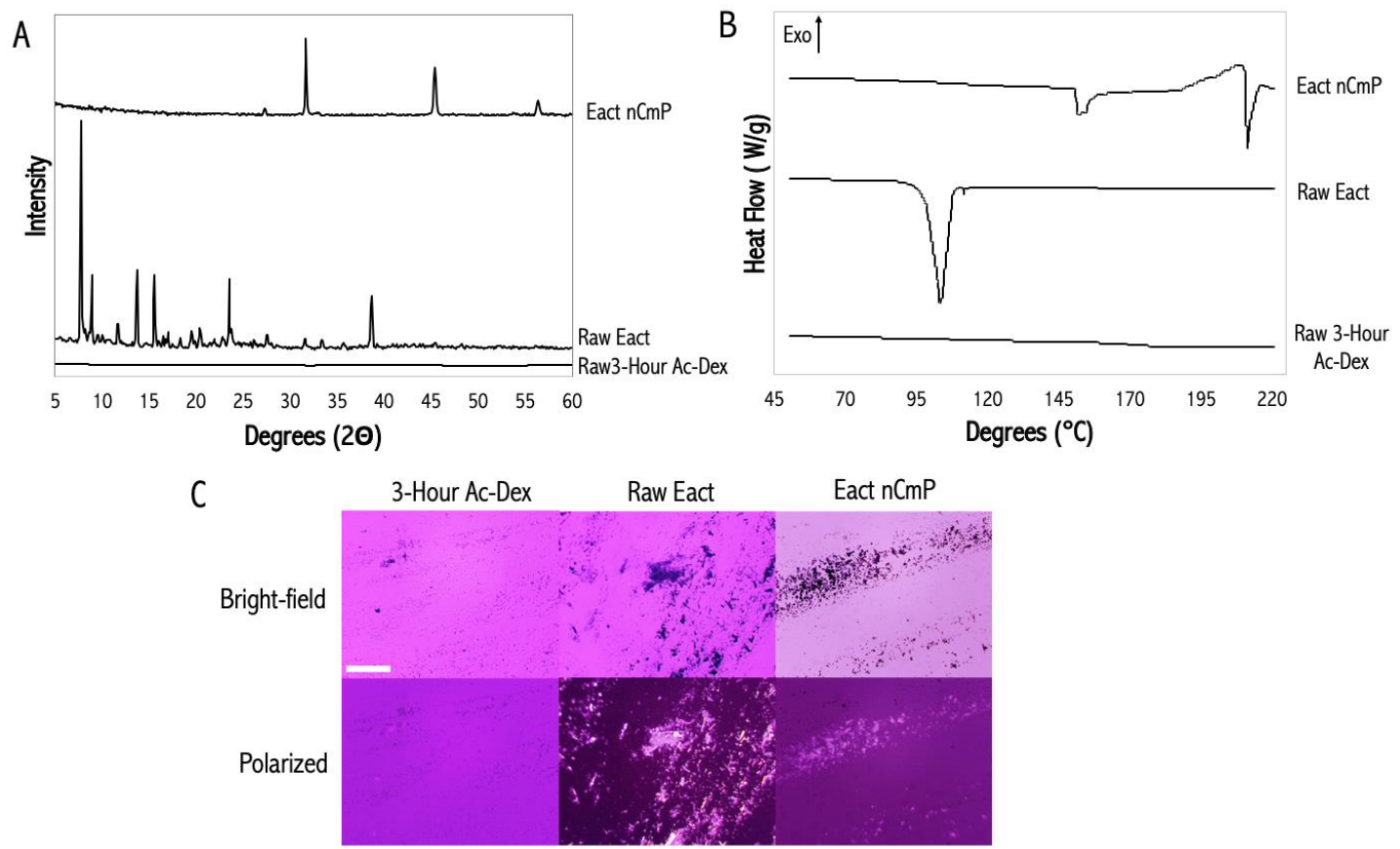

Figure 6.2. (A) Representative x-ray diffractograms, (B) representative differential scanning calorimetry thermograms and (C) polarized light microscopy images of raw materials (3-hour Ac-Dex and Eact) and Eact-loaded nCmP. Scale bar $=0.1 \mathrm{~mm}$. 


\subsubsection{Aerosol Performance Characterization of $n \mathrm{CmP}$}

In vitro aerosol performance of $\mathrm{nCmP}$ was evaluated using a Next Generation Impactor (NGI) coupled to a dry powder inhaler. $\mathrm{nCmP}$ deposited on all stages of the NGI (Figure 6.3), indicating the potential for the particles to deposit in multiple areas

of the lungs. The high fine particle fraction (>96\%, Table 6.2) indicates that a significant portion of the particles are capable of aerosolization $(53,54)$. In addition, RF and ED were similar or higher than previously published studies, and indicate that inhalation of $\mathrm{nCmP}$ aerosols would likely result in effective particle delivery to the lungs. $M M A D_{E}$ values were within the $1-5 \mu \mathrm{m}$ range necessary for deep lung deposition (45), while geometric standard deviation (GSD) was higher compared to previous studies $(45,49,53,153,285)$. The high GSD is likely due to unequal agglomeration of the NP when forming the $\mathrm{nCmP}$ (44). PAH is a cardiopulmonary related disease, and would benefit from deep lung deposition due to the high vascularization of alveolar tissue and location of the arterial walls of the heart (53, 142, 257). While MP have shown the capability for deep lung deposition, a previously published study evaluated the aerosol performance between $\mathrm{nCmP}$ and MP. Results indicated that $\mathrm{nCmP}$ were able to better deposit in latter stages of the NGI compared to MP. Due to the location of $\mathrm{PAH}$, utilization of $\mathrm{nCmP}$ formulations may be more beneficial than MP (45). 


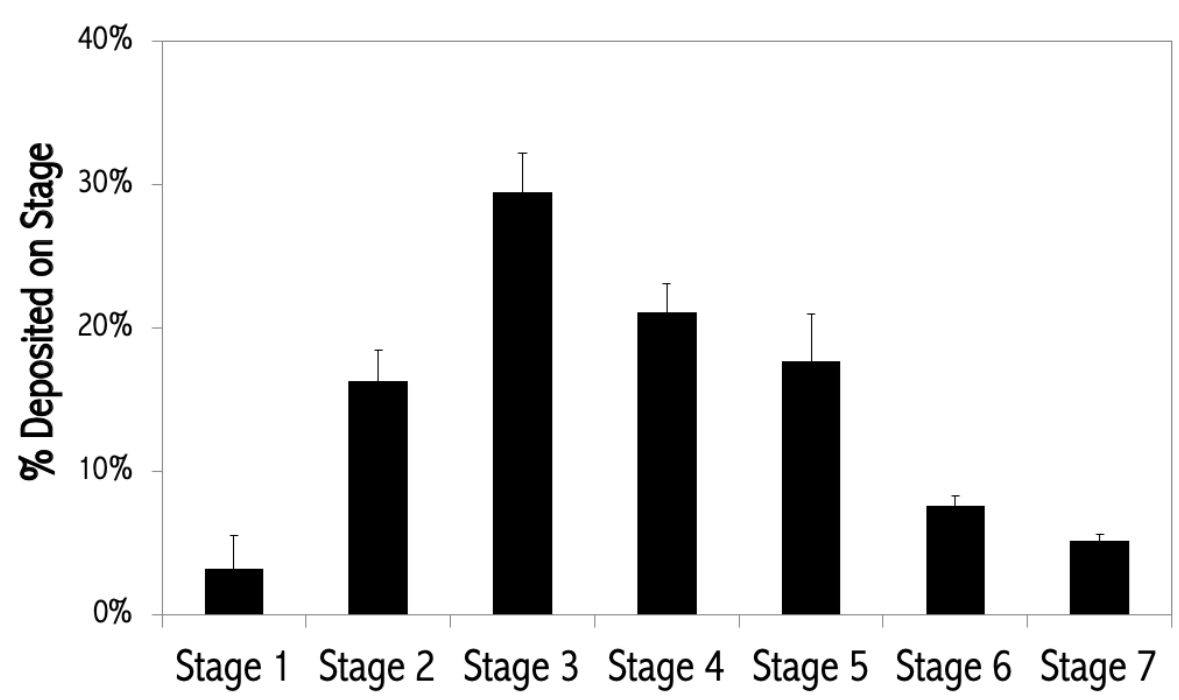

Figure 6.3. Aerosol dispersion analysis of Eact-loaded nanocomposite nanoparticles (Eact $\mathrm{nCmP}$ ) in stages 1-7 of a Next Generation Impactor, expressed as percent particle deposition per stage (mean \pm standard deviation, $\mathrm{n}=3$ ). For $\mathrm{Q}=60 \mathrm{~L} / \mathrm{min}$, cutoffs for each stage of the NGI are: $8.06 \mu \mathrm{m}$ (stage 1), $4.46 \mu \mathrm{m}$ (stage 2), $2.82 \mu \mathrm{m}$ (stage 3), $1.66 \mu \mathrm{m}$ (stage 4), $0.94 \mu \mathrm{m}$ (stage 5), $0.55 \mu \mathrm{m}$ (stage 6) and $0.34 \mu \mathrm{m}$ (stage 7).

Table 6.2. Aerosolization performance properties of spray-dried Eact-loaded nanocomposite microparticles (Eact $\mathrm{nCmP}$ ), including fine particle dose (FPD), fine particle fraction (FPF), respirable fraction (RF), emitted dose (ED), experimental mass median aerodynamic diameter (MMAD $\mathrm{EXP}_{\mathrm{P}}$ ), and geometric standard deviation (GSD) via Next Generation Impactor analysis (mean \pm standard deviation, $\mathrm{n}=3$ ).

\begin{tabular}{|l|c|c|c|c|c|c|c|}
\hline Particles & $\begin{array}{c}\text { FPD } \\
(\mathbf{m g})\end{array}$ & $\begin{array}{c}\text { ED } \\
(\mathbf{m g})\end{array}$ & $\begin{array}{c}\text { FPF } \\
(\boldsymbol{\%})\end{array}$ & $\begin{array}{c}\mathbf{R F} \\
(\boldsymbol{\%})\end{array}$ & $\begin{array}{c}\text { ED } \\
(\boldsymbol{\%})\end{array}$ & $\begin{array}{c}\text { MMADEXP } \\
(\mu \mathbf{m})\end{array}$ & $\begin{array}{c}\text { GSD } \\
(\boldsymbol{\mu m})\end{array}$ \\
\hline Eact & $17.2 \pm$ & $25.3 \pm$ & $96.8 \pm$ & $70.5 \pm$ & $98 \pm$ & & $2.88 \pm$ \\
nCmP & 0.4 & 0.5 & 2.3 & 0.3 & 1.4 & $2.68 \pm 0.24$ & 0.34 \\
\hline
\end{tabular}




\subsection{Conclusions}

An aerosol nCmP system was developed for the delivery of the novel therapeutic Eact that can be used to treat pulmonary arterial hypertension. Results thus far show that Eact was successfully encapsulated in 3-hour Ac-Dex NP and that the resulting $\mathrm{NP}$ were successfully formulated into $\mathrm{nCmP}$. The size of $\mathrm{NP}$ before $\mathrm{nCmP}$ synthesis and after dissociation from the $\mathrm{nCmP}$ were below $200 \mathrm{~nm}$ and were similar with respect to surface charge and PDI, providing evidence that NP characteristics were not altered following encapsulation in and release from $\mathrm{nCmP}$. XRD data indicated changes in Eact crystallinity following $\mathrm{nCmP}$ formation. In addition, $\mathrm{DSC}$ thermograms showed the presence of two crystalline states in Eact $\mathrm{nCmP}$, indicating the polymorphic nature of Eact. The water content of $\mathrm{nCmP}$ were below $5 \%$. Finally, $\mathrm{nCmP}$ showed favorable aerosol performance, capable of depositing in all stages of the NGI. Overall, Eact-encapsulated $\mathrm{nCmP}$ may be a favorable delivery system for Eact for the treatment of PAH in a patient convenient and effective manner. However, the polymorphic nature of Eact must be evaluated further and the crystallinity of Eact may need to be controlled through the use of additional polymers.

\subsection{Acknowledgements}

The authors thank Dr. Gaurav Choudhary M.D and the Vascular Research Laboratory for the purchase of Eact, and $\mathrm{RIN}^{2}$ for SEM and XRD access. Additionally, this work was made possible by the use of equipment and services available through the RI-INBRE Centralized Research Core Facility. 


\section{CHAPTER 7}

\section{CONCLUSIONS AND FUTURE WORK}

\subsection{Conclusions}

This primary focus of this dissertation was on the exploration of the polymer acetalated dextran (Ac-Dex) as a versatile drug carrier that has the potential to overcome contemporary obstacles in drug delivery. One of the main obstacles faced in drug delivery applications is drug toxicity and a cause of this is the typical lack of active targeting for drug delivery systems to reach desired tissues. To actively target macrophages in an effort to combat this obstacle, Ac-Dex nanoparticles (NP) were coated in phosphatidylserine (PS) to mimic apoptotic cells. Results indicated minimal toxicity of the NP delivery systems, as well as significant PS NP uptake by macrophages, providing evidence that active targeting is achievable using Ac-Dexbased nanoparticle formulations.

Sustained release of therapeutics is an ongoing research interest, for both lipophilic and hydrophilic entities. However, the release of hydrophilic entities is inherently more difficult to control, given their solubility in biological medium and the inability to control the release of these entities can generate another avenue for drug toxicity. Taking advantage of Ac-Dex's hydrophobicity and tunability, multiple aerosol powder formulations were synthesized via spray drying to modify the release behavior of a highly water-soluble dye. Results show that the release kinetics were both $\mathrm{pH}$ and 
polymer tunability dependent, where sustained release was reached in physiological conditions and controlled release was achieved in acidic conditions. In addition, 3hour and 5-minute Ac-Dex experienced differences in their kinetic analysis, where the former resulted in slower release of a water-soluble compound.

During the production of nanoscale delivery systems, purification methods are employed to ensure that the desired product is collected and packaged prior to usage. However, conventional methods of purification may be inefficient, especially when scale-up approaches are needed. To overcome this challenge, tangential flow filtration was evaluated using a DoE approach to understand the influence of key parameters on NP characteristics using Ac-Dex as a model carrier. Following this, a set of parameters were chosen, and using these parameters, NP were synthesized, encapsulating one of three small molecules. Results show that tangential flow filtration is a suitable purification method but requires drug, system, and external factor considerations.

$\mathrm{PAH}$ is a life-threatening disease with no known treatment options. Current therapeutics for the disease rely on improving quality of life rather than slowing the progress of the disease. Eact, a TMEM16A activator, has been shown to induce apoptosis of hyperproliferating pulmonary endothelial cells, and can serve as a possible route of treatment. Utilizing the rationale described previously, $\mathrm{nCmP}$ were developed with the intention of pulmonary administration to increase localization and subsequent absorption of Eact into the cardiopulmonary regions of the body. Preliminary data shows that $\mathrm{nCmP}$ encapsulating Eact were successful in depositing in latter stages of an NGI, simulating deep lung deposition. It was also determined that 
Eact is a highly-crystalline, polymorphic small molecule that requires further exploration into both the polymorphs and possible formulation development.

\subsection{Future Work}

Although this dissertation provides a comprehensive evaluation of Ac-Dex and its promise as a drug carrier, there are still opportunities to further research the biopolymer. These opportunities include

1. The use of Ac-Dex in other delivery systems not mentioned here or in literature.

2. Investigation of Ac-Dex-based systems designed here using disease models and active pharmaceuticals.

3. Use of in vivo models to demonstrate the potential of the systems described and researched in this dissertation.

4. Completion of studies that were based on preliminary data.

5. Usage of other polymers to aid in Ac-Dex's encapsulation. 
APPENDIX A

Supplementary Material for Chapter 3 
(A)

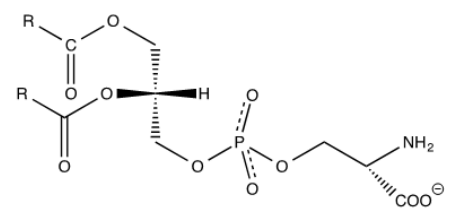

(B)

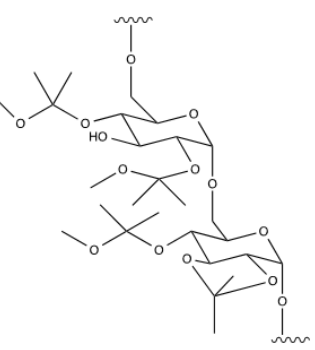

(C)

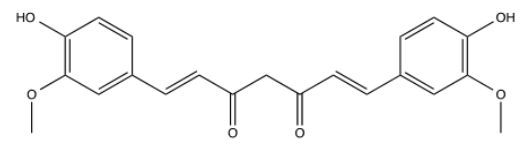

Figure A1. Chemical Structures of DPPS (A), Ac-Dex (B), and Curcumin (C). 


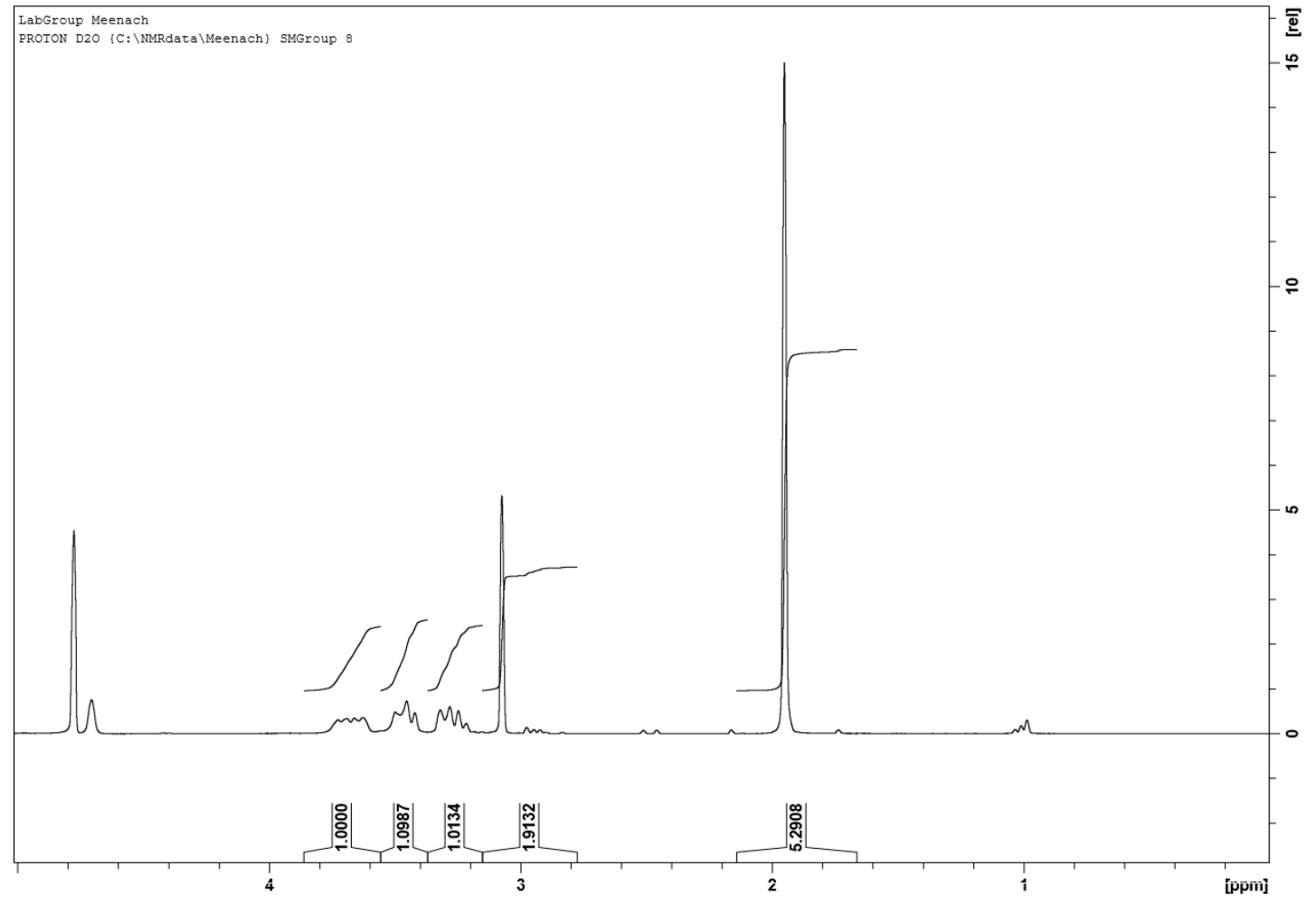

Figure A2. NMR Spectra of Ac-Dex with normalization integrations relating to associated peaks of methanol, acetone, and carbon ring of dextran. 


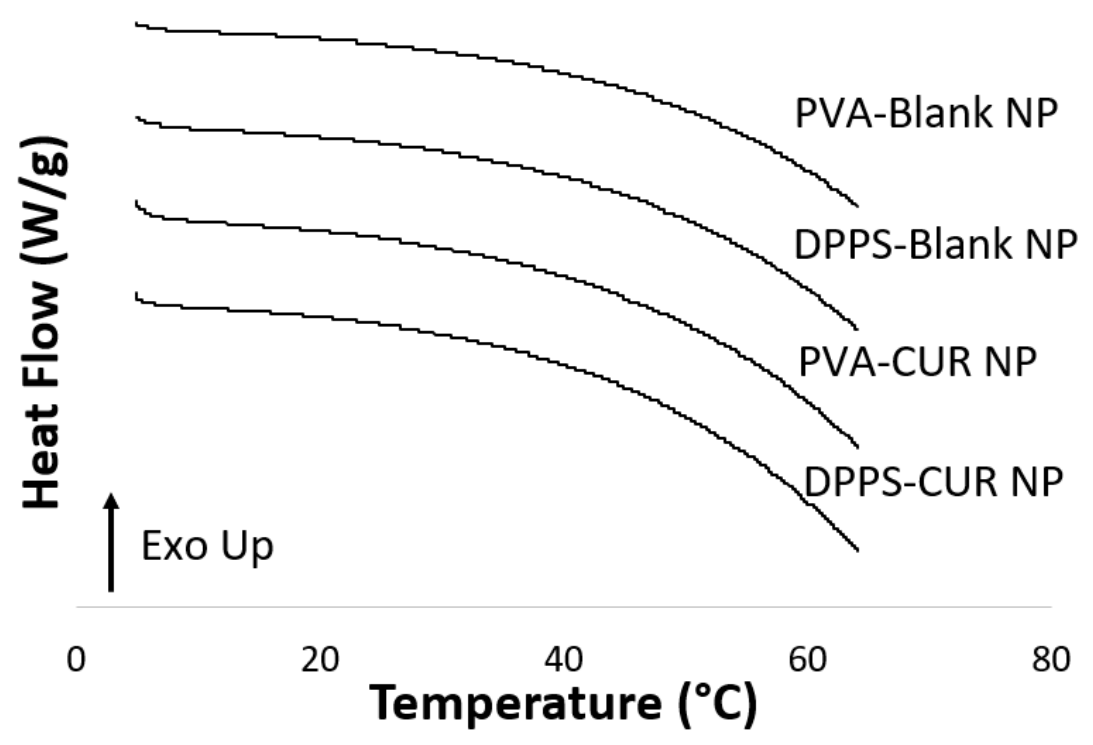

Figure A3. Wet state DSC of the nanoparticle systems. 


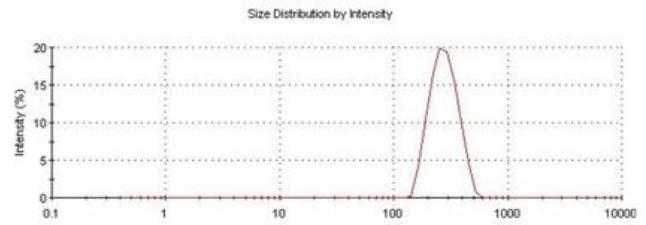

B
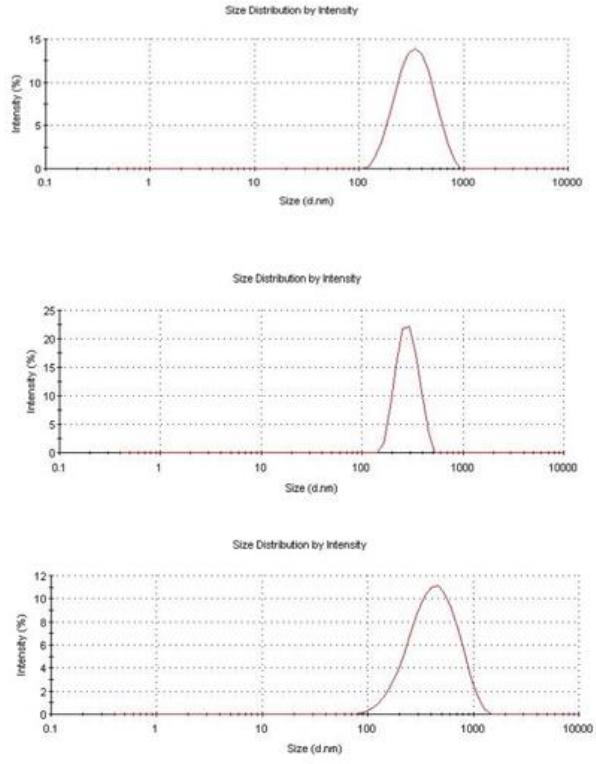

D
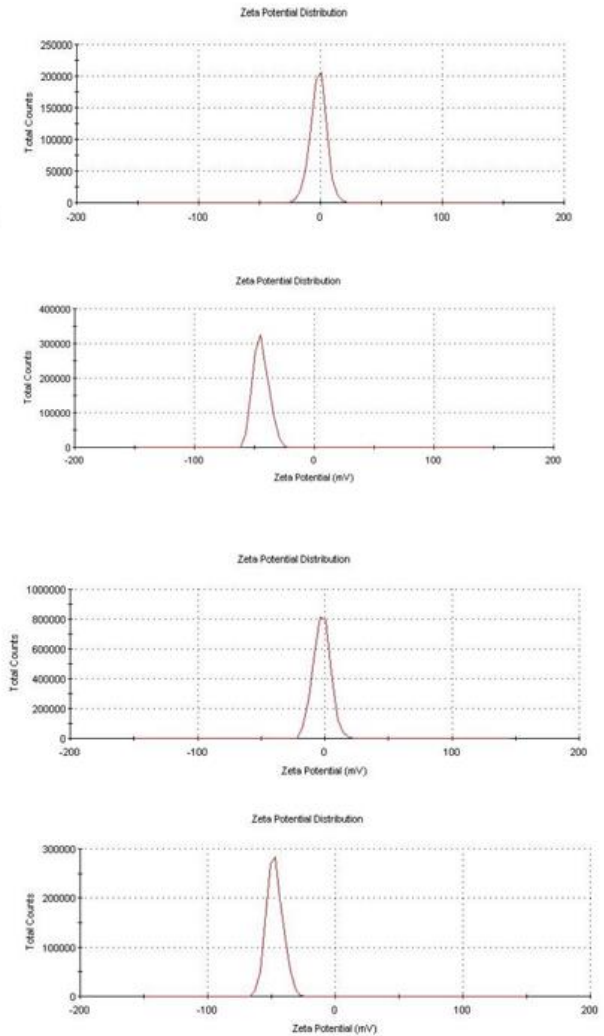

Figure A4. Diameter (left) and zeta potential (right) of Blank-PVA nanoparticle (A), Blank-DPPS nanoparticle (B), CUR-PVA nanoparticle (C), and CUR-DPPS nanoparticle (D) systems. 


\section{APPENDIX B}

Supplementary Material for Chapter 4 


\section{B.1 Materials}

The following chemicals were used: dextran from Leuconostoc mesenteroides (9,000-11,000 MW), pyridinium p-toluenesulfonate (PPTS, $\geq 99.9 \%$ ), 2methoxypropene (2-MOP, $\geq 97 \%$ ), triethylamine (TEA, $\geq 99 \%$ ), anhydrous dimethyl sulfoxide (DMSO, $\geq 99.9 \%$ ), deuterium chloride ( $\mathrm{DCl}, 35 \mathrm{wt} \%$ in $\mathrm{D}_{2} \mathrm{O}, \geq 99 \%$ atom D), sodium acetate $(\geq 99 \%)$, acetic acid $(1.0 \mathrm{~N})$, chloroform $(\geq 99 \%)$, anhydrous methanol ( $\geq 99.9 \%)$, and isopropanol ( $\geq 99 \%$ ), sulforhodamine B sodium salt (SRB), Hydranal $^{\circledR}$ KF reagent, and deuterium oxide $\left(\mathrm{D}_{2} \mathrm{O}, 99.8 \%\right.$ atom D). All cell culture materials were purchased from American Type Culture Collection (ATCC, Manassas, VA, USA), Caisson Labs (Smithfield, UT, USA), or Fisher Scientific.

\section{B.1.1 Kinetics and Release Models}

The zero-order model describes the constant, controlled release of drug from a drug formulation, disregarding changes in equilibrium and erosion of the formulation (286). Ideal delivery of drugs would follow zero-order kinetics to keep blood levels constant. The zero-order model is described by:

$$
M_{t}=K_{0} t+M_{0}
$$

where $\mathrm{M}_{\mathrm{t}}$ is the mass of drug released at time $\mathrm{t}, \mathrm{M}_{0}$ is the initial mass of drug released at $\mathrm{t}=0$, and $\mathrm{K}_{0}$ is the zero-order release rate constant. 
The first-order model describes drug release profiles as proportional to the amount of unreleased drug remaining at time, $t$, thereby indicating sustained release of the drug (286), as described by:

$$
\log C_{t}=\log C_{0}+\frac{K_{1}}{2.303} t
$$

where $\mathrm{C}_{\mathrm{t}}$ is the concentration of drug at time $\mathrm{t}, \mathrm{C}_{0}$ is the initial drug concentration at $\mathrm{t}$ $=0$, and $\mathrm{K}_{1}$ is the first-order release rate constant.

The Higuchi model describes drug release profiles based on square root time dependence (286) as seen in:

$$
\frac{M_{t}}{M}=K_{H} t^{1 / 2}
$$

where $M_{t} / M_{\infty}$ is fraction of drug released at time $t$ and $K_{H}$ is the Higuchi dissolution constant.

The Korsmeyer-Peppas (K-P) model (a.k.a. Ritger-Peppas model) is a semiempirical model that exponentially relates the amount of drug released with time (286) as seen in:

$$
\frac{M_{t}}{M}=K_{K P} t^{n}
$$


where $M_{t} / M_{\infty}$ is fraction of drug released at time $t, K_{K P}$ is the Korsmeyer-Peppas release rate constant that characterizes the structural and geometric properties of the particles, and $\mathrm{n}$ is the drug release exponent indicating the drug release mechanism. The K-P model allows for the understanding of the type of dissolution mechanism occurring during degradation, where an $n$ value of 0.43 indicates Fickian diffusion, $n$ of 0.85 indicates case II transport (erosion), and $0.43<\mathrm{n}<0.85$ indicates anomalous drug transport.

The Corrigan model is used to model burst release kinetics by combining contributions from first-order kinetics and polymer degradation, where release of drug molecules close to the surface of the system and polymer degradation are both contributions to release $(234,235)$.

$$
Q=\frac{M_{t}}{M}=F_{B, i n}\left(1 \exp \left(K_{C} t\right)\right)
$$

where $Q$ is the fraction of drug released at time $t, F_{B, i n}$ is the fraction of drug released during burst, and $\mathrm{K}_{\mathrm{C}}$ is the first-order Corrigan rate constant associated with the kinetics of the burst release (227).

The Peppas-Sahlin model $(236,237)$ accounts for both Fickian diffusion (first term in equation) and case II relaxation (second term in equation) release contributions:

$$
\frac{M_{t}}{M}=k_{1} t^{m}+k_{2} t^{2 m}
$$


where $M_{t} / M_{\infty}$ is fraction of drug released at time $t, k_{1}$ and $k_{2}$ are the kinetic constants, and $\mathrm{m}$ is the diffusional exponent. The ratio of relaxation versus Fickian contributions can be calculated from:

$$
\frac{R}{F}=\frac{k_{2}}{k_{1}} t^{m}
$$

where $\mathrm{R} / \mathrm{F}=1$ indicates a release mechanism contains both erosion and diffusion equally, $\mathrm{R} / \mathrm{F}>1$ indicates that relaxation (erosion) dominates, and $\mathrm{R} / \mathrm{F}<1$ indicates that diffusion dominates. 
Table B1. Geometric diameter and zeta potential of microparticles systems (mean \pm standard deviation, $\mathrm{n}=3$ ).

\begin{tabular}{|l|c|c|}
\hline System & Geometric & Zeta Potential \\
& diameter $(\mathbf{d g})(\boldsymbol{\mu m})$ & $(\mathbf{m V})$ \\
\hline Fast- $100^{\circ} \mathrm{C}-\mathrm{MedP}$ & $1.13 \pm 0.56$ & $-16.1 \pm 2.3$ \\
\hline Fast- $150^{\circ} \mathrm{C}-\mathrm{MedP}$ & $0.53 \pm 0.19$ & $-15.9 \pm 1.8$ \\
\hline Fast- $100^{\circ} \mathrm{C}-\mathrm{HighP}$ & $1.11 \pm 0.45$ & $-13.5 \pm 3.3$ \\
\hline Slow- $100^{\circ} \mathrm{C}-\mathrm{MedP}$ & $1.15 \pm 0.51$ & $-15.0 \pm 1.5$ \\
\hline Slow- $150^{\circ} \mathrm{C}-\mathrm{MedP}$ & $1.01 \pm 0.51$ & $-15.9 \pm 3.3$ \\
\hline Slow- $100^{\circ} \mathrm{C}-\mathrm{HighP}$ & $0.556 \pm 0.24$ & $-17.6 \pm 1.8$ \\
\hline Man- $100^{\circ} \mathrm{C}$-LowP & $3.53 \pm 2.3$ & --- \\
\hline
\end{tabular}


Table B2. Values for ratio of relaxation versus Fickian contribution (R/F) of PeppasSahlin drug release kinetics model for microparticle drug release at $\mathrm{pH}$ 5. F100M = Fast $-100^{\circ} \mathrm{C}-\mathrm{MedP}, \mathrm{F} 150 \mathrm{M}=$ Fast $-150^{\circ} \mathrm{C}-\mathrm{MedP}, \mathrm{F} 100 \mathrm{H}=$ Fast $-100^{\circ} \mathrm{C}-\mathrm{HighP}, \mathrm{S} 100 \mathrm{M}$ $=$ Slow $-100^{\circ} \mathrm{C}-\mathrm{MedP}, \mathrm{S} 150 \mathrm{M}=\mathrm{Slow}-150^{\circ} \mathrm{C}-\mathrm{MedP}, \mathrm{S} 100 \mathrm{H}=\mathrm{Slow}-100^{\circ} \mathrm{C}-\mathrm{HighP}$.

\begin{tabular}{|c|c|c|c|c|c|c|}
\hline Time (hour) & F100M & F150M & F100H & S100M & S150M & S100H \\
\hline 0 & 0 & 0 & 0 & 0 & 0 & 0 \\
\hline 1 & 0.2418 & 0.1931 & 0.1874 & 0.3134 & 0.6070 & 0.2364 \\
\hline 2 & 0.2575 & 0.1719 & 0.1967 & 0.3277 & 0.6370 & 0.2461 \\
\hline 4 & 0.2743 & 0.1813 & 0.2064 & 0.3426 & 0.6684 & 0.2562 \\
\hline 6 & 0.2847 & 0.1970 & 0.2123 & 0.3517 & 0.6874 & 0.2624 \\
\hline 8 & 0.2922 & 0.1911 & 0.2167 & 0.3582 & 0.7013 & 0.2667 \\
\hline 24 & 0.3230 & 0.2078 & 0.2340 & 0.3844 & 0.7569 & 0.2843 \\
\hline
\end{tabular}




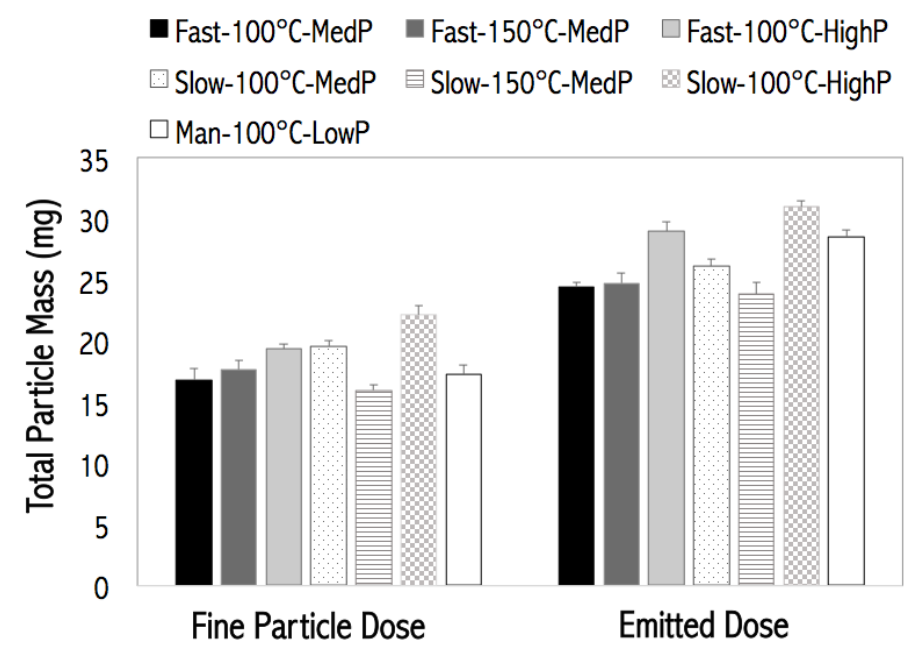

Figure B1. Total particle mass (mg) of aerosolization performance properties of particles, including fine particle fraction (FPF), respirable fraction (RF), and emitted dose (ED) (mean \pm standard deviation, $\mathrm{n}=3$ ). 


\section{APPENDIX C}

Supplementary Material for Chapter 5 


\section{C.1. Physical characteristics of drug molecules}

The physical characteristics of PTX, TAC, and RSV were acquired from www.drugbank.ca (accessed August 28th, 2018) (University of Alberta and The Metabolomics Innovation Centre, Edmonton, Alberta, Canada) or www.acdlabs.com (accessed August 30th, 2018) (Toronto, Ontario, Canada) and their chemical characteristics and molecular structures are shown in Table C1.

Table C1. Chemical structures and characteristics of the encapsulated model therapeutics paclitaxel (PTX), tacrolimus (TAC), and resveratrol (RSV).

Characteristics include molecular weight (MW), partition coefficient (Log P), and water solubility. Predictions for characteristics were calculated using ACDLabs.com and retrieved from drugbank.ca.

\begin{tabular}{|c|c|c|c|}
\hline Characteristics & Paclitaxel (PTX) & Tacrolimus (TAC) & Resveratrol (RSV) \\
\hline $\begin{array}{c}\text { Chemical } \\
\text { Structure }\end{array}$ & 853.9 & 804.0 & 228.2 \\
\hline MW (g/mol) & 3.54 & 5.59 & 3.40 \\
\hline $\begin{array}{c}\text { Log P } \\
\text { Solubility } \\
(\boldsymbol{\mu g} / \mathbf{m L})\end{array}$ & 5.56 & 4.02 & 68.80 \\
\hline
\end{tabular}




\section{C.2 Ultra performance liquid chromatography (UPLC) analysis}

The quantification of PTX, TAC, and RSV was conducted using UPLC (LaChrom, Hitachi, Japan). A C18 column $(5 \mu \mathrm{m}, 150 \mathrm{~mm} \times 4.6 \mathrm{~mm}$, Ascentis, St. Louis, MO) was used at a flow rate of $1 \mathrm{~mL} / \mathrm{min}$. For PTX and TAC content determination, the mobile phase consisted of 70\% (v/v) acetonitrile (ACN) and 30\% (v/v) aqueous phosphoric acid $(0.1 \%, \mathrm{v} / \mathrm{v})$ and the column temperature was set at $50^{\circ} \mathrm{C}$. The UV wavelengths were $227 \mathrm{~nm}$ and $215 \mathrm{~nm}$, respectively. For RSV content determination, the mobile phase was 50\% $\mathrm{ACN}$ and 50\% water containing $0.1 \%$ trifluoroacetic acid. The column temperature was set at room temperature and the UV wavelength was set at $306 \mathrm{~nm}$. 


\section{BIBLIOGRAPHY}

1. Bobo D, Robinson KJ, Islam J, Thurecht KJ, Corrie SR (2016) NanoparticleBased Medicines: A Review of FDA-Approved Materials and Clinical Trials to Date. Pharm Res 33(10):2373-2387.

2. Kamaly N, Yameen B, Wu J, Farokhzad OC (2016) Degradable controlledrelease polymers and polymeric nanoparticles: Mechanisms of controlling drug release. Chem Rev 116(4):2602-2663.

3. Loira-Pastoriza C, Todoroff J, Vanbever R (2014) Delivery strategies for sustained drug release in the lungs. Adv Drug Deliv Rev 75:81-91.

4. Mohyeldin SM, Mehanna MM, Elgindy NA (2016) The relevancy of controlled nanocrystallization on rifampicin characteristics and cytotoxicity. Int $J$ Nanomedicine 11:2209-2222.

5. Junghanns JUAH, Müller RH (2008) Nanocrystal technology, drug delivery and clinical applications. Int J Nanomedicine 3(3):295-309.

6. Bannunah AM, Vllasaliu D, Lord J, Stolnik S (2014) Mechanisms of nanoparticle internalization and transport across an intestinal epithelial cell model: effect of size and surface charge. Mol Pharm 11(12):4363-4373.

7. Li M, et al. (2018) Enhanced Synergism of Thermo-chemotherapy For Liver Cancer with Magnetothermally Responsive Nanocarriers. Theranostics 8(3):693-709.

8. Tyagi P, Subramony JA (2018) Nanotherapeutics in oral and parenteral drug delivery: Key learnings and future outlooks as we think small. J Control Release 272(December 2017):159-168. 
9. Wu B, et al. (2017) MRI-guided targeting delivery of doxorubicin with reduction-responsive lipid-polymer hybrid nanoparticles. Int J Nanomedicine $12: 6871-6882$.

10. Geelen T, et al. (2012) Internalization of paramagnetic phosphatidylserinecontaining liposomes by macrophages. J Nanobiotechnology 10(1):37.

11. Jiang SP, et al. (2013) Preparation and characteristics of lipid nanoemulsion formulations loaded with doxorubicin. Int J Nanomedicine 8:3141-3150.

12. Romeo C, et al. (2019) Gynecologic Oncology Non-pegylated liposomal doxorubicin ( NPLD, Myocet $\left.{ }^{\circledR}\right)+$ carboplatin in patients with platinum sensitive ovarian cancers : A ARCAGY- GINECO phase IB-II trial. Gynecol Oncol 152(1):68-75.

13. Rafiyath SM, et al. (2012) Comparison of safety and toxicity of liposomal doxorubicin vs . conventional anthracyclines : a meta-analysis. Exp Hematol Oncol 1(10):1-9.

14. Yang X, Yang M, Pang B, Vara M, Xia Y (2015) Gold Nanomaterials at Work in Biomedicine. Chem Rev 115(19):10410-10488.

15. Laurent S, et al. (2008) Magnetic Iron Oxide Nanoparticles: Synthesis, Stabilization, Vectorization, Physicochemical Characterizations, and Biological Applications (vol 108, pg 2064, 2008). Chem Rev 108(6):2064-2110.

16. Dissanayake S, Denny WA, Gamage S, Sarojini V (2017) Recent developments in anticancer drug delivery using cell penetrating and tumor targeting peptides. J Control Release 250:62-76.

17. Joubert N, Denevault-Sabourin C, Bryden F, Viaud-Massuard MC (2017) 
Towards antibody-drug conjugates and prodrug strategies with extracellular stimuli-responsive drug delivery in the tumor microenvironment for cancer therapy. Eur J Med Chem 142:393-415.

18. Gu M, Wang X, Toh TB, Chow EK-H (2017) Applications of stimuliresponsive nanoscale drug delivery systems in translational research. Drug Discov Today 00(00). doi:10.1016/j.drudis.2017.11.009.

19. Bachelder EM, Pino EN, Ainslie KM (2017) Acetalated Dextran: A Tunable and Acid-Labile Biopolymer with Facile Synthesis and a Range of Applications. Chem Rev 117(3):1915-1926.

20. Baumgartner R, et al. (2014) Nano-extrusion : A promising tool for continuous manufacturing of solid nano-formulations. Int J Pharm 477(1-2):1-11.

21. Sayes CM, Aquino G V, Hickey AJ (2017) Nanomaterial Drug Products : Manufacturing and Analytical Perspectives. AAPS J 19(1):18-25.

22. Worsham RD, Thomas V, Farid SS (2019) Potential of Continuous Manufacturing for Liposomal Drug Products. Biotechnol J 1700740(14):1-8.

23. Musumeci T, Leonardi A, Bonaccorso A, Pignatello R, Puglisi G (2018) Tangential Flow Filtration Technique: An Overview on Nanomedicine Applications. Pharm Nanotechnol. doi:10.2174/2211738506666180306160921.

24. Dalwadi G, Benson HAE, Chen Y (2005) Comparison of diafiltration and tangential flow filtration for purification of nanoparticle suspensions. Pharm Res 22(12):2152-2162.

25. Limayem I, Charcosset C, Fessi H (2004) Purification of nanoparticle suspensions by a concentration/diafiltration process. Sep Purif Technol 38(1):1- 
9.

26. Heydenreich A V., Westmeier R, Pedersen N, Poulsen HS, Kristensen HG (2003) Preparation and purification of cationic solid lipid nanospheres - Effects on particle size, physical stability and cell toxicity. Int J Pharm 254(1):83-87.

27. Morrison MA, Benoit G (2004) Investigation of conventional membrane and tangential flow ultrafiltration artifacts and their application to the characterization of freshwater colloids. Environ Sci Technol 38(24):6817-6823.

28. Sweeney SF, Woehrle GH, Hutchison JE (2006) Rapid purification and size separation of gold nanoparticles via diafiltration. J Am Chem Soc 128(10):3190-3197.

29. Hirsjärvi S, Peltonen L, Hirvonen J (2009) Effect of Sugars, Surfactant, and Tangential Flow Filtration on the Freeze-Drying of Poly(lactic acid) Nanoparticles. AAPS PharmSciTech 10(2):488-494.

30. Hirsjärvi S, Qiao Y, Royere A, Bibette J, Benoit JP (2010) Layer-by-layer surface modification of lipid nanocapsules. Eur J Pharm Biopharm 76(2):200207.

31. Mihaila R, et al. (2011) Lipid nanoparticle purification by Spin CentrifugationDialysis (SCD): A facile and high-throughput approach for small scale preparation of siRNA-lipid complexes. Int J Pharm 420(1):118-121.

32. Satzer P, Wellhoefer M, Jungbauer A (2014) Continuous separation of protein loaded nanoparticles by simulated moving bed chromatography. J Chromatogr A 1349:44-49.

33. Heinemann ML, et al. (2014) Benchtop isolation and characterization of 
functional exosomes by sequential filtration. J Chromatogr A 1371:125-135.

34. Björnmalm M, et al. (2015) Flow-Based Assembly of Layer-by-Layer Capsules through Tangential Flow Filtration. Langmuir 31(33):9054-9060.

35. Chen N, et al. (2016) Degradation of acetalated dextran can be broadly tuned based on cyclic acetal coverage and molecular weight. Int J Pharm 512(1):147157.

36. Broaders KE, Cohen J a, Beaudette TT, Bachelder EM, Fréchet JMJ (2009) Acetalated dextran is a chemically and biologically tunable material for particulate immunotherapy. Proc Natl Acad Sci U S A 106(14):5497-502.

37. Wahyu D, Kumar A, Dhawan G, Mishra D (2018) Therapeutic inhibition of spleen tyrosine kinase in in fl ammatory macrophages using PLGA nanoparticles for the treatment of non-alcoholic steatohepatitis. J Control Release 288(September):227-238.

38. Chachuli SHM, Nawaz A, Shah K, Naharudin I, Wong TW (2016) In Vitro Investigation of Influences of Chitosan Nanoparticles on Fluorescein Permeation into Alveolar Macrophages. Pharm Res 33(6):1497-1508.

39. Gupta N, Ibrahim HM, Ahsan F (2014) Peptide-Micelle Hybrids Containing Fasudil for Targeted Delivery to the Pulmonary Arteries and Arterioles to Treat Pulmonary Arterial Hypertension. J Pharm Sci 103(11):3743-3753.

40. Bachelder EM, Beaudette TT, Broaders KE, Dashe J, Fréchet JMJ (2008) Acetal-derivatized dextran: An acid-responsive biodegradable material for therapeutic applications. J Am Chem Soc 130(32):10494-10495.

41. Collier MA, et al. (2016) Host-mediated Leishmania donovani treatment using 
AR-12 encapsulated in acetalated dextran microparticles. Int J Pharm 499(12):186-194.

42. Peine KJ, et al. (2014) Treatment of experimental autoimmune encephalomyelitis by codelivery of disease associated peptide and dexamethasone in acetalated dextran microparticles. Mol Pharm 11(3):828835.

43. Kauffman KJ, et al. (2012) Optimization of rapamycin-loaded acetalated dextran microparticles for immunosuppression. Int J Pharm 422(1-2):356-363.

44. Wang Z, Meenach SA (2017) Optimization of Acetalated Dextran-Based Nanocomposite Microparticles for Deep Lung Delivery of Therapeutics via Spray-Drying. J Pharm Sci:1-9.

45. Wang Z, Gupta SK, Meenach SA (2017) Development and physicochemical characterization of acetalated dextran aerosol particle systems for deep lung delivery. Int J Pharm 525(1):264-274.

46. Gause KT, et al. (2016) Immunological Principles Guiding the Rational Design of Particles for Vaccine Delivery. (i). doi:10.1021/acsnano.6b07343.

47. Chen N, et al. (2018) Tunable degradation of acetalated dextran microparticles enables controlled vaccine adjuvant and antigen delivery to modulate adaptive immune responses. J Control Release 273(January):147-159.

48. Kanthamneni N, et al. (2012) Enhanced stability of horseradish peroxidase encapsulated in acetalated dextran microparticles stored outside cold chain conditions. Int J Pharm 431(1-2):101-110.

49. Meenach S a, et al. (2012) Synthesis, Optimization, and Characterization of 
Camptothecin- Loaded Acetalated Dextran Porous Microparticles for Pulmonary Delivery. Mol Pharm. doi:10.1021/mp2003785.

50. Graham-Gurysh E, et al. (2018) Sustained Delivery of Doxorubicin via Acetalated Dextran Scaffold Prevents Glioblastoma Recurrence after Surgical Resection. Mol Pharm 15(3):1309-1318.

51. Hoang K V., et al. (2014) Acetalated dextran encapsulated AR-12 as a hostdirected therapy to control Salmonella infection. Int J Pharm 477(1-2):334343.

52. Shah NK, Gupta SK, Wang Z, Meenach SA (2019) Enhancement of macrophage uptake via phosphatidylserine-coated acetalated dextran nanoparticles. J Drug Deliv Sci Technol 50(December 2018):57-65.

53. Wang Z, Cuddigan JL, Gupta SK, Meenach SA (2016) Nanocomposite microparticles (nCmP) for the delivery of tacrolimus in the treatment of pulmonary arterial hypertension. Int J Pharm 512(1):305-313.

54. Wang Z, Meenach SA (2016) Synthesis and Characterization of Nanocomposite Microparticles (nCmP) for the Treatment of Cystic Fibrosis-Related Infections. Pharm Res:1-11.

55. Shah NK, Torrico EA, Wang Z, Meenach SA (2019) Nanoparticles for Biomedical Applications: Fundamental Concepts, Body Interactions, and Clinical Potential. Chapter 6: Routes of Administration eds Chung EJ, Leon L, Rinaldi C (Elsevier Science \& Technology; Amsterdam, The Netherlands).

56. Othmer K (2002) Encyclopedia of Chemical Technology (John Wiley \& Sons Inc.). 
57. Albanese A, Tang PS, Chan WCW (2012) The Effect of Nanoparticle Size, Shape, and Surface Chemistry on Biological Systems. Annu Rev Biomed Eng. doi:10.1146/annurev-bioeng-071811-150124.

58. Citrome L (2010) Paliperidone palmitate - review of the efficacy, safety and cost of a new second-generation depot antipsychotic medication. Int J Clin Pract 64(2):216-239.

59. Wang Y, Qu W, Choi S (2016) FDA's Regulatory Science Program for Generic PLA/ PLGA-Based Drug Products. 1.

60. Haynes A, et al. (2016) Inhalation of tobramycin using simulated cystic fibrosis patient profiles. Pediatr Pulmonol 51(11):1159-1167.

61. PH H, et al. (2002) In vivo lung deposition of hollow porous particles from a pressurized metered dose inhaler. Pharm Res 19(3):258-264.

62. Newswire CP (2018) U.S. FDA Clears Sebacia Microparticles for the Treatment of Mild to Moderate Inflammatory Acne. Sebacia, Inc.

63. Paithankar DY, et al. (2015) Acne Treatment Based on Selective Photothermolysis of Sebaceous Follicles with Topically Delivered. J Investig Dermatology 135:1727-1734.

64. Jia Z, Wang W (2018) Yttrium-90 radioembolization for unresectable metastatic neuroendocrine liver tumor: A systematic review. Eur J Radiol 100(September 2017):23-29.

65. Hulla JE, Sahu SC, Hayes AW (2015) Nanotechnology : History and future. Hum Exp Toxicol 34(12):1318-1321.

66. Havel HA (2016) Where Are the Nanodrugs? An Industry Perspective on 
Development of Drug Products Containing Nanomaterials. AAPS J 18(6):13511353.

67. Shi Y, Li L (2005) Current advances in sustained-release systems for parenteral drug delivery. Expert Opin Drug Deliv 2(6):1039-1058.

68. Gulati N, Gupta H (2011) Parenteral Drug Delivery : A Review. 133-145.

69. Teekamp N, Duque LF, Frijlink HW, Hinrichs WL, Olinga P (2015) Production methods and stabilization strategies for polymer-based nanoparticles and microparticles for parenteral delivery of peptides and proteins. Expert Opin Drug Deliv 12(8):1311-1331.

70. Luo C, Sun J, Du Y, He Z (2014) Emerging integrated nanohybrid drug delivery systems to facilitate the intravenous-to-oral switch in cancer chemotherapy. J Control Release 176:94-103.

71. Reddy LH, Bazile D (2014) Drug delivery design for intravenous route with integrated physicochemistry, pharmacokinetics and pharmacodynamics: Illustration with the case of taxane therapeutics. Adv Drug Deliv Rev 71:34-57.

72. Almeida AJ, Souto E (2007) Solid lipid nanoparticles as a drug delivery system for peptides and proteins. Adv Drug Deliv Rev 59(6):478-490.

73. Gamazo C, et al. (2007) Biodegradable gentamicin delivery systems for parenteral use for the treatment of intracellular bacterial infections. Expert Opin Drug Deliv 4(6):677-688.

74. Ahmed TA, Aljaeid BM (2016) Preparation, characterization, and potential application of chitosan, chitosan derivatives, and chitosan metal nanoparticles in pharmaceutical drug delivery. Drug Des Devel Ther 10(January):483-507. 
75. de Souza Carvalho C, Daum N, Lehr CM (2014) Carrier interactions with the biological barriers of the lung: Advanced in vitro models and challenges for pulmonary drug delivery. Adv Drug Deliv Rev 75:129-140.

76. Fornaguera C, et al. (2015) Interactions of PLGA nanoparticles with blood components: Protein adsorption, coagulation, activation of the complement system and hemolysis studies. Nanoscale. doi:10.1039/c5nr00733j.

77. Anselmo AC, Mitragotri S (2016) Nanoparticles in the clinic. Bioeng Transl Med 1(1):10-29.

78. Rafiei P, Haddadi A (2017) Docetaxel-loaded PLGA and PLGA-PEG nanoparticles for intravenous application: Pharmacokinetics and biodistribution profile. Int J Nanomedicine. doi:10.2147/IJN.S121881.

79. Honary S, Zahir F (2013) Effect of zeta potential on the properties of nano-drug delivery systems - A review (Part 1). Trop J Pharm Res 12(2):255-264.

80. Honary S, Zahir F (2013) Effect of Zeta Potential on the Properties of Nano Drug Delivery Systems - A Review (Part 2). Trop J Pharm al Res 12(2):265273.

81. Qie Y, et al. (2016) Surface modification of nanoparticles enables selective evasion of phagocytic clearance by distinct macrophage phenotypes. Sci Rep 6(April):26269.

82. Bagalkot V, Deiuliis JA, Rajagopalan S, Maiseyeu A (2016) "Eat me” imaging and therapy. Adv Drug Deliv Rev 99:2-11.

83. Suk JS, Xu Q, Kim N, Hanes J, Ensign LM (2016) PEGylation as a strategy for improving nanoparticle-based drug and gene delivery. Adv Drug Deliv Rev 
99:28-51.

84. Gratton SEA, et al. (2008) The effect of particle design on cellular internalization pathways. Proc Natl Acad Sci U S A 105(33).

85. Ajorlou E, Khosroushahi AY (2017) Trends on polymer- and lipid-based nanostructures for parenteral drug delivery to tumors. Cancer Chemother Pharmacol 79(2):251-265.

86. Xi A, Bothun GD (2014) Centrifugation-based assay for examining nanoparticle-lipid membrane binding and disruption. Analyst. doi:10.1039/c3an01601c.

87. Ventola CL (2017) Progress in Nanomedicine: Approved and Investigational Nanodrugs. $P$ T 42(12):742-755.

88. Gupta N, Hatoum H, Dy GK (2014) First line treatment of advanced non-smallcell lung cancer - specific focus on albumin bound paclitaxel. Int $J$ Nanomedicine 9:209-21.

89. Oliveira CL, et al. (2017) Characterization of polymeric nanoparticles for intravenous delivery: Focus on stability. Colloids Surfaces B Biointerfaces. doi:10.1016/j.colsurfb.2016.10.046.

90. Hunt H, et al. (2017) Targeting of p32 in peritoneal carcinomatosis with intraperitoneal linTT1 peptide-guided pro-apoptotic nanoparticles. J Control Release 260(June):142-153.

91. Colby AH, et al. (2017) Nanoparticle drug-delivery systems for peritoneal cancers: a case study of the design, characterization and development of the expansile nanoparticle. Wiley Interdiscip Rev Nanomedicine 
Nanobiotechnology 9(3):1-20.

92. Dakwar GR, et al. (2014) Colloidal stability of nano-sized particles in the peritoneal fluid: Towards optimizing drug delivery systems for intraperitoneal therapy. Acta Biomater. doi:10.1016/j.actbio.2014.03.012.

93. Nowacki M, et al. (2017) Nanoparticle as a novel tool in hyperthermic intraperitoneal and pressurized intraperitoneal aerosol chemotheprapy to treat patients with peritoneal carcinomatosis. Oncotarget.

doi:10.18632/oncotarget.20596.

94. Xu S, et al. (2016) Thermosensitive hydrogel system assembled by PTX-loaded copolymer nanoparticles for sustained intraperitoneal chemotherapy of peritoneal carcinomatosis. Eur J Pharm Biopharm 104:251-259.

95. Williamson SK, et al. (2015) A phase i study of intraperitoneal nanoparticulate paclitaxel $\left(\operatorname{Nanotax}{ }^{\circledR}\right)$ in patients with peritoneal malignancies. Cancer Chemother Pharmacol 75(5):1075-1087.

96. Sun B, et al. (2016) Intraperitoneal chemotherapy of ovarian cancer by hydrogel depot of paclitaxel nanocrystals. J Control Release 235:91-98.

97. Deng Y, et al. (2016) Improved i.p. drug delivery with bioadhesive nanoparticles. Proc Natl Acad Sci 113(41):11453-11458.

98. Dasa SSK, et al. (2018) Plectin-targeted liposomes enhance the therapeutic efficacy of a PARP inhibitor in the treatment of ovarian cancer. Theranostics. doi:10.7150/thno.23050.

99. Wu C-C, et al. (2015) Nanoparticle-induced intraperitoneal hyperthermia and targeted photoablation in treating ovarian cancer. Oncotarget 6(29):26861- 
26875 .

100. Ye H, Karim AA, Loh XJ (2015) Current treatment options and drug delivery systems as potential therapeutic agents for ovarian cancer: A review. Mater Sci Eng C 45:609-619.

101. MIKOLAJCZYK A, et al. (2018) Particle Stability During Pressurized Intraperitoneal Aerosol Chemotherapy (PIPAC). Anticancer Res 38(8):4645-4649.

102. Solaß W, Giger-Pabst U, Zieren J, Reymond MA (2013) Pressurized intraperitoneal aerosol chemotherapy (PIPAC): Occupational health and safety aspects. Ann Surg Oncol 20(11):3504-3511.

103. Bagley AF, Hill S, Rogers GS, Bhatia SN (2013) Plasmonic photothermal heating of intraperitoneal tumors through the use of an implanted near-infrared source. ACS Nano 7(9):8089-8097.

104. Vaccine Recommendations and Guidelines of the ACIP https://www.cdc.gov/vaccines/hcp/acip-recs/general-recs/administration.html.

105. Din F ud, et al. (2017) Irinotecan-loaded double-reversible thermogel with improved antitumor efficacy without initial burst effect and toxicity for intramuscular administration. Acta Biomater. doi:10.1016/j.actbio.2017.03.007.

106. Wright L, Rao S, Thomas N, Boulos RA, Prestidge CA (2018) Ramizol@encapsulation into extended release PLGA micro- and nanoparticle systems for subcutaneous and intramuscular administration: in vitro and in vivo evaluation. Drug Dev Ind Pharm 44(9):1451-1457.

107. Gonzalez-Aramundiz JV, Peleteiro Olmedo M, Gonzalez-Fernandez A, Alonso Fernandez MJ, Csaba NS (2015) Protamine-based nanoparticles as new antigen 
delivery systems. Eur J Pharm Biopharm 97:51-59.

108. Dewangan HK, Pandey T, Maurya L, Singh S (2018) Rational design and evaluation of HBsAg polymeric nanoparticles as antigen delivery carriers. Int J Biol Macromol 111:804-812.

109. Darville N, et al. (2016) Modeling the Time Course of the Tissue Responses to Intramuscular Long-acting Paliperidone Palmitate Nano-/Microcrystals and Polystyrene Microspheres in the Rat. Toxicol Pathol 44(2):189-210.

110. Xie B, et al. (2018) Progesterone PLGA/mPEG-PLGA Hybrid Nanoparticle Sustained-Release System by Intramuscular Injection. Pharm Res. doi:10.1007/s11095-018-2357-X.

111. Darville N, et al. (2014) Intramuscular administration of paliperidone palmitate extended-release injectable microsuspension induces a subclinical inflammatory reaction modulating the pharmacokinetics in rats. J Pharm Sci 103(7):20722087.

112. Caputo A, et al. (2009) Induction of humoral and enhanced cellular immune responses by novel core-shell nanosphere- and microsphere-based vaccine formulations following systemic and mucosal administration. Vaccine 27(27):3605-3615.

113. Lu F, et al. (2015) Dendrimer-like alpha-d-glucan nanoparticles activate dendritic cells and are effective vaccine adjuvants. J Control Release. doi:10.1016/j.jconrel.2015.03.002.

114. Ogston-Tuck S (2014) Subcutaneous injection technique: an evidence-based approach. Nurs Stand 29(3):53-58. 
115. McLennan DN, Porter CJH, Charman SA (2005) Subcutaneous drug delivery and the role of the lymphatics. Drug Discov Today Technol 2(1):89-96.

116. Singh Y, et al. (2016) Subcutaneously Administered Ultrafine PLGA Nanoparticles Containing Doxycycline Hydrochloride Target Lymphatic Filarial Parasites. Mol Pharm 13(6):2084-2094.

117. Trevaskis NL, Kaminskas LM, Porter CJH (2015) From sewer to saviourtargeting the lymphatic system to promote drug exposure and activity. Nat Rev Drug Discov 14(11):781-803.

118. Zhu S, Li X, Lansakara-P DSP, Kumar A, Cui Z (2013) A nanoparticle depot formulation of 4-(N)-stearoyl gemcitabine shows a strong anti-tumour activity. J Pharm Pharmacol 65(2):236-242.

119. Viola M, et al. (2018) Subcutaneous delivery of monoclonal antibodies: How do we get there? J Control Release 286(July):301-314.

120. Mandal S, et al. (2017) Tenofovir alafenamide and elvitegravir loaded nanoparticles for long-acting prevention of HIV-1 vaginal transmission. Aids 31(4):469-476.

121. Gautier JC, et al. (1992) Biodegradable nanoparticles for subcutaneous administration of growth hormone releasing factor (hGRF). J Control Release 20(1):67-77.

122. Jogala S, Chinnala KM, Aukunuru J (2016) Novel subcutaneous sustained release nanoparticles encapsulating low molecular weight heparin (LMWH): Preparation, characterization and evaluation. Int J Pharm Pharm Sci 8(6):264268. 
123. Jean M, et al. (2009) Chitosan-plasmid nanoparticle formulations for IM and SC delivery of recombinant FGF-2 and PDGF-BB or generation of antibodies. Gene Ther 16(9):1097-1110.

124. Schumann C, et al. (2018) Increasing lean muscle mass in mice via nanoparticle-mediated hepatic delivery of follistatin mRNA. Theranostics 8(19):5276-5288.

125. Nimitha Vv PSSCNVV (2017) Leflunomide loaded solid lipid nanoparticle in rheumatoid. 9(2):29681-29706.

126. Pandey R, Khuller GK (2004) Subcutaneous nanoparticle-based antitubercular chemotherapy in an experimental model. J Antimicrob Chemother 54(1):266268.

127. Turner MR, Balu-Iyer S V (2018) Challenges and Opportunities for the Subcutaneous Delivery of Therapeutic Proteins. J Pharm Sci 107(5):12471260.

128. Daniher DI, Zhu J (2008) Dry powder platform for pulmonary drug delivery. Particuology 6(4):225-238.

129. Ruge CA, Kirch J, Lehr C-M (2013) Pulmonary drug delivery: from generating aerosols to overcoming biological barriers - therapeutic possibilities and technological challenges. Lancet Respir Med 1(5):402-413.

130. Loira-pastoriza C, Todoroff J, Vanbever R (2014) Delivery strategies for sustained drug release in the lungs. Adv Drug Deliv Rev 75:81-91.

131. Bailey MM, Berkland CJ (2009) Nanoparticle formulations in pulmonary drug delivery. Med Res Rev 29(1):196-212. 
132. Ngan CL, Asmawi AA (2018) Lipid-based pulmonary delivery system: a review and future considerations of formulation strategies and limitations. Drug Deliv Transl Res 8(5):1527-1544.

133. Yang W, Peters JI, Williams RO (2008) Inhaled nanoparticles-A current review. Int J Pharm 356(1):239-247.

134. Parumasivam T, et al. (2016) In vitro evaluation of novel inhalable dry powders consisting of thioridazine and rifapentine for rapid tuberculosis treatment. Eur J Pharm Biopharm 107:205-214.

135. Meenach SA, et al. (2013) Design, physicochemical characterization, and optimization of organic solution advanced spray-dried inhalable dipalmitoylphosphatidylcholine (DPPC) and dipalmitoylphosphatidylethanolamine poly(ethylene glycol) (DPPE-PEG) microparticles and nanoparticles f. Int J Nanomedicine 8:275-293.

136. Pilcer G, Amighi K (2010) Formulation strategy and use of excipients in pulmonary drug delivery. Int J Pharm 392(1-2):1-19.

137. Mansour HM, Rhee YS, Wu X (2009) Nanomedicine in pulmonary delivery. Int J Nanomedicine 4:299-319.

138. Myrdal PB, Sheth P, Stein SW (2014) Advances in metered dose inhaler technology: formulation development. AAPS PharmSciTech 15(2):434-455.

139. Ibrahim M, Verma R, Garcia-Contreras L (2015) Inhalation drug delivery devices: technology update. Med Devices 8:131-139.

140. Callard preedy E, Prokopovich P (2013) 2 - History of inhaler devices. Inhaler Devices, ed Prokopovich P (Woodhead Publishing), pp 13-28. 
141. Ali M (2010) CHAPTER 9 - Pulmonary Drug Delivery. Handbook of NonInvasive Drug Delivery Systems, ed Kulkarni VS (William Andrew Publishing, Boston), pp 209-246.

142. Javadzadeh Y, Yaqoubi S (2017) Chapter 20 - Therapeutic nanostructures for pulmonary drug delivery. Nanostructures for Drug Delivery, eds Andronescu E, Grumezescu AM (Elsevier), pp 619-638.

143. Vogt FG, Hayes D, Mansour HM (2014) Microparticulate / Nanoparticulate Mannitol Powders. 27(2):81-93.

144. Chew N, Chan H (2001) Use of solid corrugated particles to enhance powder aerosol performance. Pharm Res 11(18):1570-7.

145. Weers JG, Miller DP (2015) Formulation Design of Dry Powders for Inhalation. J Pharm Sci 104(10):3259-3288.

146. Chew NYK, Tang P, Chan HK, Raper JA (2005) How much particle surface corrugation is sufficient to improve aerosol performance of powders? Pharm Res 22(1):148-152.

147. Muralidharan P, Malapit M, Mallory E, Hayes D, Mansour HM (2015) Inhalable nanoparticulate powders for respiratory delivery. Nanomedicine Nanotechnology, Biol Med 11(5):1189-1199.

148. Pham D-D, Fattal E, Tsapis N (2015) Pulmonary drug delivery systems for tuberculosis treatment. Int J Pharm 478(2):517-529.

149. Asmawi AA, et al. (2018) Excipient selection and aerodynamic characterization of nebulized lipid-based nanoemulsion loaded with docetaxel for lung cancer treatment. Drug Deliv Transl Res. doi:10.1007/s13346-018-0526-4. 
150. Levet V, et al. (2016) Development of controlled-release cisplatin dry powders for inhalation against lung cancers. Int J Pharm 515(1-2):209-220.

151. Khutoryanskiy V V. (2017) Beyond PEGylation: Alternative surfacemodification of nanoparticles with mucus-inert biomaterials. Adv Drug Deliv Rev 124:140-149.

152. Zhang J, Wu L, Chan H-K, Watanabe W (2011) Formation, characterization, and fate of inhaled drug nanoparticles. Adv Drug Deliv Rev 63(6):441-455.

153. Miller DP, et al. (2015) Physical Characterization of Tobramycin Inhalation Powder: I. Rational Design of a Stable Engineered-Particle Formulation for Delivery to the Lungs. Mol Pharm 12(8):2582-2593.

154. Lock JY, Carlson TL, Carrier RL (2017) Mucus models to evaluate the diffusion of drugs and particles. Adv Drug Deliv Rev 124:34-49.

155. Lai SK, Wang YY, Wirtz D, Hanes J (2009) Micro- and macrorheology of mucus. Adv Drug Deliv Rev 61(2):86-100.

156. Bansil R, Turner BS (2017) The biology of mucus: Composition, synthesis and organization. Adv Drug Deliv Rev 124:3-15.

157. Lee W-H, Loo C-Y, Traini D, Young PM (2015) Inhalation of nanoparticlebased drug for lung cancer treatment: Advantages and challenges. Asian $J$ Pharm Sci 10(6):481-489.

158. Bannunah AM, Vllasaliu D, Lord J, Stolnik S (2014) Mechanisms of nanoparticle internalization and transport across an intestinal epithelial cell model: Effect of size and surface charge. Mol Pharm 11(12):4363-4373.

159. Zhao F, et al. (2011) Cellular uptake, intracellular trafficking, and cytotoxicity 
of nanomaterials. Small 7(10):1322-1337.

160. Kuhn DA, et al. (2014) Different endocytotic uptake mechanisms for nanoparticles in epithelial cells and macrophages. Beilstein J Nanotechnol 5:1625-1636.

161. Karner S, Anne N (2011) The impact of electrostatic charge in pharmaceutical powders with specific focus on inhalation-powders. J Aerosol Sci 42(6):428445.

162. Patil JS, Sarasija S (2012) Pulmonary drug delivery strategies: A concise, systematic review. Lung India 29(1):44-49.

163. Mert O, et al. (2012) A poly ( ethylene glycol ) -based surfactant for formulation of drug-loaded mucus penetrating particles. J Control Release 157(3):455-460.

164. Fromen CA, et al. (2016) Nanoparticle surface charge impacts distribution, uptake and lymph node trafficking by pulmonary antigen-presenting cells. Nanomedicine 12(3):677-687.

165. Thorley AJ, Ruenraroengsak P, Potter TE, Tetley TD (2014) Critical Determinants of Uptake and Translocation of Nanoparticles by the Human Pulmonary Alveolar Epithelium. ACS Nano 8(11):11778-11789.

166. Craparo EF, Porsio B, Sardo C, Giammona G, Cavallaro G (2016) Pegylated Polyaspartamide-Polylactide-Based Nanoparticles Penetrating Cystic Fibrosis Artificial Mucus. Biomacromolecules 17(3):767-777.

167. Leal J, et al. (2018) Mucus-penetrating phage-displayed peptides for improved transport across a mucus-like model. Int J Pharm 553(1-2):57-64. 
168. Khanvilkar K, Donovan MD, Flanagan DR (2001) Drug transfer through mucus. Adv Drug Deliv Rev 48(2-3):173-193.

169. Murgia X, Loretz B, Hartwig O, Hittinger M, Lehr CM (2017) The role of mucus on drug transport and its potential to affect therapeutic outcomes. $A d v$ Drug Deliv Rev 124:82-97.

170. Bhat PG, Flanagan DR, Donovan MD (1996) Drug diffusion through cystic fibrotic mucus: Steady-state permeation, rheologic properties, and glycoprotein morphology. J Pharm Sci 85(6):624-630.

171. Xu Q, et al. (2015) Impact of Surface Polyethylene Glycol (PEG) Density on Biodegradable Nanoparticle Transport in Mucus ex Vivo and Distribution in Vivo. ACS Nano 9(9):9217-9227.

172. Sanders NN, et al. (2000) Cystic fibrosis sputum: A barrier to the transport of nanospheres. Am J Respir Crit Care Med 162(5):1905-1911.

173. Ahsan F, Rivas IP, Khan MA, Torres Suárez AI (2002) Targeting to macrophages: Role of physicochemical properties of particulate carriers Liposomes and microspheres - On the phagocytosis by macrophages. J Control Release 79(1-3):29-40.

174. Ravichandran KS (2011) Beginnings of a Good Apoptotic Meal: The Find-Me and Eat-Me Signaling Pathways. Immunity 35(4):445-455.

175. Bagalkot V, et al. (2015) Hybrid nanoparticles improve targeting to inflammatory macrophages through phagocytic signals. J Control Release 217:243-255.

176. Chachuli SHM, Nawaz A, Shah K, Naharudin I, Wong TW (2016) In Vitro 
Investigation of Influences of Chitosan Nanoparticles on Fluorescein

Permeation into Alveolar Macrophages. Pharm Res:1-12.

177. El-sherbiny IM, Smyth HDC (2012) Controlled Release Pulmonary

Administration of Curcumin Using Swellable Biocompatible Microparticles.

Mol Pharm.

178. Fadok VA, et al. (1992) Exposure of phosphatidylserine on the surface of apoptotic lymphocytes triggers specific recognition and removal by macrophages. J Immunol 148(7):2207-16.

179. Gaspar DP, et al. (2016) Rifabutin-loaded solid lipid nanoparticles for inhaled antitubercular therapy: Physicochemical and in vitro studies. Int J Pharm 497(1-2):199-209.

180. Gramatica A, et al. (2014) ??Env-decorated phosphatidylserine liposomes trigger phagocytosis of HIV-virus-like particles in macrophages. Nanomedicine Nanotechnology, Biol Med 10(5):981-989.

181. Matsumoto A, et al. (2016) Role of Phosphatidylserine-Derived Negative Surface Charges in the Recognition and Uptake of Intravenously Injected B16BL6-Derived Exosomes by Macrophages. J Pharm Sci 106(1):168-175.

182. Petazzi RA, Gramatica A, Herrmann A, Chiantia S (2015) Time-controlled phagocytosis of asymmetric liposomes: Application to phosphatidylserine immunoliposomes binding HIV-1 virus-like particles. Nanomedicine Nanotechnology, Biol Med 11(8):1985-1992.

183. Wang J, et al. (2016) Enhancement of Anti-Inflammatory Activity of Curcumin Using Phosphatidylserine-Containing Nanoparticles in Cultured Macrophages. 
Int J Mol Sci 17(6):969.

184. Zhang L, Zhou H, Belzile O, Thorpe P, Zhao D (2014) Phosphatidylserinetargeted bimodal liposomal nanoparticles for in vivo imaging of breast cancer in mice. J Control Release 183(1):114-123.

185. Zheng Y, Chen Y, Jin L, Ye H, Liu G (2015) Cytotoxicity and Genotoxicity in Human Embryonic Kidney Cells Exposed to Surface Modify Chitosan Nanoparticles Loaded with Curcumin. AAPS PharmSciTech. doi:10.1208/s12249-015-0471-1.

186. Van Ingen J, et al. (2011) Why do we use $600 \mathrm{mg}$ of rifampicin in tuberculosis treatment? Clin Infect Dis 52(9). doi:10.1093/cid/cir184.

187. Peterson KR, Cottam MA, Kennedy AJ, Hasty AH (2018) MacrophageTargeted Therapeutics for Metabolic Disease. Cell Press 39(6):536-546.

188. Costa A, Sarmento B, Seabra V (2018) Mannose-functionalized solid lipid nanoparticles are e ff ective in targeting alveolar macrophages. Eur J Pharm Sci 114(December 2017):103-113.

189. Maretti E, et al. (2017) Surface engineering of Solid Lipid Nanoparticle assemblies by methyl a - D -mannopyranoside for the active targeting to macrophages in anti-tuberculosis inhalation therapy. Int J Pharm 528(12):440-451.

190. Zou L, et al. (2016) Targeted delivery of nano-PTX to the brain tumorassociated macrophages. Oncotarget:1-15.

191. Binnemars-postma K, Storm G, Prakash J (2017) Nanomedicine Strategies to Target Tumor-Associated Macrophages. Int J Mol Sci 18(979):1-27. 
192. Mubin N, Umar MS, Zubair S, Owais M (2018) Selective Targeting of Functionalized Mycobacterium tuberculosis Protein Loaded Chitosan Nanoparticle to Macrophages : Correlation With Activation of Immune System. Front Microbiol 9(November):1-15.

193. Lewis RN, McElhaney RN (2000) Calorimetric and spectroscopic studies of the thermotropic phase behavior of lipid bilayer model membranes composed of a homologous series of linear saturated phosphatidylserines. Biophys $J$ 79(4):2043-2055.

194. Segawa K, Nagata S (2015) An Apoptotic "Eat Me" Signal: Phosphatidylserine Exposure. Trends Cell Biol 25(11):639-650.

195. Badalà F, Nouri-mahdavi K, Raoof DA (2008) Apoptotic cell clearance: basic biology and therapeutic potential. Nat Rev Immunol 144(5):724-732.

196. Manrique-moreno M, Heinbockel L, Suwalsky M, Garidel P, Brandenburg K (2016) Biochimica et Biophysica Acta Biophysical study of the non-steroidal anti-in fl ammatory drugs ( NSAID ) ibuprofen, naproxen and diclofenac with phosphatidylserine bilayer membranes. BBA - Biomembr 1858(9):2123-2131.

197. Joshi N, Shanmugam T, Kaviratna A, Banerjee R (2011) Proapoptotic lipid nanovesicles: Synergism with paclitaxel in human lung adenocarcinoma A549 cells. J Control Release 156(3):413-420.

198. Hosseini H, et al. (2015) Phosphatidylserine liposomes mimic apoptotic cells to attenuate atherosclerosis by expanding polyreactive IgM producing B1a lymphocytes. Cardiovasc Res 106(3):443-452.

199. Kunwar A, et al. (2008) Quantitative cellular uptake, localization and 
cytotoxicity of curcumin in normal and tumor cells. Biochim Biophys Acta Gen Subj 1780(4):673-679.

200. Cong Y, Wang L, Konrad A, Schoeb T, Elson CO (2009) Curcumin induces the tolerogenic dendritic cell that promotes differentiation of intestine-protective regulatory T cells. Eur J Immunol 39(11):3134-3146.

201. Chanburee S, Tiyaboonchai W (2017) Enhanced intestinal absorption of curcumin in Caco-2 cell monolayer using mucoadhesive nanostructured lipid carriers. J Biomed Mater Res B Appl Biomater:1-8.

202. Tima S, Anuchapreeda S, Ampasavate C, Berkland C, Okonogi S (2017) Stable curcumin-loaded polymeric micellar formulation for enhancing cellular uptake and cytotoxicity to FLT3 overexpressing EoL-1 leukemic cells. Eur J Pharm Biopharm 114:57-68.

203. Beloqui A, et al. (2014) PH-sensitive nanoparticles for colonic delivery of curcumin in inflammatory bowel disease. Int J Pharm 473(1-2):203-212.

204. Malekar SA, et al. (2015) Radio Frequency-Activated Nanoliposomes for Controlled Combination Drug Delivery. AAPS PharmSciTech 16(6):1335-43.

205. Malekar SA, Sarode AL, Bach AC, Worthen DR (2016) The Localization of Phenolic Compounds in Liposomal Bilayers and Their Effects on Surface Characteristics and Colloidal Stability. AAPS PharmSciTech 17(6):in press.

206. Procházková L, Rodríguez-Muñoz Y, Procházka J, Wanner J (2014) Simple spectrophotometric method for determination of polyvinylalcohol in different types of wastewater. Int J Environ Anal Chem 94(4):399-410.

207. Charles J, Stewart M (1980) Colorimetric Determination of Phospholipids with 
Ammonium Ferrothiocyanate. Anal Biochem 14(104):10-14.

208. Coleman JW (2001) Nitric Oxide in Immunity and Inflammation. Int Immunopharmacol 1:1397-1406.

209. Jensen DMK, et al. (2010) Spray drying of siRNA-containing PLGA nanoparticles intended for inhalation. J Control Release 142(1):138-145.

210. Barroso RP, Basso LGM, Costa-filho AJ (2015) Interactions of the antimalarial amodiaquine with lipid model membranes. Chem Phys Lipids 186:68-78.

211. Fang RH, Aryal S, Hu CMJ, Zhang L (2010) Quick synthesis of lipid-polymer hybrid nanoparticles with low polydispersity using a single-step sonication method. Langmuir 26(22):16958-16962.

212. Maretti E, et al. (2014) Inhaled Solid Lipid Microparticles to target alveolar macrophages for tuberculosis. Int J Pharm 462(1-2):74-82.

213. Jensen DK, et al. (2012) Design of an inhalable dry powder formulation of DOTAP-modified PLGA nanoparticles loaded with siRNA. J Control Release 157(1):141-148.

214. Meenach SA, et al. (2012) Synthesis, optimization, and characterization of camptothecin-loaded acetalated dextran porous microparticles for pulmonary delivery. Mol Pharm 9(2):290-298.

215. Kim I, et al. (2012) Biomaterials Doxorubicin-loaded highly porous large PLGA microparticles as a sustained- release inhalation system for the treatment of metastatic lung cancer. Biomaterials 33(22):5574-5583.

216. Azarmi S, et al. (2006) Formulation and cytotoxicity of doxorubicin nanoparticles carried by dry powder aerosol particles. Int J Pharm 319(1- 
2):155-161.

217. Sosnik A, Seremeta KP (2015) Advantages and challenges of the spray-drying technology for the production of pure drug particles and drug-loaded polymeric carriers. Adv Colloid Interface Sci 223:40-54.

218. Salama RO, et al. (2009) Preparation and evaluation of controlled release microparticles for respiratory protein therapy. J Pharm Sci 98(8):2709-2717.

219. Liang W, et al. (2015) Inhalable Dry Powder Formulations of siRNA and pHResponsive Peptides with Antiviral Activity Against H1N1 Influenza Virus. Mol Pharm 12(3):910-921.

220. Ong W, et al. (2016) Sustained Pulmonary Delivery of a Water-Soluble Antibiotic Without Encapsulating Carriers. Pharm Res 33(3):563-572.

221. Kumar A, Deepak, Sharma S, Srivastava A, Kumar R (2017) Synthesis of xanthan gum graft copolymer and its application for controlled release of highly water soluble Levofloxacin drug in aqueous medium. Carbohydr Polym 171:211-219.

222. Broaders KE, Cohen J a, Beaudette TT, Bachelder EM, Fréchet JMJ (2009) Acetalated dextran is a chemically and biologically tunable material for particulate immunotherapy. Proc Natl Acad Sci U S A 106(14):5497-502.

223. Wang Z, Meenach SA (2017) Optimization of Acetalated Dextran-Based Nanocomposite Microparticles for Deep Lung Delivery of Therapeutics via Spray-Drying. J Pharm Sci 106(12):3539-3547.

224. Finlay WH The ARLA Respiratory Deposition Calculator.

225. Sarode AL, Sandhu H, Shah N, Malick W, Zia H (2013) Hot Melt Extrusion for 
Amorphous Solid Dispersions : Temperature and Moisture Activated Drug Polymer Interactions for Enhanced Stability.

226. Vichai V, Kirtikara K (2006) Sulforhodamine B colorimetric assay for cytotoxicity screening. Nat Protoc 1(3):1112-1116.

227. Rodrigues de Azevedo C, et al. (2017) Modeling of the burst release from PLGA micro- and nanoparticles as function of physicochemical parameters and formulation characteristics. Int J Pharm 532(1):229-240.

228. Siepmann J, Siepmann F (2008) Mathematical modeling of drug delivery. Int J Pharm 364(2):328-343.

229. Singhvi G, Singh M (2011) Review : in-Vitro Drug Release Characterization Models. Int J Pharm Stud Res II(I):77-84.

230. Ungaro F, et al. (2012) Dry powders based on PLGA nanoparticles for pulmonary delivery of antibiotics: Modulation of encapsulation efficiency, release rate and lung deposition pattern by hydrophilic polymers. J Control Release 157(1):149-159.

231. Chvatal A, Farkas Á, Balásházy I, Szabó-Révész P, Ambrus R (2017) Aerodynamic properties and in silico deposition of meloxicam potassium incorporated in a carrier-free DPI pulmonary system. Int J Pharm 520(1-2):7078.

232. Salama RO, Traini D, Chan HK, Young PM (2008) Preparation and characterisation of controlled release co-spray dried drug-polymer microparticles for inhalation 2: Evaluation of in vitro release profiling methodologies for controlled release respiratory aerosols. Eur J Pharm 
Biopharm 70(1):145-152.

233. Thakral S, Terban MW, Thakral NK, Suryanarayanan R (2016) Recent advances in the characterization of amorphous pharmaceuticals by X-ray diffractometry. Adv Drug Deliv Rev 100:183-193.

234. Gallagher KM, Corrigan OI (2000) Mechanistic aspects of the release of levamisole hydrochloride from biodegradable polymers. J Control Release 69(2):261-272.

235. Corrigan OI, Li X (2009) Quantifying drug release from PLGA nanoparticulates. Eur J Pharm Sci 37(3-4):477-485.

236. Peppas NA, Sahlin JJ (1989) A simple equation for the description of solute release. III. Coupling of diffusion and relaxation. International Journal of Pharmaceutics, 1989. 57(2): p. 169-172. Int J Pharm 57(2):169-172.

237. Unagolla JM, Jayasuriya AC (2018) Drug transport mechanisms and in vitro release kinetics of vancomycin encapsulated chitosan-alginate polyelectrolyte microparticles as a controlled drug delivery system. Eur J Pharm Sci 114(December 2017):199-209.

238. Shatrohan Lal RK (2014) Synthesis of Organic Nanoparticles and their Applications in Drug Delivery and Food Nanotechnology: A Review. $J$ Nanomater Mol Nanotechnol 03(04). doi:10.4172/2324-8777.1000150.

239. Zhang L, et al. (2008) Self-assembled lipid-polymer hybrid nanoparticles: A robust drug delivery platform. ACS Nano 2(8):1696-1702.

240. Pal SL, Jana U, Manna PK, Mohanta GP, Manavalan R (2011) Nanoparticle: An overview of preparation and characterization. J Appl Pharm Sci 01(06):228- 
234.

241. Dalwadi G, Sunderland VB (2007) Purification of PEGylated nanoparticles using tangential flow filtration (TFF). Drug Dev Ind Pharm 33(9):1030-1039.

242. Dalwadi G, Sunderland B (2008) Comparison and validation of drug loading parameters of PEGylated nanoparticles purified by a diafiltration centrifugal device and tangential flow filtration. Drug Dev Ind Pharm 34(12):1331-1342.

243. Dizon-Maspat J, Bourret J, D’Agostini A, Li F (2012) Single pass tangential flow filtration to debottleneck downstream processing for therapeutic antibody production. Biotechnol Bioeng 109(4):962-970.

244. Gagnon P (2012) Technology trends in antibody purification. J Chromatogr A $1221: 57-70$.

245. Allémann E, Leroux J, Gurny R, E D (1993) In vitro extended-release properties of drug-loaded poly(DL-lactic acid) nanoparticles produced by a salting-out procedure. Pharm Res 12(10):1732-7.

246. Dimov N, Kastner E, Hussain M, Perrie Y, Szita N (2017) Formation and purification of tailored liposomes for drug delivery using a module-based micro continuous-flow system. Sci Rep 7(1):1-13.

247. Dorney KM, et al. (2014) Tangential flow filtration of colloidal silver nanoparticles: A “green” laboratory experiment for chemistry and engineering students. J Chem Educ 91(7):1044-1049.

248. Worsham RD, Thomas V, Farid SS (2019) Potential of Continous Manufacturing for Liposomal Drug Products. Biotechnol J 16:1-8.

249. Bernabeu E, Cagel M, Lagomarsino E, Moretton M, Chiappetta DA (2017) 
Paclitaxel: What has been done and the challenges remain ahead. Int J Pharm $526(1-2): 474-495$.

250. Troiano G, Nolan J, Parsons D, Van Geen Hoven C, Zale S (2016) A Quality by Design Approach to Developing and Manufacturing Polymeric Nanoparticle Drug Products. AAPS J 18(6):1354-1365.

251. Yerlikaya F, et al. (2013) Development and evaluation of paclitaxel nanoparticles using a quality-by-design approach. J Pharm Sci 102(10):37483761.

252. Zong Y, Wu J, Shen K (2017) Nanoparticle albumin-bound paclitaxel as neoadjuvant chemotherapy of breast cancer: a systematic review and metaanalysis. Oncotarget 8(10):17360-17372.

253. Tomé-Carneiro J, et al. (2013) Resveratrol and Clinical Trials: The Crossroad from In Vitro Studies to Human Evidence. Curr Pharm Des 19(34):6064-6093.

254. Dheer D, Jyoti, Gupta PN, Shankar R (2018) Tacrolimus: An updated review on delivering strategies for multifarious diseases. Eur J Pharm Sci 114(October 2017):217-227.

255. Spiekerkoetter E, et al. (2015) Low-Dose FK506 (Tacrolimus) in End-Stage Pulmonary Arterial Hypertension. Am J Respir Crit Care Med 192(2):254-257.

256. Chen Y-L, Hirabayashi H, Akhtar S, Pelzer M, Kobayashi M (2006) Simultaneous determination of three isomeric metabolites of tacrolimus (FK506) in human whole blood and plasma using high performance liquid chromatography-tandem mass spectrometry. J Chromatogr B 830(2):330-341.

257. Hu J, et al. (2015) Novel Targets of Drug Treatment for Pulmonary 
Hypertension. Am J Cardiovasc Drugs 15(4):225-234.

258. A AMA, et al. (2016) Activation of Anoctamin-1 Limits Pulmonary Endothelial Cell proliferation via p38- MAPK-dependent Apoptosis. AJRCMB:1-53.

259. Montani D, et al. (2014) Targeted therapies in pulmonary arterial hypertension. Pharmacol Ther 141(2):172-91.

260. Lambert M, et al. (2018) Ion Channels in Pulmonary Hypertension : A Therapeutic Interest? Int J Mol Sci 19(3162):1-49.

261. Evans JDW, et al. (2016) BMPR2 mutations and survival in pulmonary arterial hypertension : an individual participant data meta-analysis. Lancet Respir Med $4: 129-137$.

262. West J, Austin E, Fessel JP, Loyd J, Hamid R (2014) Rescuing the BMPR2 signaling axis in pulmonary arterial hypertension. Drug Discov Today 19(8):1241-1245.

263. Spiekerkoetter E, et al. (2013) FK506 activates BMPR2, rescues endothelial dysfunction, and reverses pulmonary hypertension. J Clin Invest 123(8):36003613.

264. Qi J, Wang Y, Liu Y, Zhang F (2014) Development and validation of HTS assay for screening the calcium-activated chloride channel modulators in TMEM16A stably expressed CHO cells. Anal Bioanal Chem 406:1713-1721.

265. Ji Q, Wang X, An H (2018) Recent advances in TMEM16A : Structure, function, and disease. J Cell Physiol (November):1-18.

266. Leblanc N, et al. (2015) Molecular and functional significance of Ca $2+-$ activated $\mathrm{Cl}$ - channels in pulmonary arterial smooth muscle. Pulm Circ 
$5(2): 244-268$.

267. Forrest AS, et al. (2012) Increased TMEM16A-encoded calcium-activated chloride channel activity is associated with pulmonary hypertension. Am J Physiol Cell Physiol 303:1229-1243.

268. Forrest AS, et al. (2012) Increased TMEM16A-encoded calcium-activated chloride channel activity is associated with pulmonary hypertension. Am J Physiol Cell Physiol 303(12):C1229-43.

269. Jiang Y, Yu B, Yang H, Ma T (2016) Shikonin Inhibits Intestinal CalciumActivated Chloride Channels and Prevents Rotaviral Diarrhea. Front Pharmacol 7(August):1-9.

270. Hübner CA, Schroeder BC, Jena FU, Schroeder BC (2015) Regulation of vascular tone and arterial blood pressure : role of chloride transport in vascular smooth muscle. Pflugers Arch-Eur J Physiol 467:605-614.

271. Oh U, Jung J (2016) Cellular functions of TMEM16 / anoctamin. Pflugers Arch-Eur J Physiol 468:443-453.

272. Sun H, Xia Y, Paudel O, Yang X-R, Sham JSK (2012) Chronic hypoxiainduced upregulation of $\mathrm{Ca} 2+$-activated $\mathrm{Cl}-$ channel in pulmonary arterial myocytes: a mechanism contributing to enhanced vasoreactivity. J Physiol 590(15):3507-3521.

273. Namkung W, Yao Z, Finkbeiner WE, Verkman AS (2011) Small-molecule activators of TMEM16A, a calcium-activated chloride channel, stimulate epithelial chloride secretion and intestinal contraction. FASEB J 25(11):40484062. 
274. Liu S, et al. (2016) Eact, a small molecule activator of TMEM16A, activates TRPV1 and elicits pain- and itch-related behaviours. Br J Pharmacol 173(7):1208-1218.

275. Burris SK, Wang Q, Bulley S, Neeb ZP (2015) 9-Phenanthrol inhibits recombinant and arterial myocyte TMEM16A channels. Br J Pharmacol 172:2459-2468.

276. Pilcer G, Vanderbist F, Amighi K (2009) Preparation and characterization of spray-dried tobramycin powders containing nanoparticles for pulmonary delivery. Int J Pharm 365(1-2):162-169.

277. Meenach SA, Anderson KW, Zach Hilt J, McGarry RC, Mansour HM (2013) Characterization and aerosol dispersion performance of advanced spray-dried chemotherapeutic PEGylated phospholipid particles for dry powder inhalation delivery in lung cancer. Eur J Pharm Sci 49(4):699-711.

278. Sarode AL, Sandhu H, Shah N, Malick W, Zia H (2013) European Journal of Pharmaceutical Sciences Hot melt extrusion ( HME ) for amorphous solid dispersions : Predictive tools for processing and impact of drug - polymer interactions on supersaturation. Eur J Pharm Sci 48(3):371-384.

279. Li X, Vogt FG, Hayes D, Mansour HM (2014) Design, characterization, and aerosol dispersion performance modeling of advanced co-spray dried antibiotics with mannitol as respirable microparticles/nanoparticles for targeted pulmonary delivery as dry powder inhalers. J Pharm Sci 103(9):2937-2949.

280. Walters RH, Bhatnagar B, Tchessalov S, Izutsu K (2014) Next Generation Drying Technologies for Pharmaceutical Applications. J Pharm Sci 
103(9):2673-2695.

281. Hancock BC, Shamblin SL, Zografi G (1995) Molecular Mobility of Amorphous Pharmaceutical Solids Below Their Glass Transition Temperatures. Pharm Res An Off J Am Assoc Pharm Sci 12(6):799-806.

282. Tian Y, Jones DS, Andrews GP (2015) An investigation into the role of polymeric carriers on crystal growth within amorphous solid dispersion systems. Mol Pharm 12(4):1180-1192.

283. Edueng K, Mahlin D, Larsson P, Bergström CAS (2017) Mechanism-based selection of stabilization strategy for amorphous formulations: Insights into crystallization pathways. J Control Release 256(January):193-202.

284. Andronis V, Yoshioka M, Zografi G (1997) Effects of sorbed water on the crystallization of indomethacin from the amorphous state. J Pharm Sci 86(3):346-357.

285. Park CW, et al. (2013) Advanced spray-dried design, physicochemical characterization, and aerosol dispersion performance of vancomycin and clarithromycin multifunctional controlled release particles for targeted respiratory delivery as dry powder inhalation aerosols. Int J Pharm 455(12):374-392.

286. Costa, P., \& Lobo JMS (2001) Modelling and Comparison of Dissolution Profiles. Eur J Pharm Sci 13:123-133. 